June 2009

\title{
Local Grand Unification in the Heterotic Landscape
}

\author{
Jonas Schmidt \\ Deutsches Elektronen-Synchrotron DESY, Notkestrasse 85, 22607 Hamburg, Germany
}

\begin{abstract}
We consider the possibility that the unification of the electroweak interactions and the strong force arises from string theory, at energies significantly lower than the string scale. As a tool, an effective grand unified field theory in six dimensions is derived from an anisotropic orbifold compactification of the heterotic string. It is explicitly shown that all anomalies cancel in the model, though anomalous Abelian gauge symmetries are present locally at the boundary singularities. In the supersymmetric vacuum additional interactions arise from higher-dimensional operators. We develop methods that relate the couplings of the effective theory to the location of the vacuum, and find that unbroken discrete symmetries play an important role for the phenomenology of orbifold models. An efficient algorithm for the calculation of the superpotential to arbitrary order is developed, based on symmetry arguments. We furthermore present a correspondence between bulk fields of the orbifold model in six dimensions, and the moduli fields that arise from compactifying four internal dimensions on a manifold with non-trivial gauge background.
\end{abstract}




\section{Contents}

1 Introduction $\quad 4$

2 The heterotic string on orbifolds $\quad 7$

2.1 The world sheet action . . . . . . . . . . . . . . . . . 8

2.2 Classical strings on orbifolds . . . . . . . . . . . . . . . . . 10

2.2.1 The orbifold geometry . . . . . . . . . . . . . . . 10

2.2.2 Orbifold boundary conditions . . . . . . . . . . . . . . . 12

2.2 .3 Classical solutions . . . . . . . . . . . . . . . . . . . 15

2.3 Quantization and the low-energy spectrum . . . . . . . . . . . . 16

2.3.1 The Hilbert space . . . . . . . . . . . . . . . . . . . . . 16

2.3 .2 Orbifold compatibility . . . . . . . . . . . . . 17

2.3.3 Conditions for massless states . . . . . . . . . . . . . . . 22

2.3.4 The generic spectrum . . . . . . . . . . . . . . . 22

2.4 Interactions . . . . . . . . . . . . . . . . . . . . . . . . . . . . . . .

2.4.1 Gauge invariance and discrete $R$-symmetry . . . . . . . . . . . 25

2.4 .2 Space group selection rule . . . . . . . . . . . . . . . . 26

3 A local GUT in six dimensions $\quad 27$

3.1 The $\mathbb{Z}_{6-\text { II }}$ orbifold . . . . . . . . . . . . . . . . . . . . 27

3.1.1 The gauge embedding . . . . . . . . . . . . . . 32

3.1.2 Space group selection rules for interactions . . . . . . . . . . . . . 32

3.2 The effective orbifold GUT . . . . . . . . . . . . . . . . . . . . . . . . . . . . . . . . 34

3.2.1 Anisotropic limits of orbifolds . . . . . . . . . . . . . . . . 34

3.2 .2 Geometry of the effective GUT . . . . . . . . . . . . . 37

3.2.3 Generic projection conditions . . . . . . . . . . . . . . 38

3.2 .4 The gravity sector . . . . . . . . . . . . . . . . . . . . . . . . . . . . . . . . . .

3.2 .5 Gauge vectors . . . . . . . . . . . . . . . . . . . . . . 42

3.2 .6 Matter states . . . . . . . . . . . . . . . . . 46

3.2.7 The effective orbifold GUT field theory . . . . . . . . . . . 53

3.3 Anomaly cancelation . . . . . . . . . . . . . . . . 56

3.3.1 The Stora-Zumino descent relations . . . . . . . . . . . . . . 58

3.3 .2 The bulk anomaly polynomial . . . . . . . . . . . . . . . 60

3.3.3 Anomaly polynomials at fixed points . . . . . . . . . . 63

3.3.4 Green-Schwarz anomaly cancelation in six dimensions . . . . . . . 65

3.3.5 Green-Schwarz anomaly cancelation on the orbifold . . . . . . . . 66

4 Vacua and phenomenology $\quad \mathbf{6 7}$

4.1 The SU(5) GUT superpotential . . . . . . . . . . . . . . . . . . . . . . . . . . . . . 68

4.2 Standard model families and a unique $\mathrm{U}(1)_{B-L} \ldots \ldots . \ldots . \ldots 71$

$4.3 \quad F$-terms and $D$-terms . . . . . . . . . . . . . . . . . . . . 73 
4.4 Decoupling of exotics . . . . . . . . . . . . . . . . . 74

4.4 A minimal vacuum . . . . . . . . . . . . . . . . . 76

4.5 Unbroken symmetries . . . . . . . . . . . . . . . . . . . . . . . . . . . . . . . . . . . . . .

4.5.1 Symmetries of the false vacuum . . . . . . . . . . . . . 78

4.5.2 Symmetries of physical vacua . . . . . . . . . . . . . . . 79

4.5.3 Symmetries and interactions . . . . . . . . . . . . . . 81

4.6 Vacuum selection . . . . . . . . . . . . . . . . . . . . . 84

4.6.1 Maximal vacua for vanishing couplings . . . . . . . . . . . . . . . 85

4.6.2 Gauge-Higgs unification . . . . . . . . . . . . . . . 86

4.6.3 Partial gauge-Higgs unification . . . . . . . . . . . . . . . 90

4.7 The all-order superpotential . . . . . . . . . . . . . . . . . . . . . . . . . . . . 94

4.7.1 The calculation of F-terms . . . . . . . . . . . . . 96

5 Connection with smooth geometries $\quad 96$

5.1 Compactification on the $K 3$ surface . . . . . . . . . . . . . . . . 97

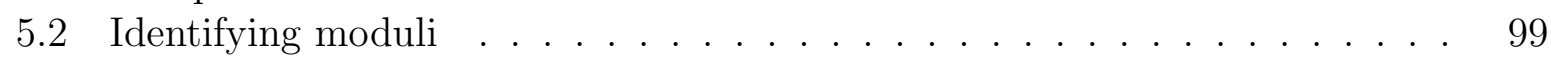

5.3 Outlook: A local GUT based on K3 . . . . . . . . . . . . . . . . . . . 100

$\begin{array}{llr}6 & \text { Conclusions } & 100\end{array}$

$\begin{array}{ll}\text { A Orbifold details } & 102\end{array}$

A.1 Solving the fixed point equation . . . . . . . . . . . . . . . . 102

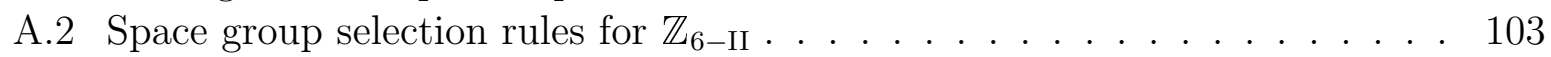

A.3 The gamma phases . . . . . . . . . . . . . . . . . . . . . . . . . . . . . . . . . . . . . . . . . . .

A.3.1 The general case . . . . . . . . . . . . . . . . . . . . . 104

A.3.2 The $\mathbb{Z}_{6-\text { II }}$ example . . . . . . . . . . . . . . . . 107

$\begin{array}{ll}\text { B Lie Group Basics } & 108\end{array}$

$\begin{array}{ll}\text { C Anomalies with Abelian factors } & 111\end{array}$

C.1 Bulk anomalies . . . . . . . . . . . . . . . . . . . . 111

C.2 Fixed point anomalies . . . . . . . . . . . . . . . 112

$\begin{array}{ll}\text { D The hidden brane } & 115\end{array}$

$\begin{array}{ll}\text { E Zero modes in four dimensions } & 118\end{array}$

$\begin{array}{llr}\text { F Discrete symmetry transformations } & 122\end{array}$

$\begin{array}{lr}\text { Bibliography } & 129\end{array}$

$\begin{array}{lr}\text { Index } & 139\end{array}$ 


\section{Introduction}

The vast majority of experimental observations at high-energy facilities in the pre-LHC era is described to very high accuracy by the standard model of particle physics [1]. The latter is a phenomenological model whose 19 parameters are deduced from the best fit to the available data. Major drawbacks of the standard model are that it fails to describe neutrino oscillations and the observed abundance of dark matter and dark energy in the universe. Further issues concern the Higgs sector. The sensitivity of the Higgs mass to the cutoff scale implies large fine-tuning and suggests the existence of beyond standard model physics, such as supersymmetry (SUSY). In addition, the triviality problem of the quartic Higgs self-interaction at infinite cutoff, i.e. the expected vanishing of the corresponding coupling constant at low energies in that limit [2,3], indicates that the standard model is not fundamental. Attempts of treating gravity and the other forces of nature on the same footing, namely as local gauge symmetries, lead to the same conclusion ${ }^{1}$. This is due to incurable ultraviolet divergencies, which are also present for supergravity (SUGRA) extensions of the standard model, i.e. for local supersymmetry.

The most popular candidate for a fundamental theory is string theory [6-9]. It is based on replacing the idea of elementary point particles by the concept of fundamental strings. Due to their one-dimensional nature, string interactions are not point-like and therefore finite by construction. Consistency arguments then imply that strings have to propagate in ten space-time dimensions. Since they include a description of gravity, the sole scale $M_{s}$ of string theory is set by the analogue of Newton's constant in ten dimensions (up to the string coupling $g_{s} \sim \exp (\phi)$, where $\phi$ denotes the dilaton). Effective theories in four dimensions are obtained by assuming that six space-like coordinates describe a compact geometry. Its size, the string coupling, and the string scale then combine to the four-dimensional Planck scale $M_{P} \sim 10^{19} \mathrm{GeV}$.

As yet it remains an open question if string theory describes nature. It is known to have ten to the hundreds different vacuum solutions, related to assumptions about the geometry of the predicted extra dimensions, but it is not known whether the standard model is accommodated in any of them. However, explicit constructions have shown that one can get indeed very close to the standard model and study quasi-realistic vacua. Examples include heterotic string compactifications on smooth [10] or singular [11-17] geometries (see [18] for recent reviews), intersecting D-branes and orientifold models (cf. [19-21] and references therein), free fermionic models [22], and F-theory constructions [23]. All models have in common that they do not fully match low-energy phenomenology, but almost, and it does not seem impossible to discover the missing link.

A promising strategy for identifying a string theory vacuum whose low-energy effective field theory coincides with the standard model may be to require that the electroweak and the strong forces unify at an intermediate scale. The existence of a grand unified theory (GUT) [1] in nature is an assumption, backed by the group theoretical simplicity of

\footnotetext{
${ }^{1}$ Asymptotic safety scenarios of gravity [4] and the Higgs/Yukawa sector [5] provide an alternative viewpoint, giving up the premise of perturbativity.
} 
the early Georgi-Glashow SU(5) [24] and SO(10) [25] GUT proposals, and a quantitative argument from the renormalization group running of the coupling constants. For the minimal supersymmetric standard model (MSSM), with SUSY partner masses of order of the electroweak scale $M_{\mathrm{EW}} \sim 3 \cdot 10^{2} \mathrm{GeV}$, unification appears at $M_{\mathrm{GUT}} \sim 3 \cdot 10^{16}$, up to $4 \%$ threshold corrections (cf. [26]). If a GUT is realized in nature, this result suggests that also supersymmetry is likely to exist, with a soft mass scale related to the electroweak scale. For GUTs in accord with string theory, the phenomenological value of the gauge coupling at the GUT scale $\alpha_{\mathrm{GUT}} \sim 1 / 25$ implies a further relation between the string scale $M_{s}$, the volume of the compact internal dimensions, and the string coupling [27].

The simplest possibility for constructing supersymmetric grand unified theories in higher dimensions from string theory [28] may be the compactification of the heterotic string [29] on orbifolds [30]. One version of heterotic string theory is equipped with the gauge group $\mathrm{E}_{8} \times \mathrm{E}_{8}$, where the exceptional Lie group $\mathrm{E}_{8}$ inherits standard GUT groups, and may therefore provide a promising framework.

Orbifolds are simple examples for compact internal spaces. They are flat except for singular boundary points, called 'fixed points', and thus technically easy to handle. In field theoretical model building, orbifolds became a popular approach for higher-dimensional GUTs more than five years ago [31-35] (see [36,37] for recent work on 5d orbifolds). Standard problems of four-dimensional GUTs, like the breaking of the larger gauge group to the standard model or the presence of exotics in the larger Higgs representations, have simple solutions in orbifold models in terms of the boundary conditions of the bulk fields. Furthermore, orbifold GUTs lead to the idea of 'local grand unification' at the fixed points [38]. The fact that matter and Higgs fields may have different localization properties on the compact space can lead to interesting phenomenology, like the outcome of a heavy top-quark, or, more generally, non-trivial predictions concerning flavor physics.

The phenomenological success of field theoretical orbifold SUSY GUTs in higher dimensions led to a renewed interest in heterotic orbifold models, twenty years after the first proposals of three-family orbifold models [39-41]. The model [13] was found by requiring local $\mathrm{SO}(10)$ unification, and this approach turned out to be very fruitful. For the first time it was possible to decouple all exotics in a consistent string compactification, with quasirealistic Yukawa couplings and phenomenology. Once this model was found, a systematic scan of the 'mini-landscape' of similar heterotic orbifold models could be carried out, and approximately 300 standard model-like string compactifications were identified [14].

The motivation behind the model [13], however, was the idea to realize an orbifold GUT in six dimensions, similar to the field theoretical model [34], in string theory. The corresponding anisotropic limit [42] was worked out in full detail in [16], including the explicit cancelation of all anomalies. The construction relies on the assumption that some of the compact internal dimensions are considerably larger than others, reflecting the gap between the GUT and the string scale. Note that anisotropic orbifolds seem to be preferred by the perturbative heterotic string [43] (for work on precision gauge coupling unification in similar models see [44]). An explicit mechanism to stabilize the large dimensions at the GUT scale was addressed recently in $[37,45]$. The effective six-dimensional orbifold GUT [16] has local $\mathrm{SU}(5) \times \mathrm{U}(1)_{X}$ unification, a unique $\mathrm{U}(1)_{B-L}$, matter parity, a heavy 
top-quark, and a simple decoupling mechanism for most of the exotics [16,17]. This model will be reviewed and studied in detail in this paper, bearing in mind that it can be seen as a representative of a full class of quasi-realistic string compactifications [14].

Most work in the literature has been concerned with compactifications directly to four dimensions. One typically obtains a large number of fields in heterotic orbifold models, and it is not straightforward to distinguish matter fields, Higgs fields and exotics. In the favored models exotic fields are vector-like, and can therefore be decoupled by the generation of mass terms, after the transition to the supersymmetric vacuum. The latter generically corresponds to non-trivial configurations in field space. This is the consequence of a tachyonic direction that is sourced by a loop-induced Fayet-Iliopoulos term (cf. [46,47]). The requirement of non-vanishing vacuum expectation values (vevs) for some of the fields is an important point for orbifold model building, since it leads to the simultaneous generation of infinitely many interaction terms. The strength of the effective coupling then scales with the number of involved vevs, analogue to the Froggatt-Nielsen mechanism [48]. The generated interactions may be favored, like Yukawa couplings, or disfavored, like proton decay operators or large $\mu$-terms. The phenomenology is therefore largely determined by the set of singlets that acquire non-zero vacuum expectation values.

Up to now, finding quasi-realistic vacua in heterotic orbifold models seems to be mainly an issue of computing power. In practice, one truncates the superpotential at a chosen finite order in the singlet vevs (typically this order is 6 or $8[13,14,49]$ ), and scans for vacuum configurations for which the $F$-term and the $D$-term equations can be fulfilled, with satisfactory phenomenology. However, one may ask if the search for vacua with phenomenologically preferred properties could be improved by detailed studies of the connection between the singlets which contribute to the vacuum, and the resulting effective superpotential. We shall address this problem in this work by considering symmetry arguments that may help to identify interesting candidates for physical vacua. This extends the results of [17], where the importance of unbroken discrete symmetries was noted first. Since the symmetries in non-trivial vacuum configurations refer to the full (perturbative) superpotential, they can be used to forbid disfavored terms to arbitrary order in the singlet vevs $^{2}$. The detailed discussion of this method and its application to the effective orbifold GUT model in six dimensions is the main purpose of this work.

The transition to the supersymmetric vacuum is related to a consistency issue which generically arises in orbifold compactifications. The back-reaction of the vacuum expectation values of localized twisted states on the geometry corresponds to 'blowing-up' the orbifold singularities (cf. [40,51-55] and [56] for a recent analysis of the $\mathbb{Z}_{6-\mathrm{II}}$ case). The general observation is that the resulting smooth compactification has less zero modes and reduced symmetries. The claim that one may ignore this back-reaction in the vicinity of the orbifold point, i.e. that the orbifold description remains a good approximation nearby the origin in field space, still lacks a rigorous proof. However, it seems plausible from the observation that orbifolds correspond to specific points of the moduli spaces associated

\footnotetext{
${ }^{2}$ Alternatively, a sufficient suppression may be obtained if the symmetries are broken at high order in the singlets, cf. [50].
} 
with manifolds. The matching of spectra of the orbifold and a smooth background geometry may then give insights on the role of the orbifold states and thus lead to a better understanding of orbifold vacua. Although this work is mostly concerned with orbifolds, we also comment briefly on the interpretation of the bulk states in six dimensions as zero modes of a smooth compactification.

This paper is organized as follows. Chapter 2 introduces the heterotic string and reviews its compactification on an orbifold, clarifying the role of lattice translations for the orbifold projection conditions in Section 2.3.2. The spectrum of the effective orbifold GUT model in six dimensions is calculated in Chapter 3, corresponding to an anisotropic limit of the compactification. Section 3.3 explicitly confirms that all appearing anomalies are canceled. In Chapter 4 , we discuss the model in a non-trivial vacuum configuration. The superpotential of standard SU(5) GUTs is reviewed in Section 4.1, before we turn to the local GUTs with that gauge symmetry at the fixed points in the extra dimensions. Section 4.2 identifies unique Abelian symmetries $\mathrm{U}(1)_{X}$ and $\mathrm{U}(1)_{B-L}$ and the standard model families. A minimal vacuum that preserves matter parity and implies a universal decoupling of many exotics is defined in Section 4.4. The symmetries of general vacua are analyzed in Section 4.5, furthermore it is shown for the above minimal vacuum symmetries and vanishing couplings are in direct correlation. Section 4.6 describes an algorithm which allows one to enlarge the vacuum without generating disfavored couplings, and two examples are discussed. Section 4.7 then provides a method which can be used for the calculation of superpotential interactions to arbitrary order in a given vacuum, with improved efficiency compared to order-by-order scans. An outlook on work in progress concerning a connection of the model with smooth geometries is given in Chapter 5, before we conclude in Chapter 6. Some technical details of orbifold compactifications are studied in Appendix A, basic facts about Lie groups and Lie algebras are collected in Appendix B. Appendix C gives details of the calculation of the reducible anomalies of the model. The states at the hidden fixed points and the four-dimensional zero modes are listed in appendices D and E, respectively. Finally, Appendix F summarizes the transformation behavior of all fields at the GUT fixed points with respect to the unbroken symmetries of the three studied vacua. From this information it can be inferred whether a particular coupling may be present in the vacuum, or is forbidden to arbitrary order in the singlets.

\section{The heterotic string on orbifolds}

This chapter briefly introduces the heterotic string [29] and describes its compactification on orbifolds [30]. The results and ideas presented here are neither new nor do they originate from the author, except for the ansatz for the superposition of physical states in Appendix A.3. Instead they can be found in much more detail in textbooks, e.g. [7-9], or reviews $[18,57]$. Here we widely follow the conventions of $[13,58]$. The main purpose of this section is to fix the notation and to present the foundations of the model which will be discussed in detail in this work. 


\subsection{The world sheet action}

The heterotic string can be understood as a field theory on a two-dimensional surface, called the 'string world-sheet', which is parametrized by one time-like and one space-like direction $\tau$ and $\sigma$, respectively. The theory can be formulated in terms of 26 bosonic fields $X^{\mu}, X^{I}, \mu=0, \ldots, 9, I=1, \ldots, 16$, and 10 fermionic fields $\psi^{\mu}$, which play the role of coordinates of a target space. Up to specification of the metrics on the world-sheet and the target space, the action reads ${ }^{3}$

$$
S=-\frac{M_{s}^{2}}{2 \pi} \int \mathrm{d}^{2} \sigma\left[\partial_{\alpha} X_{\mu} \partial^{\alpha} X^{\mu}+4 i \psi^{\mu}\left(\partial_{\tau}-\partial_{\sigma}\right) \psi_{\mu}+\sum_{I=1}^{16} \partial_{\alpha} X^{I} \partial^{\alpha} X^{I}\right] .
$$

Here $M_{s}$ is the string scale. On-shell, the string fields appearing in this action can be written in terms of functions of the combinations $\sigma_{ \pm}=\tau \pm \sigma$,

$$
X^{\mu}(\tau, \sigma)=X_{L}^{\mu}\left(\sigma_{+}\right)+X_{R}^{\mu}\left(\sigma_{-}\right), \quad \psi^{\mu}(\tau, \sigma)=\psi_{R}^{\mu}\left(\sigma_{-}\right), \quad X^{I}(\tau, \sigma)=X_{L}^{I}\left(\sigma_{+}\right),
$$

where subscripts $L, R$ were introduced for left- and right-moving fields , respectively. Due to the asymmetry between left-movers and right-movers only closed heterotic strings exist, which implies specific boundary conditions at $\sigma$ and $\sigma+\pi$ in our conventions.

The target space contains the physical four-dimensional Minkowski space $M_{4}$. A consistent quantum theory can be formulated if the latter is embedded into its ten-dimensional analogue $M_{10}$, labeled by the index $\mu$. Additionally, the index $I$ labels coordinates of a 16-dimensional internal compact space, which has to coincide with the root lattice of either $E_{8} \times E_{8}$ or $\operatorname{Spin}(32) / \mathbb{Z}_{2}$, in order to obtain a consistent (quantum) theory in ten dimensions. The corresponding fields $X_{L}^{I}$ are then interpreted as gauge fields. Throughout the whole work we shall consider the case of a gauge group

$$
G_{10}=E_{8} \times E_{8} .
$$

The field content of heterotic string theory is sketched in Figure 1. The presence of the labels $\mu$ and $I$ is a consequence of the heterotic nature of the theory: While $X_{R}^{\mu}$ and $\psi_{R}^{\mu}$ form a superstring in ten dimensions, $X_{L}^{\mu}, X_{L}^{I}$ are fields of a bosonic string in 26 dimensions. Thus supersymmetry charges are carried by right-moving fields, gauge charges by leftmovers. For quantization the product of left- and right-movers will be relevant and this information is combined, yielding states which form full multiplets of $\mathcal{N}=1$ supergravity in ten dimensions and of the gauge group (3), at each mass level.

The theory which has been described so far does not describe nature as we observe it. First, it requires ten space-time dimensions, and second, it has too much supersymmetry, namely 16 supercharges. A common approach is to replace the ten-dimensional target space by the product space of a compact six-dimensional geometry and the four-dimensional noncompact space,

$$
\mathcal{M}_{10}=\mathcal{M}_{6} \times M_{4}
$$

\footnotetext{
${ }^{3}$ Throughout this paper we follow the Einstein sum convention, if not stated otherwise.
} 


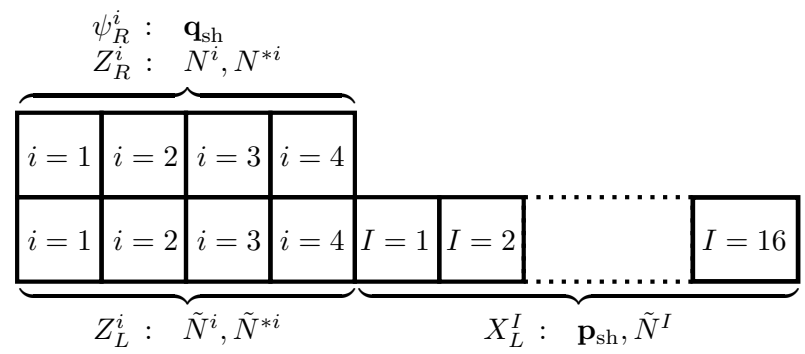

Figure 1: The field content of the heterotic string. Instead of the fields $X_{L, R}^{\mu}, \psi_{R}^{\mu}, \mu=1, \ldots, 8$, the complex coordinates $Z_{L, R}^{i}=\frac{1}{\sqrt{2}}\left(X_{L, R}^{2 i-1}+i X_{L, R}^{2 i}\right), i=1, \ldots, 4$, and likewise for $\psi_{R}^{i}$, are shown. After quantization, the fields give rise to states of a Hilbert space. $\mathbf{q}_{\mathrm{sh}}$ and $\mathbf{p}_{\mathrm{sh}}$ represent charges under the little group and the gauge group of these states, respectively, and $N^{i}, N^{* i}, \tilde{N}^{i}, \tilde{N}^{* i}, \tilde{N}^{I}$ denote oscillator numbers.

If $\mathcal{M}_{6}$ has small volume, the effective theory at low energies is four-dimensional. Furthermore, compactification on $\mathcal{M}_{6}$ can be responsible for supersymmetry and gauge symmetry breaking, possibly yielding the standard model in four dimensions. However, for non-flat manifolds $\mathcal{M}_{6}$ the quantization of the theory is in general unknown. In that situation one usually considers the ten-dimensional supergravity on $\mathcal{M}_{10}$ as an effective theory. Since in this work we are interested in compactifications on orbifolds, which are flat except for singular points at the boundary, we are in the comfortable position to directly solve the dynamics of the string fields and then quantize canonically.

The canonical momenta which follow from the action (1) are not independent. In light-cone gauge the unphysical coordinates are expressed as combinations of the time-like direction $(\mu=0)$ and one of the spatial directions (we choose $\mu=9$ ),

$$
X^{ \pm}(\tau, \sigma)=\frac{1}{\sqrt{2}}\left(X^{0}(\tau, \sigma) \pm X^{9}(\tau, \sigma)\right), \quad \psi^{ \pm}\left(\sigma_{-}\right)=\frac{1}{\sqrt{2}}\left(\psi^{0}\left(\sigma_{-}\right) \pm \psi^{9}\left(\sigma_{-}\right)\right) .
$$

The independent directions are then the transverse coordinates $\mu=1, \ldots, 8$, with equations of motion

$$
\partial_{+} \psi^{\mu}=0, \quad \partial_{+} \partial_{-} X^{\mu}=0, \quad \partial_{+} \partial_{-} X^{I}=0,
$$

where $\partial_{ \pm}=\partial / \partial \sigma_{ \pm}$and $I=1, \ldots, 16$. Solutions to these equations will then allow straightforward canonical quantization. However, they depend on boundary conditions of the fields, which eventually make the crucial difference between toroidal and orbifold compactifications. 


\subsection{Classical strings on orbifolds}

\subsubsection{The orbifold geometry}

In the following we shall consider orbifold compactifications of the type

$$
\mathcal{M}_{6}=\frac{T^{2} \times T^{2} \times T^{2}}{\mathbb{Z}_{N}},
$$

where $T^{2}$ denotes a two-dimensional torus and $N$ is an integer number ${ }^{4}$. The product form of $\mathcal{M}_{6}$ suggests to introduce three complex coordinates for the internal space ${ }^{5}$,

$$
Z^{1}=\frac{1}{\sqrt{2}}\left(X^{1}+i X^{2}\right), \quad Z^{2}=\frac{1}{\sqrt{2}}\left(X^{3}+i X^{4}\right), \quad Z^{3}=\frac{1}{\sqrt{2}}\left(X^{5}+i X^{6}\right) .
$$

Similarly, we introduce another complex coordinate for the two transversal directions in four-dimensional Minkowski space, $Z^{4}=\left(X^{7}+i X^{8}\right) / \sqrt{2}$.

\section{The space group}

The action of a generating element of the 'point group' $\mathbb{Z}_{N}$ in $(7)$ on the toroidal coordinates $Z^{i}$ can now be defined in terms of a 'twist vector' $v$, with entries of order $N$,

$$
\mathbf{v}=\left(v^{1}, v^{2}, v^{3} ; 0\right), \quad \quad N v^{j} \in \mathbb{Z}, \quad j=1,2,3 .
$$

The zero in the last entry was included for later convenience. A coordinate $Z^{i}$ then transforms as (here no summation over $i$ is implied)

$$
Z^{i} \rightarrow \vartheta_{(i)} Z^{i}, \quad \vartheta_{(i)} \equiv e^{2 \pi i v^{i}}, \quad i=1, \ldots, 4 .
$$

Thus the coordinates of the internal tori are rotated by the point group $\mathbb{Z}_{N}$, and the latter is embedded into internal Lorentz transformations. Since the on-shell degrees of freedom transform under the little group $\mathrm{SO}(8)$, we have

$$
\mathbb{Z}_{N} \subset \mathrm{SO}(8) \subset \mathrm{SO}(1,9) .
$$

As it turns out, there are additional phenomenological constraints on the twist vector, due to the requirement that the resulting low-energy spectrum should have $\mathcal{N}=1$ supersymmetry:

$$
\sum_{j} v^{j}=0, \quad v^{j} \neq 0 \bmod 1, \quad j=1,2,3 .
$$

\footnotetext{
${ }^{4}$ For $\mathbb{Z}_{N} \times \mathbb{Z}_{M}$ orbifolds see e.g. [18].

${ }^{5}$ In this section we do not explicitly show the dependence of coordinates $X^{i}$ on the world-sheet variables $(\tau, \sigma)$. All following transformation rules apply point-wise with respect to these variables.
} 
These conditions will become transparent during the discussion of the generic spectrum in Section 2.3.4.

Further isometries of the geometry (7) are lattice translations of the three tori. Each of them is defined as $T^{2}=\mathbb{C} / \pi \Lambda^{j}$ for a two-dimensional lattice $\Lambda^{j}$, with lattice vectors $\mathbf{e}_{2 j-1}, \mathbf{e}_{2 j} \in \mathbb{C}^{4}, j=1,2,3$, of the form

$$
\begin{array}{lll}
\Lambda^{1}: & \mathbf{e}_{1}=\left(e_{1}^{1}, 0,0 ; 0\right) & \mathbf{e}_{2}=\left(e_{2}^{1}, 0,0 ; 0\right) \\
\Lambda^{2}: & \mathbf{e}_{3}=\left(0, e_{3}^{2}, 0 ; 0\right) & \mathbf{e}_{4}=\left(0, e_{4}^{2}, 0 ; 0\right) \\
\Lambda^{3}: & \mathbf{e}_{5}=\left(0,0, e_{5}^{3} ; 0\right) & \mathbf{e}_{6}=\left(0,0, e_{6}^{3} ; 0\right),
\end{array}
$$

with complex entries $e_{a}^{i}$. The product of the three tori can then be understood as $\mathbb{C}^{3} / \pi \Lambda$, with $\Lambda=\sum_{j} \Lambda^{j}$. The compactness of the internal space is expressed as invariance under translations

$$
Z^{i} \rightarrow Z^{i}+\pi m_{a} e_{a}^{i}, \quad \quad m_{a} \in \mathbb{Z}, \quad i=1, \ldots, 4 .
$$

In summary the 'space group', which is the total isometry group of the space under consideration, can be written as

$$
S=\left\{\left(\theta^{k}, m_{a} \mathbf{e}_{a}\right) \mid m_{a} \in \mathbb{Z}, k=0, \ldots,(N-1)\right\},
$$

where $\theta=\operatorname{diag}\left(\vartheta_{(1)}, \vartheta_{(2)}, \vartheta_{(3)}, 1\right) . \quad S$ is the discrete subgroup of the ten-dimensional Poincaré group which corresponds to the crystallographic group of the orbifold geometry. The transformation of the coordinates $\mathbf{Z}=\left(Z^{1}, Z^{2}, Z^{3}, Z^{4}\right)$ under an element $g=$ $\left(\theta^{k}, m_{a} \mathbf{e}_{a}\right) \in S$ is

$$
\mathbf{Z} \stackrel{g}{\mapsto} \theta^{k} \mathbf{Z}+\pi m_{a} \mathbf{e}_{a} .
$$

The multiplication rule of the group (17) is

$$
\left(\theta^{k}, m_{a} \mathbf{e}_{a}\right)\left(\theta^{\tilde{k}}, \tilde{m}_{a} \mathbf{e}_{a}\right)=\left(\theta^{k+\tilde{k}}, m_{a} \mathbf{e}_{a}+\theta^{k} \tilde{m}_{a} \mathbf{e}_{a}\right) \in S,
$$

and inverse elements are given by

$$
\left(\theta^{k}, m_{a} \mathbf{e}_{a}\right)^{-1}=\left(\theta^{-k},-\theta^{-k} m_{a} \mathbf{e}_{a}\right) .
$$

For later usage we also introduce the 'conjugacy class' $[g]$ of an element $g=\left(\theta^{k}, m_{a} \mathbf{e}_{a}\right)$,

$$
[g]=\left\{h g h^{-1} \mid h \in S\right\}=\left\{\left(\theta^{k}, \theta^{\tilde{k}} m_{a} \mathbf{e}_{a}+\left(\mathbb{1}-\theta^{k}\right) \tilde{m}_{a} \mathbf{e}_{a}\right) \mid \tilde{k}, \tilde{m}_{a} \in \mathbb{Z}\right\} .
$$

The set of vectors $\left(\mathbb{1}-\theta^{k}\right) m_{a} \mathbf{e}_{a}$ which appear in the bracket is denoted as

$$
\Lambda_{k} \equiv\left(\mathbb{1}-\theta^{k}\right) \Lambda
$$

and often referred to as the 'sub-lattice' associated with the twist $\theta^{k}$.

With the identification of the space group (17) the definition of the orbifold in (7) becomes clear: It is a subset of $\mathbb{C}^{3}$ onto which every point can be mapped by group transformations (18), $\mathcal{M}_{6}=\mathbb{C}^{3} / S$. Such a subset is also called the 'fundamental domain', and all information of the full space is contained in this region. The fundamental domain itself is by definition invariant under all isometries. 


\section{Fixed points and fixed planes}

The crucial feature of orbifold constructions is the appearance of 'fixed points' and 'fixed planes', or 'fixed tori'. They are points $\mathbf{Z}_{g}$ which are invariant under the action of a space group element $g=\left(\theta^{k}, m_{a} \mathbf{e}_{a}\right) \neq(\mathbb{1}, 0)$,

$$
\mathbf{Z}_{g}=g \mathbf{Z}_{g}=\theta^{k} \mathbf{Z}_{g}+\pi m_{a} \mathbf{e}_{a} .
$$

Fixed points correspond to localization in all six internal dimensions. Fixed tori on the other hand describe invariance of a full torus $T^{2}$ under the twist, and localization in the other two complex dimensions. This situation will occur regularly in the example of relevance for this work. In general, the existence of invariant points or planes under isometry transformations makes the crucial difference between toroidal and orbifold compactifications, as we will see in the following.

Any element $g=\left(\theta^{k}, m_{a} \mathbf{e}_{a}\right)$ which does not act like a pure translation $Z^{i} \stackrel{g}{\mapsto} Z^{i}+\pi m_{a} e_{a}^{i}$ in one of the planes fulfills a fixed point equation (23). This is demonstrated in Appendix A.1, where the explicit solutions for $\mathbf{Z}_{g}$ are constructed.

However, not all space group elements generate independent fixed points. The latter are only defined up to the conjugacy class of the generating element $g$. This can be seen by considering two fixed points $\mathbf{Z}_{g_{1}}, \mathbf{Z}_{g_{2}}$ with generating elements $g_{1}, g_{2}$, respectively, and $g_{2}=h^{-1} g_{1} h$ for a $h \in S$. Then

$$
h \mathbf{Z}_{g_{2}}=h g_{2} \mathbf{Z}_{g_{2}}=g_{1} h \mathbf{Z}_{g_{2}},
$$

which states that $h \mathbf{Z}_{g_{2}}$ fulfills the fixed point equation corresponding to $g_{1}$. Therefore the two fixed points $\mathbf{Z}_{g_{1}}$ and $\mathbf{Z}_{g_{2}}$ are related by a symmetry transformation and thus identical on the orbifold. This observation will lead to crucial projection conditions for physical states after quantization.

\subsubsection{Orbifold boundary conditions}

Heterotic string theory has only closed string solutions. This means that the string fields, which are functions of the world-sheet coordinates $\tau$ and $\sigma$, must obey appropriate boundary conditions. In our conventions they relate the field values at $\sigma$ to the ones at $\sigma+\pi$.

\section{Bosonic coordinates}

Since the orbifold geometry identifies all points which are related by space group transformations (18), the boundary conditions for the coordinate fields read

$$
\mathbf{Z}(\tau, \sigma+\pi)=g \mathbf{Z}(\tau, \sigma)=\theta^{k} \mathbf{Z}(\tau, \sigma)+\pi m_{a} \mathbf{e}_{a} .
$$

Here $g=\left(\theta^{k}, m_{a} \mathbf{e}_{a}\right) \in S$ labels different possible boundary conditions, corresponding to different localization properties of the string. It is already apparent that for non-trivial $g$ the constant part of the solutions will be given by the corresponding fixed point coordinate, suggesting that the string winds around that point and is trapped to its vicinity. 
For later convenience, we can make the ambiguities of the phases in (25) explicit. We introduce a vector of positive frequencies $\omega=\left(\omega^{1}, \omega^{2}, \omega^{3} ; 0\right)$,

$$
\omega^{i}=k v^{i} \bmod 1, \quad 0<\omega^{i} \leqslant 1, \quad i=1, \ldots, 3,
$$

and rewrite the boundary condition as

$$
Z^{i}(\tau, \sigma+\pi)=e^{2 \pi i\left(\tilde{n}^{i}+\omega^{i}\right)} Z^{i}(\tau, \sigma)+\pi m_{a} e_{a}^{i}, \quad \tilde{\mathbf{n}}=\left(\tilde{n}^{1}, \ldots, \tilde{n}^{4}\right) \in \mathbb{Z}^{4} .
$$

The appearing combinations $\tilde{n}^{i}+\omega^{i}$ will appear as fractional frequencies in the mode expansion of the on-shell fields $\mathbf{Z}$, and carry over to excitations after quantization.

\section{Fermions and bosonized fermions}

For the fermionic fields in complex notation,

$$
\psi^{j}\left(\sigma_{-}\right) \equiv \frac{1}{\sqrt{2}}\left(\psi_{R}^{2 j-1}\left(\sigma_{-}\right)+i \psi_{R}^{2 j}\left(\sigma_{-}\right)\right), \quad j=1, \ldots, 4,
$$

the transformation under a space group element $g=\left(\theta^{k}, m_{a} \mathbf{e}_{a}\right)$ is

$$
\psi \stackrel{g}{\mapsto} \theta^{k} \psi
$$

where $\psi \equiv\left(\psi^{1}, \psi^{2}, \psi^{3}, \psi^{4}\right)$. Note that invariance of the term $\psi^{i} \partial_{+} \psi_{i}$ in the action (1) forbids the appearance of shifts for fermionic fields. This leads to

$$
\begin{array}{ll}
\psi\left(\sigma_{-}-\pi\right)=\theta^{k} \psi\left(\sigma_{-}\right) & (\mathrm{R}), \\
\psi\left(\sigma_{-}-\pi\right)=-\theta^{k} \psi\left(\sigma_{-}\right) &
\end{array}
$$

where (R) and (NS) denote Ramond and Neveu-Schwarz boundary conditions, respectively.

It is instructive to represent the fermionic degrees of freedom $\psi^{i}$ by bosonic fields $H^{i}$,

$$
\psi^{i}\left(\sigma_{-}\right) \equiv e^{-2 i H^{i}\left(\sigma_{-}\right)}, \quad \psi^{* i}\left(\sigma_{-}\right) \equiv e^{2 i H^{i}\left(\sigma_{-}\right)},
$$

which implies a transformation rule

$$
H^{i}\left(\sigma_{-}\right) \stackrel{g}{\mapsto} H^{i}\left(\sigma_{-}\right)-\pi k v^{i}
$$

and thus with $\mathbf{M}=\left(M^{1}, M^{2}, M^{3}, M^{4}\right)$ leads to the following boundary conditions:

$$
\begin{array}{lll}
H^{i}\left(\sigma_{-}-\pi\right)=H^{i}\left(\sigma_{-}\right)-\pi\left(k v^{i}+M^{i}\right) & \quad, \quad \mathbf{M} \in \mathbb{Z}^{4} \\
H^{i}\left(\sigma_{-}-\pi\right)=H^{i}\left(\sigma_{-}\right)-\pi\left(k v^{i}+M^{i}+\frac{1}{2}\right), & \mathbf{M} \in \mathbb{Z}^{4} & (\mathrm{NS}) .
\end{array}
$$

These conditions can be expressed by a single equation,

$$
\mathbf{H}_{R}\left(\sigma_{-}-\pi\right)=\mathbf{H}_{R}\left(\sigma_{-}\right)-\pi(\mathbf{q}+k \mathbf{v}), \quad \mathbf{q} \in \Lambda_{\mathrm{SO}(8)}^{*},
$$


where $\Lambda_{\mathrm{SO}(8)}^{*}$ denotes the weight lattice of $\mathrm{SO}(8)$. In a canonical basis the latter is spanned by elements $\mathbf{q}$,

$$
\Lambda_{\mathrm{SO}(8)}^{*}: \quad \mathbf{q}=\left(q^{1}, q^{2}, q^{3}, q^{4}\right), \quad\left\{\begin{array}{c}
q^{i} \in \mathbb{Z}, \quad \sum_{i} q^{i} \text { odd }, \\
q^{i} \in \mathbb{Z}+\frac{1}{2}, \quad \sum_{i} q^{i} \text { even } .
\end{array}\right.
$$

After quantization, this will imprint information about the transformation behavior under the unbroken subgroup of the little group $\mathrm{SO}(8)$ onto the right-moving states.

\section{Gauge fields}

For a consistent string theory it is generically required that the space group acts not only on the coordinate fields, but also on the gauge degrees of freedom $X_{L}^{I}$. This can be understood as a 'gauge embedding' $S \subset G_{10}$, expressed by a mapping

$$
g=\left(\theta^{k}, m_{a} \mathbf{e}_{a}\right) \mapsto\left(k V, m_{a} W_{a}\right),
$$

with 'shift vector' $\mathbf{V}=\left(V^{1}, \ldots, V^{16}\right)$ and 'discrete Wilson lines' [32] $\mathbf{W}_{a}=\left(W_{a}^{1}, \ldots, W_{a}^{16}\right)$. The transformation under the space group element $g$ is then given by

$$
X_{L}^{I} \stackrel{g}{\mapsto} X_{L}^{I}+\pi\left(k V^{I}+m_{a} W_{a}^{I}\right), \quad I=1, \ldots, 16 .
$$

The embedding (38) is not arbitrary. First, $\mathbf{V}$ and $\mathbf{W}_{a}$ are of finite order $N$ and $N_{(a)}$, respectively,

$$
N \mathbf{V} \in \Lambda_{E_{8} \times E_{8}}, \quad N_{(a)} \mathbf{W}_{a} \in \Lambda_{E_{8} \times E_{8}} .
$$

Here $\Lambda_{E_{8} \times E_{8}}$ denotes the root lattice of $E_{8} \times E_{8}$ and there is no summation over the index a. Note that the $X_{L}^{I}$ are only defined up to elements of $\Lambda_{\mathrm{E}_{8} \times \mathrm{E}_{8}}$, by construction of the heterotic string, and therefore the right-hand side of each of the conditions expresses trivial action on the fields $X_{L}^{I}$.

For each $a=1, \ldots, 6$, the integer $N_{(a)}$ denotes the 'order of the lattice vector $\mathbf{e}_{a}$ '. It is a divisor of the order $N$ of the orbifold which is determined by the equation $\left(\theta, \mathbf{e}_{a}\right)^{N_{(a)}}=$ $\left(\theta^{N_{(a)}}, 0\right)$. This states that for $N_{(a)}$ repeated transformations the effect of the lattice shift is trivial, as long as twists are involved. For the Wilson lines this translates into (40).

Further conditions on the Wilson lines arise if two lattice shifts are equivalent on the orbifold, $\left(\mathbb{1}, \mathbf{e}_{a}\right) \in\left[\left(\mathbb{1}, \mathbf{e}_{b}\right)\right]$. Then also the two associated Wilson lines have to agree up to root lattice elements. This restricts the number of distinct Wilson lines in a given orbifold geometry.

Second, there are consistency conditions from string theory, namely the requirement that the partition function is modular invariant $[30,41,59]$,

$$
N\left(\mathbf{V}^{2}-\mathbf{v}^{2}\right)=0 \bmod 2 .
$$


As a consequence, the gauge embedding is generically non-trivial. Except for special situations, like for example a $\mathbb{Z}_{3}$ orbifold with $\mathbf{v}=(1 / 3,-2 / 3,1 / 3 ; 0)$, this condition implies a non-vanishing shift vector $\mathbf{V}$. However, a large ambiguity remains in the definition of the gauge embeddings (38), especially for the choice of Wilson lines. This gave rise to systematic scans over parts of the heterotic orbifold landscape [14].

With non-vanishing Wilson lines, the above conditions generalize. Here we state them in the form of 'strong modular invariance conditions' ${ }^{6}$,

$$
\begin{aligned}
\frac{1}{2}\left(\mathbf{V}^{2}-\mathbf{v}^{2}\right) & =0 \bmod 1, \\
\mathbf{V} \cdot \mathbf{W}_{a} & =0 \bmod 1, \\
\mathbf{W}_{a} \cdot \mathbf{W}_{b} & =0 \bmod 1 \quad \text { for } \mathbf{W}_{a} \neq \mathbf{W}_{b}, \\
\frac{1}{2} \mathbf{W}_{a}^{2} & =0 \bmod 1 .
\end{aligned}
$$

The resulting boundary conditions for the fields $X_{L}^{I}$ read

$$
X_{L}^{I}\left(\sigma_{+}+\pi\right)=X_{L}^{I}\left(\sigma_{+}\right)+\pi\left(p^{I}+k V^{I}+m_{a} W_{a}^{I}\right), \quad \mathbf{p}=\left(p^{1}, \ldots, p^{16}\right) \in \Lambda_{\mathrm{E}_{8} \times \mathrm{E}_{8}} .
$$

The appearance of the vector $\mathbf{p}$ in the boundary conditions of the left-movers is crucial for all what follows. After quantization it will determine the transformation behavior of a state with respect to the present gauge symmetry.

\subsubsection{Classical solutions}

The equations of motion (6) have straightforward solutions ${ }^{7}(2)$,

$$
Z^{i}(\tau, \sigma)=Z_{L}^{i}\left(\sigma_{+}\right)+Z_{R}^{i}\left(\sigma_{-}\right), \quad \psi^{i}(\tau, \sigma)=e^{-2 i H^{i}\left(\sigma_{-}\right)}, \quad X^{I}(\tau, \sigma)=X_{L}^{I}\left(\sigma_{+}\right),
$$

for $i=1, \ldots, 4, I=1, \ldots, 16$. From the boundary conditions (27), (36) and (43) one can read off the mode expansions (recall that $\omega^{i}=k v^{i} \bmod 1 \geqslant 0$ ):

$$
\begin{aligned}
& Z_{L}^{i}\left(\sigma_{+}\right)=f_{L}^{i}\left(\sigma_{+}\right)+\frac{i}{2} \sum_{\tilde{\nu}_{\mathrm{sh}}^{i} \neq 0} \frac{1}{\tilde{\nu}_{\mathrm{sh}}^{i}} \tilde{\alpha}_{\tilde{\nu}_{\mathrm{sh}}^{i}}^{i} e^{-2 i \tilde{\nu}_{\mathrm{sh}}^{i} \sigma_{+}}, \quad \tilde{\nu}_{\mathrm{sh}} \equiv \tilde{\mathbf{n}}-\omega, \quad \tilde{\mathbf{n}} \in \mathbb{Z}^{4}, \\
& Z_{R}^{i}\left(\sigma_{-}\right)=f_{R}^{i}\left(\sigma_{-}\right)+\frac{i}{2} \sum_{\nu_{\mathrm{sh}}^{i} \neq 0} \frac{1}{\nu_{\mathrm{sh}}^{i}} \alpha_{\nu_{\mathrm{sh}}^{i}}^{i} e^{-2 i \nu_{\mathrm{sh}}^{i} \sigma_{-}}, \quad \nu_{\mathrm{sh}} \equiv \mathbf{n}+\omega, \quad \mathbf{n} \in \mathbb{Z}^{4}, \\
& H^{i}\left(\sigma_{-}\right)=h^{i}+q_{\mathrm{sh}}^{i} \sigma_{-}+\frac{i}{2} \sum_{m \neq 0} \frac{1}{m} \beta_{m}^{i} e^{-2 i m \sigma_{-}}, \quad \mathbf{q}_{\mathrm{sh}} \equiv \mathbf{q}+k \mathbf{v}, \quad \mathbf{q} \in \Lambda_{\mathrm{SO}(8)}^{*}, \\
& X_{L}^{I}\left(\sigma_{+}\right)=x^{I}+p_{\mathrm{sh}}^{I} \sigma_{+}+\frac{i}{2} \sum_{\tilde{m} \neq 0} \frac{1}{\tilde{m}} \tilde{\alpha}_{\tilde{m}}^{I} e^{-2 i \tilde{m} \sigma_{+}}, \quad \mathbf{p}_{\mathrm{sh}} \equiv \mathbf{p}+\mathbf{V}_{g}, \quad \mathbf{p} \in \Lambda_{\mathrm{E}_{8} \times \mathrm{E}_{8}}, \\
& \mathbf{V}_{g} \equiv k \mathbf{V}+m_{a} \mathbf{W}_{a} .
\end{aligned}
$$

\footnotetext{
${ }^{6}$ The counterparts are 'weak modular invariance conditions', as discussed in [18]. Solutions of the weak modular invariance conditions also fulfill the strong modular invariance conditions, after adding appropriate elements of $\Lambda_{\mathrm{E}_{8} \times \mathrm{E}_{8}}$.

${ }^{7}$ Contributions $X_{R}^{I}\left(\sigma_{-}\right)$are forbidden by world sheet supersymmetry.
} 
Similar solutions apply for the conjugate coordinate fields $Z_{L}^{* i}, Z_{R}^{* i}, \psi_{R}^{* i}$. The appearing constants $\tilde{\alpha}_{\hat{q}_{\mathrm{sh}}^{i}}^{i}, \tilde{\alpha}_{\hat{q}_{\mathrm{sh}}^{* i}}^{* i}, \alpha_{\hat{r}_{\mathrm{sh}}^{i}}^{i}, \alpha_{\hat{r}_{\mathrm{sh}}^{* i}}^{* i}, h^{i}, \beta_{n}^{i}, x^{I}, \tilde{\alpha}_{n}^{I}$ depend on the space group element $g=\left(\theta^{k}, m_{a} \mathbf{e}_{a}\right)$ that defines the boundary condition. This is also the case for the functions $f_{L}^{i}\left(\sigma_{+}\right), f_{R}^{i}\left(\sigma_{-}\right)$,

$$
\begin{aligned}
& f_{L}^{i}\left(\sigma_{+}\right)=\left\{\begin{array}{ll}
\frac{1}{2} z^{i}+\frac{1}{2}\left(p^{i}+m_{a} e_{a}^{i}\right) \sigma_{+}, & \text {torus } T^{i} \text { is invariant }\left(\vartheta_{(i)}^{k}=1\right) \\
\frac{1}{2} z_{g}^{i}, & \text { torus } T^{i} \text { has a fixed point } z_{g}^{i}
\end{array},\right. \\
& f_{R}^{i}\left(\sigma_{-}\right)=\left\{\begin{array}{ll}
\frac{1}{2} z^{i}+\frac{1}{2}\left(p^{i}-m_{a} e_{a}^{i}\right) \sigma_{-}, & \text {torus } T^{i} \text { is invariant }\left(\vartheta_{(i)}^{k}=1\right) \\
\frac{1}{2} z_{g}^{i}, & \text { torus } T^{i} \text { has a fixed point } z_{g}^{i}
\end{array},\right.
\end{aligned}
$$

and their conjugates $f_{L}^{* i}=\left(f_{L}^{i}\right)^{*}, f_{R}^{* i}=\left(f_{R}^{i}\right)^{*}$. Here $z^{i}$ and $p^{i}$ are arbitrary constants, they describe a free center-of-mass motion of the string over the bulk of the torus $T^{i}$.

These solutions contain the localization properties of the string. We distinguish between 'untwisted' strings, defined by $g=(\mathbb{1}, 0)$, and 'twisted' strings, which have $\theta^{k} \neq \mathbb{1}$ and fulfill a non-trivial fixed point (or fixed torus) equation $g \mathbf{z}_{g}=\mathbf{z}_{g}$. Equations (46), (47) show that untwisted strings are completely delocalized over the full geometry. In contrast, twisted strings cannot move freely, they are localized at least in one of the internal tori.

\subsection{Quantization and the low-energy spectrum}

\subsubsection{The Hilbert space}

With the explicit mode expansions from the last section canonical quantization in lightcone gauge is now straightforward. Here we first summarize the results before we discuss conditions on physical states due to the requirement of compatibility with the underlying orbifold.

The states of the quantized theory follow from promoting the appearing coefficients to operators which obey appropriate canonical commutation relations. Each fixed point on the orbifold is associated to a class of space group elements $[g]$ and gives rise to distinct boundary conditions. Thus the coefficients of the corresponding mode expansion and hence also the operators of the quantum theory depend on this conjugacy class. They are defined on an associated Hilbert space $\mathcal{H}_{[g]}$, and the total Hilbert space is of the form

$$
\mathcal{H}=\bigoplus_{[g]} \mathcal{H}_{[g]}
$$

where the direct sum is taken over all disjoint conjugacy classes $[g] \subset S$. Each contribution $\mathcal{H}_{[g]}$ is referred to as a 'sector'. We stress that inequivalent solutions of the classical field equations will thus give rise to distinct states in the effective field theory after quantization, with distinct localization properties and charges.

All states in a sector $\mathcal{H}_{[g]}$ are subject to projections which define the physical subset of states. They are 'level matching conditions', which ensure that the masses of left-moving states are equal to the ones of right-moving states, orbifold consistency conditions, which we will discuss in some detail later, and 'GSO projections' [60] for the fermions. The latter 
guarantee that there are as many bosons as fermions in the theory. In practice, it restricts the quantum numbers $\mathbf{q}$ to lie either in the integer lattice of $\Lambda_{\mathrm{SO}(8)}^{*}$ (cf. the upper line of (37)) or the half-integer lattice (cf. the lower line of (37)).

Operators which correspond to positive (negative) frequencies are understood as creation (annihilation) operators,

$$
\tilde{\alpha}_{n}^{i}, \tilde{\alpha}_{n}^{* i}, \alpha_{n}^{i}, \alpha_{n}^{* i}, \tilde{\alpha}_{n}^{I}, \beta_{n}^{i}: \begin{cases}n<0 & \text { creation operator } \\ n>0 & \text { annihilation operator }\end{cases}
$$

and ground states of the sector $\mathcal{H}_{[g]}$ are defined as states which vanish upon application of any of the annihilation operators. They carry information about the internal discrete momenta $\mathbf{p}_{\mathrm{sh}}$ and $\mathbf{q}_{\mathrm{sh}}$ of left- and right-movers, respectively:

$$
\left|\mathbf{p}_{\mathrm{sh}}, \mathbf{q}_{\mathrm{sh}}\right\rangle_{[g]} \equiv\left|\mathbf{p}_{\mathrm{sh}}\right\rangle_{L} \otimes\left|\mathbf{q}_{\mathrm{sh}}\right\rangle_{R} \otimes|\chi\rangle_{[g]}
$$

Here $|\chi\rangle_{[g]}$ unifies a set of quantum numbers which are related to the localization of the state and will be specified shortly.

A general state in $\mathcal{H}_{[g]}$ now takes the form $\left(n_{i}, m_{i}, p_{i}, q_{i}, r_{i}, u_{i}<0\right)$

$$
\underbrace{\tilde{\alpha}_{n_{1}}^{i_{1}} \cdots \tilde{\alpha}_{n_{N}}^{i_{N}}}_{\text {from } Z_{L}^{i}} \underbrace{\tilde{\alpha}_{m_{1}}^{* j_{1}} \cdots \tilde{\alpha}_{m_{M}}^{* j_{M}}}_{\text {from } Z_{L}^{* i}} \underbrace{\alpha_{p_{1}}^{k_{1}} \cdots \alpha_{p_{P}}^{k_{P}}}_{\text {from } Z_{R}^{i}} \underbrace{\alpha_{q_{1}}^{* l_{1}} \cdots \alpha_{q_{Q}}^{* l_{Q}}}_{\text {from } Z_{R}^{* i}} \underbrace{\tilde{\alpha}_{r_{1}}^{I_{1}} \cdots \tilde{\alpha}_{r_{R}}^{I_{R}}}_{\text {from } Z_{L}^{I}} \underbrace{\beta_{u_{1}}^{s_{1}} \cdots \beta_{u_{U}}^{s_{U}}}_{\text {from } H_{R}^{i}}\left|\mathbf{p}_{\mathrm{sh}}, \mathbf{q}_{\mathrm{sh}}\right\rangle_{[g]} .
$$

The oscillator numbers of excited states are counted by the number operators ${ }^{8}$ (here no $i$-summation)

$$
\tilde{N}^{i}=\frac{1}{\omega^{i}} \sum_{\tilde{\nu}_{\mathrm{sh}}^{i}>0} \tilde{\alpha}_{-\tilde{\nu}_{\mathrm{sh}}^{i}}^{i} \tilde{\alpha}_{\tilde{\nu}_{\mathrm{sh}}^{i}}^{* i}, \quad \tilde{N}^{* i}=\frac{1}{\bar{\omega}^{i}} \sum_{\tilde{\nu}_{\mathrm{sh}}^{* i}>0} \tilde{\alpha}_{-\tilde{\nu}_{\mathrm{sh}}^{* i}}^{* i} \tilde{\alpha}_{\tilde{\nu}_{\mathrm{sh}}^{* i}}^{i},
$$

and similarly for $N^{i}, N^{* i}$. Here $\bar{\omega}^{i}, \tilde{\nu}_{\mathrm{sh}}^{* i}$ are defined similar to $\omega^{i}, \tilde{\nu}_{\mathrm{sh}}^{i}$, with replacement $k \rightarrow-k$. These number operators have non-negative integer eigenvalues, also denoted by $N^{i}, N^{* i}, \tilde{N}^{i}, \tilde{N}^{* i}$ in the following. The string theory origins of the appearing quantum numbers of a state are summarized in Figure 1.

\subsubsection{Orbifold compatibility}

Equivalent boundary conditions $g$ and $h g h^{-1}, g, h \in S$, lead to the same Hilbert space $\mathcal{H}_{[g]}$, since on the orbifold the corresponding fixed points are identical (cf. Equation (24)). Explicitly,

$$
\begin{aligned}
\mathbf{Z}(\tau, \sigma+\pi) & =g \mathbf{Z}(\tau, \sigma), \\
h \mathbf{Z}(\tau, \sigma+\pi) & =\left(h g h^{-1}\right) h \mathbf{Z}(\tau, \sigma)
\end{aligned}
$$

\footnotetext{
${ }^{8}$ Only the notion of those which are associated with the coordinate fields $Z$ will be needed later.
} 
have to lead to the same physical states, and likewise for the other fields. This imposes important consistency conditions on the physical states in $\mathcal{H}_{[g]}$,

$$
h \mid \text { phys }\rangle=\mid \text { phys }\rangle \quad \text { for all } h \in S .
$$

In the following, we will construct these states explicitly. For that we first calculate a phase $\Phi_{h}$ which arises due to properties of the solutions (45). Then we will require that this phase is one for physical states, thereby defining the orbifold projection.

For each $g=\left(\theta^{k}, m_{a} \mathbf{e}_{a}\right)$ one can solve the equations of motion with boundary conditions given by (53a). After canonical quantization they give rise to Hilbert spaces $\mathcal{H}_{g}$, with ground states

$$
\left|\mathbf{p}_{\mathrm{sh}}, \mathbf{q}_{\mathrm{sh}}\right\rangle_{g}=\left|\mathbf{p}_{\mathrm{sh}}\right\rangle_{L} \otimes\left|\mathbf{q}_{\mathrm{sh}}\right\rangle_{R} \otimes\left|\chi_{g}\right\rangle
$$

where $\left|\chi_{g}\right\rangle=\left|\chi_{g}^{1}\right\rangle \otimes \cdots \otimes\left|\chi_{g}^{4}\right\rangle$ collects quantum numbers which specify the localization properties of the state:

$$
\left|\chi_{g}^{i}\right\rangle= \begin{cases}\left|p^{i}, m_{a} e_{a}^{i}\right\rangle, & T^{i} \text { is a fixed torus }\left(\vartheta_{(i)}^{k}=1\right) \\ \left|z_{g}^{i}\right\rangle, & T^{i} \text { has a fixed point } z_{g}^{i}\end{cases}
$$

Thus the states in $\mathcal{H}_{g}$ either have center-of-mass momentum $p^{i}$ and possibly winding numbers $m_{a}$ along the directions $e_{a}^{i}$, or they are localized at the fixed point coordinate $z_{g}^{i}$ (compare to (46)).

In the former case, non-vanishing momenta and winding modes will induce massive states in the effective low-energy theory. For example, the momenta $p^{i}$ take discrete values on the compact torus, which directly map to the Kaluza-Klein mass of the state in the effective field theory after dimensional reduction. Assuming a compactification scale much above the low-energy scale, we can ignore such contributions and restrict to $p^{i}=m_{a} e_{a}^{i}=0$ for bulk states of the plane $i$.

To keep the notation short in the following, we further introduce the 'local twist vector' $\mathbf{v}_{g}$ and the 'local shift vector' $\mathbf{V}_{g}$, which already appeared in (45),

$$
\mathbf{v}_{g} \equiv k \mathbf{v}, \quad \mathbf{V}_{g} \equiv k \mathbf{V}+m_{a} \mathbf{W}_{a},
$$

such that $\mathbf{q}_{\mathrm{sh}}=\mathbf{q}+\mathbf{v}_{g}, \mathbf{p}_{\mathrm{sh}}=\mathbf{p}+\mathbf{V}_{g}$.

\section{Transformation rules for states}

A general space group element $h$ has non-trivial action on the states of $\mathcal{H}_{g}$. The constant parts of $H_{R}^{i}, X_{L}^{I}$ transform by shifts, cf. (33), (39),

$$
h^{i} \stackrel{h}{\mapsto} h^{i}-\pi v_{h}^{i}, \quad \quad x^{I} \stackrel{h}{\mapsto} x^{I}+\pi V_{h}^{I} .
$$

For momentum eigenstates this corresponds to the following transformation behavior:

$$
\left|\mathbf{q}_{\mathrm{sh}}\right\rangle_{R} \stackrel{h}{\mapsto} e^{-2 \pi i \mathbf{q}_{\mathrm{sh}} \cdot \mathbf{v}_{h}}\left|\mathbf{q}_{\mathrm{sh}}\right\rangle_{R}, \quad \quad\left|\mathbf{p}_{\mathrm{sh}}\right\rangle_{L} \stackrel{h}{\mapsto} e^{2 \pi i \mathbf{p}_{\mathrm{sh}} \cdot \mathbf{V}_{h}}\left|\mathbf{p}_{\mathrm{sh}}\right\rangle_{L} .
$$


From the explicit mode expansions (45) one can infer the transformation properties of the creation and annihilation operators, for example

$$
\tilde{\alpha}_{\hat{q}_{\mathrm{sh}}^{i}}^{i} \stackrel{h}{\mapsto} e^{2 \pi i v_{h}^{i}} \tilde{\alpha}_{\hat{q}_{\mathrm{sh}}^{i}}^{i}, \quad \quad \tilde{\alpha}_{\hat{q}_{\mathrm{sh}}^{* i}}^{* i} \stackrel{h}{\mapsto} e^{-2 \pi i v_{h}^{i}} \tilde{\alpha}_{\hat{q}_{\mathrm{sh}}^{* i}}^{* i}
$$

The corresponding excitations are counted by the integer vectors $\tilde{\mathbf{N}}=\left(\tilde{N}^{1}, \ldots, \tilde{N}^{4}\right)$ and similarly $\tilde{\mathbf{N}}^{*}, \mathbf{N}, \mathbf{N}^{*}$.

However, as we will see the relevant states for the low-energy effective theory have $\mathbf{N}=\mathbf{N}^{*}=0$, and we shall restrict to that case from now on. It is then convenient to introduce a vector $\mathbf{R}=\left(R^{1}, \ldots, R^{4}\right)$,

$$
R^{i} \equiv q_{\mathrm{sh}}^{i}+\tilde{N}^{* i}-\tilde{N}^{i}
$$

where the $R^{i}$ differ for bosons and fermions in a multiplet, as we shall discuss in more detail later. A state with oscillator numbers can then be expressed as $\left|\mathbf{p}_{\mathrm{sh}}, \mathbf{R}\right\rangle \equiv\left|\mathbf{p}_{\mathrm{sh}}, \mathbf{q}_{\mathrm{sh}} ; \tilde{\mathbf{N}}, \tilde{\mathbf{N}}^{*}\right\rangle$,

$$
\left|\mathbf{p}_{\mathrm{sh}}, \mathbf{R}\right\rangle=\tilde{\alpha}_{n_{1}}^{i_{1}} \cdots \tilde{\alpha}_{m_{1}}^{* j_{1}} \cdots\left|\mathbf{p}_{\mathrm{sh}}\right\rangle_{L} \otimes\left|\mathbf{q}_{\mathrm{sh}}\right\rangle_{R}
$$

with transformation behavior

$$
\left|\mathbf{p}_{\mathrm{sh}}, \mathbf{R}\right\rangle \stackrel{h}{\mapsto} e^{2 \pi i\left(\mathbf{p}_{\mathrm{sh}} \cdot \mathbf{V}_{h}-\mathbf{R} \cdot \mathbf{v}_{h}\right)}\left|\mathbf{p}_{\mathrm{sh}}, \mathbf{R}\right\rangle
$$

Next we consider the transformation behavior of the center-of-mass contribution $\left|\chi_{g}\right\rangle$, defined in (56). If $g$ has a fixed point $\mathbf{z}_{g}$, then $h$ maps this to a fixed point of the conjugated element (cf. (24)),

$$
\left|\mathbf{z}_{g}\right\rangle \stackrel{h}{\mapsto}\left|\mathbf{z}_{h g h^{-1}}\right\rangle .
$$

In general, this causes an inconsistency: For $[g, h] \neq 0$ the state on the right hand side is not contained in $\mathcal{H}_{g}$. This problem is solved in $\mathcal{H}_{[g]}$ by considering a superposition of states,

$$
|\chi\rangle_{[g]}=\sum_{l \in[g]} \chi_{l}\left|\mathbf{z}_{l}\right\rangle
$$

with coefficients $\chi_{l}$. The states of interest are eigenstates with respect to the action of all space group elements $h=\left(\theta^{\tilde{k}}, \tilde{m}_{a} \mathbf{e}_{a}\right)$,

$$
|\chi\rangle_{[g]} \stackrel{h}{\mapsto} \tilde{\Phi}_{h}|\chi\rangle_{[g]}, \quad \quad \tilde{\Phi}_{\left(\theta^{\tilde{k}}, \tilde{m}_{a} \mathbf{e}_{a}\right)}=\eta_{\tilde{m}_{a} \mathbf{e}_{a}}^{\prime} \eta_{\tilde{k}}
$$

where the multiplicative form of the phase $\tilde{\Phi}_{h}$ is a consequence of the observation $\left(\theta^{\tilde{k}}, \tilde{m}_{a} \mathbf{e}_{a}\right)=$ $\left(\mathbb{1}, \tilde{m}_{a} \mathbf{e}_{a}\right)\left(\theta^{\tilde{k}}, 0\right)$. Consistency with the group multiplication then further requires for arbitrary $k, k, m_{a}, \tilde{m}_{a}$

$$
\eta_{\left(m_{a}+\tilde{m}_{a}\right) \mathbf{e}_{a}}^{\prime}=\eta_{m_{a} \mathbf{e}_{a}}^{\prime} \eta_{\tilde{m}_{a} \mathbf{e}_{a}}^{\prime}, \quad \eta_{k+\tilde{k}}=\eta_{k} \eta_{\tilde{k}}, \quad \eta_{\theta^{k} m_{a} \mathbf{e}_{a}}^{\prime}=\eta_{m_{a} \mathbf{e}_{a}}^{\prime}
$$


The latter relation follows from the explicit inverse element (20). Note that with (21) it also implies that elements from the same conjugacy class lead to the same geometrical phase, $\tilde{\Phi}_{\tilde{g}}=\tilde{\Phi}_{h \tilde{g} h^{-1}}$.

Every state is invariant under the unit element, $\tilde{\Phi}_{(\mathbb{1}, 0)}=1$. However, also for nonvanishing $\eta_{\tilde{k}}$ there is the possibility that one can find a translation $m_{a}^{\tilde{k}} \mathbf{e}_{a}$ such that there is a cancelation between the two factors, $\eta_{m_{a}^{\tilde{k}} \mathbf{e}_{a}}^{\prime} \eta_{\tilde{k}}=1$. If $g$ itself is among these elements $\left(\theta^{\tilde{k}}, m_{a}^{\tilde{k}} \mathbf{e}_{a}\right)$ in a sector $\mathcal{H}_{[g]}$, invariance of $\left|\mathbf{z}_{g}\right\rangle$ under commuting elements (cf. (64)) is lifted to the superposition $|\chi\rangle_{[g]}$.

In Appendix A.3 we make an explicit ansatz for the superposition (65), and find eigenstates $|\chi\rangle_{[g]}=\left|\gamma^{\prime}, \gamma\right\rangle_{[g]}$ of all $h=\left(\theta^{\tilde{k}}, \tilde{m}_{a} \mathbf{e}_{a}\right) \in S$,

$$
\begin{aligned}
& \left|\gamma^{\prime}, \gamma\right\rangle_{[g]}=\left(\sum_{m_{a}^{\prime} \mathbf{e}_{a} \in \Lambda} e^{2 \pi i k \gamma_{a}^{\prime} m_{a}^{\prime}}\left(\mathbb{1}, m_{a}^{\prime} \mathbf{e}_{a}\right)\right)\left(\sum_{n=0}^{N-1} e^{-2 \pi i n \gamma_{a}^{\prime} m_{a}} e^{-2 \pi i \gamma n}\left(\theta^{n}, 0\right)\right)\left|\mathbf{z}_{g}\right\rangle, \\
& \left|\gamma^{\prime}, \gamma\right\rangle_{[g]} \stackrel{h}{\mapsto} \tilde{\Phi}_{h}\left|\gamma^{\prime}, \gamma\right\rangle_{[g]}, \quad \tilde{\Phi}_{h} \equiv e^{2 \pi i \gamma_{a}^{\prime}\left(\tilde{k} m_{a}-k \tilde{m}_{a}\right)} e^{2 \pi i \gamma \tilde{k}},
\end{aligned}
$$

where $\gamma^{\prime}=\left(\gamma_{1}^{\prime}, \ldots, \gamma_{6}^{\prime}\right)$ and $\gamma$ are constants that characterize the state, and $g=\left(\theta^{k}, m_{a} \mathbf{e}_{a}\right)$. The presence of the transformation phase due to $\gamma^{\prime}$ is a new result and and extension of what is usually done in the literature, cf. $[13,14,49]$, but does not influence the particle spectra calculated there. We will comment more on this in Sections 2.3.2 and 3.2.3.

The constants $\gamma^{\prime}, \gamma$ are constrained by the conditions given in (418) in Appendix A.3. One finds that consequently they must be of finite order. For example

$$
\gamma=\frac{j}{N_{(g)}}, \quad j=1, \ldots, N_{(g)},
$$

where $N_{(g)}$ is defined as the smallest positive integer with

$$
\left(\mathbb{1}-\theta^{N_{(g)}}\right) m_{a} \mathbf{e}_{a} \in \Lambda_{k}, \quad 1 \leqslant N_{(g)} \leqslant k .
$$

Note that $N_{(g)}$ is a divisor of $k$. The $\gamma_{a}^{\prime}$ are related to the geometry of the lattice. Their order corresponds to the order of the associated lattice vector. An explicit example will be discussed in the next chapter.

The contributions $e^{2 \pi i \gamma \tilde{k}}$ to the phase $\tilde{\Phi}_{h}$ are conventionally referred to as 'gammaphases' in the literature. However, we stress that generically a superposition of states from the whole relevant sub-lattice $\Lambda_{k}$ has to be considered in order to guarantee invariance under the full space group $S$. This induces an additional contribution $e^{2 \pi i \gamma_{a}^{\prime}\left(\tilde{k} m_{a}-k \tilde{m}_{a}\right)}$ to the phase $\tilde{\Phi}_{h}$, which does not vanish for all choices of $h=\left(\theta^{\tilde{k}}, \tilde{m}_{a} \mathbf{e}_{a}\right)$.

In summary, in a sector $\mathcal{H}_{[g]}$, where $g=\left(\theta^{k}, m_{a} \mathbf{e}_{a}\right)$, eigenstates with respect to the action of arbitrary space group elements read

$$
\left|\mathbf{p}_{\mathrm{sh}}, \mathbf{R} ; \gamma^{\prime}, \gamma\right\rangle_{[g]}=\left|\mathbf{p}_{\mathrm{sh}}, \mathbf{R}\right\rangle \otimes\left|\gamma^{\prime}, \gamma\right\rangle_{[g]} .
$$


Under transformation with an element $h=\left(\theta^{\tilde{k}}, \tilde{m} \mathbf{e}_{a}\right)$ they pick up a phase $\Phi_{h}$,

$$
\left|\mathbf{p}_{\mathrm{sh}}, \mathbf{R} ; \gamma^{\prime}, \gamma\right\rangle_{[g]} \stackrel{h}{\mapsto} \Phi_{h}\left|\mathbf{p}_{\mathrm{sh}}, \mathbf{R} ; \gamma^{\prime}, \gamma\right\rangle_{[g]}, \quad \quad \Phi_{h}=e^{2 \pi i\left(\mathbf{p}_{\mathrm{sh}} \cdot \mathbf{V}_{h}-\mathbf{R} \cdot \mathbf{v}_{h}\right)} \tilde{\Phi}_{h}
$$

where $\tilde{\Phi}_{h}$ was defined in (69).

\section{Consistency conditions}

The transformation rule (73) describes the effect of a transition from boundary condition $g$ in (53a) to boundary condition $h g h^{-1}$ in $(53 \mathrm{~b})$ on the states of the corresponding quantized theory. Quantitatively, it is given in form of a phase $\Phi_{h}$. However, on the underlying orbifold geometry it is impossible to distinguish between conjugated space group elements. This translates into consistency conditions for physical states in $\mathcal{H}_{[g]}$ :

$$
\mathcal{H}_{[g]}: \quad \Phi_{h}=1 \quad \text { for all } h \in S .
$$

The phase $\Phi$ from (73) can be decomposed into a universal contribution for all superpositions of states at equivalent fixed points, and a $\gamma^{\prime}, \gamma$ dependent geometrical factor $\tilde{\Phi}_{h}$. If the latter phase is trivial, $\tilde{\Phi}_{h}=1$, the consistency condition is a non-trivial constraint for the allowed Lorentz symmetry and gauge symmetry quantum numbers, the familiar 'orbifold projection'. For the superposition (68), this is the case for all commuting elements $[h, g]=0$.

For $h \in S$ with $\tilde{\Phi}_{h} \neq 1$, additional phases contribute to the projection condition $\Phi_{h}=1$. The latter are then often referred to as the 'modified orbifold projection conditions'. From all possible quantum numbers $\left(\mathbf{p}_{\mathrm{sh}}, \mathbf{R}, \gamma^{\prime}, \gamma\right)$ they project out the unphysical combinations. Here the observation is that for any pair of charges $\left(\mathbf{p}_{\mathrm{sh}}, \mathbf{R}\right)$, one can find an eigenstate of $h$ for which the condition is fulfilled, specified by a choice of the finitely many distinct $\gamma^{\prime}, \gamma$. Thus none of these pairs is absent in the physical spectrum, but they are grouped into a finite number of subsets, characterized by labels $\gamma^{\prime}, \gamma$. The latter respect gauge symmetry and supersymmetry, in the sense that all zero modes in a subset form complete multiplets under these symmetries. Let $h \in S$ denote all elements which commute with $g$, and $r \in S$ the rest. Then the above statement can be sketched as

$$
\begin{aligned}
{[h, g]=0 } \\
{[r, g] \neq 0 }
\end{aligned}: \quad\left\{\left(\mathbf{p}_{\mathrm{sh}}, \mathbf{R}\right)\right\} \stackrel{\Phi_{h}=1}{\longmapsto}\left\{\left(\mathbf{p}_{\mathrm{sh}}, \mathbf{R}\right)\right\}_{[g]} \stackrel{\Phi_{r}=1}{\longmapsto}\left\{\left(\mathbf{p}_{\mathrm{sh}}, \mathbf{R} ; \gamma^{\prime}, \gamma\right)\right\}_{[g]} .
$$

Note that ignoring the existence of the translational gamma phases related to $\gamma^{\prime}$ can only yield a spectrum with the correct charge vectors, if for localized states the last step in the above mapping is skipped. However, throughout this work we take the viewpoint that the information on the superposition of states at the infinitely many sub-lattice points is an additional quantum number for physical states, and thus the full set of projection conditions (74) has to be fulfilled in all sectors. 


\subsubsection{Conditions for massless states}

For the construction of an effective field theory as the low-energy limit of the compactified heterotic string only massless states are of interest,

$$
m_{L}^{2}=m_{R}^{2}=0
$$

A state (51) from the sector $\mathcal{H}_{[g]}$ has three contributions to the above masses. One is due to its internal momenta $\mathbf{p}_{\mathrm{sh}}, \mathbf{q}_{\mathrm{sh}}$. The second is the zero-point energy, which has a universal contribution due to the shifts $\omega^{i}$ in the twisted oscillator frequencies:

$$
\delta c=\frac{1}{2} \sum_{i=1}^{3} \omega^{i}\left(1-\omega^{i}\right) .
$$

Third, oscillator excitations lead to mass contributions, given by the fractional oscillator numbers

$$
\tilde{N}=\sum_{i=1}^{3}\left(\omega^{i} \tilde{N}^{i}+\bar{\omega}^{i} \tilde{N}^{* i}\right), \quad N=\sum_{i=1}^{3}\left(\omega^{i} N^{i}+\bar{\omega}^{i} N^{* i}\right)
$$

The resulting conditions for massless states (76) are

$$
\begin{aligned}
& \frac{1}{8} m_{L}^{2}=\frac{1}{2} \mathbf{p}_{\mathrm{sh}}^{2}-1+\delta c+\tilde{N}=0, \\
& \frac{1}{8} m_{R}^{2}=\frac{1}{2} \mathbf{q}_{\mathrm{sh}}^{2}-\frac{1}{2}+\delta c+N=0 .
\end{aligned}
$$

These equations formulate further conditions for states which appear in the low-energy effective field theory. All other states will have masses of the order of the string scale, and will be neglected in the analysis.

\subsubsection{The generic spectrum}

In general, the space group $S$ acts on all three complex internal dimensions $i=1,2,3$. The evaluation of the mass conditions (79), (80) and the orbifold projections (74) for all sectors yields all zero modes which are fully compatible with the compactification. Thus the resulting spectrum defines an effective theory in four dimensions, assuming that the volume of all internal tori is small. Here we give a brief overview over generic features of the low-energy spectrum of heterotic orbifold models.

\section{The gravity sector}

Gravity lives in the ten-dimensional bulk. Thus corresponding states arise from the untwisted sector $\mathcal{H}_{(\mathbb{1}, 0)}$. Furthermore, these states are uncharged under gauge symmetries, 
$\mathbf{p}=0$, do not involve superpositions, $\gamma^{\prime}=\gamma=0$, and include two-tensors with respect to Lorentz symmetry. The relevant solutions of the mass equations (79), (80) are

$$
|\mathbf{q}\rangle_{R} \otimes \tilde{\alpha}_{-1}^{i}|0\rangle_{L}, \quad|\mathbf{q}\rangle_{R} \otimes \tilde{\alpha}_{-1}^{* i}|0\rangle_{L},
$$

with

$$
\mathbf{q}=\underbrace{( \pm 1,0,0,0)}_{\mathbf{8}_{v} \text { of } \mathrm{SO}(8)} \text { or } \mathbf{q}=\underbrace{ \pm\left(\frac{1}{2}, \frac{1}{2}, \frac{1}{2}, \frac{1}{2}\right),\left(\frac{1}{2}, \frac{1}{2},-\frac{1}{2},-\frac{1}{2}\right)}_{\mathbf{8}_{s} \text { of } \mathrm{SO}(8)},
$$

where underlines denote all possible permutations. The bosonic states lie in the integer lattice of $\mathrm{SO}(8)$, they transform as a vector. The fermions have right-moving momenta with half-integer entries, they transform in the spinor representation $\mathbf{8}_{s}$. The oscillator excitations transform like the coordinates $Z^{i}, Z^{* i}$,

$$
\tilde{\alpha}_{-1} \equiv\left(\tilde{\alpha}_{-1}^{1}, \ldots, \tilde{\alpha}_{-1}^{4}, \tilde{\alpha}_{-1}^{* 1}, \ldots, \tilde{\alpha}_{-1}^{* 4}\right)=\mathbf{8}_{v} \text { of } \mathrm{SO}(8) .
$$

Hence the uncharged solutions (81) of the untwisted mass equations can be identified with the ten-dimensional supergravity multiplet:

$$
8_{v} \times 8_{v}=\underbrace{35}_{g_{M N}}+\underbrace{28}_{B_{M N}}+\underbrace{1}_{\phi}, \quad 8_{s} \times 8_{v}=\underbrace{56_{c}}_{\tilde{g}_{M}}+\underbrace{8_{c}}_{\tilde{\phi}} .
$$

Here $g_{M N}\left(\tilde{g}_{M}\right)$ denotes the graviton (gravitino), $\phi(\tilde{\phi})$ the dilaton (dilatino) and $B_{M N}$ the antisymmetric two-form in ten dimensions.

The action of the space group on three of the four complex coordinates breaks the $\mathrm{SO}(8)$ to the point group $\mathbb{Z}_{N} \subset \mathrm{SO}(6) \subset \mathrm{SO}(8)$ and four dimensional helicity $\mathrm{U}(1)_{h} \sim$ $\mathrm{SO}(2) \subset \mathrm{SO}(8)$,

$$
\mathrm{SO}(8) \rightarrow \mathbb{Z}_{N} \times \mathrm{U}(1)_{h} \subset \mathrm{SO}(6) \times \mathrm{U}(1)_{h}
$$

In our conventions the fourth entry of the vector $\mathbf{q}$ determines the transformation properties of a zero mode state under helicity $\mathrm{U}(1)_{h}$. Furthermore, we choose $q^{4}=0$ and $q^{4}=-1 / 2$ $\left(q^{4}=+1 / 2\right)$ to correspond to a scalar and a left-handed (right-handed) Weyl fermion in four dimensions, respectively.

Thus without the orbifold projections, the solutions (81) give rise to four gravitinos, implying $\mathcal{N}=4$ supersymmetry in four dimensions. The requirement that this should be broken to $\mathcal{N}=1$ results in constraints on the choice of viable twist vectors $\mathbf{v}$. First, we note that also in four dimensions gravitinos involve a vector and a spinor index. In (81) the spinor arises from the right-moving momentum and the vector from oscillators in flat space directions, $i=4$, which do not transform under the twist. Thus the orbifold condition (74) becomes

$$
\mathbf{q} \cdot \mathbf{v}=0 \bmod 1
$$


If we choose the vectors $\mathbf{q}= \pm(1 / 2,1 / 2,1 / 2,1 / 2)$ from the possibilities in (82) as representatives for the two helicity states of the four-dimensional gravitino, we recover the condition (12) for $\mathcal{N}=1$ supersymmetry:

$$
\sum_{j} v^{j}=0, \quad \quad v^{j} \neq 0 \bmod 1, \quad j=1,2,3 .
$$

In summary, the four-dimensional supergravity multiplet is given by the states (81), with $\mathbf{q}=(0,0,0, \pm 1), \pm(1 / 2,1 / 2,1 / 2,1 / 2)$ and oscillator excitations in flat space directions, $i=4$.

Further zero modes arise from excitations in internal directions, $i=1,2,3$. They are again subject to the orbifold projection conditions, and the number of solutions depends on the space group $\mathbb{Z}_{N}$. These states are 'geometrical moduli', they describe fluctuations of the internal metric around the fixed background.

\section{The gauge sector}

Further solutions of the mass equations $(79),(80)$ in the untwisted sector are $(I=1, \ldots, 16)$

$$
|\mathbf{q}\rangle_{R} \otimes \tilde{\alpha}_{-1}^{I}|0\rangle_{L}, \quad|\mathbf{q}\rangle_{R} \otimes|\mathbf{p}\rangle_{L},
$$

with $\mathbf{q}$ given by (82), and $\mathbf{p}^{2}=2$. The latter equation has 480 solutions, furthermore the oscillators above give rise to 16 uncharged Cartan generators. In total, (88) forms the gauge vector multiplet in the adjoint representation of $\mathrm{E}_{8} \times \mathrm{E}_{8}$ in ten dimensions.

Application of the orbifold projection conditions (74) restricts the allowed combinations of internal momenta $\mathbf{q}$ and $\mathbf{p}$ above. This never influences the number of Cartan generators, and therefore the rank of the gauge group cannot be reduced by orbifolding. However, some of the states with $q^{4}= \pm 1$, which correspond to vectors in four dimensions, are projected out and thus the local gauge symmetry of the effective theory is reduced to a subgroup of $\mathrm{E}_{8} \times \mathrm{E}_{8}$.

\section{The matter sector}

Matter arises from all twisted sectors $\mathcal{H}_{[g]}$, and also from the untwisted sector. It is given by all charged massless states which fulfill the orbifold projection conditions and transform as chiral multiplets from the four-dimensional perspective $\left(q^{4}=0, q^{4}=-1 / 2\right)$, or as their conjugates. The matter spectrum is very model dependent; we will discuss a specific example in detail in the following chapter.

\subsection{Interactions}

For realistic model building not only the spectrum is important, but also interactions between the various zero modes. They arise from correlation functions of string vertex 
operators, which can in principle be calculated for orbifolds with conformal field theory methods [11,61]. The results show that a superpotential coupling of the form

$$
W=\alpha \phi_{1} \cdots \phi_{M}
$$

where $\alpha$ is a moduli dependent constant and $\phi_{n}, n=1, \ldots, M$, are chiral multiplets of the low-energy effective theory, can only be present if the associated quantum numbers fulfill a certain set of conditions, the 'string selection rules'. Some of them have straightforward interpretations in the effective field theory as gauge invariance and $R$-charge conservation. Additionally, it is required that the string boundary conditions are such that they are consistent with string interaction diagrams. The resulting constraint is often referred to as the 'space group selection rule', it is sensitive to the localization properties of the involved states.

\subsubsection{Gauge invariance and discrete $R$-symmetry}

Consider a term of $M$ fields, which arises from a superpotential contribution (89) and couples two fermions to $M-2$ bosons. The explicit form of the string vertex operators for such an interaction then implies the following rules for the bosonic components of the chiral multiplets $\phi_{n}$ :

$$
\begin{aligned}
& \sum_{n=1}^{M} \mathbf{p}_{\mathrm{sh}}^{(n)}=0, \\
& \sum_{n=1}^{M} R_{(n)}^{j}=-1 \bmod N_{\left(v^{j}\right)}, \quad j=1,2,3 .
\end{aligned}
$$

Here the contributions from the multiplet $\phi_{n}$ are labeled by $(n)$, and $N_{\left(v^{j}\right)}$ is the order of the sub-twist $\vartheta_{(j)}$ in the plane $j$.

Equation (90) states gauge invariance of the superpotential. Similarly, Equation (91) describes charge conservation of three discrete $R$-symmetries, acting as ${ }^{9}$

$$
\phi(x, \theta) \stackrel{R^{j}}{\longmapsto} e^{2 \pi i R^{j} v^{j}} \phi\left(x, e^{\pi i v^{j}} \theta\right), \quad j=1,2,3,
$$

on chiral multiplets $\phi(x, \theta)$, where the $R^{j}$ are the bosonic $R$-charges that appear in (91), and $\theta$ denotes the Grassmannian coordinates of four-dimensional superspace, transforming as $\theta \mapsto e^{\pi i v^{j}} \theta$. The rule (91) can then be understood as

$$
W \stackrel{R^{j}}{\longmapsto} e^{2 \pi i \sum_{n} R_{(n)}^{j} v^{j}} W=e^{-2 \pi i v^{j}} W, \quad j=1,2,3,
$$

whence the action $\int \mathrm{d}^{2} \theta W$ is invariant under the three discrete $R$-transformations. Note that there is no continuous $R$-symmetry in the effective theory, as it is often assumed in field theoretical model building.

\footnotetext{
${ }^{9}$ Here no summation over $j$ is implied.
} 
As apparent from (92), the discrete $R$-symmetries do not commute with supersymmetry. This can also be seen from their definition (61),

$$
R^{j} \equiv q_{\mathrm{sh}}^{i}+\tilde{N}^{* i}-\tilde{N}^{i}=k v^{j} \bmod 1, \quad j=1,2,3,
$$

which implies a distinction between bosons and fermions in a multiplet. Note that these $R$-charges also appear in the transformation phase (73).

The $N_{\left(v^{j}\right)}$-fold application of the transformation (93) together with (94) leads to a condition on the twist quantum numbers $k_{(n)}$ :

$$
e^{2 \pi i c^{j} \sum_{n} k_{(n)} / N_{\left(v^{j}\right)}}=1, \quad \quad c^{j} \equiv N_{\left(v^{j}\right)} v^{j} \in \mathbb{Z}, \quad j=1,2,3 .
$$

Since the appearing integers $c^{j}$ cannot be divisors of $N_{\left(v^{j}\right)}$, this implies the rule

$$
\sum_{n=1}^{M} k_{(n)}=0 \bmod N
$$

where $N$ is the order of the orbifold twist. The fact that this well-known sum rule is related to the $R$-symmetries was first noted in [17].

\subsubsection{Space group selection rule}

A string interaction is only possible if the boundary conditions $\left[g_{n}\right]=\left[\left(\theta^{k_{(n)}}, m_{a}^{(n)} \mathbf{e}_{a}\right)\right]$ associated to the $\phi_{n}$ in (89) multiply to the identity:

$$
\prod_{n=1}^{M}\left[g_{n}\right]=(\mathbb{1}, 0) .
$$

The conjugacy classes (21) can be expressed as

$$
\left[g_{n}\right]=\left(\theta^{k_{(n)}}, \Theta m_{a}^{(n)} \mathbf{e}_{a}+\Lambda_{k_{(n)}}\right),
$$

with $\Theta=\left\{\theta^{q} \mid q \in \mathbb{Z}\right\}$ and sub-lattices $\Lambda_{k_{(n)}}=\left(\mathbb{1}-\theta^{k_{(n)}}\right) \Lambda$, which were introduced in (22). This gives

$$
\left[g_{n}\right]\left[g_{m}\right]=\left(\theta^{k_{(n)}+k_{(m)}}, \Theta\left(m_{a}^{(n)} \mathbf{e}_{a}+m_{a}^{(m)} \mathbf{e}_{a}\right)+\Lambda_{k_{(n)}+k_{(m)}}\right),
$$

from which we infer that (97) again gives the condition (96), and additionally ${ }^{10}$

$$
\sum_{n=1}^{M} m_{a}^{(n)} \mathbf{e}_{a}=0 \bmod \sum_{n=1}^{M} \Lambda_{k_{(n)}} .
$$

This condition restricts the allowed contributions from different fixed points to a superpotential coupling. It can thus be interpreted as a constraint which is sensitive to localization properties of the involved states on the orbifold geometry.

\footnotetext{
${ }^{10}$ This follows from $\Lambda_{\sum k_{(n)}}=0$ and invariance of (98) under $m_{a}^{(n)} \mathbf{e}_{a} \rightarrow m_{a}^{(n)} \mathbf{e}_{a}+\lambda$ for $\lambda \in \Lambda_{k_{(n)}}$.
} 


\section{A local GUT in six dimensions}

The phenomenological success of orbifold GUTs in five [31,32] or six [33,34] dimensions was one of the main motivations to reconsider orbifold compactifications of the heterotic string [11-13], twenty years after their first proposal [30]. Here an effective orbifold GUT of co-dimension two is described, which is associated with an anisotropic orbifold compactification of the heterotic string [16].

The model which is presented here has the same geometry and gauge embedding as the model [13]. The latter is an example for an effective MSSM-like model in four dimensions, which follows from compactifying the six internal dimensions of heterotic string theory on a specific orbifold, with specific Wilson lines. It is a representative of a full class of models, as discussed in [14], its spectrum is reviewed in Appendix E. Here we focus on the possibility that such an orbifold compactification and the idea of supersymmetric grand unification in the extra dimensions can be unified. This may be achieved by assuming that one or two of the internal radii are considerably larger than the others, yielding an anisotropic compactification [42]. For heterotic orbifolds this is an attractive possibility to incorporate higher-dimensional GUTs as an intermediate step of the compactification [11-13,43]. In this picture, the GUT scale is linked to the compactification scale of some large internal compact dimensions. At the string scale, there is no effective field theory limit and full string propagation on the ten-dimensional geometry has to be considered.

In fact, there is some tension between the requirement that weakly coupled heterotic string theory is applicable and precision gauge coupling unification [43]. Here we shall assume that consistency with this bound can be achieved in the present model ${ }^{11}$, once the vacuum structure and the stabilization mechanism are fully understood.

Apart from the phenomenological interest in the realization of a supersymmetric GUT in higher dimensions within the framework of string theory, the main benefit of our approach is a better understanding of the structure of the model. This will then lead to a simplification of the discussion of the vacuum (cf. Chapter 4), and it will provide a link towards smooth Calabi-Yau compactifications without explicit blow-ups (cf. Chapter 5).

\subsection{The $\mathbb{Z}_{6-\text { II }}$ orbifold}

We consider the compactification of six dimensions on the orbifold

$$
\mathcal{M}_{6}=\frac{\mathbb{C}^{3}}{\Gamma_{\mathbb{Z}_{6-\mathrm{II}}} \times \mathbb{Z}_{6-\mathrm{II}}} \equiv \frac{\mathbb{C} / \Gamma_{\mathrm{G}_{2}} \times \mathbb{C} / \Gamma_{\mathrm{SU}(3)} \times \mathbb{C} / \Gamma_{\mathrm{SO}(4)}}{\mathbb{Z}_{6-\mathrm{II}}},
$$

where $\Gamma_{G}, G=\mathrm{G}_{2}, \mathrm{SU}(3), \mathrm{SO}(4)$, denotes the two-dimensional root lattice ${ }^{12}$ of the Lie group $G$ and we omit factors of $\pi$. The action of the point group $\mathbb{Z}_{6-\text { II }}$ is defined by the

\footnotetext{
${ }^{11}$ For related work on this issue see [44].

${ }^{12}$ Throughout the work we will frequently use the term ' $G$-plane', either referring to the two-torus $\mathbb{C} / \Gamma_{G}$ or the corresponding non-compact plane $\mathbb{C}$, depending on the context.
} 


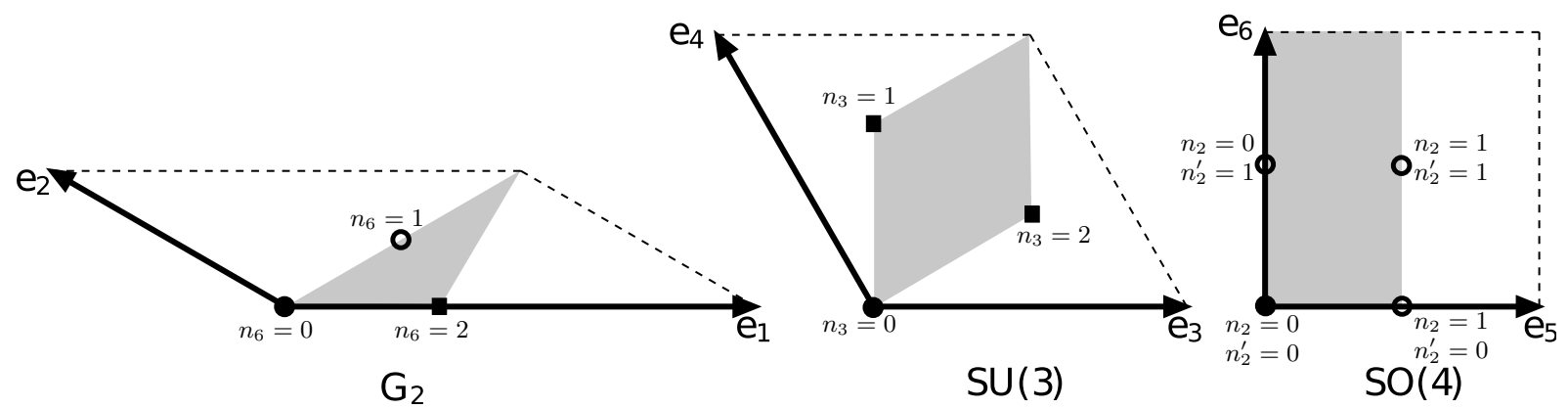

Figure 2: The $\mathbb{Z}_{6-\text { II }}$ orbifold geometry. The shaded area is the fundamental domain of the orbifold, which by definition is invariant under the twist (102). Note that in each plane the geometry is that of the surface of a pillow. Its boundary points are singularities, marked by circles, boxes and bold dots, denoting fixed points with respect to a space group element with $k=3,2,1$, respectively. The fact that for $k=1$ the fixed points in the SU(3)- and the $\mathrm{SO}(4)$-plane are also invariant is not illustrated. The explicit coordinates and space group elements are collected in Table 2.

\begin{tabular}{l|l||l|l||l|l} 
Plane & Lattice vector & Plane & Lattice vector & Plane & Lattice vector \\
\hline$G_{2}$ & $\mathbf{e}_{1}=(1,0,0 ; 0)$ & $\mathrm{SU}(3)$ & $\mathbf{e}_{3}=(0,1,0 ; 0)$ & $\mathrm{SO}(4)$ & $\mathbf{e}_{5}=(0,0,1 ; 0)$ \\
& $\mathbf{e}_{2}=\frac{1}{\sqrt{3}}\left(e^{\frac{5 \pi}{6} i}, 0,0 ; 0\right)$ & & $\mathbf{e}_{4}=\left(0, e^{\frac{2 \pi}{3}}, 0 ; 0\right)$ & & $\mathbf{e}_{6}=(0,0, i ; 0)$
\end{tabular}

Table 1: Definition of the root lattice $\Gamma_{\mathbb{Z}_{6-\mathrm{II}}}$.

twist vector

$$
\mathbf{v}_{6}=\left(-\frac{1}{6},-\frac{1}{3}, \frac{1}{2} ; 0\right)
$$

This orbifold was first suggested in [11] and became a widely studied candidate for realistic model building [12-14,49,62]. Its geometry is summarized in Figure 2, the explicit lattice vectors are given in Table 1.

The most apparent property of the $\mathbb{Z}_{6-\text { II }}$ orbifold is the presence of invariant tori under the sub-symmetries $\mathbb{Z}_{2}$ and $\mathbb{Z}_{3}$, generated by

$$
\begin{array}{ll}
\mathbb{Z}_{2}: & \mathbf{v}_{2} \equiv 3 \mathbf{v}_{6}=\left(-\frac{1}{2},-1, \frac{3}{2} ; 0\right), \\
\mathbb{Z}_{3}: & \mathbf{v}_{3} \equiv 2 \mathbf{v}_{6}=\left(-\frac{1}{3},-\frac{2}{3}, 1 ; 0\right) .
\end{array}
$$

This shows that the $\mathrm{SU}(3)$ - and the $\mathrm{SO}(4)$-plane are invariant under the $\mathbb{Z}_{2}$ and the $\mathbb{Z}_{3}$ sub-twists, respectively. Consequently, states which are localized in the $\mathrm{SU}(3)$-plane are bulk states in the $\mathrm{SO}(4)$-plane, and vice-versa. 


\begin{tabular}{|c|c|c|c|c|c|c|}
\hline \multirow[t]{2}{*}{ Plane } & \multicolumn{2}{|c|}{ Fixed Point } & \multicolumn{2}{|c|}{ Constructing element $g$} & \multicolumn{2}{|c|}{$\gamma$-phase } \\
\hline & Label & Coordinate & $k$ & $m_{a} \mathbf{e}_{a}$ & $N_{(g)}$ & $\gamma$ \\
\hline \multirow[t]{4}{*}{$G_{2}$} & $n_{6}=0$ & 0 & $1,2,3,4,5$ & 0 & 1 & 0 \\
\hline & $n_{6}=1$ & $\frac{1}{2} \pi \mathbf{e}_{1}+\frac{1}{2} \pi \mathbf{e}_{2}$ & 3 & \multirow[t]{2}{*}{$\mathbf{e}_{1}+\mathbf{e}_{2}$} & 3 & $\frac{1}{3}, \frac{2}{3}, 1$ \\
\hline & \multirow[t]{2}{*}{$n_{6}=2$} & \multirow[t]{2}{*}{$\frac{1}{3} \pi \mathbf{e}_{1}$} & 2 & & \multirow[t]{2}{*}{2} & \multirow[t]{2}{*}{$\frac{1}{2}, 1$} \\
\hline & & & 4 & $-\mathbf{e}_{2}$ & & \\
\hline \multirow[t]{5}{*}{$\mathrm{SU}(3)$} & $n_{3}=0$ & 0 & $1,2,4,5$ & 0 & & \\
\hline & \multirow[t]{2}{*}{$n_{3}=1$} & \multirow[t]{2}{*}{$\frac{1}{3} \pi \mathbf{e}_{3}+\frac{2}{3} \pi \mathbf{e}_{4}$} & 1,4 & $\mathbf{e}_{4}$ & & \\
\hline & & & 2,5 & \multirow[t]{2}{*}{$\mathbf{e}_{3}+\mathbf{e}_{4}$} & & \\
\hline & \multirow[t]{2}{*}{$n_{3}=2$} & \multirow[t]{2}{*}{$\frac{2}{3} \pi \mathbf{e}_{3}+\frac{1}{3} \pi \mathbf{e}_{4}$} & 1,4 & & & \\
\hline & & & 2,5 & $\mathbf{e}_{3}$ & & \\
\hline \multirow[t]{4}{*}{$\mathrm{SO}(4)$} & $\left(n_{2}, n_{2}^{\prime}\right)=(0,0)$ & 0 & $1,3,5$ & 0 & & \\
\hline & $\left(n_{2}, n_{2}^{\prime}\right)=(1,0)$ & $\frac{1}{2} \pi \mathbf{e}_{5}$ & $1,3,5$ & $\mathbf{e}_{5}$ & & \\
\hline & $\left(n_{2}, n_{2}^{\prime}\right)=(0,1)$ & $\frac{1}{2} \pi \mathbf{e}_{6}$ & $1,3,5$ & $\mathbf{e}_{6}$ & & \\
\hline & $\left(n_{2}, n_{2}^{\prime}\right)=(1,1)$ & $\frac{1}{2} \pi \mathbf{e}_{5}+\frac{1}{2} \pi \mathbf{e}_{6}$ & $1,3,5$ & $\mathbf{e}_{5}+\mathbf{e}_{6}$ & & \\
\hline
\end{tabular}

Table 2: List of the fixed points of the $\mathbb{Z}_{6-\text { II }}$ orbifold. Note that the given coordinates and the translations of the constructing elements only refer to the given plane and have to be superposed. The planes $\mathrm{SU}(3)$ and $\mathrm{SO}(4)$ are invariant under the $\mathbb{Z}_{2}$ and $\mathbb{Z}_{3}$ subtwists which are generated by $\theta^{3}$ and $\theta^{2}$, respectively. This leads to the appearance of fixed planes, expressed by arbitrary coordinates in the invariant plane. The $\gamma$-phase is entirely determined by the localization in the $\mathrm{G}_{2}$-plane. The $\gamma^{\prime}$-phase is universally given by $\gamma^{\prime}=$ $\frac{1}{6}\left(0,0,2 l_{3}, 2 l_{3}, 3 l_{5}, 3 l_{6}\right)$, for integers $l_{3}, l_{5}, l_{6}$.

\section{Fixed point structure}

The geometry has 12 fixed points and 14 fixed tori, related to 44 sectors $\mathcal{H}_{[g]}$. Each of them corresponds to one of the twisted sectors $T_{k}$ and is specified by the following labels:

12 fixed points in $T_{1}: \quad n_{6}=0, \quad n_{3}=0,1,2, \quad n_{2}=0,1, \quad n_{2}^{\prime}=0,1$,

6 fixed tori in $T_{2}: \quad n_{6}=0,2, \quad n_{3}=0,1,2$,

8 fixed tori in $T_{3}: \quad n_{6}=0,1, \quad n_{2}=0,1, \quad n_{2}^{\prime}=0,1$,

6 fixed tori in $T_{4}: \quad n_{6}=0,2, \quad n_{3}=0,1,2$,

12 fixed points in $T_{5}: \quad n_{6}=0, \quad n_{3}=0,1,2, \quad n_{2}=0,1, \quad n_{2}^{\prime}=0,1$.

The localization of these fixed points is depicted in Figure 2 and quantified in Table 2, from which also the associated space group elements can be inferred.

The number of different sectors coincides with the number of disjoint conjugacy classes of the space group ${ }^{13}$. This follows from the observation that the conjugacy class associated with an element $\left(\theta^{k}, m_{a} \mathbf{e}_{a}\right)$ can be written as the product of all twists acting on the

\footnotetext{
${ }^{13}$ We are grateful to Christoph Lüdeling for pointing this out.
} 
translation, and the conjugacy class of the twist,

$$
\left[\left(\theta^{k}, m_{a} \mathbf{e}_{\mathbf{a}}\right)\right]=\left(\mathbb{1}, \Theta m_{a} \mathbf{e}_{a}\right)\left(\theta^{k}, \Lambda_{k}\right) .
$$

Here $\Theta=\left\{\theta^{\tilde{k}} \mid \tilde{k}=0, \ldots, 5\right\}$ collects all possible twists and $\Lambda_{k}$ denotes the $k$-th sub-lattice, defined in (22) and explicitly given in Table 3. For each $k$, one can count the number of translations $m_{a} \mathbf{e}_{a}$ that induce inequivalent sets and recover the above numbers.

Note that both the $\mathbb{Z}_{2}$ and the $\mathbb{Z}_{3}$ sub-twist act non-trivially on the $\mathrm{G}_{2}$-plane. Fixed points of the covering space which are invariant under only one of these twists are then mapped onto conjugated fixed points by the other, cf. (24), (64). Since the string mode expansions, which constitute the starting point of quantization, are formulated in the covering space, conjugated fixed points give rise to different Hilbert spaces. However, on the orbifold the two points agree and the Hilbert spaces have to be united, as described in Section 2.3. Physical states are then superpositions of states at the different conjugated fixed points. They are specified by quantum numbers $\gamma^{\prime}, \gamma$ and take the form (68).

We illustrate this with the example of the non-trivial $\mathbb{Z}_{3}$ fixed point in the $\mathrm{G}_{2}$-plane, labeled by $n_{6}=2$. Table 2 specifies the generating space group element $g$ and the coordinate $z_{g}^{1}$ as

$$
g=\left(\theta^{2}, \mathbf{e}_{1}+\mathbf{e}_{2}\right), \quad z_{g}^{1}=\frac{1}{3} \pi e_{1}^{1},
$$

where $e_{a}^{i}$ denotes the $i$-th entry of the vector $\mathbf{e}_{a}$ from Table 1 . The action of $h=\theta^{3}$ is then

$$
z_{g}^{1} \stackrel{h}{\mapsto} \tilde{z}^{1}=\vartheta_{(1)}^{3} z_{g}^{1}=-z_{g}^{1},
$$

which does not lie inside the chosen fundamental domain of the orbifold. This coordinate is the fixed point coordinate of the conjugated element $h g h^{-1}$ :

$$
h g h^{-1}=\left(\theta^{2},-\mathbf{e}_{1}-\mathbf{e}_{2}\right), \quad \tilde{z}^{1} \stackrel{h g h^{-1}}{\longmapsto}-\left(\vartheta_{(1)}^{2} z_{g}^{1}+e_{1}^{1}+e_{2}^{1}\right)=-z_{g}^{1}=\tilde{z}^{1} .
$$

Physical states are superpositions

$$
n_{6}=2: \quad|\gamma\rangle=\left|z_{g}^{1}\right\rangle+e^{-2 \pi i \gamma}\left|\tilde{z}^{1}\right\rangle, \quad \gamma=\gamma_{1,2}\left(\theta^{3}\right)=\frac{1}{2}, 1
$$

up to normalization. They are eigenstates of $h$,

$$
|\gamma\rangle \stackrel{h}{\mapsto}\left|\tilde{z}^{1}\right\rangle+e^{-2 \pi i \gamma}\left|z_{g}^{1}\right\rangle=e^{2 \pi i \gamma}|\gamma\rangle,
$$

as discussed in (68). Similarly the $\mathbb{Z}_{2}$ fixed point gives three eigenstates, with eigenvalues

$$
n_{6}=1: \quad \gamma=\gamma_{1,2,3}\left(\theta^{2}\right)=\frac{1}{3}, \frac{2}{3}, 1 .
$$

In summary, states with the same quantum number $n_{6}$ are localized at the same point in the $\mathrm{G}_{2}$-plane of the orbifold. Furthermore, they are grouped into two or three sub-classes 
of $\mathbb{Z}_{6}$ eigenstates, specified by two or three possible eigenvalues $\gamma_{j}\left(\theta^{\tilde{k}}\right)$ in (109) or (111), respectively.

The translational $\gamma^{\prime}$-phases under a transformation by $h=\left(\theta^{\tilde{k}}, \tilde{m}_{a} \mathbf{e}_{a}\right)$ for the $\mathbb{Z}_{6-\mathrm{II}}$ geometry are calculated in Appendix A.3, with result

$$
\gamma^{\prime}=\left(0,0, \frac{l_{3}}{3}, \frac{l_{3}}{3}, \frac{l_{5}}{2}, \frac{l_{6}}{2}\right)
$$

where $l_{3}, l_{5}, l_{6}$ are independent integers. This directly corresponds to the order of the lattice vector $\mathbf{e}_{a}$. For directions which are related by symmetry transformations the entries coincide, in the case of unit order of a lattice direction they have to be trivial.

Recall that $\gamma^{\prime}$ and $\gamma$ describe superpositions of states at fixed points which are related to the constructing element $g=\left(\theta^{k}, m_{a} \mathbf{e}_{a}\right)$ by translational and rotational conjugation, respectively, as apparent from (68). The phase which arises upon application of $h$ was given in (69),

$$
\tilde{\Phi}_{h} \equiv e^{2 \pi i \gamma_{a}^{\prime}\left(\tilde{k} m_{a}-k \tilde{m}_{a}\right)} e^{2 \pi i \gamma \tilde{k}}
$$

For elements $h$ which commute with $g$ one has

$$
\gamma \tilde{k}=0 \bmod 1, \quad \tilde{k} \gamma_{a}^{\prime} m_{a}=k \gamma_{a}^{\prime} \tilde{m}_{a} \bmod 1,
$$

such that the states $\left|\gamma^{\prime}, \gamma\right\rangle_{[g]}$ have the property

$$
[h, g]=0: \quad \tilde{\Phi}_{h}=1 .
$$

Also note that sub-lattice translations $\tilde{m}_{a} \mathbf{e}_{a} \in \Lambda_{\tilde{k}}$ from Table 3 do not contribute to the phase, $\gamma_{a}^{\prime} \tilde{m}_{a}=0 \bmod 1$. This is an important property, since it has the consequence that it is sufficient to evaluate the consistency condition for finitely many elements $h \in S$, which are representatives of disjoint conjugacy classes.

As an example for the translational superposition, consider the fixed point at the origin of the $\mathrm{SO}(4)$-plane. Its constructing element $(\theta, 0)$ does not commute with lattice translations in that plane, for example

$$
\left(\mathbb{1}, \mathbf{e}_{5}\right)\left|\mathbf{z}_{(\theta, 0)}\right\rangle=\left|\mathbf{z}_{\left(\theta, 2 \mathbf{e}_{5}\right)}\right\rangle .
$$

The eigenstates of translations in the direction $\mathbf{e}_{5}$ are then given by the superpositions

$$
\left|\gamma_{5}^{\prime}\right\rangle_{[(\theta, 0)]}=\sum_{m \in \mathbb{Z}} e^{-2 \pi i \gamma_{5}^{\prime} m}\left|\mathbf{z}_{\left(\theta, 2 m \mathbf{e}_{5}\right)}\right\rangle, \quad \quad \gamma_{5}^{\prime}=\frac{1}{2}, 1
$$

with eigenvalues $\mp 1$. 


\subsubsection{The gauge embedding}

For the gauge embedding (38) of the space group $S$ into the gauge group $\mathrm{E}_{8} \times \mathrm{E}_{8}$, the independent Wilson lines have to be identified. They correspond to the inequivalent lattice shifts contained in $S$, which can be read off from the $\gamma_{a}^{\prime}$ values in (112). There are no Wilson lines in the $\mathrm{G}_{2}$-plane, one of order three in the $\mathrm{SU}(3)$-plane, and two of order two in the $\mathrm{SO}(4)$-plane. We denote them by

$$
\mathbf{W}_{(3)} \equiv \mathbf{W}_{3}, \quad \mathbf{W}_{(2)} \equiv \mathbf{W}_{5}, \quad \mathbf{W}_{(2)}^{\prime} \equiv \mathbf{W}_{6},
$$

where the $\mathbf{W}_{a}$ are associated to the lattice vector $\mathbf{e}_{a}$, cf. (38). The new subscripts $(n)$ correspond to the order $n$ of the Wilson line.

Following [13], we choose the gauge embedding

$$
\begin{aligned}
\mathbf{V}_{6} & =\left(-\frac{1}{2},-\frac{1}{2}, \frac{1}{3}, 0,0,0,0,0\right)\left(\frac{17}{6},-\frac{5}{2},-\frac{5}{2},-\frac{5}{2},-\frac{5}{2},-\frac{5}{2},-\frac{5}{2}, \frac{5}{2}\right) \\
\mathbf{W}_{(2)} & =\left(-\frac{1}{2}, 0,-\frac{1}{2}, \frac{1}{2}, \frac{1}{2}, 0,0,0\right)\left(\frac{23}{4},-\frac{25}{4},-\frac{21}{4},-\frac{19}{4},-\frac{25}{4},-\frac{21}{4},-\frac{17}{4}, \frac{17}{4}\right), \\
\mathbf{W}_{(2)}^{\prime} & =0 \\
\mathbf{W}_{(3)} & =\left(-\frac{1}{6}, \frac{1}{2}, \frac{1}{2},-\frac{1}{6},-\frac{1}{6},-\frac{1}{6},-\frac{1}{6},-\frac{1}{6}\right)\left(0,-\frac{2}{3}, \frac{1}{3}, \frac{4}{3},-1,0,0,0\right) .
\end{aligned}
$$

With this choice the strong modular invariance conditions (42) are fulfilled.

The local shift vectors (57) for a fixed point element $g$ with quantum numbers $k, n_{2}$ and $n_{3}$ can now be written in an economical form,

$$
\mathbf{V}_{g} \equiv \mathbf{V}_{\left(k, n_{2}, n_{3}\right)}=k\left(\mathbf{V}_{6}+n_{2} \mathbf{W}_{(2)}+n_{3} \mathbf{W}_{(3)}\right)
$$

If $g$ corresponds to a fixed $\mathrm{SU}(3)$ - or $\mathrm{SO}(4)$-torus, the above formula applies with $n_{3}=0$ or $n_{2}=0$, respectively.

The definition of the gauge embedding is the final input which is necessary for the calculation of the spectrum. In summary, the model is specified by the tori lattices (Table 1), from which the twist vector (102) can be inferred, and the gauge embedding (119).

\subsubsection{Space group selection rules for interactions}

The string selection rules for allowed superpotential couplings in the general case were discussed in section 2.4. While some of them have straightforward interpretations in the effective field theory as gauge invariance and $R$-symmetry, this is not so clear for the space group selection rule (100). For a coupling of $M$ chiral multiplets,

$$
W=\alpha \phi_{1} \cdots \phi_{M}
$$

with quantum numbers labeled by the index $(l)$, it reads

$$
\sum_{l} m_{a}^{(l)} \mathbf{e}_{a}=0 \bmod \sum_{l} \Lambda_{k_{(l)}}
$$




\begin{tabular}{l|l|l|l} 
Sub-lattice & $G_{2}$-plane & $\mathrm{SU}(3)$-plane & $\mathrm{SO}(4)$-plane \\
\hline$\Lambda_{1}=\left(\mathbb{1}-\theta^{1}\right) \Lambda$ & $a \mathbf{e}_{1}+b \mathbf{e}_{2}$ & $a \mathbf{e}_{3}+(3 b-a) \mathbf{e}_{4}$ & $2 a \mathbf{e}_{5}+2 b \mathbf{e}_{6}$ \\
$\Lambda_{2}=\left(\mathbb{1}-\theta^{2}\right) \Lambda$ & $a \mathbf{e}_{1}+3 b \mathbf{e}_{2}$ & $a \mathbf{e}_{3}+(3 b-a) \mathbf{e}_{4}$ & 0 \\
$\Lambda_{3}=\left(\mathbb{1}-\theta^{3}\right) \Lambda$ & $2 a \mathbf{e}_{1}+2 b \mathbf{e}_{2}$ & 0 & $2 a \mathbf{e}_{5}+2 b \mathbf{e}_{6}$
\end{tabular}

Table 3: The sub-lattices of the $\mathbb{Z}_{6-\text { II }}$ orbifold. $a$ and $b$ are independent integers in each plane. Note that $\Lambda_{6-k}=\Lambda_{k}$.

The sub-lattices that appear on the right hand side of this equation are listed in Table 3 for the $\mathbb{Z}_{6-\text { II }}$ geometry. The left hand side is determined by the number of states at a fixed point $g$, which can be represented by labels $\left(k, n_{2}, n_{2}^{\prime}, n_{3}, n_{6}\right)$, cf. (104).

For the $\mathrm{G}_{2}$-plane, the sub-lattices $\Lambda_{k}$ do not coincide for all choices of $k$, cf. Table 3 . We thus consider each of them separately. For $k=1,5$, the sub-lattice $\Lambda_{k}=\Lambda_{1}$ and the root lattice of $G_{2}$ are identical. Thus in that case there is no restriction from space group selection rules; Equation (122) is always fulfilled.

If all states of a coupling are in the $T_{2}$ or $T_{4}$ sector, only the sub-lattice $\Lambda_{2}$ appears, which implies a non-trivial condition for the allowed combinations of $m_{a}^{(l)}$. Let $N_{k, n_{6}}$ denote the number of states that contribute to the coupling (121) and which are localized at a fixed point of the $\mathrm{G}_{2}$-plane with the corresponding quantum numbers. Inspection of Table 2 then gives

$$
\sum_{l} m_{a}^{(l)} \mathbf{e}_{a}=N_{2,2} \mathbf{e}_{1}+\left(N_{2,2}-N_{4,2}\right) \mathbf{e}_{2}
$$

This vector lies in $\left\{a \mathbf{e}_{1}+3 b \mathbf{e}_{2} \mid a, b \in \mathbb{Z}\right\} \subset \Lambda_{2}$ if

$$
N_{2,2}+2 N_{4,2}=0 \bmod 3 .
$$

Similarly one can evaluate (122) for the case that all contributions to a coupling come from the $T_{3}$ sector,

$$
\sum_{l} m_{a}^{(l)} \mathbf{e}_{a}=N_{3,1}\left(\mathbf{e}_{1}+\mathbf{e}_{2}\right)
$$

which is in $\left\{2 a \mathbf{e}_{1}+2 b \mathbf{e}_{2} \mid a, b \in \mathbb{Z}\right\} \subset \Lambda_{3}$ for

$$
N_{3,1}=0 \bmod 2 .
$$

In terms of the quantum numbers of the individual states, (124) and (126) can be unified by the following rule:

$$
\sum_{l} k^{(l)} n_{6}^{(l)}=0 \bmod 6
$$

Note that states with $k=1,5$ give no contribution to the sum since they always have $n_{6}=0$. We stress that the rule (127) only applies to couplings which have the property 
that the contributing multiplets either all come from $T_{1}$ and $T_{5}$, or they all come from $T_{2}$ and $T_{4}$, or they all arise from $T_{3}$. Any term with contributions from at least two of these three branches will contain the sub-lattice $\Lambda_{1}$ in the space group selection rule (122), which completely covers the $G_{2}$ root lattice. In that case the rule (122) becomes trivial and (127) does not apply.

A similar analysis is performed in Appendix A.2 for the $\mathrm{SU}(3)$ - and the $\mathrm{SO}(4)$-plane. In summary, the space group selection rules for a coupling of $M$ multiplets are

$$
\begin{aligned}
G_{2}: \quad \sum_{l=1}^{M} k^{(l)} n_{6}^{(l)} & =0 \bmod 6, \quad \text { if }\left\{\begin{array}{c}
k^{(l)}-k^{\left(l^{\prime}\right)}=0 \bmod 6 \\
\text { or } \\
k^{(l)}+k^{\left(l^{\prime}\right)}=0 \bmod 6
\end{array}\right\} \text { for all } l, l^{\prime}, \\
\mathrm{SU}(3): \quad \sum_{l=1}^{M} k^{(l)} n_{3}^{(l)} & =0 \bmod 3, \\
\mathrm{SO}(4): \quad \sum_{l=1}^{M} k^{(l)} n_{2}^{(l)} & =0 \bmod 2, \\
\sum_{l=1}^{M} k^{(l)} n_{2}^{\prime(l)} & =0 \bmod 2 .
\end{aligned}
$$

These rules will be interpreted as discrete symmetries in the following chapter.

\subsection{The effective orbifold GUT}

Effective orbifold GUTs can be derived from the heterotic string by assuming that the six internal dimensions are compactified on an anisotropic geometry. For the $\mathbb{Z}_{6-\text { II }}$ orbifold this can be realized by taking some of the independent radii significantly larger than the others. We now present the specific example which will then be studied for the remainder of this paper. The main motivation for the work is to understand more of the mechanisms behind the zero mode spectra and couplings. If an intermediate effective GUT is realized in nature, the knowledge of the details of a particular model can help to identify generic features of such compactifications. The hope is that eventually these may be related to testable predictions. However, orbifold models appear to be rather ambiguous and first it is necessary to learn more about their structure. On a technical level, the intermediate GUT approach simplifies the discussion, since the local GUT model is equipped with a larger symmetry than the four-dimensional effective theory. We shall profit from that when we study the vacua of the model in the next chapter.

\subsubsection{Anisotropic limits of orbifolds}

The $\mathbb{Z}_{6-\text { II }}$ orbifold has four independent radii: One each for the $\mathrm{G}_{2}$-plane and the SU(3)plane, and another two for the $\mathrm{SO}(4)=\mathrm{SU}(2) \times \mathrm{SU}(2)$-plane. Thus in principle many effective GUT models can be derived from this geometry, with any dimension between five 
and ten. In each case the anisotropy allows one to split the geometry into a small and a large part (here embedded into six real dimensions),

$$
\mathbb{R}^{6} / \Gamma_{\mathbb{Z}_{6-\mathrm{II}}}=\underbrace{\mathbb{R}^{m} / \Gamma_{\text {small }}}_{T_{\text {small }}^{m}} \times \underbrace{\mathbb{R}^{n} / \Gamma_{\text {large }}}_{T_{\text {large }}^{n}}, \quad \quad \mathcal{M}_{\text {eff }}=T_{\text {large }}^{n} / \mathbb{Z}_{N_{\text {eff }}},
$$

with $m+n=6$. This leads to a specific spectrum, gauge group and number of supersymmetry generators for the effective field theory on the $\mathbb{Z}_{N_{\text {eff }}}$ orbifold $\mathcal{M}_{\text {eff }}$. The order $N_{\text {eff }}$ of the effective orbifold is given by the order of the $\mathbb{Z}_{6-\text { II }}$ twist in $T_{\text {large }}^{n}$. The full list of possibilities and the resulting bulk gauge groups is given in table D.13 of [13].

The massless spectrum of the string on a given compact orbifold geometry is independent of the size of the compactification. However, the masses of the Kaluza-Klein modes will depend on the moduli that describe the internal geometry. In the limit of infinite volume of $T_{\text {large }}^{n}$, some of these states become massless; they are zero modes of the compactification of the small dimensions in $T_{\text {small }}^{m}$, but not of the full geometry $\mathbb{R}^{6} / \Gamma_{\mathbb{Z}_{6-\text { II }}}$ at finite volume. In the effective intermediate model $\mathcal{M}_{\text {eff }}$, these states correspond to bulk states with odd boundary conditions, which are projected out in the low-energy limit.

We derive the effective orbifold model in a two-step procedure:

1. Treat $T_{\text {large }}^{n}$ effectively as flat space and compactify only the dimensions in $T_{\text {small }}^{m}$ on a compact geometry $\mathcal{M}_{\text {small }}$. In flat space all coordinates are independent, so the isometries of the latter coincide with the subset of the full space group $S$ which leaves $T_{\text {large }}^{n}$ invariant,

$$
S_{\text {small }}=\left\{g \in S \mid T_{\text {large }}^{n} \text { is invariant under } g\right\} .
$$

The resulting zero mode spectrum is the bulk spectrum of $\mathcal{M}_{\text {eff }}$.

2. For the local spectrum at a fixed point of $\mathcal{M}_{\text {eff }}$, related to a space group element $g_{f} \in S$, calculate the zero modes of the full $\mathbb{Z}_{6-\text { II }}$ compactification to four dimensions which correspond to such a localization. These states should also be present in the anisotropic limit, and no new states should arise. For bulk states of the effective field theory, the corresponding local phase $\Phi_{g_{f}}$ defines the behavior of the associated field under the local $\mathbb{Z}_{N_{\mathrm{eff}}}$ twist. Zero modes are then required to be compatible with all local boundary conditions, $\Phi_{g_{f}}=1$.

The latter requirement is indeed equivalent to the projection conditions in the case of a one-step compactification to four dimensions. This follows from the observation that arbitrary elements $h \in S$ can be decomposed into elements which represent the different twisted sectors. Each of them has a unique description in terms of the labels $k, n_{6}, n_{3}, n_{2}, n_{2}^{\prime}$, as given in (104), and corresponds to a generating space group element, which we choose as in Table 2 . With notation $g_{k, \mathbf{n}}$, where $\mathbf{n}=\left(n_{6}, n_{3}, n_{2}, n_{2}^{\prime}\right)$, one can write

$$
h=\prod_{f=(k, \mathbf{n})} g_{f}^{l_{f}}, \quad l_{f} \in \mathbb{Z},
$$


where $f=(k, \mathbf{n})$ runs over all 44 fixed point sectors. For a given $h \in S$ and the identification $g_{f}^{0} \equiv(\mathbb{1}, 0)$ it is always possible to find an appropriate set of exponents $l_{f}$. For example,

$$
\begin{array}{ll}
\mathbf{e}_{1}=g_{3,(1,0,0,0)} g_{3,(0,0,0,0)} g_{4,(2,0,0,0)} g_{2,(0,0,0,0)}, & \mathbf{e}_{2}=g_{3,(0,0,0,0)} g_{4,(2,0,0,0)} g_{5,(0,0,0,0)}, \\
\mathbf{e}_{3}=g_{2,(0,2,0,0)} g_{4,(0,0,0,0)}, & \mathbf{e}_{4}=g_{4,(0,1,0,0)} g_{2,(0,0,0,0)}, \\
\mathbf{e}_{5}=g_{3,(0,0,1,0)} g_{3,(0,0,0,0)}, & \mathbf{e}_{6}=g_{3,(0,0,0,1)} g_{3,(0,0,0,0)} .
\end{array}
$$

Thus for any sector, the conditions

$$
\Phi_{g_{f}}=1 \quad \text { for all fixed points elements } g_{f} \in S,
$$

are a reformulation of the projection conditions to the zero modes in four dimensions. For the above bulk fields in the effective field theory on $\mathcal{M}_{\text {eff }}$, this statement coincides with the familiar orbifold boundary conditions.

At intermediate scales between the compactification scales of the large and the small dimensions of the anisotropic orbifold, the full string compactification including all massive modes should be described by the effective orbifold field theory on $\mathcal{M}_{\text {eff }}$ to good approximation. In the following, we calculate the corresponding spectrum by solving

Localized states : $\quad \Phi_{g_{f^{\prime}}}=1 \quad$ for all fixed point elements $g_{f^{\prime}} \in S$,

Bulk states : $\quad \Phi_{g_{f^{\prime}}}=1 \quad$ for all $g_{f^{\prime}}$ : no localization in $T_{\text {large }}^{n}$,

Projection to $\mathbf{z}_{g_{f}}: \quad \Phi_{g_{f^{\prime}}}=1 \quad$ for all $g_{f^{\prime}}$ : localization at $\mathbf{z}_{g_{f}}$ in $T_{\text {large }}^{n}$,

Zero modes : $\quad \Phi_{g_{f}}=1 \quad$ for all $g_{f}:$ localization in $T_{\text {large }}^{n}$.

Here $f, f^{\prime}=(k, \mathbf{n})$ correspond to one of the 44 fixed point sectors of the model and $g_{f}, g_{f^{\prime}}$ are some representatives of the associated conjugacy classes. Note that localized states as well as bulk states can arise from several inequivalent sectors. Each of them has different phases $\Phi_{h}$ and is constrained by an independent set of conditions.

The last two conditions apply to bulk states and require that (134b) is fulfilled simultaneously. If the projection (134c) is evaluated for all fixed point elements in the plane, the solutions are zero modes of the orbifold.

Alternatively to the condition (134a) for states localized at $\mathbf{z}_{g_{f}}$ in $\mathcal{M}_{\text {eff }}$, which is the same as the projection to zero modes in four dimensions, one could discuss the condition

$$
\Phi_{g_{f^{\prime}}}=1 \quad \text { for all } g_{f^{\prime}}: \text { localization at } \mathbf{z}_{g_{f}} \text { in } T_{\text {large }}^{n} .
$$

The difference to (134a) is that there, compatibility with other fixed point elements with localization in $T_{\text {large }}^{n}$ is required. This assigns the corresponding translational phases $\gamma_{a}^{\prime}$ to the states. One observes that the assignment does commute with the four-dimensional gauge symmetry, but not with the larger local gauge symmetries at the orbifold fixed points. The reason for that is that precisely the lattice translations which imply the difference between the local gauge symmetries and the effective gauge symmetry in the lowenergy limit are also responsible for the choice of $\gamma^{\prime}$, for a state with given charge vectors. 


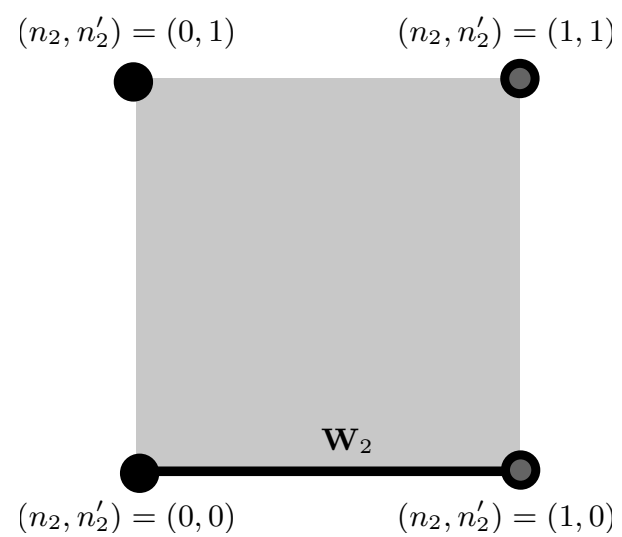

Figure 3: Sketch of the geometry of the effective co-dimension two $\mathbb{Z}_{2}$ orbifold. It corresponds to the surface of a pillow, which means that there is a back side to the gray bulk region, cf. Figure 2. The model has one non-vanishing Wilson line $\mathbf{W}_{2}$, which implies that the fixed points with the same label $n_{2}$ are equivalent, the other ones are not.

However, what we are aiming at is an effective description for the compactification of the string on a six-dimensional orbifold, so the assignment cannot be avoided for a consistent description. We conclude that the $\gamma_{a}^{\prime}$ values in the directions of $T_{\text {large }}^{n}$ are properties of the four-dimensional zero mode limit, rather than quantum numbers of the local theory at a fixed point.

\subsubsection{Geometry of the effective GUT}

Starting from the $\mathbb{Z}_{6-\text { II }}$ geometry, described in Section 3.1, we now propose an effective co-dimension two orbifold model. In the language of (129) it corresponds to the choice (we now switch back to complex coordinates, but still label tori by their real dimension)

$$
T_{\text {small }}^{4}=\mathbb{C}^{2} / \Gamma_{G_{2} \times \mathrm{SU}(3)}, \quad T_{\text {large }}^{2}=\mathbb{C} / \Gamma_{\mathrm{SO}(4)},
$$

where the subscripts 'large' and 'small' refer to a scale comparable to the GUT scale, and a much smaller scale, related to the string or the Planck scale, respectively. The exact choice should eventually be compatible with precision gauge coupling unification in the resulting model, and the perturbative string description.

The $\mathbb{Z}_{6-\text { II }}$ twist contains a $\mathbb{Z}_{2}$ and a $\mathbb{Z}_{3}$ sub-twist, cf. (103),

$$
\begin{aligned}
& \mathbb{Z}_{2}: \quad \mathbf{v}_{2}=\left(-\frac{1}{2},-1, \frac{3}{2} ; 0\right), \quad \text { transforms } T_{\text {small }}^{4} \text { and } T_{\text {large }}^{2}, \\
& \mathbb{Z}_{3}: \quad \mathbf{v}_{3}=\left(-\frac{1}{3},-\frac{2}{3}, 1 ; 0\right), \quad \text { leaves } T_{\text {large }}^{2} \text { invariant } .
\end{aligned}
$$

The entries $v_{N}^{i}, i=1,2,3$, correspond to rotations in the $G_{2}, \mathrm{SU}(3)$ and $\mathrm{SO}(4)$-plane, respectively. The $\mathrm{SO}(4)$-plane is invariant under the $\mathbb{Z}_{3}$ in $\mathbb{Z}_{6-\mathrm{II}}$, and we can thus understand 
the effective $6 \mathrm{D}$ orbifold GUT limit as

$$
\mathcal{M}_{6}=\frac{\mathbb{C}^{2} /\left(\Gamma_{\mathrm{G}_{2} \times \mathrm{SU}(3)} \times \mathbb{Z}_{3}\right) \times \mathbb{C} / \Gamma_{\mathrm{SO}(4)}}{\mathbb{Z}_{2}} \quad \stackrel{T_{\text {small }}^{4} \text { small }}{\longrightarrow} \quad \mathcal{M}_{\mathrm{eff}}=\mathbb{C} /\left(\Gamma_{\mathrm{SO}(4)} \times \mathbb{Z}_{2}\right)
$$

Everything in the effective orbifold model is fixed by the underlying $\mathbb{Z}_{6-\text { II }}$ model, specified by the twist (102) and the gauge embedding (119). Note that the states at the fixed points of $\mathcal{M}_{\text {eff }}$ are invariant under $\mathbb{Z}_{6}$, while bulk states arise from modding out the $\mathbb{Z}_{3}$ subgroup.

The effective $\mathbb{Z}_{2}$ orbifold is sketched in Figure 3, where also the non-vanishing Wilson line in the $\mathbf{e}_{5}$ direction is indicated. This has the effect of shifting the local embedding of the $\mathbb{Z}_{2}$ at the fixed points with quantum number $n_{2}=1$ against the one at $n_{2}=0$. Since there is no second Wilson line in the $\mathbf{e}_{6}$ direction, fixed points with the same label $n_{2}$ are equivalent, in the sense that their spectra are identical.

\subsubsection{Generic projection conditions}

Every sector $\mathcal{H}_{[g]}$ is characterized by a set of labels

$$
\text { states at } \mathbf{z}_{g}: \quad k, n_{6}, n_{3}, n_{2}, n_{2}^{\prime}, l_{3}, l_{5}, l_{6}, \gamma, \mathbf{p}_{\mathrm{sh}}, \mathbf{R} .
$$

In the following we shall use the convention that $\gamma=0$ either corresponds to the untwisted sector, or to localization at the origin of the $\mathrm{G}_{2}$-plane, cf. Figure 2 . We write

$$
\gamma=\frac{j}{N_{(g)}}, \quad j=0 \quad \Leftrightarrow \quad n_{6}=0
$$

and $j=1, \ldots, N_{(g)}$ otherwise, which means if the localization in the $\mathrm{G}_{2}$-plane is described by $n_{6}=2$ or $n_{6}=3$. Furthermore, states which are delocalized in the $\mathrm{SU}(3)$ - or the $\mathrm{SO}(4)$ plane have $l_{3} \equiv 0$ or $l_{5} \equiv 0, l_{6} \equiv 0$ in all formulae, respectively. This is a consequence of the trivial sub-lattices for the states in these planes, which do not give rise to superpositions.

A general $g$ is related to states which are characterized by

$$
\begin{aligned}
\mathbf{p}_{\mathrm{sh}} & =\mathbf{p}+\mathbf{V}_{g}=\mathbf{p}+k\left(\mathbf{V}_{6}+n_{2} \mathbf{W}_{(2)}+n_{3} \mathbf{W}_{(3)}\right) \\
\mathbf{R} & =\mathbf{q}_{\mathrm{sh}}+\tilde{\mathbf{N}}^{*}-\tilde{\mathbf{N}}=k \mathbf{v}_{6}+\tilde{\mathbf{N}}^{*}-\tilde{\mathbf{N}}, \\
\gamma_{a}^{\prime} m_{a} & =\frac{l_{3}}{3} k n_{3}+\frac{l_{5}}{2} k n_{2}+\frac{l_{6}}{2} k n_{2}^{\prime} .
\end{aligned}
$$

We now aim to evaluate the projection condition $\Phi_{h}=1$ for these states. This we evaluate for all $h \in S$ or from a relevant subset, for example $h \in S_{\text {small }}$, defined in (130). The elements $h$ also have a specific set of labels,

$$
h: \quad \tilde{k}, \tilde{n}_{6}, \tilde{n}_{3}, \tilde{n}_{2}, \tilde{n}_{2}^{\prime},
$$

associated with

$$
\begin{aligned}
\mathbf{V}_{g_{f^{\prime}}} & =\mathbf{V}_{g}+(\tilde{k}-k) \mathbf{V}_{6}+\left(\tilde{k} \tilde{n}_{2}-k n_{2}\right) \mathbf{W}_{(2)}+\left(\tilde{k} \tilde{n}_{3}-k n_{3}\right) \mathbf{W}_{(3)}, \\
\mathbf{v}_{g_{f^{\prime}}} & =\mathbf{v}_{g}+(\tilde{k}-k) \mathbf{v}_{6} \\
\gamma_{a}^{\prime} \tilde{m}_{a} & =\frac{l_{3}}{3} \tilde{k} \tilde{n}_{3}+\frac{l_{5}}{2} \tilde{k} \tilde{n}_{2}+\frac{l_{6}}{2} \tilde{k} \tilde{n}_{2}^{\prime} .
\end{aligned}
$$


In a first step, one can solve the condition for $h=g$. This is a non-trivial projection of all available states onto physical subsets of charge vectors,

First step : $\quad \mathbf{p}_{\mathrm{sh}} \cdot \mathbf{V}_{g}-\mathbf{R} \cdot \mathbf{v}_{g}=0 \bmod 1 \Rightarrow$ solution for $\mathbf{p}_{\mathrm{sh}}, \mathbf{R}$.

Next, one has to consider $h \neq g$. In that case there may be a non-trivial geometrical phase,

$$
\tilde{\Phi}_{h}=e^{2 \pi i \frac{l_{3}}{3}\left(n_{3}-\tilde{n}_{3}\right) k \tilde{k}} e^{2 \pi i \frac{l_{5}}{2}\left(n_{2}-\tilde{n}_{2}\right) k \tilde{k}} e^{2 \pi i \frac{l_{6}}{2}\left(n_{2}^{\prime}-\tilde{n}_{2}^{\prime}\right) k \tilde{k}} e^{2 \pi i \gamma \tilde{k}} .
$$

With (143) one finds from $\Phi_{h}=1$ for $h$, taken from a subset of $S$, that the additional geometric projection conditions are

$$
\begin{aligned}
\text { Second step : } & \begin{aligned}
\left(\tilde{n}_{2}^{\prime}-n_{2}^{\prime}\right) \frac{l_{6} k}{2} & =0 \bmod 1, \\
\left(\tilde{n}_{2}-n_{2}\right)\left(\mathbf{p}_{\mathrm{sh}} \cdot \mathbf{W}_{(2)}-\frac{l_{5} k}{2}\right) & =0 \bmod 1, \\
\left(\tilde{n}_{3}-n_{3}\right)\left(\mathbf{p}_{\mathrm{sh}} \cdot \mathbf{W}_{(3)}-\frac{l_{3} k}{3}\right) & =0 \bmod 1, \\
(\tilde{k}-k)\left(\mathbf{p}_{\mathrm{sh}} \cdot\left(\mathbf{V}_{6}+n_{2} \mathbf{W}_{(2)}+n_{3} \mathbf{W}_{(3)}\right)-\mathbf{R} \cdot \mathbf{v}_{6}+\frac{j}{N_{(g)}}\right) & =0 \bmod 1,
\end{aligned}
\end{aligned}
$$

for all $\tilde{k}, \tilde{n}_{3}, \tilde{n}_{2}, \tilde{n}_{2}^{\prime}$ that specify the considered elements $h$. Invariance under the full space group then corresponds to

$$
\begin{aligned}
\frac{l_{6} k}{2} & =0 \bmod 1 \\
\frac{l_{5} k}{2} & =\mathbf{p}_{\mathrm{sh}} \cdot \mathbf{W}_{(2)} \bmod 1 \\
\frac{l_{3} k}{3} & =\mathbf{p}_{\mathrm{sh}} \cdot \mathbf{W}_{(3)} \bmod 1 \\
\frac{j}{N_{(g)}} & =\left[\mathbf{R} \cdot \mathbf{v}_{6}-\mathbf{p}_{\mathrm{sh}} \cdot\left(\mathbf{V}_{6}+n_{2} \mathbf{W}_{(2)}+n_{3} \mathbf{W}_{(3)}\right)\right] \bmod 1
\end{aligned}
$$

Note that for bulk states in a plane the corresponding geometrical quantum numbers $l_{i}$ are zero. This may imply further projection conditions for the charge vectors $\mathbf{p}_{\mathrm{sh}}, \mathbf{R}$. Since localization in the $\mathrm{SO}(4)$-plane requires $k=1,3,5$, one always finds $l_{6}=0 \bmod 2$ in our model. We can thus completely ignore this quantum number in the following. This is a consequence of the vanishing Wilson line $\mathbf{W}_{(2)}^{\prime}$, associated with the lattice vector $\mathbf{e}_{6}$.

The geometrical projection conditions fix the phases $\gamma^{\prime}, \gamma$ for physical states. A sketch of this two-step projection procedure was given in (75). The result states that non-trivial phases in translational superpositions only appear if Wilson lines are present. On first sight, the introduction of $\gamma^{\prime}, \gamma$ enlarges the physical Hilbert space since the phases correspond to new eigenstates of the space group operators, which are overseen if one restricts to the case 
of unit coefficients. This larger multiplicity is then reduced by the consistency conditions in the presence of Wilson lines, and in the end the number of solutions is not increased due to the new phases. However, we stress that for orbifolds with non-vanishing Wilson lines all geometrical phases are present and take non-trivial values ${ }^{14}$.

\subsubsection{The gravity sector}

The gravity sector of the six dimensional effective theory consists of the supergravity multiplet, a dilaton multiplet (also called tensor multiplet) and gravitational moduli related to the four small compact dimensions. All these states are uncharged under gauge symmetries and arise from the untwisted sector. As discussed in section 2.3.4, they are of the form

$$
|\mathbf{p}=0, \mathbf{R}=\mathbf{q}+\underbrace{(1,0,0,0)}_{\tilde{\mathbf{N}}^{*}}\rangle, \quad|\mathbf{p}=0, \mathbf{R}=\mathbf{q}-\underbrace{(1,0,0,0)}_{\tilde{\mathbf{N}}}\rangle,
$$

with $\gamma^{\prime}=0, \gamma=0$, and oscillator numbers $\tilde{\mathbf{N}}^{*}, \tilde{\mathbf{N}}$. They correspond to excitations associated to operators $\alpha_{-1}^{* i}, \alpha_{-1}^{i}, i=1, \ldots, 4$, respectively. The right-moving momenta $\mathbf{q}$ are constrained by the conditions (79), (80), which state that the states have to be massless. Solutions for $\mathbf{q}$ take the form (82) and correspond to representations $\boldsymbol{8}_{v}+\boldsymbol{8}_{s}$ of $\mathrm{SO}(8)$, the oscillators form another $\mathbf{8}_{v}$.

These states are now subject to the projection condition (134b). It has to be evaluated for all fixed point elements $g_{f^{\prime}}$ for which the $\mathrm{SO}(4)$-plane is a fixed torus. These are the ones with $\tilde{k}=2,4$, since that property is related to the $\mathbb{Z}_{3}$ which leaves $T_{\text {large }}^{2}$ invariant. Untwisted states have $k=0, l_{3}=0, l_{5}=0, j=0, \tilde{n}_{2}=n_{2}=0$, and the conditions (146) reduce to

$$
\left(\mathbf{q}+\tilde{\mathbf{N}}^{*}-\tilde{\mathbf{N}}\right) \cdot \mathbf{v}_{3}=0 \bmod 1
$$

First, consider solutions with excitations $\alpha_{-1}^{i}, \alpha_{-1}^{* i}$ in the large planes, $i=3,4$. These operators will again transform in the vector representation of the little group of the Lorentz group for the six large dimensions. The latter is given by $\mathrm{SO}(4)=\mathrm{SU}(2) \times \mathrm{SU}(2)$,

$$
\mathrm{SO}(8) \rightarrow \mathbb{Z}_{3} \times \mathrm{SO}(4)=\mathbb{Z}_{3} \times \mathrm{SU}(2) \times \mathrm{SU}(2),
$$

and the right-moving momenta are

$$
\mathbf{q}=\underbrace{(0,0, \pm 1,0)}_{(\mathbf{2}, \mathbf{2}) \text { of } \mathrm{SU}(2) \times \mathrm{SU}(2)} \quad \text { or } \quad \mathbf{q}=\underbrace{ \pm\left(\frac{1}{2}, \frac{1}{2}, \frac{1}{2}, \frac{1}{2}\right), \pm\left(\frac{1}{2}, \frac{1}{2},-\frac{1}{2},-\frac{1}{2}\right)}_{2 \cdot(\mathbb{1}, \mathbf{2}) \text { of } \mathrm{SU}(2) \times \mathrm{SU}(2)},
$$

\footnotetext{
${ }^{14}$ If one is only interested in the results for the spectrum, one can decide to ignore the translational $\gamma^{\prime}$ phases for localized states from the start, and simultaneously also the projection conditions for elements $h \neq g$ in a sector $\mathcal{H}_{[g]}$. However, for bulk states one still requires the rules $\mathbf{p}_{\mathrm{sh}} \cdot \mathbf{W}_{a}=0 \bmod 1$. This is indeed a popular approach in the literature, cf. [13,14,49].
} 
where the fermionic states correspond to a left-handed Weyl fermion in six dimensions. Thus in total, one recovers the contents of the supergravity multiplet and the dilaton multiplet in six dimensions $[8,63]$ :

$$
\begin{aligned}
(\mathbf{2}, \mathbf{2}) \times(\mathbf{2}, \mathbf{2}) & =\underbrace{(\mathbf{3}, \mathbf{3})}_{g_{M N}}+\underbrace{(\mathbf{3}, \mathbb{1})}_{B_{M N}^{+}}+\underbrace{(\mathbb{1}, \mathbf{3})}_{B_{M N}^{-}}+\underbrace{(\mathbb{1}, \mathbb{1})}_{\phi}, \\
(\mathbf{2}, \mathbf{2}) \times[2 \cdot(\mathbb{1}, \mathbf{2})] & =\underbrace{2 \cdot(\mathbf{2}, \mathbf{3})}_{\tilde{g}_{M}}+\underbrace{2 \cdot(\mathbf{2}, \mathbb{1})}_{\tilde{\phi}} .
\end{aligned}
$$

Here $B_{M N}$ denotes the antisymmetric two-form, which can be split into an anti-self-dual part $B_{M N}^{-}$and a self-dual part $B_{M N}^{+}$. These contributions appear in the supergravity multiplet and the tensor multiplet, respectively, where the latter multiplet contains also the dilaton $\phi$. The fermions are given by the gravitino $\tilde{g}_{M}$ in six dimensions and the dilatino $\tilde{\phi}$,

Gravity multiplet: $\quad\left(g_{M N}, B_{M N}^{-}, \tilde{g}_{M}\right), \quad$ Tensor multiplet: $\left(B_{M N}^{+}, \phi, \tilde{\phi}\right)$.

Note that the number of bosonic degrees of freedom equals the number of fermionic ones within each of these multiplets.

Second, the projection condition (149) also has solutions with excitations in the internal directions,

$$
\left\{\begin{array}{ll}
\tilde{\mathbf{N}}=(1,0,0,0), & \tilde{\mathbf{N}}^{*}=0, \\
\tilde{\mathbf{N}}=0, & \tilde{\mathbf{N}}^{*}=(1,0,0,0)
\end{array}\right\} \quad \text { or } \quad\left\{\begin{array}{ll}
\tilde{\mathbf{N}}=(0,1,0,0), & \tilde{\mathbf{N}}^{*}=0, \\
\tilde{\mathbf{N}}=0, & \tilde{\mathbf{N}}^{*}=(0,1,0,0)
\end{array}\right\} .
$$

For each of these pairs of choices for $\tilde{\mathbf{N}}, \tilde{\mathbf{N}}^{*}$, the right-moving momenta of four bosonic degrees of freedom and a right-handed Weyl fermion in six dimensions are given by

$$
\mathbf{q}=\underbrace{( \pm 1,0,0,0)}_{4 \cdot(\mathbb{1}, \mathbb{1}) \text { of } \mathrm{SU}(2) \times \mathrm{SU}(2)} \quad \text { or } \quad \mathbf{q}=\underbrace{ \pm\left(\frac{1}{2},-\frac{1}{2}, \underline{\left.\frac{1}{2},-\frac{1}{2}\right)}\right.}_{2 \cdot(\mathbf{2}, \mathbb{1}) \text { of } \mathrm{SU}(2) \times \mathrm{SU}(2)} .
$$

These $\mathbf{q}$ represent the eight degrees of freedom that are contained in one hypermultiplet in six dimensions ${ }^{15}$. Note that the oscillator numbers of two states with opposite momenta correspond to excitations in complex conjugated directions, $(\mathbf{q}, \tilde{\mathbf{N}}) \leftrightarrow\left(-\mathbf{q}, \tilde{\mathbf{N}}^{*}\right)$. The above solutions give rise to two hypermultiplets,

$$
C_{1}, C_{2}
$$

which are related to excitations in the planes $G_{2}$ and $\mathrm{SU}(3)$, respectively. The associated eight bosonic degrees of freedom are the two corresponding volume moduli and off-diagonal fluctuations of the metric and the antisymmetric tensor. Note that there are no complex structure moduli, since the geometry of the model is fixed to the orbifold.

\footnotetext{
${ }^{15}$ This is twice the content of a half-multiplet $2 \cdot(\mathbb{1}, \mathbb{1})+(\mathbf{2}, 1)$, cf. appendix B.7 of [8].
} 


\subsubsection{Gauge vectors}

The gauge symmetry in the six-dimensional bulk is identified by analysis of the massless vector representations of the $\mathrm{SO}(4)$ Lorentz transformations, which survive the orbifold projection. These states come from the untwisted sector and hence have $\gamma^{\prime}=\gamma=0$. There are 16 Cartan generators which survive the projection, as it was the case for $\mathrm{E}_{8} \times \mathrm{E}_{8}$, but with right-moving momenta restricted by (149),

$$
\begin{aligned}
& 16 \text { Cartan generators: } \quad \alpha_{-1}^{I}|\mathbf{p}=0, \mathbf{R}=\mathbf{q}\rangle, \quad I=1, \ldots, 16, \\
& \mathbf{q}=\underbrace{(0,0, \pm 1,0)}_{(\mathbf{2}, \mathbf{2}) \text { of } \mathrm{SU}(2) \times \mathrm{SU}(2)} \quad \text { or } \quad \mathbf{q}=\underbrace{ \pm\left(\frac{1}{2}, \frac{1}{2}, \frac{1}{2}, \frac{1}{2}\right), \pm\left(\frac{1}{2}, \frac{1}{2},-\frac{1}{2},-\frac{1}{2}\right)}_{2 \cdot(\mathbb{1}, \mathbf{2}) \text { of } \mathrm{SU}(2) \times \mathrm{SU}(2)},
\end{aligned}
$$

These $\mathbf{q}$ vectors describe a vector multiplet in six dimensions [8].

Next, we find the states with the same right-moving momenta, but also non-vanishing left-moving momenta $\mathbf{p}$,

$$
|\mathbf{p}, \mathbf{q}\rangle: \quad \mathbf{q} \text { from }(157), \quad \mathbf{p} \cdot \mathbf{V}_{g_{f^{\prime}}}=0 \bmod 1 .
$$

The latter condition is the projection condition (134b), $\Phi_{g_{f^{\prime}}}=1$, evaluated for fixed point elements $g_{f^{\prime}}$ which leave the $\mathrm{SO}(4)$-plane invariant. Their local shift vectors were defined in $(120)$,

$$
\mathbf{V}_{g_{f^{\prime}}}=\tilde{k}\left(\mathbf{V}_{6}+\tilde{n}_{3} \mathbf{W}_{(3)}\right), \quad \tilde{k}=2,4, \quad \tilde{n}_{3}=0,1,2 .
$$

Thus (158) is a set of six conditions on the vectors $\mathbf{p}$. They are equivalent to two equations, $\mathbf{p} \cdot \mathbf{V}_{3}=0 \bmod 1, \mathbf{p} \cdot \mathbf{W}_{(3)}=0 \bmod 1$, where $\mathbf{V}_{3} \equiv 2 \mathbf{V}_{6}$. The same conditions follow from (146), with $k=0, l_{3}=0, l_{5}=0, j=0, n_{2}=\tilde{n}_{2}=0$. There are $30^{2}$ solutions,

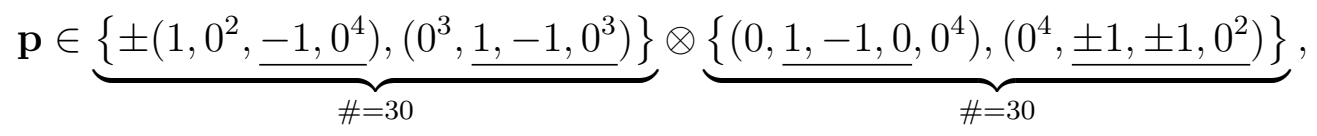

where we introduced the notation $0^{n}$ for $n$ subsequent entries of zero.

The solutions (160) are a subset of the roots of $E_{8} \times E_{8}$. In fact, they are the roots of a subgroup, whose embedding is a consequence of the specific form of the shift vector and the Wilson line. We now identify this subgroup by standard methods, as collected in Appendix B. For that we isolate the simple roots $\mathbf{p}_{i}$ among the above set of left-moving momenta,

$$
\begin{aligned}
& \mathbf{p}_{i} \in\{\underbrace{\left(1,0^{2},-1,0^{4}\right),\left(0^{3}, 1,-1,0^{3}\right),\left(0^{4}, 1,-1,0^{2}\right),\left(0^{5}, 1,-1,0\right),\left(0^{6}, 1,-1\right)}_{\mathrm{SU}(6)}\} \\
& \otimes\{\underbrace{\left(0,1,-1,0^{5}\right),\left(0^{2}, 1,-1,0^{4}\right)}_{\mathrm{SU}(3)}, \underbrace{\left(0^{4}, 1,-1,0^{2}\right),\left(0^{5}, 1,-1,0\right),\left(0^{6}, 1,-1\right),\left(0^{6}, 1,1\right)}_{\mathrm{SO}(8)}\},
\end{aligned}
$$




\begin{tabular}{c|l|c|c|c|c}
$\mathrm{U}(1)$ & Generator Embedding into $E_{8} \times E_{8}$ & Bulk & $n_{2}=0$ & $n_{2}=1$ & $4 \mathrm{~d}$ \\
\hline \hline$t_{1}$ & $(0,1,0,0,0,0,0,0)(0,0,0,0,0,0,0,0)$ & $\sqrt{ }$ & $\sqrt{ }$ & $\sqrt{ }$ & $\sqrt{ }$ \\
\hline$t_{2}$ & $(0,0,1,0,0,0,0,0)(0,0,0,0,0,0,0,0)$ & $\sqrt{ }$ & $\sqrt{ }$ & $\sqrt{ }$ & $\sqrt{ }$ \\
\hline$t_{3}$ & $(1,0,0,1,1,1,1,1)(0,0,0,0,0,0,0,0)$ & $\sqrt{ }$ & $\sqrt{ }$ & $\sqrt{ }$ & $\sqrt{ }$ \\
\hline$t_{4}$ & $(0,0,0,0,0,0,0,0)(1,0,0,0,0,0,0,0)$ & $\sqrt{ }$ & $\sqrt{ }$ & $\sqrt{ }$ & $\sqrt{ }$ \\
\hline$t_{5}$ & $(0,0,0,0,0,0,0,0)(0,1,1,1,0,0,0,0)$ & $\sqrt{ }$ & $\sqrt{ }$ & $\sqrt{ }$ & $\sqrt{ }$ \\
\hline$t_{6}^{0}$ & $(5,0,0,-1,-1,-1,-1,-1)(0,0,0,0,0,0,0,0)$ & $\times$ & $\sqrt{ }$ & $\times$ & $\sqrt{ }$ \\
\hline$t_{6}^{1}$ & $(5,0,0,-10,-10,5,5,5)(0,0,0,0,0,0,0,0)$ & $\times$ & $\times$ & $\sqrt{ }$ & $\sqrt{ }$ \\
\hline$t_{7}$ & $(0,0,0,0,0,0,0,0)(0,1,1,-2,0,0,0,0)$ & $\times$ & $\times$ & $\sqrt{ }$ & $\sqrt{ }$ \\
\hline$t_{8}$ & $(0,0,0,0,0,0,0,0)(0,0,0,0,-1,-1,-1,1)$ & $\times$ & $\times$ & $\sqrt{ }$ & $\sqrt{ }$ \\
\hline$t_{Y}$ & $\left(0,0,0, \frac{1}{2}, \frac{1}{2},-\frac{1}{3},-\frac{1}{3},-\frac{1}{3}\right)(0,0,0,0,0,0,0,0)$ & $\times$ & $\times$ & $\times$ & $\sqrt{ }$ \\
\hline$t_{\text {an }}^{0}$ & $(5,0,-4,-1,-1,-1,-1,-1)(5,-1,-1,-1,0,0,0,0)$ & $\times$ & $\sqrt{ }$ & $\times$ & $\sqrt{ }$ \\
\hline$t_{\text {an }}^{1}$ & $(1,3,-1,1,1,1,1,1)(-4,4,4,4,0,0,0,0)$ & $\times$ & $\times$ & $\sqrt{ }$ & $\sqrt{ }$ \\
\hline$t_{\text {an }}^{(4 \mathrm{~d})}$ & $\left(\frac{11}{6}, \frac{1}{2},-\frac{3}{2},-\frac{1}{6},-\frac{1}{6},-\frac{1}{6},-\frac{1}{6},-\frac{1}{6}\right)\left(1, \frac{1}{3}, \frac{1}{3}, \frac{1}{3}, 0,0,0,0\right)$ & $\times$ & $\times$ & $\times$ & $\sqrt{ }$ \\
\hline$t_{X}$ & $\left(0,1,1,-\frac{2}{5},-\frac{2}{5},-\frac{2}{5},-\frac{2}{5},-\frac{2}{5}\right)\left(\frac{1}{2}, \frac{1}{6}, \frac{1}{6}, \frac{1}{6}, 0,0,0,0\right)$ & $\times$ & $\sqrt{ }$ & $\times$ & $\sqrt{ }$ \\
\hline$t_{B-L}$ & $\left(0,1,1,0,0,-\frac{2}{3},-\frac{2}{3},-\frac{2}{3}\right)\left(\frac{1}{2}, \frac{1}{6}, \frac{1}{6}, \frac{1}{6}, 0,0,0,0\right)$ & $\times$ & $\times$ & $\times$ & $\sqrt{ }$
\end{tabular}

Table 4: Definition of the U(1) generators. The last four columns indicate whether the generator is orthogonal to all generators of the semi-simple group $(\sqrt{ })$ in the bulk, at the fixed points or in four dimensions, or not $(\times)$. The anomalous $\mathrm{U}(1)$ 's are linear combinations of the commuting U(1)'s at the fixed point specified by the superscript or in four dimensions; they are denoted by $t_{\mathrm{an}}^{0}, t_{\mathrm{an}}^{1}$ and $t_{\mathrm{an}}^{(4 d)}$, respectively.

and read off the group from the corresponding Cartan matrix $A_{i j}=2 \mathbf{p}_{i} \cdot \mathbf{p}_{j} /\left|\mathbf{p}_{i}\right|^{2}$ :

$$
A_{i j}=\underbrace{\left(\begin{array}{rrrrr}
2 & -1 & 0 & 0 & 0 \\
-1 & 2 & -1 & 0 & 0 \\
0 & -1 & 2 & -1 & 0 \\
0 & 0 & -1 & 2 & -1 \\
0 & 0 & 0 & -1 & 2
\end{array}\right)}_{\mathrm{SU}(6)} \oplus \underbrace{\left(\begin{array}{rrrrrr}
2 & -1 & & & & \\
-1 & 2 & & & & \\
& & -1 & 0 & 0 \\
& & 2 & -1 & -1 \\
& & -1 & 2 & 0 \\
0 & -1 & 0 & 2
\end{array}\right)}_{\mathrm{SU}(3) \times \mathrm{SO}(8)} .
$$

Here the blank off-diagonal parts have zero entries. The Cartan matrices can be understood as definitions of the corresponding Lie groups. We conclude that the six-dimensional bulk theory has the gauge group

$$
G_{\text {bulk }}=\mathrm{SU}(6) \times \mathrm{U}(1)^{3} \times\left[\mathrm{SU}(3) \times \mathrm{SO}(8) \times \mathrm{U}(1)^{2}\right] .
$$

The U(1) factors have to be present since the total number of uncharged Cartan generators is unchanged by the orbifold projection condition, cf. (156). For example, SU(6) has rank 
five, and five Cartan generators together with the 30 states from (160) form the $\mathbf{3 5}$ of $\mathrm{SU}(6)$, the adjoint representation. The remaining three Cartan generators can only generate three additional U(1)'s in the visible sector (which means with origin in the first $E_{8}$ ). Similarly, two extra U(1)'s arise from the hidden sector (which means with origin in the second $\mathrm{E}_{8}$ ). The generators $t_{i}, i=1, \ldots, 5$, of the five $\mathrm{U}(1)$ factors have to be orthogonal to the simple roots (161). We choose a basis as specified in Table 4, which also shows a number of other Abelian factors that will become important later. The charge of a state $\left|\mathbf{p}_{\mathrm{sh}}\right\rangle$ under one of these $\mathrm{U}(1)$ 's with generator $t_{i}$ is then calculated by

$$
Q_{i}\left|\mathbf{p}_{\mathrm{sh}}\right\rangle=t_{i} \cdot H\left|\mathbf{p}_{\mathrm{sh}}\right\rangle=t_{i} \cdot \mathbf{p}_{\mathrm{sh}}\left|\mathbf{p}_{\mathrm{sh}}\right\rangle
$$

where $H$ denotes the vector of the 16 Cartan generators of $\mathrm{E}_{8} \times \mathrm{E}_{8}$.

Recalling that the $\mathbf{q}$ vectors in (158) imply a transformation as a vector multiplet in six dimensions, we have found the ingredients of a Super-Yang-Mills theory in the bulk, with gauge group (163). Note that the group factor $\mathrm{SU}(6)$ is large enough to inherit all standard model gauge fields.

\section{Gauge vector projections}

All gauge symmetry of the orbifold model has its origin in the vector multiplet of $\mathrm{E}_{8} \times \mathrm{E}_{8}$, which arises from the untwisted sector. However, at the orbifold fixed point the symmetry is generically reduced and some of the bulk states are projected out. This is required since at fixed points, supersymmetry is reduced and its breaking is related to the orbifold twist, in complete analogy to the local breaking of the gauge group.

For the fixed points $z_{g_{f}}^{i}$ of the effective GUT in $T_{\text {large }}^{2}$, this follows from the additional local projection conditions $(134 \mathrm{c})$. Let the elements $g_{f^{\prime}}$ which lead to a localization at $z_{g_{f}}^{i}$ be characterized by labels $\tilde{n}_{2}=n_{2}, \tilde{n}_{2}^{\prime}=n_{2}^{\prime}$, and

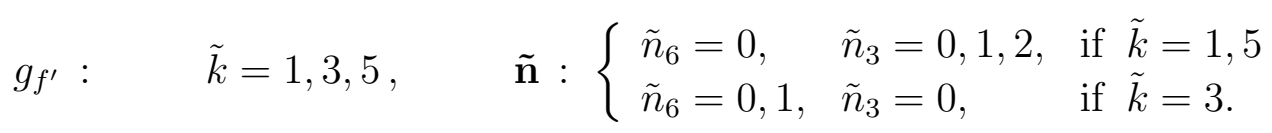

Then the additional conditions (146) become

$$
\tilde{k}\left(\mathbf{p} \cdot\left(\mathbf{V}_{6}+n_{2} \mathbf{W}_{(2)}\right)-\mathbf{q} \cdot \mathbf{v}_{6}\right)=0 \bmod 1, \quad \mathbf{p} \cdot \mathbf{W}_{(3)}=0 \bmod 1 .
$$

Here we are only interested in states which after the projection form vector multiplets at the fixed points, the remaining states will be discussed later. Local gauge vector states fulfill the additional constraint $\mathbf{q} \cdot \mathbf{v}_{6}=0 \bmod 1$, with solutions

$$
\mathbf{q}=(0,0,0, \pm 1), \quad \text { or } \quad \mathbf{q}= \pm\left(\frac{1}{2}, \frac{1}{2}, \frac{1}{2}, \frac{1}{2}\right)
$$

The left-moving momenta are then constrained by

$$
\mathbf{p} \cdot \mathbf{V}_{g_{f}}=0 \bmod 1, \quad \mathbf{p} \cdot \mathbf{W}_{(3)}=0 \bmod 1,
$$




\begin{tabular}{|c|c|c|}
\hline$n_{2}$ & non-Abelian gauge groups & Simple roots \\
\hline 0 & $S U(5) \times[S U(3) \times S O(8)]$ & $\begin{array}{c}\left\{\left(0^{3}, 1,-1,0^{3}\right),\left(0^{4}, 1,-1,0^{2}\right),\left(0^{5}, 1,-1,0\right),\left(0^{6}, 1,-1\right)\right\} \\
\otimes\left\{\left(0,1,-1,0^{5}\right),\left(0^{2}, 1,-1,0^{4}\right) ;\right. \\
\left.\left(0^{4}, 1,-1,0^{2}\right),\left(0^{5}, 1,-1,0\right),\left(0^{6}, 1,-1\right),\left(0^{6}, 1,1\right)\right\}\end{array}$ \\
\hline 1 & $S U(2) \times S U(4) \times\left[S U(2)^{\prime} \times S U(4)^{\prime}\right]$ & $\begin{array}{l}\left\{\left(0^{3}, 1,-1,0^{3}\right) ;\left(1,0^{4},-1,0^{2}\right),\left(0^{5}, 1,-1,0\right),\left(0^{6}, 1,-1\right)\right\} \\
\otimes\left\{\left(0,1,-1,0^{5}\right) ;\left(0^{4}, 1,-1,0^{2}\right),\left(0^{5}, 1,-1,0\right),\left(0^{6}, 1,1\right)\right\}\end{array}$ \\
\hline$\cap$ & $S U(2) \times S U(3) \times\left[S U(2)^{\prime} \times S U(4)^{\prime}\right]$ & $\begin{array}{c}\left\{\left(0^{3}, 1,-1,0^{3}\right) ;\left(0^{5}, 1,-1,0\right),\left(0^{6}, 1,-1\right)\right\} \\
\otimes\left\{\left(0,1,-1,0^{5}\right) ;\left(0^{4}, 1,-1,0^{2}\right),\left(0^{5}, 1,-1,0\right),\left(0^{6}, 1,1\right)\right\}\end{array}$ \\
\hline
\end{tabular}

Table 5: The local gauge groups at the fixed points $n_{2}=0$ and $n_{2}=1$, and their intersection. The latter is given by the product of the electroweak $\mathrm{SU}(2)$ and the color $\mathrm{SU}(3)$ of the standard model in the visible sector. The knowledge of the explicit embedding of the associated simple roots allows the calculation of the representations of matter states under the given gauge groups.

which has 50 solutions for $n_{2}=0$ and 28 for $n_{2}=1$. They again can be interpreted as simple roots of non-Abelian gauge groups, given in Table 5. The corresponding gauge groups are

$$
\begin{array}{rr}
n_{2}=0: & \mathrm{SU}(5) \times \mathrm{U}(1)^{4} \times\left[\mathrm{SU}(3) \times \mathrm{SO}(8) \times \mathrm{U}(1)^{2}\right], \\
n_{2}=1: & \mathrm{SU}(2) \times \mathrm{SU}(4) \times \mathrm{U}(1)^{4} \times\left[\mathrm{SU}(2)^{\prime} \times \mathrm{SU}(4)^{\prime} \times \mathrm{U}(1)^{4}\right],
\end{array}
$$

and the intersection in the visible sector contains the standard model gauge group,

$$
\mathrm{SU}(3) \times \mathrm{SU}(2) \times \mathrm{U}(1)^{5} \times\left[\mathrm{SU}(2)^{\prime} \times \mathrm{SU}(4)^{\prime} \times \mathrm{U}(1)^{4}\right] .
$$

The Abelian factors have generators $t_{i}$ which are defined in Table 4 . Note that the inequivalent fixed points $n_{2}=0$ and $n_{2}=1$ share the five $\mathrm{U}(1)$ factors from the bulk, but extend it by one more factor, with generator denoted by $t_{6}^{0}$ or $t_{6}^{1}$, respectively. The latter are neither collinear nor orthogonal in $\mathrm{E}_{8} \times \mathrm{E}_{8}$ weight space.

The spontaneous breakdown of all Abelian groups, except for hypercharge, under the constraint of a quasi-realistic phenomenology is the topic of the next chapter. Here we are interested in the spectrum of the model and the transformation of physical states under the full gauge groups given above.

In particular, we shall be interested in the SU(5) GUT which arises at the fixed points with $n_{2}=0$. Even though the underlying orbifold model [13] was designed to contain two generations of the standard model as 16-plets of $\mathrm{SO}(10)$, as will be apparent later, the anisotropic limit under consideration results in a local SU(5) model. The breaking to the standard model gauge group is due to the non-trivial Wilson line $\mathbf{W}_{(2)}$, or in other words, due to the existence of a second pair of inequivalent fixed points, corresponding to $n_{2}=1$. Since that is the only purpose of this second pair, and since it turns out that all matter which is localized at these fixed points is exotic and has to be decoupled, we do not expect that models with different Wilson lines $\mathbf{W}_{(2)}$ are fundamentally different in this class of compactifications. 


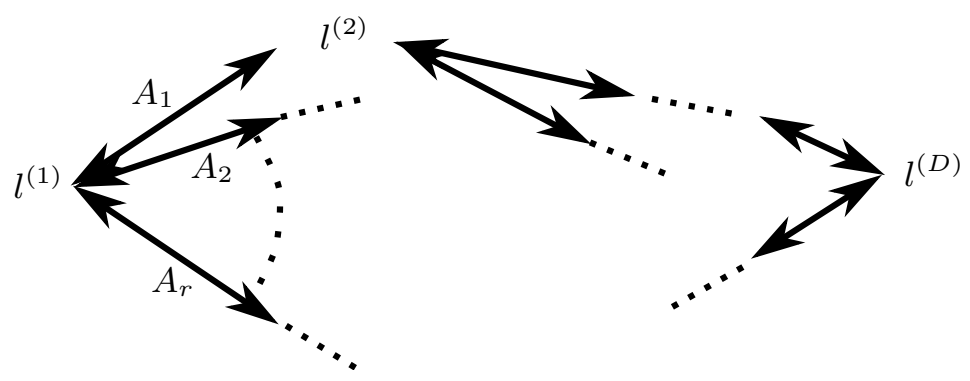

Figure 4: Sketch of a D-dimensional representation of a Lie group $G$. Shown are some of the Cartan-Weyl labels $l^{(n)}, n=1, \ldots, D$, defined in (172). They transform under the raising and lowering operators, represented by columns $A_{i}$ of the Cartan matrix, $i=1, \ldots, r=\operatorname{rank}(G)$. They arise from the subgroups $\mathrm{SU}(2)_{i}$, which are not orthogonal. Note that $A_{i}\left(-A_{i}\right)$ can only be applied if the $i$ - th entry of $l$ is negative (positive). Details can be found in Appendix $B$.

\subsubsection{Matter states}

Matter states $\left|\mathbf{p}_{\mathrm{sh}}, \mathbf{R}, \gamma^{\prime}, \gamma\right\rangle$ are in general charged under all or some of the generators associated with the relevant gauge group. We now describe how these states can be combined into multiplets.

As summarized in Appendix B, a representation is fully specified by its Dynkin labels $l=\left(l_{1}, \ldots, l_{r}\right)$, where $r$ is the total rank of the gauge group $G$. The vector $l$ arises from multiplication of the weight $\mathbf{p}_{\mathrm{sh}}$ with the simple roots $\mathbf{p}_{i}$, taken from (161) in the case of bulk states or from Table 5 for localized states. We evaluate the Cartan-Weyl labels $l_{i}$ for arbitrary weights, defined by

$$
l_{i} \equiv 2 \frac{\mathbf{p}_{i} \cdot \mathbf{p}_{\mathrm{sh}}}{\left|\mathbf{p}_{\mathbf{i}}\right|^{2}}, \quad \mathbf{p}_{i}: \text { simple roots of } G, \quad i=1, \ldots, r=\operatorname{rank}(G) .
$$

The Dynkin labels of a representation are then given by the vector $l$ of the highest weight state. Our approach here is to calculate $l$ for all present states, and then identify the ones which belong to the same representation of the relevant gauge group.

The $l_{i}$ are integer numbers. Recall that every simple root $\mathbf{p}_{i}$ is associated with a specific $\mathrm{SU}(2)_{i}$ subgroup of the gauge group, which can be associated with raising and lowering operators. The fact that all these $\mathrm{SU}(2)$ factors are interrelated is encoded in the offdiagonal entries of the Cartan matrix $A_{i j}$. The labels $l_{i}$ can then be understood as the number of times the lowering operator (or raising operator for $l_{i}<0$ ) which corresponds to $\mathrm{SU}(2)_{i}$ can be applied to the state, before it gives zero. The action of these operators on a state $l$ is given by subtracting (or adding) column number $i$ of the Cartan matrix, which we denote as $A_{i}$. In summary, a representation of dimension $D$ consists of $D$ states $^{16}$ with labels $l^{(n)}, n=1, \ldots, D$, which are related by the action of $A_{i}, i=1, \ldots, r$. A sketch of

\footnotetext{
${ }^{16}$ The adjoint representation is an exception, since the Cartan generators are not covered by the presented method. In that case, $D$ has to be replaced by the dimension of the group minus its rank.
} 
this relation is given in Figure 4. A simple example is the $\mathbf{3}$ of $\mathrm{SU}(3)$,

$$
l^{(1)}=\left(\begin{array}{l}
1 \\
0
\end{array}\right) \stackrel{-A_{1}}{\longmapsto} l^{(2)}=\left(\begin{array}{r}
-1 \\
1
\end{array}\right) \stackrel{-A_{2}}{\longmapsto} l^{(3)}=\left(\begin{array}{r}
0 \\
-1
\end{array}\right), \quad A_{i j}=\left(\begin{array}{rr}
2 & -1 \\
-1 & 2
\end{array}\right) .
$$

Similarly, we now calculate the representations that make up the sets $\left\{\mathbf{p}_{\text {sh }}\right\}$, which we obtain from solving the projection conditions for bulk matter and localized states.

\section{Bulk matter from the untwisted sector}

Not all states from the untwisted sector are contained in vector multiplets. The projection conditions $\Phi_{g_{f^{\prime}}}=1$ for states with $\mathbf{q} \cdot \mathbf{v}_{3} \neq 0 \bmod 1$ then give rise to hypermultiplets in six dimensions. With elements $g_{f^{\prime}}$ determined by the quantum numbers $\tilde{k}=2,4, \tilde{n}_{6}=0,2$, $\tilde{n}_{3}=0,1,2$ and $\tilde{n}_{2}=\tilde{n}_{2}^{\prime}=0$ the conditions (146) become

$$
\mathbf{p} \cdot \mathbf{V}_{3}-\mathbf{q} \cdot \mathbf{v}_{3}=0 \bmod 1, \quad \mathbf{p} \cdot \mathbf{W}_{(3)}=0 \bmod 1,
$$

where $l_{3}=j=n_{2}=0$ and $\tilde{\mathbf{N}}^{*}=\tilde{\mathbf{N}}=0$ for untwisted states was used. The conditions (174) have 384 solutions (p, q), which form 48 hypermultiplets with right-moving momenta $\mathbf{q}$ as in (154),

$$
\mathbf{q}=(\underline{ \pm 1,0}, 0,0) \quad \text { or } \quad \mathbf{q}= \pm\left(\frac{1}{2},-\frac{1}{2}, \frac{1}{2},-\frac{1}{2}\right)
$$

As described above, one can now calculate the labels $l_{i}$ by evaluating Equation (172) for each of the appearing left-moving momenta $\mathbf{p}_{\mathrm{sh}}=\mathbf{p}$. For example, consider $\mathbf{q}=$ $\left(-\frac{1}{2}, \frac{1}{2}, \frac{1}{2},-\frac{1}{2}\right)$ and

$$
\left.\begin{array}{l}
\mathbf{p}_{i}, \text { : simple roots of } \mathrm{SU}(6) \text { from }(161), \\
\mathbf{p}_{\mathrm{sh}}=\left(\frac{1}{2},-\frac{1}{2}, \frac{1}{2}, \frac{1}{2}, \frac{1}{2},-\frac{1}{2},-\frac{1}{2},-\frac{1}{2}\right)\left(0^{8}\right)
\end{array}\right\} \quad l=(0,0,1,0,0) .
$$

The resulting $l$ is the Dynkin label of the $\mathbf{2 0}$ of $\mathrm{SU}(6)$. In fact, there are exactly 19 other non-trivial $l$ vectors with respect to the simple roots of $\mathrm{SU}(6)$ among the 48 solutions, which transform as expected and complete the representation. We can now also calculate the five $\mathrm{U}(1)$ charges of the multiplet. According to (164) this is done by multiplying the vector $\mathbf{p}_{\mathrm{sh}}$ from (176) with the generators $t_{i}, i=1, \ldots, 5$, from Table 4 , with result

$$
\mathbf{Q} \equiv\left(t_{1} \cdot \mathbf{p}_{\mathrm{sh}}, \ldots, t_{5} \cdot \mathbf{p}_{\mathrm{sh}}\right)=\left(-\frac{1}{2}, \frac{1}{2}, 0,0,0\right)
$$

We have thus found a hypermultiplet $(\mathbf{2 0} ; \mathbb{1}, \mathbb{1})$ of $\mathrm{SU}(6) \times[\mathrm{SU}(3) \times \mathrm{SO}(8)]$, with charge vector $\mathbf{Q}$. The charge conjugated states have momenta $(-\mathbf{p},-\mathbf{q})$, and therefore inverted $\mathrm{U}(1)$ charges, $-\mathbf{Q}$.

The 28 remaining $\mathbf{p}$ vectors can be treated similarly. In summary, the untwisted matter states $|\mathbf{p}, \mathbf{q}\rangle$ are given by combinations of

$$
\begin{aligned}
& \mathbf{q}: 8 \text { states of a hypermultiplet, given in }(175), \\
& \mathbf{p}:(\mathbf{2 0} ; \mathbb{1}, \mathbb{1})+(\mathbb{1}, \mathbb{1}, \mathbf{8})+\left(\mathbb{1}, \mathbb{1}, \mathbf{8}_{s}\right)+\left(\mathbb{1}, \mathbb{1}, \mathbf{8}_{c}\right)+4 \cdot(\mathbb{1}, \mathbb{1}, \mathbb{1}) .
\end{aligned}
$$




\begin{tabular}{c|c||c|c|c|c|c}
\multicolumn{2}{c|}{ Multiplet } & $t_{1}$ & $t_{2}$ & $t_{3}$ & $t_{4}$ & $t_{5}$ \\
\hline \hline$(\mathbf{2 0}, \mathbb{1}, \mathbb{1})$ & & $-\frac{1}{2}$ & $\frac{1}{2}$ & 0 & 0 & 0 \\
$(\mathbb{1}, \mathbb{1}, \boldsymbol{8})$ & & 0 & 0 & 0 & -1 & 0 \\
$\left(\mathbb{1}, \mathbb{1}, \boldsymbol{8}_{s}\right)$ & & 0 & 0 & 0 & $\frac{1}{2}$ & $\frac{3}{2}$ \\
$\left(\mathbb{1}, \mathbb{1}, \boldsymbol{8}_{c}\right)$ & & 0 & 0 & 0 & $\frac{1}{2}$ & $-\frac{3}{2}$ \\
$(\mathbb{1}, \mathbb{1}, \mathbb{1})$ & $U_{1}$ & $\frac{1}{2}$ & $\frac{1}{2}$ & 3 & 0 & 0 \\
$(\mathbb{1}, \mathbb{1}, \mathbb{1})$ & $U_{2}$ & $\frac{1}{2}$ & $\frac{1}{2}$ & -3 & 0 & 0 \\
$(\mathbb{1}, \mathbb{1}, \mathbb{1})$ & $U_{3}$ & 1 & -1 & 0 & 0 & 0 \\
$(\mathbb{1}, \mathbb{1}, \mathbb{1})$ & $U_{4}$ & -1 & -1 & 0 & 0 & 0
\end{tabular}

Table 6: Bulk matter multiplets from the untwisted sector. Shown are states with $\mathbf{q}=$ $(-1,0,0,0),\left(-\frac{1}{2}, \frac{1}{2}, \frac{1}{2},-\frac{1}{2}\right) ;(0,1,0,0)\left(-\frac{1}{2}, \frac{1}{2},-\frac{1}{2}, \frac{1}{2}\right)$, corresponding to $H=\left(H_{L}, H_{R}\right)$. Additionally the charge conjugated states are present, such that each of the gauge multiplets appearing in the table corresponds to a full hypermultiplet. Singlets from the untwisted sector are called $U_{i}$.

Here the vector representation $\mathbf{8}$ of $\mathrm{SO}(8)$ with Dynkin labels $(1,0,0,0)$ appears, as well as the two spinor representations $\mathbf{8}_{s}, \boldsymbol{8}_{c}$, with Dynkin labels $(0,0,0,1)$ and $(0,0,1,0)$, respectively. The corresponding $\mathrm{U}(1)$ charges of the states can be inferred from Table 6 .

\section{Bulk matter from the twisted sectors}

We now consider bulk states $\left|\mathbf{p}_{\mathrm{sh}}, \mathbf{R} ; \gamma^{\prime}, \gamma\right\rangle_{[g]}$ of $T_{\text {large }}^{2}$ which are localized in $T_{\mathrm{small}}^{4}$. They correspond to twisted sectors, which are generated by space group elements $g=\left(\theta^{k}, m_{a} \mathbf{e}_{a}\right)$ with $k=2,4$, cf. Table 2 . The localization of the states on the orbifold is specified by the labels

$$
g: \quad k=2,4, \quad n_{6}=0,2, \quad n_{3}=0,1,2 .
$$

Additionally, states at $\mathbf{z}_{g}$ have the geometrical quantum numbers $\gamma^{\prime}$ and $\gamma$, specified by integers $l_{3}=0,1,2$ and $j=0,1,2$, respectively.

The projection condition for bulk states $(134 \mathrm{~b})$ requires $\Phi_{g_{f^{\prime}}}=1$ for all fixed point elements $g_{f^{\prime}}$ which leave $T_{\text {large }}^{2}$ invariant. Here we follow the two-step projection procedure presented in section 3.2.3. We first solve the condition (144) for $g_{f^{\prime}}=g$,

$$
\mathbf{p}_{\mathrm{sh}} \cdot \mathbf{V}_{g}-\mathbf{R} \cdot \mathbf{v}_{g}=0 \bmod 1
$$

For all $g$ from (180), this in total gives 6480 massless solutions. They form 810 hypermultiplets, which transform under the non-Abelian gauge group $\mathrm{SU}(6) \times[\mathrm{SU}(3) \times \mathrm{SO}(8)]$ 
as

$$
\begin{aligned}
& n_{6}=0: \quad 3 \cdot 3 \cdot[(\mathbf{6}, \mathbb{1}, \mathbb{1})+(\overline{\mathbf{6}}, \mathbb{1}, \mathbb{1})+(\mathbb{1}, \mathbf{3}, \mathbb{1})+(\mathbb{1}, \overline{\mathbf{3}}, \mathbb{1}) \\
& \left.+\left(\mathbb{1}, \mathbb{1}, \mathbf{8}^{*}\right)+4 \times(\mathbb{1}, \mathbb{1}, \mathbb{1})\right], \\
& n_{6}=2: \quad 2 \cdot 3 \cdot 3 \cdot[(\mathbf{6}, \mathbb{1}, \mathbb{1})+(\overline{\mathbf{6}}, \mathbb{1}, \mathbb{1})+(\mathbb{1}, \mathbf{3}, \mathbb{1})+(\mathbb{1}, \overline{\mathbf{3}}, \mathbb{1}) \\
& \left.+\left(\mathbb{1}, \mathbb{1}, \mathbf{8}^{*}\right)+4 \times(\mathbb{1}, \mathbb{1}, \mathbb{1})\right],
\end{aligned}
$$

where $\mathbf{8}^{*}=\mathbf{8}_{v}, \mathbf{8}_{s}, \boldsymbol{8}_{c}$, for $n_{3}=0,1,2$, respectively. Here the overall multiplicities arise from $n_{3} \in\{0,1,2\}, l_{3} \in\{0,1,2\}$ and $j=0$ for $n_{6}=0$, or $j \in\{1,2\}$ otherwise.

However, according to (134b) consistent bulk states are additionally constrained by $\Phi_{g_{f^{\prime}}}=1$, for other fixed point elements $g_{f^{\prime}} \neq g$. They also arise from the twisted sectors $T_{2}$ and $T_{4}$, and have $\tilde{n}_{3}=0,1,2, \tilde{n}_{2}=n_{2}=0$. This leads to the additional projection condition (146),

$$
\frac{l_{3} k}{3}=\mathbf{p}_{\mathrm{sh}} \cdot \mathbf{W}_{(3)} \bmod 1
$$

For a given vector $\mathbf{p}_{\mathrm{sh}}$ that is a solution of (181), this condition specifies the superposition in the $\mathrm{SU}(3)$ sub-lattice which is compatible with the orbifold symmetries, cf. (75). Thus the multiplicity of three in (182) and (183) which is related to $l_{3}$ is reduced to one. For each multiplet, one value of $l_{3} \in\{0,1,2\}$ is selected by (184).

Note that there is no similar restriction as (184) on the rotational superposition quantum number $j=0,1$ in this sector, neither do new constraints for the $R$-charges arise.

In total, we have found 270 hypermultiplets from the $T_{2}$ and $T_{4}$ twisted sectors, which are bulk states of the six-dimensional effective theory. Their quantum numbers and gauge charges are summarized in Table 7.

Matter states at $n_{2}=0$

We now consider matter states from a sector $\mathcal{H}_{\left[g_{f}\right]}$ which are localized at the fixed point $n_{2}=0$ in the large plane $T_{\text {large }}^{2}$. These are massless states (79), (80), which fulfill the projection conditions (134a),

$$
\begin{gathered}
\Phi_{g_{f^{\prime}}}=e^{2 \pi i\left(\mathbf{p}_{\mathrm{sh}} \cdot \mathbf{v}_{g_{f^{\prime}}}-\mathbf{R} \cdot \mathbf{v}_{g_{f^{\prime}}}\right)} \tilde{\Phi}_{g_{f^{\prime}}}=1, \quad \tilde{\Phi}_{g_{f^{\prime}}}=e^{2 \pi i \gamma_{a}^{\prime}\left(\tilde{k} m_{a}-k \tilde{m}_{a}\right)} e^{2 \pi i \gamma \tilde{k}} \\
\text { for all fixed point elements } g_{f^{\prime}}=\left(\theta^{\tilde{k}}, \tilde{m}_{a} \mathbf{e}_{a}\right) \in S
\end{gathered}
$$

These states are also zero modes in four dimensions. The representative space group element $g_{f}$, which characterizes the localized states, can be chosen as in Table 2 . It is defined by the labels $k, \mathbf{n}=\left(n_{6}, n_{3}, n_{2}, n_{2}^{\prime}\right)$, with $n_{2}=0$ and

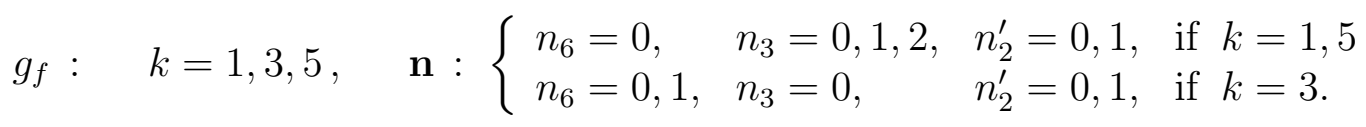




\begin{tabular}{|c|c|c|c|c|c|c|c|c|c|c|}
\hline Multiplet & $n_{3}$ & $n_{6} / \gamma$ & $\gamma_{3}^{\prime}$ & $t_{1}$ & $t_{2}$ & $t_{3}$ & $t_{4}$ & $t_{5}$ & $\tilde{\mathbf{N}}$ & $\tilde{\mathbf{N}}^{*}$ \\
\hline$(6, \mathbb{1}, \mathbb{1})$ & 0 & $0, \frac{1}{2}, 1$ & $\frac{1}{3}$ & 0 & $-\frac{1}{3}$ & 1 & $\frac{2}{3}$ & 0 & & \\
\hline$(\overline{\mathbf{6}}, \mathbb{1}, \mathbb{1})$ & 0 & $0, \frac{1}{2}, 1$ & 0 & 0 & $-\frac{1}{3}$ & -1 & $\frac{2}{3}$ & 0 & & \\
\hline$(\mathbb{1}, \mathbf{3}, \mathbb{1})$ & 0 & $0, \frac{1}{2}, 1$ & $\frac{1}{3}$ & 0 & $\frac{2}{3}$ & 0 & $-\frac{1}{3}$ & 1 & & \\
\hline$(\mathbb{1}, \overline{\mathbf{3}}, \mathbb{1})$ & 0 & $0, \frac{1}{2}, 1$ & 0 & 0 & $\frac{2}{3}$ & 0 & $-\frac{1}{3}$ & -1 & & \\
\hline$(\mathbb{1}, \mathbb{1}, \mathbf{8})$ & 0 & $0, \frac{1}{2}, 1$ & $\frac{2}{3}$ & 0 & $\frac{2}{3}$ & 0 & $-\frac{1}{3}$ & 0 & & \\
\hline$(\mathbb{1}, \mathbb{1}, \mathbb{1})$ & 0 & $0, \frac{1}{2}, 1$ & $\frac{2}{3}$ & 1 & $-\frac{1}{3}$ & 0 & $\frac{2}{3}$ & 0 & & \\
\hline$(\mathbb{1}, \mathbb{1}, \mathbb{1})$ & 0 & $0, \frac{1}{2}, 1$ & $\frac{2}{3}$ & -1 & $-\frac{1}{3}$ & 0 & $\frac{2}{3}$ & 0 & & \\
\hline$(\mathbb{1}, \mathbb{1}, \mathbb{1})$ & 0 & $0, \frac{1}{2}, 1$ & $\frac{2}{3}$ & 0 & $\frac{2}{3}$ & 0 & $\frac{2}{3}$ & 0 & $(0,1,0,0)$ & $(0,0,0,0)$ \\
\hline$(\mathbb{1}, \mathbb{1}, \mathbb{1})$ & 0 & $0, \frac{1}{2}, 1$ & $\frac{2}{3}$ & 0 & $\frac{2}{3}$ & 0 & $\frac{2}{3}$ & 0 & $(0,0,0,0)$ & $(1,0,0,0)$ \\
\hline$(6, \mathbb{1}, \mathbb{1})$ & 1 & $0, \frac{1}{2}, 1$ & $\frac{1}{3}$ & 0 & $-\frac{1}{3}$ & -1 & $-\frac{1}{3}$ & -1 & & \\
\hline$(\overline{\mathbf{6}}, \mathbb{1}, \mathbb{1})$ & 1 & $0, \frac{1}{2}, 1$ & 0 & $\frac{1}{2}$ & $\frac{1}{6}$ & 0 & $-\frac{1}{3}$ & -1 & & \\
\hline$(\mathbb{1}, \mathbf{3}, \mathbb{1})$ & 1 & $0, \frac{1}{2}, 1$ & $\frac{1}{3}$ & $-\frac{1}{2}$ & $\frac{1}{6}$ & 1 & $\frac{2}{3}$ & 0 & & \\
\hline$(\mathbb{1}, \overline{\mathbf{3}}, \mathbb{1})$ & 1 & $0, \frac{1}{2}, 1$ & 0 & $-\frac{1}{2}$ & $\frac{1}{6}$ & 1 & $-\frac{1}{3}$ & 1 & & \\
\hline$\left(\mathbb{1}, \mathbb{1}, \boldsymbol{8}_{s}\right)$ & 1 & $0, \frac{1}{2}, 1$ & $\frac{2}{3}$ & $-\frac{1}{2}$ & $\frac{1}{6}$ & 1 & $\frac{1}{6}$ & $\frac{1}{2}$ & & \\
\hline$(\mathbb{1}, \mathbb{1}, \mathbb{1})$ & 1 & $0, \frac{1}{2}, 1$ & $\frac{2}{3}$ & $\frac{1}{2}$ & $-\frac{5}{6}$ & 1 & $-\frac{1}{3}$ & -1 & & \\
\hline$(\mathbb{1}, \mathbb{1}, \mathbb{1})$ & 1 & $0, \frac{1}{2}, 1$ & $\frac{2}{3}$ & 0 & $\frac{2}{3}$ & -2 & $-\frac{1}{3}$ & -1 & & \\
\hline$(\mathbb{1}, \mathbb{1}, \mathbb{1})$ & 1 & $0, \frac{1}{2}, 1$ & $\frac{2}{3}$ & $-\frac{1}{2}$ & $\frac{1}{6}$ & 1 & $-\frac{1}{3}$ & -1 & $(0,1,0,0)$ & $(0,0,0,0)$ \\
\hline$(\mathbb{1}, \mathbb{1}, \mathbb{1})$ & 1 & $0, \frac{1}{2}, 1$ & $\frac{2}{3}$ & $-\frac{1}{2}$ & $\frac{1}{6}$ & 1 & $-\frac{1}{3}$ & -1 & $(0,0,0,0)$ & $(1,0,0,0)$ \\
\hline$(6, \mathbb{1}, \mathbb{1})$ & 2 & $0, \frac{1}{2}, 1$ & $\frac{1}{3}$ & $\frac{1}{2}$ & $\frac{1}{6}$ & 0 & $-\frac{1}{3}$ & 1 & & \\
\hline$(\overline{\mathbf{6}}, \mathbb{1}, \mathbb{1})$ & 2 & $0, \frac{1}{2}, 1$ & 0 & 0 & $-\frac{1}{3}$ & 1 & $-\frac{1}{3}$ & 1 & & \\
\hline$(\mathbb{1}, \mathbf{3}, \mathbb{1})$ & 2 & $0, \frac{1}{2}, 1$ & $\frac{1}{3}$ & $-\frac{1}{2}$ & $\frac{1}{6}$ & -1 & $-\frac{1}{3}$ & -1 & & \\
\hline$(\mathbb{1}, \overline{\mathbf{3}}, \mathbb{1})$ & 2 & $0, \frac{1}{2}, 1$ & 0 & $-\frac{1}{2}$ & $\frac{1}{6}$ & -1 & $\frac{2}{3}$ & 0 & & \\
\hline$\left(\mathbb{1}, \mathbb{1}, \boldsymbol{8}_{c}\right)$ & 2 & $0, \frac{1}{2}, 1$ & $\frac{2}{3}$ & $-\frac{1}{2}$ & $\frac{1}{6}$ & -1 & $\frac{1}{6}$ & $-\frac{1}{2}$ & & \\
\hline$(\mathbb{1}, \mathbb{1}, \mathbb{1})$ & 2 & $0, \frac{1}{2}, 1$ & $\frac{2}{3}$ & $\frac{1}{2}$ & $-\frac{5}{6}$ & -1 & $-\frac{1}{3}$ & 1 & & \\
\hline$(\mathbb{1}, \mathbb{1}, \mathbb{1})$ & 2 & $0, \frac{1}{2}, 1$ & $\frac{2}{3}$ & 0 & $\frac{2}{3}$ & 2 & $-\frac{1}{3}$ & 1 & & \\
\hline$(\mathbb{1}, \mathbb{1}, \mathbb{1})$ & 2 & $0, \frac{1}{2}, 1$ & $\frac{2}{3}$ & $-\frac{1}{2}$ & $\frac{1}{6}$ & -1 & $-\frac{1}{3}$ & 1 & $(0,1,0,0)$ & $(0,0,0,0)$ \\
\hline$(\mathbb{1}, \mathbb{1}, \mathbb{1})$ & 2 & $0, \frac{1}{2}, 1$ & $\frac{2}{3}$ & $-\frac{1}{2}$ & $\frac{1}{6}$ & -1 & $-\frac{1}{3}$ & 1 & $(0,0,0,0)$ & $(1,0,0,0)$ \\
\hline
\end{tabular}

Table 7: Bulk hypermultiplets from twisted sectors. All shown states have $k=2$ and $\mathbf{q}_{\mathrm{sh}}=$ $\left(-\frac{1}{3},-\frac{2}{3}, 0,0\right),\left(\frac{1}{6},-\frac{1}{6}, \frac{1}{2},-\frac{1}{2}\right) ;\left(\frac{2}{3}, \frac{1}{3}, 0,0\right),\left(\frac{1}{6},-\frac{1}{6},-\frac{1}{2}, \frac{1}{2}\right)$, corresponding to $H=\left(H_{L}, H_{R}\right)$. The charge conjugated states arise from the $k=4$ sector, so each gauge multiplet corresponds to a complete hypermultiplet. The case of a zero entry in the $n_{6} / \gamma$ column represents the case $n_{6}=\gamma=0$. non-vanishing entries specify the value of $\gamma \neq 0$ and imply $n_{6}=2$. Note that there are 18 bulk multiplets with non-vanishing oscillator numbers in internal directions. 
The corresponding states $\left|\mathbf{p}_{\mathrm{sh}}, \mathbf{R} ; \gamma^{\prime}, \gamma\right\rangle_{\left[g_{f}\right]}$ involve superposition quantum numbers $\gamma^{\prime}, \gamma$ which are specified by integers $l_{3}, l_{5}, j$,

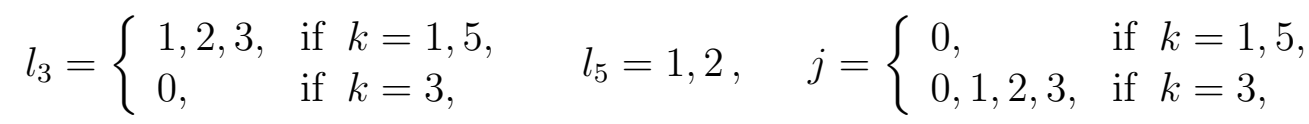

such that $\gamma_{a}^{\prime} m_{a}=\frac{l_{3}}{3} k n_{3}, \gamma=\frac{j}{3}$.

The above conditions can be solved by the two-step procedure of Section 3.2.3. First, one solves for $g_{f^{\prime}}=g$,

$$
\mathbf{p}_{\mathrm{sh}} \cdot \mathbf{V}_{g_{f}}-\mathbf{R} \cdot \mathbf{v}_{g_{f}}=0 \bmod 1
$$

and finds 3872 solutions, forming 968 chiral multiplets. These enormous numbers include multiplicities of three and four for $k=1,5$ and $k=3$, respectively, related to the constants $l_{3}, l_{5}, j$ from (187). We expect that this multiplicity is reduced by the additional constraints (147) due to $g_{f^{\prime}} \neq g_{f}$. These conditions are

$$
\begin{gathered}
\mathbf{p}_{\mathrm{sh}} \cdot\left(\mathbf{V}_{6}+n_{3} \mathbf{W}_{(3)}\right)-\mathbf{R} \cdot \mathbf{v}_{6}+\frac{j}{3}=0 \bmod 1, \\
\frac{l_{3} k}{3}=\mathbf{p}_{\mathrm{sh}} \cdot \mathbf{W}_{(3)} \bmod 1, \\
\frac{l_{5} k}{2}=\mathbf{p}_{\mathrm{sh}} \cdot \mathbf{W}_{(2)} \bmod 1,
\end{gathered}
$$

and indeed, for each solution they select specific values for the superposition quantum numbers $l_{3}, l_{5}, j$. However, for $k=3$ the value of $l_{3}$ is fixed to zero by (187), and a projection condition for $\mathbf{p}_{\mathrm{sh}}$ arises from the above:

$$
k=3: \quad \mathbf{p}_{\mathrm{sh}} \cdot \mathbf{W}_{(3)}=0 \bmod 1 .
$$

Note that for $k=1,5$ the value of $j=0$ is also fixed, but in that case (189) is identical to the projection condition (188), and not a new constraint.

With the full set of consistency conditions, 88 chiral multiplets survive at fixed points with $n_{2}=0$. Recall from Figure 3 that there are two equivalent fixed points with $n_{2}=0$ in the geometry. Since there is no Wilson line in the direction $\mathbf{e}_{6}$, their spectra are identical and thus consist of 44 chiral multiplets each. Up to the value for $l_{5}$, they form complete gauge representations with respect to the local gauge group ${ }^{17}$, as listed in Table 8. All listed states also appear in the four-dimensional zero mode spectrum. The latter was already studied in [13]. Appendix E repeats the findings in our notation, including the translational superposition quantum numbers $\gamma^{\prime}$.

The most striking feature of the local spectrum is the appearance of one complete standard model quark-lepton generation at each of the two fixed points with $n_{2}=0$. Recall from (169) that the local non-Abelian gauge group at these points is the unifying

\footnotetext{
${ }^{17} l_{5}$ is a quantum number of the four-dimensional theory and not of the local SU(5) GUT, since the simple root $\left(0^{4}, 1,-1,0^{2}\right)$ from Table 5 is not orthogonal to $\mathbf{W}_{(2)}$, cf. the discussion below (135).
} 


\begin{tabular}{c|c||c|c|c|c||c|c|c|c|c|c||c|c}
\multicolumn{2}{c|}{ Multiplet } & $k$ & $n_{3}$ & $\gamma$ & $\gamma_{3}^{\prime}$ & $t_{1}$ & $t_{2}$ & $t_{3}$ & $t_{4}$ & $t_{5}$ & $t_{6}^{0}$ & $\tilde{\mathbf{N}}$ & $\tilde{\mathbf{N}}^{*}$ \\
\hline \hline$(\mathbf{1 0}, \mathbb{1}, \mathbb{1})$ & $\mathbf{1 0}_{(1)}$ & 1 & 0 & 0 & 1 & 0 & $-\frac{1}{6}$ & $-\frac{1}{2}$ & $\frac{1}{3}$ & 0 & $\frac{1}{2}$ & & \\
$(\overline{\mathbf{5}}, \mathbb{1}, \mathbb{1})$ & $\overline{\mathbf{5}}_{(1)}$ & 1 & 0 & 0 & $\frac{2}{3}$ & 0 & $-\frac{1}{6}$ & $\frac{3}{2}$ & $\frac{1}{3}$ & 0 & $-\frac{3}{2}$ & & \\
$(\mathbb{1}, \mathbb{1}, \mathbb{1})$ & $N_{(1)}^{c}$ & 1 & 0 & 0 & $\frac{1}{3}$ & 0 & $-\frac{1}{6}$ & $-\frac{5}{2}$ & $\frac{1}{3}$ & 0 & $\frac{5}{2}$ & & \\
\hline$\left(\mathbb{1}, \mathbb{1}, \boldsymbol{8}_{c}\right)$ & & 1 & 1 & 0 & $\frac{1}{3}$ & 0 & $-\frac{1}{6}$ & $-\frac{1}{2}$ & $-\frac{1}{6}$ & $-\frac{1}{2}$ & $\frac{5}{2}$ & & \\
$(\mathbb{1}, \mathbf{3}, \mathbb{1})$ & & 1 & 2 & 0 & $\frac{2}{3}$ & 0 & $-\frac{1}{6}$ & $\frac{3}{2}$ & $\frac{1}{3}$ & 0 & $\frac{5}{2}$ & & \\
$(\mathbb{1}, \mathbb{1}, \mathbb{1})$ & $S_{8}$ & 1 & 2 & 0 & $\frac{2}{3}$ & 0 & $-\frac{1}{6}$ & $\frac{3}{2}$ & $-\frac{2}{3}$ & -1 & $\frac{5}{2}$ & & \\
\hline$(\mathbb{1}, \mathbb{1}, \mathbb{1})$ & $S_{1}$ & 1 & 0 & 0 & $\frac{1}{3}$ & $-\frac{1}{2}$ & $-\frac{2}{3}$ & $\frac{1}{2}$ & $\frac{1}{3}$ & 0 & $\frac{5}{2}$ & $(0,0,0,0)$ & $(1,0,0,0)$ \\
$(\mathbb{1}, \mathbb{1}, \mathbb{1})$ & $S_{2}$ & 1 & 0 & 0 & 1 & $\frac{1}{2}$ & $-\frac{2}{3}$ & $-\frac{1}{2}$ & $\frac{1}{3}$ & 0 & $-\frac{5}{2}$ & $(0,0,0,0)$ & $(1,0,0,0)$ \\
$(\mathbb{1}, \mathbb{1}, \mathbb{1})$ & $S_{3}$ & 1 & 0 & 0 & $\frac{1}{3}$ & $\frac{1}{2}$ & $\frac{1}{3}$ & $\frac{1}{2}$ & $\frac{1}{3}$ & 0 & $\frac{5}{2}$ & $(0,0,0,0)$ & $(0,1,0,0)$ \\
$(\mathbb{1}, \mathbb{1}, \mathbb{1})$ & $S_{4}$ & 1 & 0 & 0 & $\frac{1}{3}$ & $\frac{1}{2}$ & $\frac{1}{3}$ & $\frac{1}{2}$ & $\frac{1}{3}$ & 0 & $\frac{5}{2}$ & $(0,0,0,0)$ & $(2,0,0,0)$ \\
$(\mathbb{1}, \mathbb{1}, \mathbb{1})$ & $S_{5}$ & 1 & 0 & 0 & 1 & $-\frac{1}{2}$ & $\frac{1}{3}$ & $-\frac{1}{2}$ & $\frac{1}{3}$ & 0 & $-\frac{5}{2}$ & $(0,0,0,0)$ & $(0,1,0,0)$ \\
$(\mathbb{1}, \mathbb{1}, \mathbb{1})$ & $S_{6}$ & 1 & 0 & 0 & 1 & $-\frac{1}{2}$ & $\frac{1}{3}$ & $-\frac{1}{2}$ & $\frac{1}{3}$ & 0 & $-\frac{5}{2}$ & $(0,0,0,0)$ & $(2,0,0,0)$ \\
$(\mathbb{1}, \overline{\mathbf{3}}, \mathbb{1})$ & & 1 & 1 & 0 & 1 & 0 & $-\frac{1}{6}$ & $-\frac{1}{2}$ & $\frac{1}{3}$ & 0 & $\frac{5}{2}$ & $(0,0,0,0)$ & $(1,0,0,0)$ \\
$(\mathbb{1}, \mathbb{1}, \mathbb{1})$ & $S_{7}$ & 1 & 1 & 0 & $\frac{1}{3}$ & 0 & $-\frac{1}{6}$ & $-\frac{1}{2}$ & $-\frac{2}{3}$ & 1 & $\frac{5}{2}$ & $(0,0,0,0)$ & $(1,0,0,0)$ \\
\hline$(\mathbb{1}, \mathbf{3}, \mathbb{1})$ & & 3 & 0 & $\frac{1}{3}$ & 0 & $-\frac{1}{2}$ & 0 & $\frac{1}{2}$ & 0 & 1 & $\frac{5}{2}$ & & \\
$(\mathbb{1}, \overline{\mathbf{3}}, \mathbb{1})$ & & 3 & 0 & $\frac{1}{3}$ & 0 & $\frac{1}{2}$ & 0 & $-\frac{1}{2}$ & 0 & -1 & $-\frac{5}{2}$ & &
\end{tabular}

Table 8: Chiral multiplets at $\left(n_{2}, n_{2}^{\prime}\right)=(0,0)$ from the twisted sectors. A copy of this spectrum arises at $\left(n_{2}, n_{2}^{\prime}\right)=(0,1)$. Shown are states with $\mathbf{q}_{\mathrm{sh}}=\left(-\frac{1}{6},-\frac{1}{3},-\frac{1}{2}, 0\right),\left(\frac{1}{3}, \frac{1}{6}, 0,-\frac{1}{2}\right)$ for the case $k=1$, and $\mathbf{q}_{\mathrm{sh}}=\left(\frac{1}{2}, 0,-\frac{1}{2}, 0\right),\left(0, \frac{1}{2}, 0,-\frac{1}{2}\right)$ for $k=3$. The corresponding charge conjugate states have twist $6-k$ and are not listed. Two families of standard model chiral multiplets arise from $k=1, n_{3}=0$, with family index $(i)=(1),(2)$, for $n_{2}^{\prime}=0,1$, respectively. Furthermore, we introduce the nomenclature $S_{i}, S_{i}^{\prime}$ for localized singlets at $\left(n_{2}, n_{2}^{\prime}\right)=(0,0),(0,1)$, respectively. Note that seven out of eight gauge singlet chiral multiplets have oscillator numbers in internal directions. The value $\gamma=0$ implies $n_{6}=0$, states with $k=3$ have $n_{6}=1$.

group $\mathrm{SU}(5)$ in the visible sector. The only contribution with $k=1, n_{3}=0, \tilde{\mathbf{N}}=\mathbf{N}=0$ to the spectrum with respect to that group is

$$
\begin{array}{ll}
\left(n_{2}, n_{2}^{\prime}\right)=(0,0): & \mathbf{1 0}_{(1)}+\overline{\mathbf{5}}_{(1)}+N_{(1)}^{c}, \\
\left(n_{2}, n_{2}^{\prime}\right)=(0,1): & \mathbf{1 0}_{(2)}+\overline{\mathbf{5}}_{(2)}+N_{(2)}^{c},
\end{array}
$$

where $N_{(i)}^{c}$ denotes a right-handed neutrino and $(i)=(1),(2)$ is a family index. The third generation is combined from bulk states, as discussed in the next chapter.

\section{Matter states at $n_{2}=1$}

The fixed points with localization label $n_{2}=1$ play the role of 'hidden branes' in our model. This is meant in the sense that it is physically separated from the GUT fixed point, and all degrees of freedom which are localized there are exotics and have to be decoupled from the low-energy spectrum. The states at these fixed points are determined by the Wilson 
line $\mathbf{W}_{(2)}$, whose main purpose is to break the gauge group to the standard model gauge group in the low-energy limit. It has no direct effect on the spectrum at the SU(5) GUT fixed point. However, it does influence the zero modes of bulk fields and hence cannot be chosen arbitrarily for a valid phenomenology.

Here we take the viewpoint that there is much less to learn from the hidden brane than from the physical one. Once the details there are understood satisfactorily, we expect that a further adjustment of $\mathbf{W}_{(2)}$ can lead to further improvements.

In the following we shall thus restrict our attention mainly to the bulk and the GUT fixed points, characterized by $n_{2}=0$. However, for the sake of completeness the spectrum at the hidden brane for the chosen gauge embedding (119) is given in Appendix D. Here we just state the presence of exotics with non-vanishing charges under the electroweak $\mathrm{SU}(2)_{L}$ and the hypercharge $\mathrm{U}(1)_{Y}$ :

$$
\text { Exotics from } n_{2}=1: \quad 8 \cdot \mathbf{2}_{0}+16 \cdot\left(\mathbb{1}_{1 / 2}+\mathbb{1}_{-1 / 2}\right) .
$$

It is known that these states can be decoupled from the effective theory in four dimensions [13]. We assume that this is also possible in the situation discussed in this work, but do not study this issue any further.

\subsubsection{The effective orbifold GUT field theory}

In the previous sections, bulk and brane states on the geometry $\mathcal{M}_{\text {eff }}$ were calculated by solving the string mass equations and the relevant orbifold projection conditions. The assumption behind this construction was that the $\mathrm{SO}(4)$-plane is much larger than the $\mathrm{G}_{2^{-}}$ and $\mathrm{SU}(3)$-planes, whose scale is given by the string scale in our setup. Below that scale, one then has the effective description in terms of an orbifold field theory on $\mathbb{C} /\left(\Gamma_{\mathrm{SO}(4)} \times \mathbb{Z}_{2}\right)$. Note that the introduction of six-dimensional fields goes beyond the zero mode description and includes Kaluza-Klein modes of the two large compact dimensions.

All states which were found before are now interpreted as states which arise from corresponding fields on the six-dimensional space $T_{\text {large }}^{2} / \mathbb{Z}_{2} \times M_{4}$. For the $\mathcal{N}=2$ vector and hypermultiplets of the six-dimensional bulk we shall find it convenient to use a decomposition into $\mathcal{N}=1$ multiplets $[64]$

6d vector multiplet $A: A=(V, \phi), \quad\left\{\begin{array}{l}V: \text { 4d vector multiplet, } \\ \phi: 4 \mathrm{~d} \text { left-handed chiral multiplet, }\end{array}\right.$

6d hypermultiplet $H: \quad H=\left(H_{L}, H_{R}\right), \quad\left\{\begin{array}{l}H_{L}: \text { 4d left-handed chiral multiplet, } \\ H_{R}: \text { 4d right-handed chiral multiplet, }\end{array}\right.$

where $A$ transforms in the adjoint representation of the bulk gauge group (163), and $H$ as a multiplet from Tables 6,7 . The gauge interaction Lagrangian in the six-dimensional 


\begin{tabular}{c||c|c|c|c||c|c|c|c|c|c} 
Bulk & $n_{2}=0$ & $\mathrm{~V}$ & $\phi$ & $t_{6}{ }^{0}$ & $n_{2}=1$ & $\mathrm{~V}$ & $\phi$ & $t_{6}{ }^{1}$ & $t_{7}$ & $t_{8}$ \\
\hline \hline$(\mathbf{3 5}, \mathbb{1}, \mathbb{1})$ & $(\mathbf{2 4}, \mathbb{1}, \mathbb{1})$ & + & - & 0 & $(\mathbf{3}, \mathbb{1}, \mathbb{1}, \mathbb{1})$ & + & - & 0 & 0 & 0 \\
& $(\mathbf{5}, \mathbb{1}, \mathbb{1})$ & - & + & -6 & $(\mathbb{1}, \mathbf{1 5}, \mathbb{1}, \mathbb{1})$ & + & - & 0 & 0 & 0 \\
& $(\overline{\mathbf{5}}, \mathbb{1}, \mathbb{1})$ & - & + & 6 & $(\mathbf{2}, \mathbf{4}, \mathbb{1}, \mathbb{1})$ & - & + & 15 & 0 & 0 \\
& $(\mathbb{1}, \mathbb{1}, \mathbb{1})$ & + & - & 0 & $(\mathbf{2}, \overline{\mathbf{4}}, \mathbb{1}, \mathbb{1})$ & - & + & -15 & 0 & 0 \\
& & & & & $(\mathbb{1}, \mathbb{1}, \mathbb{1}, \mathbb{1})$ & + & - & 0 & 0 & 0 \\
\hline$(\mathbb{1}, \mathbf{8}, \mathbb{1})$ & $(\mathbb{1}, \mathbf{8}, \mathbb{1})$ & + & - & 0 & $(\mathbb{1}, \mathbb{1}, \mathbf{3}, \mathbb{1})$ & + & - & 0 & 0 & 0 \\
& & & & & $(\mathbb{1}, \mathbb{1}, \mathbf{2}, \mathbb{1})$ & - & + & 0 & 3 & 0 \\
& & & & & $(\mathbb{1}, \mathbb{1}, \mathbf{2}, \mathbb{1})$ & - & + & 0 & -3 & 0 \\
& & & & & $(\mathbb{1}, \mathbb{1}, \mathbb{1}, \mathbb{1})$ & + & - & 0 & 0 & 0 \\
\hline$(\mathbb{1}, \mathbb{1}, \mathbf{2 8})$ & $(\mathbb{1}, \mathbb{1}, \mathbf{2 8})$ & + & - & 0 & $(\mathbb{1}, \mathbb{1}, \mathbb{1}, \mathbf{1 5})$ & + & - & 0 & 0 & 0 \\
& & & & & $(\mathbb{1}, \mathbb{1}, \mathbb{1}, \mathbf{6})$ & - & + & 0 & 0 & 2 \\
& & & & & $(\mathbb{1}, \mathbb{1}, \mathbb{1}, \mathbf{6})$ & - & + & 0 & 0 & -2 \\
& & & & & $(\mathbb{1}, \mathbb{1}, \mathbb{1}, \mathbb{1})$ & + & - & 0 & 0 & 0
\end{tabular}

Table 9: Local decomposition of vector multiplets at $n_{2}=0$ and $n_{2}=1$. The representations refer to $\mathrm{SU}(6) \times[\mathrm{SU}(3) \times \mathrm{SO}(8)]$ in the bulk and $\mathrm{SU}(5) \times[\mathrm{SU}(3) \times \mathrm{SO}(8)]$ or $\mathrm{SU}(2) \times \mathrm{SU}(4) \times$ $\left[\mathrm{SU}(2)^{\prime} \times \mathrm{SU}(4)^{\prime}\right]$ at $n_{2}=0,1$, respectively. Shown are the charges under the strictly local $\mathrm{U}(1)$ factors and the parities under the local $\mathbb{Z}_{2}$ twist, for the $4 d \mathcal{N}=1$ components of the six-dimensional vector, $A=(V, \phi)$.

bulk can then be written as

$$
\begin{aligned}
\mathcal{L}_{H}= & \int d^{4} \theta\left(H_{L}^{\dagger} e^{2 g V} H_{L}+H_{R}^{c \dagger} e^{-2 g V} H_{R}^{c}\right) \\
& +\int d^{2} \theta H_{R}^{c}(\partial+\sqrt{2} g \phi) H_{L}+\text { h.c. },
\end{aligned}
$$

where $H_{L}^{c}, H_{R}^{c}$ are the charge conjugated fields.

The $\mathbb{Z}_{2}$ orbifold projection conditions at the fixed points $z_{g_{f}}^{3}$ in the $\mathrm{SO}(4)$-plane can now be formulated for the above bulk fields. For $g_{f}=\left(\theta^{k}, m_{a} \mathbf{e}_{a}\right), k=1,3,5$, and a coordinate $z^{3}$ in $T_{\text {large }}^{2}$ one has

$$
g_{f}\left(z_{g_{f}}^{3}+z^{3}\right)=z_{g_{f}}^{3}-z^{3},
$$

The local projection condition at $z_{g_{f}}^{3}$ for a generic bulk field $\phi\left(z^{3}\right)$ is then given by

$$
\begin{aligned}
P_{f}: \quad \phi\left(z_{g_{f}}^{3}+z^{3}\right) & =\eta_{f}(\phi) \phi\left(z_{g_{f}}^{3}-z^{3}\right), \\
\eta_{f}(\phi) & =e^{2 \pi i\left(\mathbf{p}_{\mathrm{sh}} \cdot \mathbf{V}_{6}-\mathbf{R} \cdot \mathbf{v}_{6}+\frac{j}{2}\right)},
\end{aligned}
$$

where $j=0$ for untwisted fields is understood. The above phase $\eta_{f}(\phi)$ has to be evaluated for the quantum numbers of the states associated with the field $\phi$. It follows from evaluating 


\begin{tabular}{c|c||c||c|c||c|c|c|c|c|c}
\multicolumn{2}{c|}{ Bulk } & $n_{2}=0$ & $H_{L}$ & $H_{R}$ & $t_{1}$ & $t_{2}$ & $t_{3}$ & $t_{4}$ & $t_{5}$ & $t_{6}^{0}$ \\
\hline \hline$(\mathbf{2 0}, \mathbb{1}, \mathbb{1})$ & & $(\mathbf{1 0}, \mathbb{1}, \mathbb{1})$ & + & - & $-\frac{1}{2}$ & $\frac{1}{2}$ & 0 & 0 & 0 & 3 \\
& & $(\overline{\mathbf{1 0}}, \mathbb{1}, \mathbb{1})$ & - & + & $-\frac{1}{2}$ & $\frac{1}{2}$ & 0 & 0 & 0 & -3 \\
\hline$(\mathbb{1}, \mathbb{1}, \mathbf{8})$ & & $(\mathbb{1}, \mathbb{1}, \mathbf{8})$ & - & + & 0 & 0 & 0 & -1 & 0 & 0 \\
\hline$\left(\mathbb{1}, \mathbb{1}, \boldsymbol{8}_{s}\right)$ & & $\left(\mathbb{1}, \mathbb{1}, \boldsymbol{8}_{s}\right)$ & + & - & 0 & 0 & 0 & $\frac{1}{2}$ & $\frac{3}{2}$ & 0 \\
\hline$\left(\mathbb{1}, \mathbb{1}, \boldsymbol{8}_{c}\right)$ & & $\left(\mathbb{1}, \mathbb{1}, \boldsymbol{8}_{c}\right)$ & + & - & 0 & 0 & 0 & $\frac{1}{2}$ & $-\frac{3}{2}$ & 0 \\
\hline$(\mathbb{1}, \mathbb{1}, \mathbb{1})$ & $U_{1}$ & $(\mathbb{1}, \mathbb{1}, \mathbb{1})$ & - & + & $\frac{1}{2}$ & $\frac{1}{2}$ & 3 & 0 & 0 & 0 \\
\hline$(\mathbb{1}, \mathbb{1}, \mathbb{1})$ & $U_{2}$ & $(\mathbb{1}, \mathbb{1}, \mathbb{1})$ & + & - & $\frac{1}{2}$ & $\frac{1}{2}$ & -3 & 0 & 0 & 0 \\
\hline$(\mathbb{1}, \mathbb{1}, \mathbb{1})$ & $U_{3}$ & $(\mathbb{1}, \mathbb{1}, \mathbb{1})$ & + & - & 1 & -1 & 0 & 0 & 0 & 0 \\
\hline$(\mathbb{1}, \mathbb{1}, \mathbb{1})$ & $U_{4}$ & $(\mathbb{1}, \mathbb{1}, \mathbb{1})$ & + & - & -1 & -1 & 0 & 0 & 0 & 0
\end{tabular}

Table 10: Chiral projection of bulk hypermultiplets from the untwisted sector to the fixed points $n_{2}=0$. The bulk and fixed point representations correspond to $\mathrm{SU}(6) \times[\mathrm{SU}(3) \times \mathrm{SO}(8)]$ and $\mathrm{SU}(5) \times[\mathrm{SU}(3) \times \mathrm{SO}(8)]$, respectively. Shown are the charges under the six local $\mathrm{U}(1)$ factors and the parities under the local $\mathbb{Z}_{2}$ twist, for the hypermultiplet components $H=$ $\left(H_{L}, H_{R}\right)$.

the condition (134c) for the bulk states with $k=0,2,4 ; n_{3}=0,1,2 ; \gamma_{3}^{\prime} k=\mathbf{p}_{\mathrm{sh}} \cdot \mathbf{W}_{(3)}$, and $j=0,1,2$, as found before. The corresponding projected matter states at the fixed point with $n_{2}=0$ are given in Tables 10, 11 and 12. A similar projection to the fixed points with $n_{2}=1$ is done in Appendix D.

The local parities of the vector fields with respect to the local $\mathbb{Z}_{2}$ twists are summarized in table 9 . This demonstrates how gauge symmetry breaking and supersymmetry breaking are linked. Consider for example the $\mathbf{3 5}$ of $\mathrm{SU}(6)$, the adjoint representation. In the six-dimensional bulk, the corresponding degrees of freedom can be expressed by a fourdimensional $\mathcal{N}=1$ vector multiplet $V$ and a chiral multiplet $\phi$, cf. (194). The parities in Table 9 now state that either the vector multiplet or the chiral multiplet survive the projection to one of the fixed points. Since at $n_{2}=0$ the gauge symmetry is broken to $\mathrm{SU}(5) \times \mathrm{U}(1)$, with adjoints $\mathbf{2 4}+\mathbb{1}$, this implies the presence of two additional chiral multiplets whose origin lies in the six-dimensional gauge sector,

$$
\text { Chiral multiplets at } n_{2}=0 \text { from the } 6 \text { d gauge vector: } \quad \mathbf{5}, \overline{\mathbf{5}} \text { of } \mathrm{SU}(5) \text {. }
$$

These fields will play an important role in the discussion of the phenomenology of the model in the next chapter. The term 'gauge-Higgs unification' describes a situation where these two fields are interpreted as the two Higgs multiplets of a supersymmetric SU(5) GUT at $n_{2}=0$. Note that they have four-dimensional zero modes which transform as weak doublets, so they may be suitable. If only one of them is associated with a Higgs multiplet, one refers to that as 'partial gauge-Higgs unification'. As we shall see, the interpretation of multiplets as Higgs or matter fields is a question of the choice of vacuum of the scalar potential. 


\begin{tabular}{c||c|c||c|c||c|c||c|c|c|c|c|c} 
Bulk & \multicolumn{2}{|c|}{$n_{2}=0$} & $n_{3}$ & $\gamma_{3}^{\prime}$ & $H_{L}$ & $H_{R}$ & $t_{1}$ & $t_{2}$ & $t_{3}$ & $t_{4}$ & $t_{5}$ & $t_{6}^{0}$ \\
\hline \hline$(\mathbb{1}, \mathbb{1}, \mathbb{1})$ & $(\mathbb{1}, \mathbb{1}, \mathbb{1})$ & $Y_{0}^{*}$ & 0 & $\frac{2}{3}$ &,,-+- &,,+-+ & 0 & $\frac{2}{3}$ & 0 & $\frac{2}{3}$ & 0 & 0 \\
\hline$(\mathbb{1}, \mathbb{1}, \mathbb{1})$ & $(\mathbb{1}, \mathbb{1}, \mathbb{1})$ & $\bar{Y}_{0}^{*}$ & 0 & $\frac{2}{3}$ &,+-+ &,,-+- & 0 & $\frac{2}{3}$ & 0 & $\frac{2}{3}$ & 0 & 0 \\
\hline \hline$(\mathbb{1}, \mathbb{1}, \mathbb{1})$ & $(\mathbb{1}, \mathbb{1}, \mathbb{1})$ & $Y_{1}^{*}$ & 1 & $\frac{2}{3}$ &,,-+- &,,+-+ & $-\frac{1}{2}$ & $\frac{1}{6}$ & 1 & $-\frac{1}{3}$ & -1 & 0 \\
\hline$(\mathbb{1}, \mathbb{1}, \mathbb{1})$ & $(\mathbb{1}, \mathbb{1}, \mathbb{1})$ & $\bar{Y}_{1}^{*}$ & 1 & $\frac{2}{3}$ &,+-+ &,,-+- & $-\frac{1}{2}$ & $\frac{1}{6}$ & 1 & $-\frac{1}{3}$ & -1 & 0 \\
\hline \hline$(\mathbb{1}, \mathbb{1}, \mathbb{1})$ & $(\mathbb{1}, \mathbb{1}, \mathbb{1})$ & $Y_{2}^{*}$ & 2 & $\frac{2}{3}$ &,,-+- &,,+-+ & $-\frac{1}{2}$ & $\frac{1}{6}$ & -1 & $-\frac{1}{3}$ & 1 & 0 \\
\hline$(\mathbb{1}, \mathbb{1}, \mathbb{1})$ & $(\mathbb{1}, \mathbb{1}, \mathbb{1})$ & $\bar{Y}_{2}^{*}$ & 2 & $\frac{2}{3}$ &,+-+ &,,-+- & $-\frac{1}{2}$ & $\frac{1}{6}$ & -1 & $-\frac{1}{3}$ & 1 & 0
\end{tabular}

Table 11: Projection of bulk hypermultiplets with oscillator numbers from twisted sectors $T_{2}$ and $T_{4}$ to the fixed points $n_{2}=0$. The singlets $Y_{n_{3}}^{*}$ have $\tilde{\mathbf{N}}=(0,1,0,0), \tilde{\mathbf{N}}^{*}=(0,0,0,0)$, the singlets $\bar{Y}_{n_{3}}^{*}$ have $\tilde{\mathbf{N}}=(0,0,0,0), \tilde{\mathbf{N}}^{*}=(1,0,0,0)$. The three parities for the chiral multiplet components $H_{L}, H_{R}$ correspond to $\gamma=0, \frac{1}{2}, 1$. The localization in the $\mathrm{G}_{2}$-plane is given by $n_{6}=0$ for $\gamma=0$, and $n_{6}=2$ otherwise.

\subsection{Anomaly cancelation}

The effective orbifold GUT field theory which was developed in the last sections is a complicated interacting theory. Additionally to the supergravity multiplet, the dilaton multiplet and the 76 vector multiplets, there are 320 hypermultiplets in the six-dimensional bulk, and 136 localized chiral multiplets. All fields participate in the gravitational interaction. Furthermore, the theory has local gauge symmetry which contains non-Abelian and Abelian factors, and the matter fields are in general charged under both the visible and the hidden sector U(1)'s.

The statement that the associated quantum theory is consistent up to its cutoff at the string scale is highly non-trivial. Here we explicitly check that all anomalies vanish or can be canceled by the Green-Schwarz mechanism [65]. This shows that the spectrum of the effective GUT model as derived from the heterotic string leads to a consistent gauge theory at the quantum level. Furthermore, an important outcome of the calculation is the presence of two distinct anomalous $\mathrm{U}(1)$ factors at the inequivalent fixed points. They exactly add to a single anomalous $\mathrm{U}(1)$ in the effective four-dimensional model, as it was found in [13].

Here we do not give a broad review of the topic of anomalies, but restrict to a brief introduction. The interested reader is referred to $[66,67]$ for more details and further references.

A quantum field theory with classical action $S$ and a symmetry transformation $\delta_{\epsilon}$ with parameter $\epsilon$ is anomalous if its one-loop effective action $\Gamma$ is not invariant under the symmetry,

$$
\text { Anomaly : } \quad \delta_{\epsilon} S=0, \quad \text { but } \mathcal{I}(\epsilon) \equiv \delta_{\epsilon} \Gamma \neq 0 .
$$

In gauge theories, the loss of gauge invariance due to an anomaly is accompanied by the loss of unitarity and renormalizability, and therefore the available description as a quantum field theory breaks down. It is thus a standard requirement for field theoretical model building 


\begin{tabular}{|c|c|c|c|c|c|c|c|c|c|c|c|c|}
\hline Bulk & \multicolumn{2}{|c|}{$n_{2}=0$} & $n_{3}$ & $\gamma_{3}^{\prime}$ & $H_{L}$ & $H_{R}$ & $t_{1}$ & $t_{2}$ & $t_{3}$ & $t_{4}$ & $t_{5}$ & $t_{6}^{0}$ \\
\hline \multirow[t]{2}{*}{$(6, \mathbb{1}, \mathbb{1})$} & $(5, \mathbb{1}, \mathbb{1})$ & & 0 & $\frac{1}{3}$ &,,-+- &,,+-+ & 0 & $-\frac{1}{3}$ & 1 & $\frac{2}{3}$ & 0 & -1 \\
\hline & $(\mathbb{1}, \mathbb{1}, \mathbb{1})$ & $X_{0}$ & 0 & $\frac{1}{3}$ &,+-+ &,,-+- & 0 & $-\frac{1}{3}$ & 1 & $\frac{2}{3}$ & 0 & 5 \\
\hline \multirow[t]{2}{*}{$(\overline{\mathbf{6}}, \mathbb{1}, \mathbb{1})$} & $(\overline{\mathbf{5}}, \mathbb{1}, \mathbb{1})$ & & 0 & 1 &,,-+- &,,+-+ & 0 & $-\frac{1}{3}$ & -1 & $\frac{2}{3}$ & 0 & 1 \\
\hline & $(\mathbb{1}, \mathbb{1}, \mathbb{1})$ & $\bar{X}_{0}$ & 0 & 1 &,+-+ &,,-+- & 0 & $-\frac{1}{3}$ & -1 & $\frac{2}{3}$ & 0 & -5 \\
\hline$(\mathbb{1}, \mathbb{1}, \mathbb{1})$ & $(\mathbb{1}, \mathbb{1}, \mathbb{1})$ & $Y_{0}$ & 0 & $\frac{2}{3}$ &,+-+ &,,-+- & 1 & $-\frac{1}{3}$ & 0 & $\frac{2}{3}$ & 0 & 0 \\
\hline$(\mathbb{1}, \mathbb{1}, \mathbb{1})$ & $(\mathbb{1}, \mathbb{1}, \mathbb{1})$ & $\bar{Y}_{0}$ & 0 & $\frac{2}{3}$ &,+-+ &,,-+- & -1 & $-\frac{1}{3}$ & 0 & $\frac{2}{3}$ & 0 & 0 \\
\hline$(\mathbb{1}, \mathbf{3}, \mathbb{1})$ & $(\mathbb{1}, \mathbf{3}, \mathbb{1})$ & & 0 & $\frac{1}{3}$ &,,-+- &,,+-+ & 0 & $\frac{2}{3}$ & 0 & $-\frac{1}{3}$ & 1 & 0 \\
\hline$(\mathbb{1}, \overline{\mathbf{3}}, \mathbb{1})$ & $(\mathbb{1}, \overline{\mathbf{3}}, \mathbb{1})$ & & 0 & 1 &,,-+- &,,+-+ & 0 & $\frac{2}{3}$ & 0 & $-\frac{1}{3}$ & -1 & 0 \\
\hline$(\mathbb{1}, \mathbb{1}, \mathbf{8})$ & $(\mathbb{1}, \mathbb{1}, \mathbf{8})$ & & 0 & $\frac{2}{3}$ &,,-+- &,,+-+ & 0 & $\frac{2}{3}$ & 0 & $-\frac{1}{3}$ & 0 & 0 \\
\hline \multirow[t]{2}{*}{$(6, \mathbb{1}, \mathbb{1})$} & $(5, \mathbb{1}, \mathbb{1})$ & & 1 & $\frac{1}{3}$ &,+-+ &,,-+- & 0 & $-\frac{1}{3}$ & -1 & $-\frac{1}{3}$ & -1 & -1 \\
\hline & $(\mathbb{1}, \mathbb{1}, \mathbb{1})$ & $X_{1}$ & 1 & $\frac{1}{3}$ &,,-+- &,,+-+ & 0 & $-\frac{1}{3}$ & -1 & $-\frac{1}{3}$ & -1 & 5 \\
\hline \multirow[t]{2}{*}{$(\overline{\mathbf{6}}, \mathbb{1}, \mathbb{1})$} & $(\overline{\mathbf{5}}, \mathbb{1}, \mathbb{1})$ & & 1 & 1 &,+-+ &,,-+- & $\frac{1}{2}$ & $\frac{1}{6}$ & 0 & $-\frac{1}{3}$ & -1 & 1 \\
\hline & $(\mathbb{1}, \mathbb{1}, \mathbb{1})$ & $\bar{X}_{1}$ & 1 & 1 &,,-+- &,,+-+ & $\frac{1}{2}$ & $\frac{1}{6}$ & 0 & $-\frac{1}{3}$ & -1 & -5 \\
\hline$(\mathbb{1}, \mathbb{1}, \mathbb{1})$ & $(\mathbb{1}, \mathbb{1}, \mathbb{1})$ & $Y_{1}$ & 1 & $\frac{2}{3}$ &,+-+ &,,-+- & 0 & $\frac{2}{3}$ & -2 & $-\frac{1}{3}$ & -1 & 0 \\
\hline$(\mathbb{1}, \mathbb{1}, \mathbb{1})$ & $(\mathbb{1}, \mathbb{1}, \mathbb{1})$ & $\bar{Y}_{1}$ & 1 & $\frac{2}{3}$ &,+-+ &,,-+- & $\frac{1}{2}$ & $-\frac{5}{6}$ & 1 & $-\frac{1}{3}$ & -1 & 0 \\
\hline$(\mathbb{1}, \mathbf{3}, \mathbb{1})$ & $(\mathbb{1}, \mathbf{3}, \mathbb{1})$ & & 1 & $\frac{1}{3}$ &,,-+- &,,+-+ & $-\frac{1}{2}$ & $\frac{1}{6}$ & 1 & $\frac{2}{3}$ & 0 & 0 \\
\hline$(\mathbb{1}, \overline{\mathbf{3}}, \mathbb{1})$ & $(\mathbb{1}, \overline{\mathbf{3}}, \mathbb{1})$ & & 1 & 1 &,,-+- &,,+-+ & $-\frac{1}{2}$ & $\frac{1}{6}$ & 1 & $-\frac{1}{3}$ & 1 & 0 \\
\hline$\left(\mathbb{1}, \mathbb{1}, \boldsymbol{8}_{s}\right)$ & $\left(\mathbb{1}, \mathbb{1}, \mathbf{8}_{s}\right)$ & & 1 & $\frac{2}{3}$ &,+-+ &,,-+- & $-\frac{1}{2}$ & $\frac{1}{6}$ & 1 & $\frac{1}{6}$ & $\frac{1}{2}$ & 0 \\
\hline \multirow[t]{2}{*}{$(6, \mathbb{1}, \mathbb{1})$} & $(5, \mathbb{1}, \mathbb{1})$ & & 2 & $\frac{1}{3}$ &,,-+- &,,+-+ & $\frac{1}{2}$ & $\frac{1}{6}$ & 0 & $-\frac{1}{3}$ & 1 & -1 \\
\hline & $(\mathbb{1}, \mathbb{1}, \mathbb{1})$ & $X_{2}$ & 2 & $\frac{1}{3}$ &,+-+ &,,-+- & $\frac{1}{2}$ & $\frac{1}{6}$ & 0 & $-\frac{1}{3}$ & 1 & 5 \\
\hline \multirow[t]{2}{*}{$(\overline{\mathbf{6}}, \mathbb{1}, \mathbb{1})$} & $(\overline{\mathbf{5}}, \mathbb{1}, \mathbb{1})$ & & 2 & 1 &,+-+ &,,-+- & 0 & $-\frac{1}{3}$ & 1 & $-\frac{1}{3}$ & 1 & 1 \\
\hline & $(\mathbb{1}, \mathbb{1}, \mathbb{1})$ & $\bar{X}_{2}$ & 2 & 1 &,,-+- &,,+-+ & 0 & $-\frac{1}{3}$ & 1 & $-\frac{1}{3}$ & 1 & -5 \\
\hline$(\mathbb{1}, \mathbb{1}, \mathbb{1})$ & $(\mathbb{1}, \mathbb{1}, \mathbb{1})$ & $Y_{2}$ & 2 & $\frac{2}{3}$ &,+-+ &,,-+- & 0 & $\frac{2}{3}$ & 2 & $-\frac{1}{3}$ & 1 & 0 \\
\hline$(\mathbb{1}, \mathbb{1}, \mathbb{1})$ & $(\mathbb{1}, \mathbb{1}, \mathbb{1})$ & $\bar{Y}_{2}$ & 2 & $\frac{2}{3}$ &,,-+- &,,+-+ & $\frac{1}{2}$ & $-\frac{5}{6}$ & -1 & $-\frac{1}{3}$ & 1 & 0 \\
\hline$(\mathbb{1}, \mathbf{3}, \mathbb{1})$ & $(\mathbb{1}, \mathbf{3}, \mathbb{1})$ & & 2 & $\frac{1}{3}$ &,+-+ &,,-+- & $-\frac{1}{2}$ & $\frac{1}{6}$ & -1 & $-\frac{1}{3}$ & -1 & 0 \\
\hline$(\mathbb{1}, \overline{\mathbf{3}}, \mathbb{1})$ & $(\mathbb{1}, \overline{\mathbf{3}}, \mathbb{1})$ & & 2 & 1 &,+-+ &,,-+- & $-\frac{1}{2}$ & $\frac{1}{6}$ & -1 & $\frac{2}{3}$ & 0 & 0 \\
\hline$\left(\mathbb{1}, \mathbb{1}, \mathbf{8}_{c}\right)$ & $\left(\mathbb{1}, \mathbb{1}, \mathbf{8}_{c}\right)$ & & 2 & $\frac{2}{3}$ &,,-+- &,,+-+ & $-\frac{1}{2}$ & $\frac{1}{6}$ & -1 & $\frac{1}{6}$ & $-\frac{1}{2}$ & 0 \\
\hline
\end{tabular}

Table 12: Projection of bulk hypermultiplets from twisted sectors $T_{2}$ and $T_{4}$ with vanishing oscillator numbers to the fixed points $n_{2}=0$. For excited states see Table 11 . The three parities for the chiral multiplet components $H_{L}, H_{R}$ correspond to $\gamma=0, \frac{1}{2}, 1$. $H_{L}$ is associated with $\mathbf{q}=\left(-\frac{1}{3},-\frac{2}{3}, 0,0\right),\left(\frac{1}{6},-\frac{1}{6}, \frac{1}{2},-\frac{1}{2}\right)$ and $H_{R}$ with $\mathbf{q}=\left(\frac{2}{3}, \frac{1}{3}, 0,0\right),\left(\frac{1}{6},-\frac{1}{6},-\frac{1}{2}, \frac{1}{2}\right)$. The localization in the $\mathrm{G}_{2}$-plane is given by $n_{6}=0$ for $\gamma=0$, and $n_{6}=2$ otherwise. 

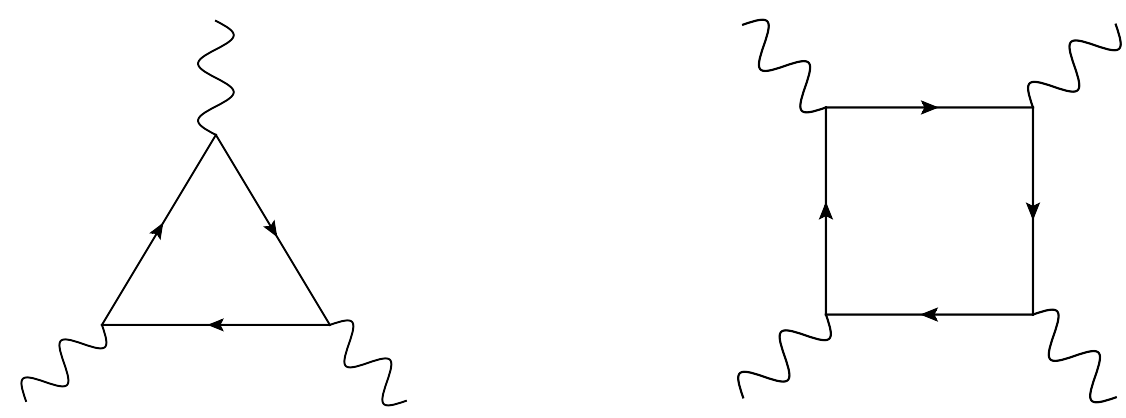

Figure 5: The one-loop diagrams which induce anomalies in four (left) and six (right) dimensions. Lines with arrows represent fermions. Wiggly lines represent gauge bosons or gravitons.

that the spectrum has to be anomaly-free. This requirement also applies to string theory in ten dimensions. String theories are as well anomaly free by construction, and since our effective model is derived from one of them we shall benefit from this fact also in six dimensions.

Anomalies are known and studied since the end 1960's, and they have had important impact on the understanding of the standard model. Pion to photon decays could only be consistently described after the discovery of the chiral anomaly [68], also other hadronic and semileptonic decays as well as meson masses [69] and the QCD U(1) problem [70] are related to anomalies. Note that these refer to global symmetries, such as the axial symmetry in QCD, and do not harm the quantum consistency of the standard model. With respect to its local symmetries, the latter is anomaly-free.

In general, anomalies can arise in any model with even dimensions. They are a consequence of the presence of Weyl fermions, which have a non-invariant measure of integration in the path integral [71]. Additionally, in $d=4 n+2$ dimensions gravitational anomalies may appear, related to local coordinate transformations. Their complete calculation was done in [72]. In six dimensions, we thus have to deal with both types of anomalies, and also combinations of gauge and gravitational anomalies appear, called mixed anomalies.

Anomalies in $d=2 n$ dimensions can be related to divergent one-loop Feynman diagrams with $n+1$ external gauge boson legs and chiral fermions running in the loop. In four dimensions the relevant diagram is the triangle diagram, in six dimensions it is the box diagram, as shown in Figure 5. These diagrams are divergent and the anomaly is related to the finite part of the corresponding expressions.

\subsubsection{The Stora-Zumino descent relations}

For local symmetries, the transformation parameter $\epsilon$ in (201) is space-time dependent. Furthermore, it takes values in the Lie-algebra of the symmetry group. Thus two successive transformations have to be compatible with the corresponding non-Abelian group 
structure:

$$
\left[\delta_{\epsilon_{1}}, \delta_{\epsilon_{2}}\right]=\delta_{\left[\epsilon_{1}, \epsilon_{2}\right]} .
$$

This basic requirement implies the Wess-Zumino consistency relations [69],

$$
\delta_{\epsilon_{1}} \mathcal{I}\left(\epsilon_{2}\right)-\delta_{\epsilon_{2}} \mathcal{I}\left(\epsilon_{1}\right)=\mathcal{I}\left(\left[\epsilon_{1}, \epsilon_{2}\right]\right),
$$

and an anomaly is called consistent if this relation holds. The general solution in even dimensions $d=2 n$ for an arbitrary local symmetry can be deduced from the Stora-Zumino descent relations [73], which will also be the main tool for the calculation of the anomalies in the six-dimensional orbifold model under investigation. They read ${ }^{18}$

$$
\mathcal{I}(\epsilon)=2 \pi i \int_{M_{2 n}} \Omega_{2 n}^{(1)}(\epsilon), \quad \mathrm{d} \Omega_{2 n}^{(1)}=\delta_{\epsilon} \Omega_{2 n+1}^{(0)}, \quad \mathrm{d} \Omega_{2 n+1}^{(0)}=\Omega_{2 n+2},
$$

and will be explained in the following.

The main observation is that they relate the anomaly $\mathcal{I}$ in $d=2 n$ dimensions to a $(2 n+2)$-form $\Omega_{2 n+2}$. This $(2 n+2)$-form is a gauge invariant function of the field strength $F$ and the curvature $R$. In fact, it describes the chiral anomaly in $2 n+2$ dimensions [74] and can be calculated by topological methods. This means that the quantity $\Omega_{2 n+2}(F, R)$ is known, and the appearing invariants can be evaluated for the given spectrum.

Chiral anomalies in $d=2 n+2=2 m$ dimensions arise from Dirac fermions $\psi$, in contrast to the local gauge and gravitational anomalies, that we are originally interested in. The latter rely on the presence of Weyl fermions. The chiral anomaly corresponds to global chiral transformations of the Dirac spinors, generated by the chirality matrix $\gamma_{2 m+1}$. It has the classically conserved current

$$
J_{2 m+1}^{M}=\bar{\psi}_{A} \gamma_{2 m+1} \gamma^{M} \psi^{A}
$$

where $M$ is an euclidean space-time index and $A$ labels the states of the gauge representation $\mathcal{R}$ of $\psi$. This current is not conserved in the quantum theory,

$$
\left\langle\partial_{M} J_{2 m+1}^{M}\right\rangle=2 i \int_{M_{2 m}} \Omega_{2 m}
$$

which is identical to the statement that the chiral symmetry is anomalous. The quantity on the right hand side can then be related to topological quantities of the gauge bundle and the tangent bundle, which are associated with gauge and gravitational interactions [72]. They are constructed from the Yang-Mills field strength and the Riemann curvature two-form,

$$
\begin{aligned}
& F=\frac{1}{2} F_{M N} \mathrm{~d} x^{M} \wedge \mathrm{d} x^{N}=\mathrm{d} A+A \wedge A, \\
& R=\frac{1}{2} R_{M N} \mathrm{~d} x^{M} \wedge \mathrm{d} x^{N}=\mathrm{d} \omega+\omega \wedge \omega,
\end{aligned}
$$

\footnotetext{
${ }^{18}$ The following results are obtained in Euclidean space. It is expected that they can be Wick-rotated to Minkowski space.
} 
where $A$ and $\omega$ denote the gauge and spin connection, respectively. Note that matrix indices were suppressed, $F_{M N i}{ }^{j}$ and $R_{M N a}{ }^{b}$, where $i, j$ label states of the gauge representation $\mathcal{R}$, and $a, b$ correspond to the vector representation of the Lorentz group in $2 m$ dimensions. For the case of spin $1 / 2$ the chiral anomaly is expressed by the product of the Chern character $\operatorname{ch}_{\mathcal{R}}(F)$ and the Dirac genus $\hat{A}(R)$. One can then obtain the result for $\Omega_{2 m}$ from a power series expansion:

$$
\begin{aligned}
\Omega_{2 m}= & \left.\operatorname{ch}_{\mathcal{R}}(F) \hat{A}(R)\right|_{2 m \text {-form component }} \\
\operatorname{ch}_{\mathcal{R}}(F)= & r+\frac{i}{2 \pi} \operatorname{tr}_{\mathcal{R}} F-\frac{1}{2(2 \pi)^{2}} \operatorname{tr}_{\mathcal{R}} F^{2} \\
& -\frac{i}{6(2 \pi)^{3}} \operatorname{tr}_{\mathcal{R}} F^{3}+\frac{1}{24(2 \pi)^{4}} \operatorname{tr}_{\mathcal{R}} F^{4}+\cdots, \\
\hat{A}(R)= & 1+\frac{1}{48(2 \pi)^{2}} \operatorname{tr} R^{2}+\frac{1}{4 !(2 \pi)^{4}}\left[\frac{1}{192}\left(\operatorname{tr} R^{2}\right)^{2}+\frac{1}{240} \operatorname{tr} R^{4}\right]+\cdots
\end{aligned}
$$

Here $\operatorname{tr}_{\mathcal{R}}$ denotes the trace evaluated in the representation $\mathcal{R}$ of dimension $r$, and wedge products are understood. Similar expressions arise for fermions of higher spins. By the use of the Stora-Zumino relations (204) one can now calculate the gauge and gravitational anomalies in $d=2 n$ dimensions from the above result for the chiral anomaly in $2 m=2 n+2$ dimensions. However, the analogy between the two anomalies holds up to a minus sign, which is related to the chirality of the fermions on the side of the gauge and gravitational anomaly. Furthermore, the total $(2 n+2)$-form is the sum of the contributions of all fermions in the spectrum. We will now evaluate the corresponding anomalies for the six-dimensional model of interest.

\subsubsection{The bulk anomaly polynomial}

In six dimensions, the gauge and gravity anomalies (cf. $[35,75]$ ) can be deduced from an eight-form, studied in [76-78]. It is convenient to introduce the anomaly polynomial $I_{8}$,

$$
I_{8} \equiv-16(2 \pi)^{3}\left(2 \pi \Omega_{8}\right)
$$

since the factor $1 / 16(2 \pi)^{3}$ appears throughout the calculation. The result (209) corresponds to the contribution of a single fermion of spin $1 / 2$. In total, the anomaly polynomial arises from the sum of all fermionic contributions,

$$
I_{8}=\underbrace{I_{(3 / 2)}(R)}_{\text {gravitino }}-\underbrace{I_{(1 / 2)}(R)}_{\text {dilatino }}+\underbrace{I_{(1 / 2)}(F, R)}_{\text {gauginos }}-\sum_{i} s^{i} \underbrace{I_{(1 / 2)}^{i}(F, R)}_{\text {hyperinos }},
$$

where $s^{i}$ denotes the multiplicity of the representation $\mathcal{R}_{i}$ of the bulk hypermultiplets. Note that the effect from the self-dual antisymmetric two-form $B_{M N}^{+}$is canceled by the terms due to its anti-self dual partner, $B_{M N}^{-}$, so they do not appear in the anomaly polynomial. The different signs for the contributions of the dilatino and the hyperinos as compared to 


\begin{tabular}{|c|c|c|c|}
\hline $\operatorname{tr}_{\mathcal{R}} F^{n}$ & & $\mathrm{SU}(N)$ & $\mathrm{SO}(N)$ \\
\hline $\operatorname{tr}_{\mathcal{A}} F^{2}$ & \multicolumn{2}{|r|}{$2 N \operatorname{tr} F^{2}$} & $(N-2) \operatorname{tr} F^{2}$ \\
\hline $\operatorname{tr}_{\mathcal{A}} F^{4}$ & $\begin{array}{l}N=2,3 \\
N>3\end{array}$ & $\begin{array}{l}\frac{1}{2}\left(\operatorname{tr} F^{2}\right)^{2} \\
2 N \operatorname{tr} F^{4}+6\left(\operatorname{tr} F^{2}\right)^{2}\end{array}$ & $(N-8) \operatorname{tr} F^{4}+3\left(\operatorname{tr} F^{2}\right)^{2}$ \\
\hline $\operatorname{tr}_{\mathcal{S}} F^{2}$ & & & $2^{(N-8) / 2} \operatorname{tr} F^{2}$ \\
\hline $\operatorname{tr}_{\mathcal{S}} F^{4}$ & & & $-2^{(N-10) / 2} \operatorname{tr} F^{4}+3\left(2^{(N-14) / 2}\right)\left(\operatorname{tr} F^{2}\right)^{2}$ \\
\hline $\operatorname{tr}_{a^{i j}} F^{3}$ & $N>2$ & $(N-4) \operatorname{tr} F^{3}$ & \\
\hline $\operatorname{tr}_{\mathrm{a}^{i j k}} F^{2}$ & & $\frac{1}{2}\left(N^{2}-5 N+6\right) \operatorname{tr} F^{2}$ & \\
\hline $\operatorname{tr}_{\mathrm{a}^{i j k}} F^{3}$ & & $\frac{1}{2}\left(N^{2}-9 N+18\right) \operatorname{tr} F^{3}$ & \\
\hline $\operatorname{tr}_{\mathrm{a}^{i j k}} F^{4}$ & $\frac{1}{2}\left(N^{2}-1\right)$ & $N+54) \operatorname{tr} F^{4}+(3 N-12)\left(\operatorname{tr} F^{2}\right)^{2}$ & \\
\hline
\end{tabular}

Table 13: A collection of trace identities for representations $\mathcal{R}$ of $\mathrm{SU}(N)$ and $\mathrm{SO}(N)$. Here $\mathcal{A}$ and $\mathcal{S}$ denote adjoint and spinor representations and $a^{i j k}$ the third rank antisymmetric tensor representation, respectively. The trace in the fundamental representation is denoted as tr.

the gravitino and the gauginos stem from the fact that they have opposite $6 \mathrm{~d}$ chirality, cf. (151), (157), (175).

For the spin $3 / 2$ gravitino, the contribution to the anomaly polynomial is [72]

$$
I_{(3 / 2)}(R)=-\frac{16}{4 !}\left[\frac{245}{240} \operatorname{tr} R^{4}-\frac{43}{192}\left(\operatorname{tr} R^{2}\right)^{2}\right],
$$

and therefore the sum of all purely curvature dependent terms is

$$
I_{\text {grav }}(R)=-\frac{16}{4 ! 240} \underbrace{(244+y-s)}_{0} \operatorname{tr} R^{4}-\frac{16}{4 ! 192}(-44+y-s)\left(\operatorname{tr} R^{2}\right)^{2},
$$

where $y=76$ counts the total number of gauginos, and $s=320$ the number of hyperinos in the six-dimensional bulk. This demonstrates that the gravitational $R^{4}$ anomaly cancels in the model. This is a necessary requirement, since there are no alternative mechanisms to the direct cancelation, for example by additional counter terms. Anomalies with that property are called 'irreducible', in contrast to reducible ones which can for example be canceled by the Green-Schwarz mechanism, which will be discussed later.

All remaining terms in the anomaly polynomial involve traces over the representation indices of powers of the field strength two-forms. Recall that the bulk gauge group is given by (163). Let $A$ and $u$ label the non-Abelian and the Abelian gauge factors, respectively,

$$
A=\mathrm{SU}(6), \mathrm{SU}(3), \mathrm{SO}(8), \quad u=1, \ldots, 5 .
$$

One can then convert all traces $\operatorname{tr}_{\mathcal{R}} F^{n}$ into expressions proportional to the trace in the fundamental representation, denoted as tr. This is summarized in Table 13 for $\mathrm{SU}(N)$ and $\mathrm{SO}(N)$, which collects trace identities as given in $[77,78]$. 
According to (209a), the anomaly polynomial for the spin $1 / 2$ fermions is given by all combinations of terms from (209b) and (209c) which lead to an eight form. We can collect them into expressions $I_{(1 / 2)}\left(F^{n}, R^{m}\right)$, where $n+m=4$ :

$$
\begin{aligned}
I_{(1 / 2)}\left(F^{4}, R^{0}\right)= & -\frac{2}{3} \underbrace{\left(12+6 s_{\mathbf{2 0}}-s_{\mathbf{6}}-s_{\overline{\mathbf{6}}}\right)}_{0} \operatorname{tr} F_{\mathrm{SU}(6)}^{4}-\frac{2}{3} \underbrace{\left(\frac{1}{2} s_{\boldsymbol{8}_{s}}+\frac{1}{2} s_{\boldsymbol{8}_{c}}-s_{\mathbf{8}}\right)}_{0} \operatorname{tr} F_{\mathrm{SO}(8)}^{4} \\
& -\frac{2}{3} \underbrace{\left(6-6 s_{\mathbf{2 0}}\right)}_{0}\left(\operatorname{tr} F_{\mathrm{SU}(6)}^{2}\right)^{2}-\frac{2}{3} \underbrace{\left(3-\frac{3}{8} s_{\boldsymbol{8}_{s}}-\frac{3}{8} s_{\boldsymbol{8}_{c}}\right)}_{0}\left(\operatorname{tr} F_{\mathrm{SO}(8)}^{2}\right)^{2} \\
& +I_{(1 / 2)}^{\mathrm{Abelian}_{(1 / 2)}\left(F^{4}, R^{0}\right)} \\
I_{(1 / 2)}\left(F^{2}, R^{2}\right)= & \frac{1}{6}[\underbrace{\left(12-6 s_{\mathbf{2 0}}-s_{\mathbf{6}}-s_{\overline{\mathbf{6}}}\right)}_{-12} \operatorname{tr} F_{\mathrm{SU}(6)}^{2}+\underbrace{\left(6-s_{\mathbf{3}}-s_{\overline{\mathbf{3}}}\right)}_{-12} \operatorname{tr} F_{\mathrm{SU}(3)}^{2} \\
& +I_{(1 / 2)}^{\mathrm{Abelian}\left(F^{2}, R^{2}\right) .}
\end{aligned}
$$

The number of hyperinos in the representation $\mathcal{R}$ is denoted as $s_{\mathcal{R}}$. From Tables 6 and 7 one can read off

$$
s_{\mathbf{2 0}}=1, \quad s_{\mathbf{6}}=s_{\overline{\mathbf{6}}}=9, \quad s_{\mathbf{3}}=s_{\overline{\mathbf{3}}}=9, \quad s_{\mathbf{8}}=s_{\boldsymbol{8}_{s}}=s_{\boldsymbol{8}_{c}}=4,
$$

which implies the numerous cancelations denoted above. They are necessary for a consistent model, since the corresponding anomalies are irreducible.

What remains to be studied are the contributions to the anomaly polynomial which involve $\mathrm{U}(1)$ factors, denoted as $I_{(1 / 2)}^{\text {Abelian }}\left(F^{4}, R^{0}\right)$ and $I_{(1 / 2)}^{\text {Abelian }}\left(F^{2}, R^{2}\right)$ above. They can be parametrized by constants $a_{A}^{u}, b_{A}^{u v}, c^{u v w x}, d^{u v}$,

$$
\begin{aligned}
I_{(1 / 2)}^{\text {Abelian }}\left(F^{4}, R^{0}\right)= & \frac{8}{3} \sum_{A} \sum_{u=1}^{5} a_{A}^{u}\left(\operatorname{tr} F_{A}^{3}\right) \hat{F}_{u}+4 \sum_{A} \sum_{u, v=1}^{5} b_{A}^{u v}\left(\operatorname{tr} F_{A}^{2}\right) \hat{F}_{u} \hat{F}_{v} \\
& +\frac{2}{3} \sum_{u, v, w, x=1}^{5} c^{u v w z} \hat{F}_{u} \hat{F}_{v} \hat{F}_{w} \hat{F}_{x} \\
I_{(1 / 2)}^{\text {Abelian }}\left(F^{2}, R^{2}\right)= & -\frac{1}{6}\left(\operatorname{tr} R^{2}\right) \sum_{u, v=1}^{5} d^{u v} \hat{F}_{u} \hat{F}_{v}
\end{aligned}
$$

where $\hat{F}_{u}=\mathrm{d} \hat{A}_{u}+\hat{A}_{u}^{2}$ is the field strength two-form which is associated with the canonically normalized generator $\hat{t}_{u}$,

$$
\hat{F}_{u}, \hat{A}_{u}: \quad \hat{t}_{u} \equiv \frac{t_{u}}{\sqrt{2}\left|t_{u}\right|} .
$$


In the literature, to our knowledge only cases with a single Abelian factor were studied (for example in [77]). However, the bulk spectrum of the orbifold model derived before has five $\mathrm{U}(1)$ factors, which leads to a complication of the analysis. Especially the cancelation of the related anomalies by the Green-Schwarz mechanism is a non-trivial check of the consistency of the model, as will be discussed later. Due to its technical nature we evaluate the (partly) Abelian traces in Appendix C.1, and directly state the result:

$$
\begin{aligned}
a_{\mathrm{SU}(6)}^{u} & =a_{\mathrm{SU}(3)}^{u}=a_{\mathrm{SO}(8)}^{u}=0, \\
b_{\mathrm{SU}(6)}^{u v} & =b_{\mathrm{SU}(3)}^{u v}=2 b_{\mathrm{SO}(8)}^{u v}=\frac{1}{2} \beta^{u v}, \\
c^{u v w x} & =\frac{3}{2|\sigma(u, v, w, x)|}\left(\delta^{u v} \beta^{w x}+\text { permutations }\right), \\
d^{u v} & =6\left(\beta^{u v}+\delta^{u v}\right) .
\end{aligned}
$$

Here $|\sigma(u, v, w, x)|$ is a symmetry factor, which is one if all its variables are distinct, and equal to the number of possibilities to assign the identical labels to the four $F$ 's otherwise. Furthermore, $\beta^{u v}$ is a symmetric $5 \times 5$ matrix,

$$
\left(\beta^{u v}\right)=\left(\begin{array}{ccccc}
3 & -1 & 0 & -1 & 0 \\
-1 & 3 & 0 & -1 & 0 \\
0 & 0 & 2 & 0 & \sqrt{2} \\
-1 & -1 & 0 & 4 & 0 \\
0 & -0 & \sqrt{2} & 0 & 4
\end{array}\right)
$$

demonstrating that the model has non-trivial (partly) Abelian anomalies in the bulk. However, as we will demonstrate in the next section, all anomalies can be canceled by a classical term in the action.

Summarizing the results (213), (216) and (218) with (220), the total remaining anomaly polynomial in the six-dimensional bulk is

$$
\begin{aligned}
I_{8}= & \left(\operatorname{tr} R^{2}\right)^{2}-\left(2 \operatorname{tr} F_{\mathrm{SU}(6)}^{2}+2 \operatorname{tr} F_{\mathrm{SU}(3)}^{2}+\operatorname{tr} F_{\mathrm{SO}(8)}^{2}+\hat{\mathbf{F}} \cdot \beta \cdot \hat{\mathbf{F}}+\hat{\mathbf{F}}^{2}\right) \operatorname{tr} R^{2} \\
& +\left(2 \operatorname{tr} F_{\mathrm{SU}(6)}^{2}+2 \operatorname{tr} F_{\mathrm{SU}(3)}^{2}+\operatorname{tr} F_{\mathrm{SO}(8)}^{2}+\hat{\mathbf{F}}^{2}\right) \hat{\mathbf{F}} \cdot \beta \cdot \hat{\mathbf{F}}
\end{aligned}
$$

with vector notation $\hat{\mathbf{F}}=\left(\hat{F}_{1}, \ldots, \hat{F}_{5}\right)$ for the Abelian field strength two-forms.

\subsubsection{Anomaly polynomials at fixed points}

Similarly to the bulk case, we can now evaluate the anomaly polynomials $I_{n_{2}, 6}$ which are related to the spectra at the fixed points, labeled by $n_{2}=0,1$. In that case two dimensions are fixed. Hence the localized fields only propagate in four dimensions and the anomaly polynomial is a six-form,

$$
I_{n_{2}, 6} \equiv-48(2 \pi)^{3} i \Omega_{6},
$$


where $\Omega_{6}$ follows from (209).

Following the same program as before, it can be parametrized as

$$
I_{6}=\operatorname{tr} R^{2} \sum_{v} \tilde{a}^{v} \hat{F}_{v}-8 \sum_{B} \tilde{b}_{B} \operatorname{tr} F_{B}^{3}-24 \sum_{B, v} \tilde{c}_{B}^{v} \operatorname{tr} F_{B}^{2} \hat{F}_{v}-8 \sum_{v, w, x} \tilde{d}_{B}^{v w x} \hat{F}_{v} \hat{F}_{w} \hat{F}_{x}
$$

where $\tilde{a}^{v}, \tilde{b}_{B}, \tilde{c}_{B}^{v}, \tilde{d}^{v w x}$ are coefficients, and $B$ and $v, w, x$ label the non-Abelian and the Abelian factors at the fixed point, respectively,

$$
B=\left\{\begin{array}{ll}
\mathrm{SU}(5), \mathrm{SU}(3), \mathrm{SO}(8), & \text { if } n_{2}=0, \\
\mathrm{SU}(2), \mathrm{SU}(4), \mathrm{SU}(2)^{\prime}, \mathrm{SU}(4)^{\prime}, & \text { if } n_{2}=1,
\end{array} \quad v= \begin{cases}1, \ldots, 6, & \text { if } n_{2}=0 \\
1, \ldots, 8, & \text { if } n_{2}=1\end{cases}\right.
$$

The coefficients in (224) are determined in Appendix C.2, with results

$$
\begin{array}{cl}
n_{2}=0: \quad I_{6}=\left(\operatorname{tr} R^{2}-2 \operatorname{tr} F_{\mathrm{SU}(5)}-2 \operatorname{tr} F_{\mathrm{SU}(3)}-\operatorname{tr} F_{\mathrm{SO}(8)}-\hat{\mathbf{F}}^{2}\right) 2 \sqrt{37} \hat{F}_{\mathrm{an}}^{0}, \\
n_{2}=1: \quad I_{6}=\left(\operatorname{tr} R^{2}-2 \operatorname{tr} F_{\mathrm{SU}(2)}-2 \operatorname{tr} F_{\mathrm{SU}(4)}\right. \\
\left.-2 \operatorname{tr} F_{\mathrm{SU}(2)^{\prime}}-2 \operatorname{tr} F_{\mathrm{SU}(4)^{\prime}}-\hat{\mathbf{F}}^{2}\right) 2 \sqrt{10} \hat{F}_{\mathrm{an}}^{1},
\end{array}
$$

where $\hat{F}_{\text {an }}^{0}$ and $\hat{F}_{\text {an }}^{0}$ denote field strength two-forms associated with the canonically normalized generators $\hat{t}_{\mathrm{an}}^{0}$ and $\hat{t}_{\mathrm{an}}^{1}$, whose non-canonically normalized versions can be found in Table 4. They are linear combinations of the local U(1) factors,

$$
\begin{array}{ll}
t_{\mathrm{an}}^{0} \equiv-4 t_{2}+5 t_{4}-t_{5}+t_{6}^{0}, & \sqrt{2}\left|t_{\mathrm{an}}^{0}\right|=2 \sqrt{37}, \\
t_{\mathrm{an}}^{1} \equiv 3 t_{1}-t_{2}+t_{3}-4 t_{4}+4 t_{5}, & \sqrt{2}\left|t_{\mathrm{an}}^{1}\right|=4 \sqrt{10} .
\end{array}
$$

All generators of the Abelian groups in (226) and (227) are chosen orthogonal to each other, furthermore the generators $\hat{t}_{\text {an }}^{0}$ and $\hat{t}_{\text {an }}^{1}$ correspond to one of the indices $v$ and the corresponding contributions also appear inside the bracket.

The above vectors $t_{\mathrm{an}}^{0}$ and $t_{\mathrm{an}}^{1}$ are the generators of the local anomalous $\mathrm{U}(1)$ 's at the inequivalent fixed points $n_{2}=0$ and $n_{2}=1$. We stress that they are neither collinear nor orthogonal. It is an interesting observation that the model provides two different anomalous $\mathrm{U}(1)$ factors, localized at different fixed points, since it is a well-known fact that there can be at most one anomalous $\mathrm{U}(1)$ in the four-dimensional low-energy theory, after compactification of six dimensions of heterotic string theory. However, this is not contradicted by our findings. Summing over the zero modes, one finds that there is only one anomalous $\mathrm{U}(1)$ in the effective four-dimensional theory, with generator [13]

$$
t_{\mathrm{an}}^{4 \mathrm{~d}}=\frac{1}{3}\left(t_{\mathrm{an}}^{0}+\frac{1}{2} t_{\mathrm{an}}^{1}\right)
$$

It is given by the sum of the localized anomalous generators, weighted by the size of the anomalous traces. 


\subsubsection{Green-Schwarz anomaly cancelation in six dimensions}

The Green-Schwarz mechanism originally refers to string derived supergravities in ten dimensions [65]. It states that models with an anomalous spectrum can be anomaly free, due to the variation of counter terms in the action which are related to an antisymmetric tensor field. This is a remarkable statement, since it means that a one-loop quantum effect is balanced by a classical term in the action. The six-dimensional version of the mechanism is studied in $[67,77,79]$. Here we closely follow [66], for the special case of six dimensions.

In our model, the antisymmetric two-form is called $B_{2}$ and arises from the gravitational sector, cf. (152). Its kinetic term is determined by the three-form field strength

$$
H_{3}=\mathrm{d} B_{2}+\sqrt{2 \pi} \xi X_{3}^{(0)},
$$

where $\xi$ is a dimensionless parameter. The introduction of the three-form $X_{3}^{(0)}$ is necessary if $B_{2}$ transforms inhomogeneously under gauge transformations ${ }^{19}$,

$$
\delta_{\epsilon} B_{2}=-\sqrt{2 \pi} \xi X_{2}^{(1)}, \quad \mathrm{d} X_{2}^{(1)}=\delta_{\epsilon} X_{3}^{(0)}, \quad \mathrm{d} X_{3}^{(0)}=X_{4} .
$$

Here $X_{4}$ was introduced since it will appear in calculations soon. Thus the extra term in the definition of the field strength $H_{3}$ in (231) is responsible for invariance of the latter under local symmetry transformations.

The Green-Schwarz action in six dimensions is

$$
S=\int_{M_{6}}\left[\frac{1}{2}\left|H_{3}\right|^{2}+i \frac{\sqrt{2 \pi}}{\xi} B_{2} Y_{4}-2 \pi i\left(\frac{1}{2}+\alpha\right) X_{3}^{(0)} Y_{3}^{(0)}\right],
$$

where $\alpha, \xi$ are parameters and $Y_{4}$ is a four-form, related to $Y_{3}^{(0)}$ and another two-form $Y_{2}^{(1)}$ by

$$
\mathrm{d} Y_{3}^{(0)}=Y_{4}, \quad \delta_{\epsilon} Y_{3}^{(0)}=\mathrm{d} Y_{2}^{(1)} .
$$

The above action is not invariant under gauge transformations. One can calculate the variation as

$$
\begin{aligned}
\delta_{\epsilon} S & =-2 \pi i \int_{M_{6}}\left[X_{2}^{(1)} Y_{4}+\left(\frac{1}{2}+\alpha\right) \mathrm{d} X_{2}^{(1)} Y_{3}^{(0)}+\left(\frac{1}{2}+\alpha\right) X_{3}^{(0)} \mathrm{d} Y_{2}^{(1)}\right] \\
& =-2 \pi i \int_{M_{6}}\left[X_{2}^{(1)} Y_{4}-\left(\frac{1}{2}+\alpha\right) X_{2}^{(1)} Y_{4}+\left(\frac{1}{2}+\alpha\right) X_{4} Y_{2}^{(1)}\right] \\
& =-2 \pi i \int_{M_{6}}\left[\left(\frac{1}{2}-\alpha\right) X_{2}^{(1)} Y_{4}+\left(\frac{1}{2}+\alpha\right) X_{4} Y_{2}^{(1)}\right] \equiv-2 \pi i \int_{M_{6}} \Omega_{6}^{(1)},
\end{aligned}
$$

where partial integration was used. The action (233) can thus cancel the anomalous variation of the quantum contribution to the effective action $\Gamma$, if the anomaly is described by $\mathcal{I}=2 \pi i \int \Omega_{6}^{(1)}$, where $\Omega_{6}^{(1)}$ takes the form which appears in the bracket.

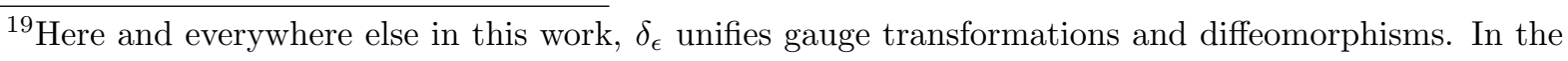
following we refer to the combined transformations as gauge transformations.
} 
This is the case if it descents from an eight form which factorizes into $X_{4}$ and $Y_{4}$,

$$
\Omega_{8}=X_{4} Y_{4} \quad \Rightarrow \quad \Omega_{6}^{(1)}=\left(\frac{1}{2}-\alpha\right) X_{2}^{(1)} Y_{4}+\left(\frac{1}{2}+\alpha\right) X_{4} Y_{2}^{(1)},
$$

where $\alpha$ is a free parameter and all field strengths are treated symmetrically.

Now reconsider the anomaly polynomial (222), which we found for the bulk theory in our model. It can be factorized as

$$
I_{8}=\left(\operatorname{tr} R^{2}-2 \operatorname{tr} F_{\mathrm{SU}(6)}^{2}-2 \operatorname{tr} F_{\mathrm{SU}(3)}^{2}-\operatorname{tr} F_{\mathrm{SO}(8)}^{2}-\hat{\mathbf{F}}^{2}\right)\left(\operatorname{tr} R^{2}-\hat{\mathbf{F}} \cdot \beta \cdot \hat{\mathbf{F}}\right),
$$

and thus we have shown that all anomalies either cancel among themselves, or are canceled by the variation of the Green-Schwarz action (233).

In fact, the coefficients $\alpha_{A}$ in the first bracket are generic and related to the dual Coxeter number of the gauge group [77],

$$
\alpha_{\mathrm{SU}(N)}=2, \quad \alpha_{\mathrm{SO}(N)}=1, \quad \alpha_{\mathrm{U}(1)}=1,
$$

where the latter corresponds to canonical normalization of the U(1) generator, as in (219). The second factor in (237) carries model dependent information, condensed into the matrix $\beta$. Note that the identification of the factors $X_{4}, Y_{4}$ also determines the classical action (233) of the antisymmetric tensor field $B_{2}$, as well as its gauge transformation (232), up to two free constants $\alpha, \xi$.

\subsubsection{Green-Schwarz anomaly cancelation on the orbifold}

So far we have demonstrated that the bulk theory of the effective orbifold GUT field theory is a consistent gauge theory with respect to quantum fluctuations. However, at the fixed points additional matter is generated, originating from twisted sectors, and the local consistency of the spectrum remains to be checked.

The Green-Schwarz action (233) can be extended to include fixed points $z_{f}^{3}$, associated with delta-functions $\delta_{2}^{f} \equiv \delta\left(x_{5}-x_{5}^{f}\right) \delta\left(x_{6}-x_{6}^{f}\right) \mathrm{d} x^{5} \wedge \mathrm{d} x^{6}$ and local two-forms $Y_{2}^{f}$ [66]:

$$
\begin{aligned}
S=\int_{M_{6}} & {\left[\frac{1}{2}\left|H_{3}\right|^{2}+i \frac{\sqrt{2 \pi}}{\xi} B_{2}\left(Y_{4}+\sum_{f} \delta_{2}^{f} Y_{2}^{f}\right)\right.} \\
& \left.-2 \pi i\left(\frac{1}{2}+\alpha\right) X_{3}^{(0)}\left(Y_{3}^{(0)}+\sum_{f} \delta_{2}^{f} Y_{1}^{f(0)}\right)\right] .
\end{aligned}
$$

Here $\xi$ and $\alpha$ are two dimensionless parameters which may be chosen arbitrarily without affecting the anomaly cancelation mechanism. The variation of this action contains local contributions,

$$
\delta_{\epsilon} S=-2 \pi i \int_{M_{6}}\left[\Omega_{6}^{(1)}+\sum_{f} \delta_{2}^{f} \Omega_{4}^{f(1)}\right]
$$


where $\Omega_{6}^{(1)}$ was defined before in (235), and

$$
\Omega_{4}^{f(1)}=\left(\frac{1}{2}-\alpha\right) X_{2}^{(1)} Y_{2}^{f}+\left(\frac{1}{2}+\alpha\right) X_{4} Y_{0}^{f(1)} .
$$

The action (239) is thus suitable for the cancelation of reducible anomalies with an anomaly polynomial of the form

$$
I_{8}=X_{4} Y_{4}+\sum_{f} \delta_{2}^{f} X_{4} Y_{2}^{f}
$$

The factorization of the bulk anomaly polynomial was discussed in the previous section, with

$$
X_{4}=\operatorname{tr} R^{2}-2 \operatorname{tr} F_{\mathrm{SU}(6)}^{2}-2 \operatorname{tr} F_{\mathrm{SU}(3)}^{2}-\operatorname{tr} F_{\mathrm{SO}(8)}^{2}-\hat{\mathbf{F}}^{2} .
$$

At the fixed points some of the field strength forms vanish due to the boundary conditions of the fields. The localized anomalies from (226) and (227) thus only contain a projection of this factor,

$$
\begin{array}{cc}
n_{2}=0: & I_{6}=\left(\operatorname{tr} R^{2}-2 \operatorname{tr} F_{\mathrm{SU}(5)}-2 \operatorname{tr} F_{\mathrm{SU}(3)}-\operatorname{tr} F_{\mathrm{SO}(8)}-\hat{\mathbf{F}}^{2}\right) 2 \sqrt{37} \hat{F}_{\mathrm{an}}^{0}, \\
n_{2}=1: \quad I_{6}=\left(\operatorname{tr} R^{2}-2 \operatorname{tr} F_{\mathrm{SU}(2)}-2 \operatorname{tr} F_{\mathrm{SU}(4)}\right. \\
\left.\quad-2 \operatorname{tr} F_{\mathrm{SU}(2)^{\prime}}-2 \operatorname{tr} F_{\mathrm{SU}(4)^{\prime}}-\hat{\mathbf{F}}^{2}\right) 2 \sqrt{10} \hat{F}_{\mathrm{an}}^{1} .
\end{array}
$$

Note that the definitions of the U(1) factors are different here, as discussed below (227).

We have thus shown that all anomalies of the model cancel. Either among the fields of the spectrum, in the case of irreducible anomalies, or by classical counter terms, in the case of reducible anomalies. With respect to quantum corrections, the model is a consistent gauge field theory on an orbifold in six dimensions.

\section{Vacua and phenomenology}

The six-dimensional GUT model, as discussed so far, does not describe the visible world. It is obvious that it contains many exotic particles, which are inconsistent with observations. Furthermore, even though all anomalies are canceled by a bulk field, the non-vanishing traces of anomalous $\mathrm{U}(1)$ factors imply that supersymmetry is broken radiatively by induced Fayet-Iliopoulos $D$-terms at a large scale [46], if the vacuum expectation values of all fields are zero. What was described in the last chapter thus cannot be a stable vacuum solution of a compactification of perturbative string theory, which should be supersymmetric. One rather expects the presence of a tachyonic direction in the effective potential which drives the system towards its physical vacuum.

The main point of this chapter is to establish a relation between the unbroken symmetries in this new vacuum, which are mainly discrete symmetries, and the interactions in the 
effective GUT theory. The latter arise from higher-dimensional operators, after integrating out singlet fields. The symmetries will guide us to an algorithm which determines vacuum configurations with the property that phenomenologically disfavored couplings are forbidden to all orders in the singlet fields, as long as supersymmetry is unbroken. This result and its application to the $\mu$-term [17] is reviewed in Sections 4.5 and 4.6. Furthermore, Section 4.7 discusses the reverse argument, namely the generation of the superpotential terms to all orders in the singlets, in a given vacuum.

The abovementioned problems, the presence of exotics and supersymmetry breaking Fayet-Iliopoulos terms $\xi$, are by no means specific to our model. They generically arise in orbifold compactifications of the heterotic string. The usual approach to overcoming them is to assume expectation values of singlet fields, thereby guessing the location of the supersymmetric vacuum in the potential,

$$
\begin{array}{rll}
\text { False vacuum: } & \left\langle s_{i}\right\rangle=0 \quad \text { for all singlets } s_{i}, & D \sim \xi \neq 0, \\
\text { Physical vacuum: } & \left\langle s_{i}\right\rangle \neq 0 \quad \text { for some singlets } s_{i}, & D=0,
\end{array}
$$

However, due to the size of the supersymmetry breaking $D$-term this new vacuum is not necessarily nearby the origin, and it is not clear that the findings from the false vacuum can be extrapolated. This worry is linked to the observation that vacuum expectation values of localized fields imply a resolution of the orbifold singularities (cf. [40,51-56]). The orbifold result, shifted to a vacuum remote from the origin, may then correspond to an effective description of a compactification on a smooth manifold. Here we shall take the optimistic viewpoint that orbifolds, even after the transition to the supersymmetric vacuum, are a first-order approximation to the results one would obtain in a fully dynamical calculation, if the latter was doable.

We therefore now turn to the study of possibly interesting physical vacua of the model at hand, and return to the issue of contact with smooth geometries in the following chapter. Here our analysis is guided by phenomenological requirements. We thus hope to reveal some of its mechanisms which determine the role of the various fields in the spectrum. We furthermore hope to understand the presence or absence of couplings among the fields of the local GUT theory, and consequently also of the low-energy zero modes.

\subsection{The SU(5) GUT superpotential}

The effective orbifold GUT in six dimensions which was derived in the previous chapter contains fixed points with SU(5) gauge symmetry, localized and bulk-projected matter. What we are aiming at in this chapter is the construction of a viable local SU(5) GUT theory at these fixed points. For that it is instructive to recall the ingredients and interactions of standard SU(5) GUTs.

The group SU(5) was the first simple group which was suggested for grand unification ${ }^{20}$, by Georgi and Glashow in 1974 [24], and it is somehow the simplest choice. In the following

\footnotetext{
${ }^{20}$ The first proposal for extended gauge symmetry by Pati and Salam was based on a non-simple Lie group [80], corresponding to three independent gauge couplings.
} 
we will need its supersymmetric version, therefore we associate every gauge multiplet with a chiral multiplet of $\mathcal{N}=1$ supersymmetry in four dimensions.

One matter generation consists of one antisymmetric tensor representation of rank two and one anti-fundamental representation,

$$
\begin{aligned}
\mathbf{1 0} & =\underbrace{(\mathbf{3}, 2)_{1 / 6}}_{q}+\underbrace{(\overline{3}, \mathbb{1})_{-2 / 3}}_{u^{c}}+\underbrace{(\mathbb{1}, \mathbb{1})_{1}}_{e^{c}}, \\
\overline{\mathbf{5}} & =\underbrace{(\overline{\mathbf{3}}, \mathbb{1})_{1 / 3}}_{d^{c}}+\underbrace{(\mathbb{1}, 2)_{-1 / 2}}_{l},
\end{aligned}
$$

where the decomposition is given with respect to the standard model gauge group $\mathrm{SU}(3) \times$ $\mathrm{SU}(2) \times \mathrm{U}(1)_{Y}$. Note that the right-handed neutrino is not automatically included and has to be added as an extra singlet. This is in contrast to the GUT group $\mathrm{SO}(10)$ [25], where a single 16 contains the above matter as well as the right-handed neutrino, $\mathbf{1 6}=\mathbf{1 0}+\overline{\mathbf{5}}+\mathbb{1}$.

In addition to the matter fields two Higgs multiplets are required for the generation of masses for the standard model particles,

$$
\begin{aligned}
& H_{u}=\mathbf{5}=\underbrace{(\mathbf{3}, \mathbb{1})}_{\text {exotic }}+\underbrace{(\mathbb{1}, \mathbf{2})_{1 / 2}}_{h_{u}}, \\
& H_{d}=\overline{\mathbf{5}}=\underbrace{(\overline{\mathbf{3}}, \mathbb{1})}_{\text {exotic }}+\underbrace{(\mathbb{1}, \mathbf{2})_{-1 / 2}}_{h_{d}},
\end{aligned}
$$

and the fact that the exotic triplet and anti-triplet have to be decoupled from the lowenergy theory is the doublet-triplet splitting problem. In orbifold models it is solved by the fact that the exotics have odd boundary conditions, while the doublets correspond to zero modes in four dimensions.

In the language of $\mathcal{N}=1$ supersymmetry, interactions are then expressed by the superpotential

$$
\begin{aligned}
W= & \mu H_{u} H_{d}+\mu_{i} H_{u} \overline{\mathbf{5}}_{(i)}+C_{i j}^{(u)} \mathbf{1 0}_{(i)} \mathbf{1 0}_{(j)} H_{u}+C_{i j}^{(d)} \overline{\mathbf{5}}_{(i)} \mathbf{1 0}_{(j)} H_{d} \\
& +C_{i j k}^{(R)} \overline{\mathbf{5}}_{(i)} \mathbf{1 0} \overline{\mathbf{1}}_{(j)} \overline{\mathbf{5}}_{(k)}+C_{i j}^{(L)} \overline{\mathbf{5}}_{(i)} H_{u} \overline{\mathbf{5}}_{(k)} H_{u}+C_{i j k l}^{(B)} \mathbf{1 0}_{(i)} \mathbf{1 0}_{(j)} \mathbf{1 0}_{(k)} \overline{\mathbf{5}}_{(l)},
\end{aligned}
$$

where we included dimension-five operators but no higher non-renormalizable couplings. $\mu_{i}$ and $C^{(R)}$ yield the well known renormalizable baryon $(\mathrm{B})$ and lepton $(\mathrm{L})$ number violating interactions, and the coefficients $C^{(L)}$ and $C^{(B)}$ of the dimension-five operators are usually obtained by integrating out states with masses $\mathcal{O}\left(M_{\mathrm{GUT}}\right)$.

In supergravity theories also the expectation value of the superpotential is important since it determines the gravitino mass. One expects

$$
\langle W\rangle \sim \mu \sim M_{\mathrm{EW}}
$$

if the scale $M_{\mathrm{EW}}$ of electroweak symmetry breaking is related to supersymmetry breaking. 
Experimental bounds on the proton lifetime and lepton number violating processes imply

$$
\mu_{i} \ll \mu, \quad C^{(R)} \ll 1, \quad C^{(B)} \ll 1 / M_{\mathrm{GUT}} .
$$

Furthermore, one has to accommodate the hierarchy between the electroweak scale and the GUT scale, $M_{\mathrm{EW}} / M_{\mathrm{GUT}}=\mathcal{O}\left(10^{-14}\right)$. On the other hand, lepton number violation should not be too much suppressed, since $C^{(L)} \sim 1 / M_{\mathrm{GUT}}$ yields the right order of magnitude for neutrino masses.

Convincing realizations of the above bounds can be found from symmetry arguments. Exact symmetries completely forbid the presence of couplings, while 'mildly broken' symmetries induce 'approximate symmetries' [50] and may lead to sufficient suppression.

Dimension-four proton decay operators can be forbidden by 'matter parity' [81], which arises as an unbroken $\mathbb{Z}_{2}$ subgroup of $\mathrm{U}(1)_{B-L}$ in the standard model. This mechanism can be lifted to the SU(5) GUT description by the extension of the symmetry by the additional Abelian factor $\mathrm{U}(1)_{X}$,

$$
\mathrm{SU}(5) \times \mathrm{U}(1)_{X} \subset \mathrm{SO}(10)
$$

with charge assignments

$$
t_{X}(\mathbf{1 0})=\frac{1}{5}, \quad t_{X}(\overline{\mathbf{5}})=-\frac{3}{5}, \quad t_{X}\left(H_{u}\right)=-\frac{2}{5}, \quad t_{X}\left(H_{d}\right)=\frac{2}{5} .
$$

This symmetry has the consequence $\mu_{i}=C^{(R)}=C^{(L)}=0$. Together with the hypercharge in $\mathrm{SU}(5)$ it yields $\mathrm{U}(1)_{B-L}$,

$$
\mathrm{SU}(5) \times \mathrm{U}(1)_{X} \supset G_{\mathrm{SM}} \times \mathrm{U}(1)_{B-L}, \quad t_{B-L}=t_{X}+\frac{4}{5} t_{Y} .
$$

The wanted result, $\mu_{i}=C^{(R)}=0, C^{(L)} \neq 0$, can be obtained with a $\mathbb{Z}_{2}^{X}$ subgroup of $\mathrm{U}(1)_{X}$, which contains the matter parity $P_{X}$,

$$
P_{X}(\mathbf{1 0})=P_{X}(\overline{\mathbf{5}})=-1, \quad P_{X}\left(H_{u}\right)=P_{X}\left(H_{d}\right)=1 .
$$

Matter parity, however, does not solve the problem $C^{(B)} \neq 0$, and also the hierarchy $M_{\mathrm{EW}} / M_{\mathrm{GUT}} \ll 1$ remains unexplained.

Note that the effective model, as derived from the heterotic string, does not contain a continuous $\mathrm{U}(1)_{R}$ symmetry which forbids the $\mu$-term or dimension-five proton decay operators. Instead, three discrete $R$-symmetries are present, and in principle they can be sufficient. However, as we shall see, the phenomenology of a model strongly depends on the chosen vacuum and it is difficult to fulfill all low-energy constraints simultaneously. It is a main motivation for the present work to get a better understanding of the relation between orbifold vacua and phenomenology, and to explore new tools for the selection of candidates for physical vacua. 


\subsection{Standard model families and a unique $\mathrm{U}(1)_{B-L}$}

In the previous chapter, the spectrum of an effective orbifold GUT in six dimensions was calculated, which followed from a specific compactification of the heterotic string. The latter was an orbifold which was designed to produce the content of a $\mathbf{1 6}$ of the grand unifying group $\mathrm{SO}(10)$, localized at two fixed points [13]. The effective local GUT at the fixed points $n_{2}=0,1$ is a $\mathrm{SU}(5)$ theory, and therefore these two families take the form (cf. (192), (248))

$$
\begin{array}{ll}
\left(n_{2}, n_{2}^{\prime}\right)=(0,0): & \mathbf{1 0}_{(1)}+\overline{\mathbf{5}}_{(1)}+N_{(1)}^{c}, \\
\left(n_{2}, n_{2}^{\prime}\right)=(0,1): & \mathbf{1 0}_{(2)}+\overline{\mathbf{5}}_{(2)}+N_{(2)}^{c} .
\end{array}
$$

They arise from the first twisted sector and are localized at the origin of the compact $\mathrm{SU}(3)$-plane, described by $n_{3}=0$, cf. Figure 2 . The charges of the chiral multiplets under the six local U(1) factors at the fixed points are listed in Table 8.

The fields which combine to the third standard model family in four dimensions have to come from the bulk. This is interesting, because also the Higgs multiplets have to be bulk fields, since they should couple directly to both localized families. One can then expect that the bulk family is naturally heavier than the two localized ones, which matches with the structure of the low-energy standard model. Table 10 shows that at $n_{2}=0$, there are in fact two ten-plets with origin in the bulk, both coming from the untwisted sector,

$$
10_{(3)} \equiv 10, \quad 10_{(4)} \equiv \overline{\mathbf{1 0}}^{c} .
$$

Note that $\mathbf{1 0}$ is the left-handed chiral multiplet component $H_{L}$ of the bulk hypermultiplet $H=\left(H_{L}, H_{R}\right)$, while $\overline{\mathbf{1 0}}^{c}$ denotes the charge conjugated right-handed component $H_{R}^{c}$. This then contains a left-handed fermion and transforms as a $\mathbf{1 0}$ of SU(5). Also the Abelian charges of this field are the inverse of those given for the hypermultiplet $H$ in Table 10 .

The interesting observation now is that both of these ten-plets are split multiplets and only the sum of their zero modes combines to the expected content of a $\mathbf{1 0}$ of SU(5), given in $(248 \mathrm{a})$ :

$$
\begin{array}{ll}
\text { Zero modes of } \mathbf{1 0}_{(3)}: & (\overline{\mathbf{3}}, \mathbb{1})=u^{c}, \quad(\mathbb{1}, \mathbb{1})=e^{c}, \\
\text { Zero modes of } \mathbf{1 0}_{(4)}: & (\mathbf{3}, \mathbf{2})=q .
\end{array}
$$

This is explicitly shown in Table 30 in Appendix E. The representations are here given with respect to the non-Abelian part of the standard model gauge group, $\mathrm{SU}(3) \times \mathrm{SU}(2)$. One also observes that all $\overline{\mathbf{5}}$-plets of $\mathrm{SU}(5)$ in the projected bulk spectrum are split multiplets. Thus we conclude that the model requires not three but four families in the six-dimensional description. This avoids the mostly incorrect mass relations of standard four-dimensional SU(5) GUTs and gives room for an interesting flavor structure of the Yukawa couplings.

The identification of quark-lepton $\overline{\mathbf{5}}$-plets is more complicated. For the left-handed chiral bulk multiplets $H_{L}, H_{R}^{c}$, associated with the states from Tables 10, 12 and their 
charge conjugates, respectively, we introduce the notation

$$
\begin{array}{ll}
k=0: & \mathbf{5}, \overline{\mathbf{5}}, \\
k=2: & \mathbf{5}_{n_{3}, \gamma}, \overline{\mathbf{5}}_{n_{3}, \gamma}, \\
k=4: & \mathbf{5}_{n_{3}, \gamma}^{c}, \overline{\mathbf{5}}_{n_{3}, \gamma} .
\end{array}
$$

The local SU(5) GUT at $n_{2}=0$ provides a hypercharge generator $t_{Y}$, under which the standard model lepton $l, e^{c}$, the quarks $q, d^{c}, u^{c}$ and the Higgs doublets $H_{u}, H_{d}$ have the following charges:

$$
\begin{array}{r}
Q_{Y}(l)=-2 Q_{Y}\left(e^{c}\right)=Q_{Y}\left(H_{d}\right)=-Q_{Y}\left(H_{u}\right)=-\frac{1}{2}, \\
Q_{Y}\left(u^{c}\right)=-2 Q_{Y}\left(d^{c}\right)=-4 Q_{Y}(q)=-\frac{2}{3} .
\end{array}
$$

Inspection of the zero modes, listed in Table 30 in Appendix E, then suggests the following classification for the $\mathbf{5}, \overline{\mathbf{5}}$-plets at $n_{2}=0$, cf. (248b), (249):

Quark-like zero modes, $d^{c}=(\overline{\mathbf{3}}, \mathbb{1}): \quad \overline{\mathbf{5}}_{0, \frac{1}{2}}, \mathbf{5}_{0,0}^{c}, \mathbf{5}_{0,1}^{c}, \overline{\mathbf{5}}_{2,0}, \overline{\mathbf{5}}_{2,1}$,

Lepton- or $H_{d}$-like zero modes, $(\mathbb{1}, \mathbf{2}): \quad \overline{\mathbf{5}}_{1,0}, \overline{\mathbf{5}}_{1,1}, \mathbf{5}_{1, \frac{1}{2}}^{c}, \mathbf{5}_{2,0}^{c}, \mathbf{5}_{2,1}^{c}, \overline{\mathbf{5}}$,

$H_{u}$-like zero modes, $(\mathbb{1}, \mathbf{2})$ :

$\mathbf{5}_{1,0}, \mathbf{5}_{1,1}, \overline{\mathbf{5}}_{1, \frac{1}{2}}^{c}, \mathbf{5}_{2, \frac{1}{2}}, \mathbf{5}$

Exotic zero modes :

$$
\mathbf{5}_{0, \frac{1}{2}}, \overline{\mathbf{5}}_{0,0}^{c}, \overline{\mathbf{5}}_{0,1}^{c}, \overline{\mathbf{5}}_{2, \frac{1}{2}}^{c} \text {. }
$$

So far the spectrum still seems to be rather ambiguous. However, we can now benefit from the local SU(5) GUT structure at $n_{2}=0$. As it was described in Section 4.1, dangerous dimension-four proton decay operators can be forbidden in the $\mathrm{SU}(5)$ language by an additional Abelian factor, called $\mathrm{U}(1)_{X}$. This symmetry plays the role of $\mathrm{U}(1)_{B-L}$ and in fact only differs from it by hypercharge. Such a symmetry should also be present at $n_{2}=0$. We can make an ansatz for the generator (255),

$$
\begin{aligned}
G_{\mathrm{SM}} \times \mathrm{U}(1)_{B-L} & \subset \mathrm{SU}(5) \times \mathrm{U}(1)_{X} \subset \mathrm{SU}(6) \times \mathrm{U}(1)^{5}, \\
t_{X} & =\sum_{i=1}^{6} a_{i} t_{i}, \quad t_{6} \equiv t_{6}^{0}, \\
t_{B-L} & =t_{X}+\frac{4}{5} t_{Y},
\end{aligned}
$$

with coefficients $a_{1}, \ldots, a_{6}$. We have already identified two localized families in (257), and two 10-plets of two bulk families in (258). This fixes four of the coefficients, $a_{1}=a_{2}=$ $2 a_{4}, a_{3}=-1 / 3, a_{6}=1 / 15$, and leads to

$$
\begin{aligned}
& Q_{X}(\mathbf{5})=Q_{X}\left(\overline{\mathbf{5}}_{0,0}^{c}\right)=Q_{X}\left(\overline{\mathbf{5}}_{0,1}^{c}\right)=-\frac{2}{5}, \\
& Q_{X}(\overline{\mathbf{5}})=Q_{X}\left(\overline{\mathbf{5}}_{0, \frac{1}{2}}\right)=Q_{X}\left(\mathbf{5}_{0,0}^{c}\right)=Q_{X}\left(\mathbf{5}_{0,1}^{c}\right)=\frac{2}{5} .
\end{aligned}
$$


From the list of candidates for $d^{c}$-quarks in (264a), only $\overline{\mathbf{5}}_{2,0}$ and $\overline{\mathbf{5}}_{2,1}$ can be consistent with such a symmetry. These fields have identical charges, and the requirement that both the charge under $\mathrm{U}(1)_{X}$ and $\mathrm{U}(1)_{B-L}$ should take the physical values fixes the remaining two coefficients, $a_{1}=1, a_{5}=1 / 6$. We have thus found a unique $\mathrm{U}(1)_{X}$ at $n_{2}=0$, implying a unique $\mathrm{U}(1)_{B-L}$ in four dimensions:

$$
\begin{aligned}
\mathrm{U}(1)_{X}: & t_{X} & =t_{1}+t_{2}-\frac{1}{3} t_{3}+\frac{1}{2} t_{4}+\frac{1}{6} t_{5}+\frac{1}{15} t_{6}^{0}, \\
\mathrm{U}(1)_{B-L}: & t_{B-L} & =t_{X}+\frac{4}{5} t_{Y} .
\end{aligned}
$$

The embedding of these generators into $\mathrm{E}_{8} \times \mathrm{E}_{8}$ can be found in Table 4 .

Consistency with $\mathrm{U}(1)_{X}$ and $\mathrm{U}(1)_{B-L}$ reshuffles the list of physical and unphysical 5and $\overline{\mathbf{5}}$-plets,

Quark-like zero modes, $d^{c}=(\overline{\mathbf{3}}, \mathbb{1}): \quad \overline{\mathbf{5}}_{2,0}, \overline{\mathbf{5}}_{2,1}$,

Lepton-like zero modes, $(\mathbb{1}, \mathbf{2}): \quad \mathbf{5}_{2,0}^{c}, \mathbf{5}_{2,1}^{c}$,

$H_{d}$-like zero modes, $(\mathbb{1}, \mathbf{2})$ :

$\overline{\mathbf{5}}_{1,0}, \overline{\mathbf{5}}_{1,1}, \mathbf{5}_{1, \frac{1}{2}}^{c}, \overline{\mathbf{5}}$

$H_{u}$-like zero modes, $(\mathbb{1}, 2)$ :

$\mathbf{5}_{1,0}, \mathbf{5}_{1,1}, \overline{\mathbf{5}}_{1, \frac{1}{2}}^{c}, \mathbf{5}$

Exotic zero modes :

$$
\mathbf{5}_{0, \frac{1}{2}}, \overline{\mathbf{5}}_{0,0}^{c}, \overline{\mathbf{5}}_{0,1}^{c}, \overline{\mathbf{5}}_{2, \frac{1}{2}}^{c}, \overline{\mathbf{5}}_{0, \frac{1}{2}}, \mathbf{5}_{0,0}^{c}, \mathbf{5}_{0,1}^{c} \mathbf{5}_{2, \frac{1}{2}} \text {. }
$$

Up to the doubling due to $\gamma=0,1$, we have uniquely identified the missing matter $\overline{\mathbf{5}}$-plets of the two bulk families.

\section{$4.3 \quad F$-terms and $D$-terms}

The driving force for non-zero vacuum expectation values of fields present in the model are the conditions for unbroken supersymmetry (cf. [47]). As stated before, the false vacuum (246), which was obtained compactifying the heterotic string on an orbifold, does not maintain supersymmetry since the corresponding symmetry transformations do not close in the presence of non-vanishing Fayet-Iliopoulos D-terms. The latter correspond to auxiliary fields of the vector multiplets that are associated with the $\mathrm{U}(1)$ factors which locally have non-zero traces. The corresponding generators and the traces were calculated in Section 3.3.3.

The $\mathcal{N}=2$ vector multiplet has in fact three auxiliary fields $D_{1}, D_{2}, D_{3}$ which form a triplet under $\mathrm{SU}(2)_{R}$ and must all vanish in the bulk. However, at the fixed points half of the supersymmetry is broken and the local $\mathcal{N}=1$ vector multiplet has an effective $D$-term $D \equiv-D_{3}+F_{56}$, where $F_{56}$ is the associated field strength in the $z^{5}, z^{6}$ direction. Thus the local $D$-term cancelation condition at $n_{2}=0$ (cf. $[46,79,82]$ ),

$$
D_{3}^{a}=F_{56}^{a}=\frac{g M_{P}^{2}}{384 \pi^{2}} \frac{\operatorname{tr} t_{a}}{\left|t_{a}\right|^{2}}+\sum_{i} q_{i}^{a}\left|s_{i}\right|^{2},
$$


where $q_{i}^{a}$ is the $\mathrm{U}(1)_{a}$ charge of the singlet $s_{i}$, has always a solution, even for non-vanishing right-hand-side. This means that in principle localized FI-terms do not necessarily induce singlet vevs and the corresponding $\mathrm{U}(1)$ can remain unbroken. However, since our model has distinct anomalous $\mathrm{U}(1)$ factors at the inequivalent fixed points $n_{2}=0,1$ and a nonvanishing net anomalous $\mathrm{U}(1)$ in four dimensions, its global $D$-flat solution cannot be of that kind. We rather expect a mixture of singlet vevs and a nontrivial gauge background $\left\langle F_{56}^{\mathrm{an}}\right\rangle$.

Note that the Fayet-Iliopoulos term in (271) is of GUT scale size,

$$
\xi \sim \frac{M_{P}}{\sqrt{384 \pi^{2}}} \sim M_{\mathrm{GUT}}
$$

and appears as an explicit scale in the effective model. In fact, the abovementioned expectation value of a bulk hypermultiplet can in principle be used to transfer this scale to the effective potential of the volume modulus. After supersymmetry breaking, this contribution in combination with the Casimir energy can indeed stabilize the size of the effective orbifold at the GUT scale $[37,45]$.

Further conditions arise from the $F$-terms. Using the formulation in terms of $\mathcal{N}=1$ superfields, which was introduced in Section 3.2.7, the $F$-term conditions are ${ }^{21}$

$$
F_{\phi}=\frac{\partial W}{\partial \phi}=0, \quad \text { for all fields } \phi
$$

In the following we shall assume that the equations (271) and (273) can be solved by giving expectation values to a selection of singlets in the model. The collection of the latter is denoted by the symbol $\mathcal{S}$ and called a vacuum of the model. However, the proof that this can be done for the suggested sets will not be given in this work. Due to the large number of contributing fields this is difficult to study, especially in the six-dimensional setup. One may hope that phenomenological requirements are a good guide towards solutions, since the physical vacuum, if existent among all possibilities within the model, has to be supersymmetric $^{22}$. We will only briefly return to the explicit calculation of the $F$-terms at the end of this chapter.

\subsection{Decoupling of exotics}

The most severe phenomenological requirement at this point is the decoupling of the exotic fields at the GUT fixed points $n_{2}=0$. Namely, these are 16 5- or $\overline{\mathbf{5}}$-plets of SU(5) from the list (264) which have unwanted zero modes, additionally to the required third matter generation.

\footnotetext{
${ }^{21}$ These are $F$-terms with respect to global supersymmetry transformations. All vacua that we will study in the following have the property $\langle W\rangle=0$, such that they coincide with the $F$-terms of a supergravity description.

${ }^{22}$ Note that the scale of the Fayet-Iliopoulos term in (271) is too large for a phenomenologically interesting supersymmetry breaking scenario via the above $D$-terms.
} 


\begin{tabular}{|lll}
\hline$S_{3} S_{7} X_{2}^{c}$, & $S_{3} S_{6} Y_{0}^{* c}$, & $S_{2} S_{3} Y_{0}^{c}$ \\
$S_{1} S_{3} X_{0}^{c}$, & $S_{4} S_{5} Y_{0}^{* c}$, & $S_{1} S_{5} \bar{Y}_{0}^{c}$ \\
$S_{2} S_{5} \bar{X}_{0}^{c}$ & & \\
\hline
\end{tabular}

Table 14: Monomials to order three with the properties $\mathbf{Q}=0, \mathbf{R}=(0,0,-1,0)$, up to the order of the twist in the three compact planes.

The exotics in the model are vector-like. One thus has the possibility to decouple pairs of $\mathbf{5}$ 's and $\overline{\mathbf{5}}$ 's by generating supersymmetric mass terms for these fields,

$$
W=m \mathbf{5} \overline{\mathbf{5}}=\left\langle s_{1} \cdots s_{M}\right\rangle \mathbf{5} \overline{\mathbf{5}}, \quad s_{i}: \text { Singlets. }
$$

This requires that the fields $s_{i}$ are singlets of the gauge group that should remain unbroken in this process, which contains the local $\mathrm{SU}(5)$ in the case of interest. Furthermore, one assumes that the scalar potential has a supersymmetric minimum which corresponds to non-vanishing vacuum expectation values (vevs) for the $s_{i}$, as discussed in the previous section.

The analysis of the local decoupling at the SU(5) fixed points is much easier than its analogue in the effective four-dimensional field theory. The reason is that many exotics can be decoupled in one step, by the use of only three singlets. From Table 12 we read off that for each left-handed chiral multiplet from the bulk, there is also at least one right-handed chiral multiplet with exactly the same charges. They only differ by the superposition quantum number $\gamma$. One can thus build the complete gauge invariants

$$
\mathbf{5}_{n_{3}} \mathbf{5}_{n_{3}}^{c}, \quad \overline{\mathbf{5}}_{n_{3}} \overline{\mathbf{5}}_{n_{3}}^{c}, \quad \Rightarrow \quad \mathbf{Q}=0, \quad \mathbf{R}=(-1,-1,0,0),
$$

where we dropped the sub-label $\gamma$ and gave the $R$-charges of the bosonic components. Allowed superpotential terms have to fulfill the rule (91), $\sum \mathbf{R}=(-1,-1,-1,0)$ for bosonic components, up to the order of the twist in the three planes. One can thus generate a universal mass term for all above pairs by one monomial of singlets, of order three:

$$
M_{*}=\left\langle\bar{X}_{0}^{c} S_{2} S_{5}\right\rangle \quad \Rightarrow \quad \mathbf{Q}=0, \quad \mathbf{R}=(0,0,-1,0) .
$$

This combination is allowed by all selection rules (128). Note that this is not the only possible choice, the complete list at order three is given in Table 14. For each choice, a specific combination of the six local $\mathrm{U}(1)$ factors is broken ${ }^{23}$.

The mass (276) leads to a universal decoupling of six pairs of vector-like exotics:

$$
W \supset M_{*}\left(\mathbf{5}_{0} \mathbf{5}_{0}^{c}+\overline{\mathbf{5}}_{0} \overline{\mathbf{5}}_{0}^{c}+\mathbf{5}_{1} \mathbf{5}_{1}^{c}+\overline{\mathbf{5}}_{1} \overline{\mathbf{5}}_{1}^{c}+\mathbf{5}_{2} \mathbf{5}_{2}^{c}+\overline{\mathbf{5}}_{2} \overline{\mathbf{5}}_{2}^{c}\right) .
$$

Here we suppressed the $\gamma$ values of the decoupled fields, and from now on we shall also drop them for the remaining combinations. In Section 4.5.1 it will be shown that this is

\footnotetext{
${ }^{23}$ In [16] we chose the combination $S_{1} S_{5} \bar{Y}_{0}^{c}$. As it turned out, this does not allow the definition of a $\mathrm{U}(1)_{B-L}$ which is spontaneously broken to matter parity.
} 
justified, since they do not influence any of the relevant couplings in $W$. We are now in the position to summarize the four quark-lepton families which are present in the model:

\begin{tabular}{|c|c|c|}
\hline Family (1): & $\mathbf{1 0} \mathbf{0}_{(1)}, \overline{\mathbf{5}}_{(1)}$ & $\left(n_{2}, n_{2}^{\prime}\right)=(0,0)$, \\
\hline Family (2): & $\mathbf{1 0} \mathbf{0}_{(2)}, \overline{\mathbf{5}}_{(2)}$, & $\left(n_{2}, n_{2}^{\prime}\right)=(0,1)$, \\
\hline Family (3): & $\mathbf{1 0}_{(3)}=\mathbf{1 0}, \overline{\mathbf{5}}_{(3)}=\mathbf{5}_{2}^{c}$, & (bulk), \\
\hline Family (4): & $\mathbf{1 0} 0_{(4)}=\overline{\mathbf{1 0}}^{c}, \overline{\mathbf{5}}_{(4)}=\overline{\mathbf{5}}_{2}$, & (bulk). \\
\hline
\end{tabular}

The right-handed neutrinos $N_{(i)}^{c}$ are omitted in the discussion, due to the large number of singlets in the model. Recall from (270) or from Table 30 in Appendix E that the two bulk families give rise to only one standard model family in four dimensions. For the Higgs multiplets $H_{u}, H_{d}$ an ambiguity remains,

$$
\underbrace{\mathbf{5}, \mathbf{5}_{1}}_{H_{u} \text {-like }}, \quad \underbrace{\overline{\mathbf{5}}, \overline{\mathbf{5}}_{1}}_{H_{d} \text {-like }} .
$$

Furthermore, one is left with one single pair of vector-like exotics $\mathbf{5}_{0}^{c}, \overline{\mathbf{5}}_{0}^{c}$, which can again be decoupled with a set of three singlets,

$$
W \supset M_{*}^{\prime} \overline{\mathbf{5}}_{0}^{c} \mathbf{5}_{0}^{c}, \quad M_{*}^{\prime}=\left\langle X_{0} S_{2} S_{5}\right\rangle .
$$

In summary, the local $\mathrm{SU}(5) \times \mathrm{U}(1)_{X}$ GUT structure at the fixed points $n_{2}=0$ leads to a significant simplification of the discussion. In (268) and (269) we identified uniquely the generators of $\mathrm{U}(1)_{X}$ and $\mathrm{U}(1)_{B-L}$. After a first step of universal decoupling (277) using only three singlet fields, these symmetries fixed the definition of four standard model families in the $\mathrm{SU}(5)$ language, as given in (278). One remaining pair of exotics could again be decoupled by a singlet monomial of order three, extending the list of singlets needed for the decoupling to

$$
X_{0}, \bar{X}_{0}^{c}, S_{2}, S_{5}
$$

So far unsolved is the question which combination of fields from (279) plays the role of the two Higgs multiplets $H_{u}, H_{d}$. The remaining multiplets will then constitute another pair of exotics, and consequently require the generation of another mass term.

\subsubsection{A minimal vacuum}

The transition from the false vacuum, $\left\langle s_{i}\right\rangle=0$ for all $s_{i}$, to the physical vacuum $\mathcal{S}$,

$$
\mathcal{S}=\{s \mid\langle s\rangle \neq 0\}
$$

is required for three reasons: Supersymmetry has to be maintained in the perturbative vacuum solution, all exotics have to be decoupled, and the additional gauge symmetry has to be broken, under which the standard model fields transform in the effective theory. 
The latter point states that a Higgs mechanism is needed in order to make the gauge fields heavy, which are associated with the nine extra $U(1)$ factors in four dimensions, including the six at $n_{2}=0$. This implies the spontaneous breaking of $\mathrm{U}(1)_{X}$ and $\mathrm{U}(1)_{B-L}$. However, the dimension-four proton decay operators only remain absent if a global matter parity survives the breaking. In the present model, this can be achieved by assuming vevs of singlets with charges \pm 2 under $\mathrm{U}(1)_{X}$. This leads to the breaking of the latter factor to a parity (or a group of even order), denoted as $P_{X}$. From Table 32 in Appendix $\mathrm{F}$ one finds that there are only two such singlet fields in the model, $U_{2}$ and $U_{4}$ from the untwisted sector,

$$
\left\langle U_{2}\right\rangle \neq 0 \text { or }\left\langle U_{4}\right\rangle \neq 0: \quad \mathrm{U}(1)_{X} \rightarrow \mathbb{Z}_{M}^{X} \supset P_{X}
$$

for some even integer $M$. Note that these fields do not have zero modes in four dimensions, but are present as bulk fields of the six-dimensional theory, which have positive parity at the GUT fixed points $n_{2}=0$. Their corresponding mass term is then expected to be of the size of the compactification scale. However, if this is of the order of the GUT scale it coincides with the size of the Fayet-Iliopoulos scale, and vevs of the fields can contribute to vacua.

With the above requirement, we can formulate a minimal vacuum $\mathcal{S}_{0}$ which leads to the desired decoupling of exotics $(277),(280)$, and breaks $\mathrm{U}(1)_{X}$ to the parity $P_{X}$, which yields matter parity in four dimensions. We shall thus demand that the vacuum

$$
\mathcal{S}_{0}=\left\{X_{0}, \bar{X}_{0}^{c}, U_{2}, U_{4}, S_{2}, S_{5}\right\}
$$

is contained in all sets of singlets which are studied as candidates for physical vacua in the following ${ }^{24}$.

\subsection{Unbroken symmetries}

The phenomenology of a string model is determined by the spectrum and the interactions of the low-energy effective theory. The knowledge of the unbroken symmetries in a given vacuum $\mathcal{S}$ is essential for an understanding of the latter. In the present setup, couplings among fields of the effective theory arise from higher-dimensional operators, after the transition to the physical vacuum,

$$
W=\left\langle s_{1} \cdots s_{N}\right\rangle \phi_{1} \cdots \phi_{M}, \quad s_{i} \in \mathcal{S}, \quad \phi_{i} \notin \mathcal{S} .
$$

Here the fields $\phi_{i}$ can play the role of matter multiplets, for which the effective Lagrangian is desired. Furthermore, the mass terms (277), (280) are examples of couplings which have to be generated for a viable phenomenology.

In the following we describe how the unbroken symmetries in a physical vacuum can be determined, and study the minimal vacuum $\mathcal{S}_{0}$ from (284) as an example. The methods

\footnotetext{
${ }^{24}$ It is not minimal in the strict sense: Only the presence of one of the fields $U_{2}, U_{4}$ is required to fulfill the constraints. However, we will not study complete scans over all possibilities in the following, but only focus on some interesting examples.
} 
introduced here enable us to identify it as a vacuum which does not suffer from dimensionfour proton decay operators ${ }^{25}$, but contains two light Higgs pairs in the spectrum.

\subsubsection{Symmetries of the false vacuum}

As discussed in Sections 2.4 and 3.1.2, the terms which appear in the superpotential are subject to a set of constraints, the string selection rules. They arise from string theory calculations, but can be interpreted in terms of symmetries of the effective field theory.

In the present model, the rules give rise to an effective symmetry

$$
G=G_{\text {gauge }} \times G_{\text {discrete }}
$$

where, at $n_{2}=0$, the gauge part is

$$
G_{\text {gauge }}=\mathrm{SU}(5) \times \mathrm{U}(1)^{4} \times\left[\mathrm{SU}(3) \times \mathrm{SO}(8) \times \mathrm{U}(1)^{2}\right],
$$

and the discrete symmetry can be identified as (cf. (91), (96), (128))

$$
G_{\text {discrete }}=\left[\tilde{\mathbb{Z}}_{6}^{R^{1}} \times \tilde{\mathbb{Z}}_{3}^{R^{2}} \times \tilde{\mathbb{Z}}_{2}^{R^{3}} \times \mathbb{Z}_{6}^{k}\right]_{R} \times \mathbb{Z}_{3}^{n_{3} k} \times \mathbb{Z}_{2}^{n_{2} k} \times \mathbb{Z}_{2}^{n_{2}^{\prime} k} .
$$

Here we use the notation that the group $\mathbb{Z}_{M}^{q}$ acts on a state with charge $q$ as

$$
\mathbb{Z}_{M}^{q}: \quad|q\rangle \mapsto g_{M}^{a}(q)|q\rangle, \quad g_{M}^{a}(q)=e^{2 \pi i \frac{a q}{M}}, \quad a=0, \ldots,(M-1) .
$$

The tilde in $\tilde{\mathbb{Z}}_{M}^{q}$ indicates that the $M$-fold application of the generating element does not reproduce the identity, but some other transformation which is generated by the remaining group factors. For example, the symmetries $\tilde{\mathbb{Z}}_{l_{i}}^{R^{i}}, i=1,2,3$, with elements $\tilde{g}_{l_{i}}^{a}\left(R^{i}\right)$, give for the choice $a=0 \bmod l_{i}$ an element of $\mathbb{Z}_{6}^{k}$. This follows from the observation

$$
R^{i}=-\frac{k}{l_{i}} \bmod 1
$$

and allows us to identify

$$
\tilde{g}_{l_{i}}^{l_{i}}\left(R^{i}\right) \equiv g_{6}^{-6 / l_{i}}(k)=g_{l_{i}}^{-1}(k) \quad l_{i}=6,3,2, \quad i=1,2,3 .
$$

This is a consequence of the statement that the selection rule $\sum k=0 \bmod 6$ can be understood as part of the discrete $R$-symmetries, cf. the discussion before (96).

The last three factors of (288) resemble the order of the sub-lattices in the planes $\mathrm{SU}(3)$ and $\mathrm{SO}(4)=\mathrm{SU}(2) \times \mathrm{SU}(2)$, respectively. A peculiarity arises from the $\mathrm{G}_{2}$-plane. There, the twists $k=2$ and $k=3$ correspond to two different sub-lattices $\Lambda_{k}$, whose sum is equivalent to the sub-lattice of full $\mathbb{Z}_{6}$ rotations, $\Lambda_{1}$. This leads to an additional symmetry

\footnotetext{
${ }^{25}$ The absence of dimension-five proton decay operators in the vacuum $\mathcal{S}_{0}$ is shown in Section 4.6.
} 
factor, which only applies to couplings with the property that all its contributing fields come from equivalent twisted sectors,

$$
G_{\text {discrete }}^{G_{2}}= \begin{cases}\mathbb{Z}_{6}^{n_{6} k}, & \text { if all fields have the same } k, \text { modulo } 6 \text { and up to a sign }, \\ \mathbb{1}, & \text { otherwise. }\end{cases}
$$

The additional restriction does not apply to all couplings in the superpotential. Therefore, it cannot be interpreted as an additional symmetry of the full system. However, physical interactions have to fulfill this constraint, and thus we are forced to write the total superpotential $W_{\text {tot }}$ as the sum of two terms,

$$
\begin{array}{ll}
W_{\text {tot }}=W_{0}+W, \quad & W_{0}: \text { Symmetry } G_{\text {gauge }} \times G_{\text {discrete }} \times \mathbb{Z}_{6}^{n_{6} k}, \\
W: \text { Symmetry } G_{\text {gauge }} \times G_{\text {discrete }}
\end{array}
$$

All couplings which only involve fields with equivalent twists, in the sense of (292), are collected in $W_{0}$. The rest, which is the majority of the couplings of interest, is contained in $W$. Note that the form (293) is the outcome of a string calculation for the effective low-energy field theory. The split structure is a direct consequence of the fact that the $\mathbb{Z}_{6-\text { II }}$ twist has two sub-twists, which both affect the torus $G_{2}$, and therefore seems to be unavoidable.

However, in the following analysis of the symmetries we shall ignore the contributions from $W_{0}$. This is justified, since order-by-order scans show that the latter play practically no role. For example, direct Yukawa-couplings among fields of one generation only arise through couplings which involve singlets from other twisted sectors. We will comment more on the terms in $W_{0}$ in Section 4.7, when we discuss the superpotential to all orders.

\subsubsection{Symmetries of physical vacua}

We now study the question which of the symmetries (286) remains unbroken after the transition form the false vacuum to the physical vacuum,

$$
G_{\text {tot }} \rightarrow G_{\text {vac }}(\mathcal{S}), \quad \mathcal{S}=\{s \mid\langle s\rangle \neq 0\} .
$$

For simplicity, we shall assume that the non-Abelian gauge symmetry in $G_{\text {gauge }}$ is not broken during this process, and focus on the Abelian factors and the discrete symmetries (288) in the following.

Consider the transformation of a singlet $s_{i} \in \mathcal{S}$ under the symmetries of the false vacuum. We can collect the relevant charges of the continuous and the discrete symmetries in two vectors $\mathbf{Q}$ and $\mathcal{K}$, respectively,

$$
\mathbf{Q}=\left(Q_{1}, \ldots, Q_{6}\right), \quad \mathcal{K}=\left(R^{1}, R^{2}, R^{3}, k, n_{3} k, n_{2} k, n_{2}^{\prime} k\right),
$$

where $Q_{u}$ denotes the charge with respect to the $\mathrm{U}(1)$ which is generated by $t_{u}$. The transformation of $s_{i}$ can then be written as

$$
s_{i} \rightarrow e^{2 \pi i(\alpha \cdot \mathbf{Q}+\mathbf{r} \cdot \mathcal{K})} s_{i}
$$




\begin{tabular}{c|c|c} 
Symmetry & $\alpha^{\prime}$ & $\mathbf{r}^{\prime}$ \\
\hline \hline$\left[\tilde{\mathbb{Z}}_{24}\right]_{R}$ & $\left(-\frac{1}{6}, 0,-\frac{1}{12},-\frac{1}{24}, 0, \frac{1}{30}\right)$ & $\left(\frac{1}{6}, 0,0,0,0,0,0\right)$ \\
{$\left[\mathbb{Z}_{6}\right]_{R}$} & $\left(\frac{5}{6},-\frac{5}{6}, 0,0,0, \frac{1}{6}\right)$ & $\left(0, \frac{5}{3}, 0,0,0,0,0\right)$ \\
\hline $\mathbb{Z}_{120}$ & $\left(\frac{1}{2}, \frac{1}{2},-\frac{1}{6}, \frac{1}{4}, 0, \frac{1}{30}\right)$ & $(0,0,0,0,0,0,0)$
\end{tabular}

Table 15: The generators of the discrete symmetries in the vacuum $\mathcal{S}_{0}$. The notation $\tilde{\mathbb{Z}}_{M}$ stands for a discrete rotation of order $M$, up to elements of the other symmetry factors. This could alternatively be expressed as $\mathbb{Z}_{A} / \mathbb{Z}_{B}$, where $A / B=M$ and $\mathbb{Z}_{B}$ is a subgroup of the remaining discrete symmetry.

with two parameter vectors

$$
\alpha=\left(\alpha_{1}, \ldots, \alpha_{6}\right), \quad \alpha_{u} \in \mathbb{R}, \quad \mathbf{r}=\left(\frac{r_{1}}{6}, \frac{r_{2}}{3}, \frac{r_{3}}{2}, \frac{r_{4}}{6}, \frac{r_{5}}{3}, \frac{r_{6}}{2}, \frac{r_{7}}{2}\right), \quad r_{i} \in \mathbb{Z} .
$$

Note that this way of writing the transformation ignores the identifications (291) and thus double-counts the common factors of the $R$-symmetries and $\mathbb{Z}_{6}^{k}$ for $r_{i} \geqslant l_{i}, i=1,2,3$.

The symmetry group of the vacuum $\mathcal{S}$ is then given by the set of transformations which act as the identity on each of the individual vevs $\left\langle s_{i}\right\rangle$,

$$
G_{\mathrm{vac}}(\mathcal{S}): \quad\left\langle s_{i}\right\rangle=e^{2 \pi i\left(\alpha^{\prime} \cdot \mathbf{Q}+\mathbf{r}^{\prime} \cdot \mathcal{K}\right)}\left\langle s_{i}\right\rangle, \quad \text { for all } s_{i} \in \mathcal{S} .
$$

Here we characterized the unbroken subgroup of the symmetries of the false vacuum by $\alpha^{\prime}, \mathbf{r}^{\prime}$.

\section{Example: The minimal vacuum $\mathcal{S}_{0}$}

As an example, consider the vacuum $\mathcal{S}_{0}$, defined in (284). The physical vacuum is an extension of this set and its symmetry will be given by a subgroup of $G_{\mathrm{vac}}\left(\mathcal{S}_{0}\right)$.

The generating elements of the unbroken symmetry directly follow from (298). From Tables 8, 10 and 12 one reads off that none of the singlets in $\mathcal{S}_{0}$ is charged under the $\mathrm{U}(1)_{5}$ with generator $t_{5}$, thus this will remain a continuous symmetry of the new vacuum. However, all other Abelian factors are broken to discrete subgroups. For the total symmetry, acting on all fields present at $\left(n_{2}, n_{2}^{\prime}\right)=(0,0)$, we find

$$
G_{\text {vac }}\left(\mathcal{S}_{0}\right)=\left[\tilde{\mathbb{Z}}_{24} \times \mathbb{Z}_{6}\right]_{R} \times \mathrm{U}(1)_{5} \times \mathbb{Z}_{120}
$$

The enlargement of the orders of the appearing $R$-symmetries is due to the mixing of the latter with generators of the six $\mathrm{U}(1)$ factors. The list of generators of $G_{\mathrm{vac}}\left(\mathcal{S}_{0}\right)$ is given in Table 15. It shows the complicated embedding of the present discrete symmetries into the symmetries of the false vacuum. The tables in Appendix F explicitly confirm that the given symmetry is unbroken in the vacuum, furthermore they list the corresponding transformation phases for all fields present at $\left(n_{2}, n_{2}^{\prime}\right)=(0,0)$.

Note the appearance of the large factor $\mathbb{Z}_{120}$. Up to the entry corresponding to the unbroken $\mathrm{U}(1)_{5}$, its generator is proportional to the generator $t_{X}$ of $\mathrm{U}(1)_{X}$, defined in $(268)$. 
We will see that this factor is indeed responsible for the absence of dimension-four proton decay operators, as argued in (283). In fact, allowed couplings should be invariant under all possible symmetry transformations, including combinations of the discrete generators and arbitrary choices for the continuous parameter $\alpha_{5}$, for example $\alpha_{5}=1 / 12$. Together with the generator of the $\mathbb{Z}_{120}$, the latter choice adds to $\frac{1}{2} t_{X}$, associated with a $\mathbb{Z}_{60}$. The naive guess for the matter parity generator would then be $15 t_{X}$. However, as it turns out this $\mathbb{Z}_{2}$ acts trivially on all singlets of the hidden $\mathrm{SO}(8)$ gauge group, including the standard model families. The symmetry which corresponds to matter parity for the singlets of the hidden sector is in fact a $\mathbb{Z}_{4}$ symmetry with respect to the full spectrum,

$$
\text { Matter parity : } \quad P_{X}=e^{2 \pi i\left(\frac{15}{2} t_{X}\right)} \text {. }
$$

This definition yields the correct parity assignments for the matter generations (278) and the Higgs candidates (279), as shown in Table 31 in Appendix F. Together with the other unbroken symmetries in (299), it determines whether a coupling may be allowed in the effective theory in the vacuum $\mathcal{S}_{0}$, or not. As will become apparent, large unbroken symmetries can be very restrictive and forbid phenomenologically favored interactions.

\subsubsection{Symmetries and interactions}

The origin of the symmetries that we study in this chapter are the string selection rules (91), (128) for couplings in the superpotential $W$. Expressed in terms of the $R$-charges of the bosonic components of chiral multiplets, a coupling which consists of two fermions and bosons otherwise, has to fulfill

$$
R^{i}(W)=-1 \bmod l_{i}, \quad l_{i}=6,3,2, \quad i=1,2,3 .
$$

In the notation of (295), the full set of constraints can be expressed as

$$
\begin{gathered}
e^{2 \pi i\left(\alpha^{\prime} \cdot \mathbf{Q}(W)+\mathbf{r}^{\prime} \cdot \mathcal{K}(W)\right)}=e^{2 \pi i \mathbf{r}^{\prime} \cdot \mathcal{K}_{\mathrm{vac}}}, \\
\mathcal{K}_{\mathrm{vac}}=(-1 \bmod 6,-1 \bmod 3,-1 \bmod 2,0 \bmod 6,0 \bmod 3,0 \bmod 2,0 \bmod 2),
\end{gathered}
$$

for all possible choices of the parameters $\alpha^{\prime}$ and $\mathbf{r}^{\prime}$ which describe the symmetry of the vacuum. Once the explicit form of these parameter sets is known, the above formulation of the selection rules allows for a fast and easy check if a given coupling is present in the superpotential, or forbidden by an exact symmetry of the vacuum. In the latter case it will be absent to all orders in the involved singlet fields.

\section{Example: The minimal vacuum $\mathcal{S}_{0}$}

We illustrate this with the simple six-field vacuum $\mathcal{S}_{0}$ from $(284)$, whose symmetries were discussed in the previous section. Consider the $\mu$-terms of the Higgs candidates from (279), which now is a $2 \times 2$ matrix,

$$
W=\mu_{i j} H_{u}^{i} H_{d}^{j}, \quad\left(H_{u}^{i}\right)=\left(\mathbf{5}, \mathbf{5}_{1}\right), \quad\left(H_{d}^{i}\right)=\left(\overline{\mathbf{5}}, \overline{\mathbf{5}}_{1}\right),
$$


where each entry of $\mu_{i j}$ consists of expectation values of monomials of singlets,

$$
\mu_{i j}=\left\langle s_{i j}^{(1)} \cdots s_{i j}^{\left(N_{i j}\right)}\right\rangle, \quad s_{i j}^{(k)} \in \mathcal{S}_{0}, \quad i, j=1,2 .
$$

The generators of the unbroken symmetries $\alpha^{\prime}, \mathbf{r}^{\prime}$ can be found in Table 15. Be definition of the latter, $\mu_{i j}$ is invariant under all symmetry transformations. The condition (302) thus only depends on the transformation of the Higgs candidates $H_{u}^{i}, H_{d}^{i}$. For the generator of the $R$-symmetry $\left[\tilde{\mathbb{Z}}_{24}\right]_{R}$,

$$
\alpha^{\prime}=\left(-\frac{1}{6}, 0,-\frac{1}{12},-\frac{1}{24}, 0, \frac{1}{30}\right), \quad \mathbf{r}^{\prime}=\left(\frac{1}{6}, 0,0,0,0,0,0\right)
$$

the condition (302) reads

$$
\underbrace{\alpha^{\prime} \cdot \mathbf{Q}\left(H_{u}^{i} H_{d}^{j}\right)+\frac{1}{6} \cdot R^{1}\left(H_{u}^{i} H_{d}^{j}\right)}_{\frac{1}{24}\left(\begin{array}{ll}
0 & 17 \\
5 & 11
\end{array}\right)}=-\frac{1}{6} \bmod 1 .
$$

From this we conclude that all $\mu$-terms are forbidden by a discrete $R$-symmetry,

$$
\left[\tilde{\mathbb{Z}}_{24}\right]_{R}: \quad \mu_{i j}=0
$$

This states that all four Higgs candidate fields are massless in the vacuum $\mathcal{S}_{0}$. This is a two-Higgs model, which may be phenomenologically acceptable, but is inconsistent with gauge coupling unification.

Similarly, one can study the dimension-four and dimension-five proton decay operators

$$
W=C_{i j k}^{(R)} \overline{\mathbf{5}}_{(i)} \mathbf{1 0} \mathbf{0}_{(j)} \overline{\mathbf{5}}_{(k)}+C_{i j k l}^{(B)} \mathbf{1 0}_{(i)} \mathbf{1 0}(j) \mathbf{1 0}_{(k)} \overline{\mathbf{5}}_{(l)},
$$

where $(i)$ labels the four families, defined in (278). As expected, the generator of the $\mathbb{Z}_{120}$, which is related to $\mathrm{U}(1)_{X}$, forbids the presence of the dimension-four term,

$$
\mathbb{Z}_{120}: \quad C_{i j k}^{(R)}=0 .
$$

The dimension-five operators are also forbidden in the present vacuum, $C_{i j k l}^{(B)}=0$, but this does not fully follow from the unbroken symmetries. This is discussed in Appendix $\mathrm{F}$ and in the following section.

Furthermore, we can study Yukawa interactions, which now depend on the choice of Higgs fields $H_{u}, H_{d}$,

$$
W=C_{i j}^{(u)} \mathbf{1 0}_{(i)} \mathbf{1 0}_{(j)} H_{u}+C_{i j}^{(d)} \overline{\mathbf{5}}_{(i)} \mathbf{1 0}_{(j)} H_{d}
$$


The appearing matrices are related to the Yukawa couplings of the three standard model families of the effective four-dimensional theory,

$$
W_{\text {Yuk }}=Y_{i j}^{(u)} u_{i}^{c} q_{j} H_{u}+Y_{i j}^{(d)} d_{i}^{c} q_{j} H_{d}+Y_{i j}^{(l)} l_{i} e_{j}^{c} H_{d},
$$

by

$$
\begin{aligned}
Y^{(u)} & =\left(\begin{array}{lll}
C_{11}^{(u)} & C_{12}^{(u)} & C_{14}^{(u)} \\
C_{21}^{(u)} & C_{22}^{(u)} & C_{24}^{(u)} \\
C_{31}^{(u)} & C_{32}^{(u)} & C_{34}^{(u)}
\end{array}\right), \\
Y^{(d)} & =\left(\begin{array}{lll}
C_{11}^{(d)} & C_{12}^{(d)} & C_{14}^{(d)} \\
C_{21}^{(d)} & C_{22}^{(d)} & C_{24}^{(d)} \\
C_{41}^{(d)} & C_{42}^{(d)} & C_{44}^{(d)}
\end{array}\right), \quad Y^{(l)}=\left(\begin{array}{ccc}
C_{11}^{(d)} & C_{12}^{(d)} & C_{13}^{(d)} \\
C_{21}^{(d)} & C_{22}^{(d)} & C_{23}^{(d)} \\
C_{31}^{(d)} & C_{32}^{(d)} & C_{33}^{(d)}
\end{array}\right) .
\end{aligned}
$$

In the vacuum $\mathcal{S}_{0}$, the couplings are forbidden for the choice $H_{u}=\mathbf{5}_{1}$ and $H_{d}=\overline{\mathbf{5}}$,

$$
\mathrm{U}(1)_{5}: \quad C_{H_{u}=\mathbf{5}_{1}}^{(u)}=0, \quad\left[\tilde{\mathbb{Z}}_{24}\right]_{R}: \quad C_{H_{d}=\overline{\mathbf{5}}}^{(d)}=0 .
$$

On the other hand, for $H_{u}=\mathbf{5}$ and $H_{d}=\overline{\mathbf{5}}_{1}$ they are (partly) allowed,

$$
\begin{gathered}
C_{H_{u}=\mathbf{5}}^{(u)}=\left(\begin{array}{cccc}
S_{2} S_{5}\left(\bar{X}_{0}^{c}\right)^{2} & S_{2}^{\prime} S_{5}\left(\bar{X}_{0}^{c}\right)^{2} & \left(S_{2}\right)^{2} S_{5}\left(\bar{X}_{0}^{c}\right)^{2} & S_{2}\left(S_{5}\right)^{2}\left(\bar{X}_{0}^{c}\right)^{2} \\
S_{2}^{\prime} S_{5}\left(\bar{X}_{0}^{c}\right)^{2} & S_{2}^{\prime} S_{5}^{\prime}\left(\bar{X}_{0}^{c}\right)^{2} & \left(S_{2}\right)^{2} S_{5}^{\prime}\left(\bar{X}_{0}^{c}\right)^{2} & S_{2}^{\prime}\left(S_{5}\right)^{2}\left(\bar{X}_{0}^{c}\right)^{2} \\
\left(S_{2}\right)^{2} S_{5}\left(\bar{X}_{0}^{c}\right)^{2} & \left(S_{2}\right)^{2} S_{5}^{\prime}\left(\bar{X}_{0}^{c}\right)^{2} & \left(S_{2}\right)^{3} S_{5}\left(\bar{X}_{0}^{c}\right)^{2} & g \\
S_{2}\left(S_{5}\right)^{2}\left(\bar{X}_{0}^{c}\right)^{2} & S_{2}^{\prime}\left(S_{5}\right)^{2}\left(\bar{X}_{0}^{c}\right)^{2} & g & S_{2}\left(S_{5}\right)^{3}\left(\bar{X}_{0}^{c}\right)^{2}
\end{array}\right) \\
C_{H_{d}=\overline{\mathbf{5}}_{1}}^{(d)}=\left(\begin{array}{cccc}
0 & 0 & 0 & 0 \\
0 & 0 & 0 & 0 \\
0 & 0 & 0 & 0 \\
S_{5} & S_{5}^{\prime} & S_{2} S_{5} & \left(S_{5}\right)^{2}
\end{array}\right),
\end{gathered}
$$

where primes denote localization at $n_{2}^{\prime}=1$ and the matrices contain examples for lowest order monomials. Note that all zeros are present up to infinite order in the singlets, since they are protected by symmetries, cf. Appendix F. The up-type Yukawa coupling between the two bulk families is proportional to the gauge coupling. This is an important consequence of the choice $H_{u}=\mathbf{5}$, due to the origin of that multiplet in the six-dimensional gauge vector. The unification of gauge interactions and Yukawa couplings in six dimensions will imply a large mass for the top quark of the four-dimensional effective theory, and is thus phenomenologically desired. However, the down-type Yukawa couplings, which are also responsible for the lepton masses in four dimensions, are obviously not satisfactory. It seems that the generation of the latter with acceptable entries also for the light families, and the simultaneous vanishing of the $\mu$-term is difficult to realize. This appears to be a generic problem of $\mathbb{Z}_{6-\text { II }}$ orbifold models. If the symmetries which protect the zeros in $Y^{(d)}$ are completely broken, we expect that also the $\mu$-term will be generated. 
The lessons that we learn from the vacuum $\mathcal{S}_{0}$ are twofold. First, new $R$-symmetries with a complicated embedding can be responsible for vanishing $\mu$-terms and dimensionfive proton decay operators. Concerning the Yukawa couplings, the Higgs choice $H_{u}=\mathbf{5}$ (and $H_{d}=\overline{\mathbf{5}}_{1}$ ) seems to be preferred, where 5 is part of the gauge vector multiplet in six dimensions.

Second, more generally, one finds that the restriction to a small set of singlets implies a large unbroken symmetry, which forbids many of the interactions. This can have positive effects for the phenomenology, like stability of the proton, but also negative, like vanishing down-type Yukawa couplings. We expect that adding more singlets to the list breaks more symmetry and thus allows the generation of more terms in the superpotential. However, also dangerous or disfavored terms, like the $\mu$-term, may then be generated at a high scale. The art of orbifold model building is thus to find the best compromise between desired and problematic terms, or in other words, too much or too little unbroken symmetry. In the following, we will study this issue more and develop an algorithm which enables one to find the largest vacua for which a choice of properties of the superpotential remains intact, thereby exploring the phenomenological limits of a given orbifold compactification.

\subsection{Vacuum selection}

Pure guesswork is an unsatisfactory approach for the definition of a physical vacuum. At the GUT fixed points there are 67 non-Abelian singlets (and 72 in four dimensions), leading to an enormous number of different possibilities. On the other hand, a dynamical approach starting from the explicit scalar potential is excluded for the same reason, there are too many degrees of freedom in order to handle the full problem. Furthermore, the numerical coefficients of the various terms in the superpotential have to be calculated from string theory for the explicit approach, which is a non-trivial task.

One therefore usually assumes that there is a supersymmetric vacuum which can describe the MSSM to some accuracy. If that is the case, the list of singlets contributing to it should be constrained by phenomenological requirements:

- The low-energy spectrum contains the supersymmetric standard model, and no exotics.

- Supersymmetry is unbroken: $D_{a}=0, F_{i}=0$ for all $a$ and $i$.

- The model has non-vanishing Yukawa interactions.

- The proton is stable (or has a sufficiently long lifetime).

- The $\mu$ term vanishes (or is strongly suppressed).

- The gravitino is massless, $\langle W\rangle=0$.

The list could be enlarged by many more details, but, as we will see, the above constraints are already quite restrictive. Typically, the vacuum expectation values of singlets are of 
the order of the localized Fayet-Iliopoulos terms, which means they are comparable to $M_{\mathrm{GUT}} \sim 10^{16} \mathrm{GeV}$. For that reason it is difficult to generate a $\mu$-term of the weak scale by higher dimensional operators. Also the breaking of supersymmetry is expected to arise from some additional mechanism, which we do not study in this work. If the latter is linked to the electroweak scale, one expects the relation (251),

$$
\langle W\rangle \sim \mu \sim M_{\mathrm{EW}}
$$

after supersymmetry breaking, and $\langle W\rangle=\mu=0$ before.

An important guideline for the definition of the vacuum is the decoupling of exotics. In Section 4.4 we described how the definitions of a unique $\mathrm{U}(1)_{X}$ and a unique $\mathrm{U}(1)_{B-L}$ selected between physical multiplets and exotics, up to an additional doubling due to the orbifold breaking of the $\mathcal{N}=2$ supersymmetry in the six-dimensional bulk. The embedding of the corresponding generators into $\mathrm{E}_{8} \times \mathrm{E}_{8}$ is expected to depend on the choice of the Wilson line in the $\mathrm{SU}(3)$-plane, which is also linked to the presence of complete standard model families at the fixed points. There is also only little choice in the breaking of $\mathrm{U}(1)_{X}$ to a matter parity, since only two singlets in the model have the required even charge under this symmetry. These arguments lead to the definition of the minimal vacuum $\mathcal{S}_{0}$ in (284), which we take as a starting point for further steps.

The ambiguity in the model up to this point consists mainly in the identification of the Higgs multiplets $H_{u}$ and $H_{d}$. Here the crucial issue is the vanishing $\mu$-term in the effective superpotential. We shall now describe how a maximal vacuum can be defined, which contains the maximally possible number of singlets, such that $\mu=0$ holds up to infinite order. However, the arguments are general and can be used to forbid also any other disfavored interaction.

\subsubsection{Maximal vacua for vanishing couplings}

Consider a vacuum $\mathcal{S}$ and a superpotential term

$$
W=\lambda \Phi, \quad \lambda=s_{1}^{n_{1}} \cdots s_{N}^{n_{N}}, \quad \Phi=\phi_{1} \cdots \phi_{M}, \quad n_{i}, N, M \in \mathbb{N},
$$

where $s_{i} \in \mathcal{S}$ and $\phi_{i} \notin \mathcal{S}$. This coupling is allowed in the false vacuum by the string selection rules, provided

$$
\begin{aligned}
\text { Continuous symmetries: } & \mathbf{Q}(\lambda \Phi)=0, \\
\text { Discrete symmetries: } & \mathcal{K}(\lambda \Phi)=\mathcal{K}_{\text {vac }},
\end{aligned}
$$

where the notation from (295) and (303) was used. These two conditions can now be solved separately. Equation (319) has multiple solutions, which can be written as

$$
\lambda=\lambda_{0} \lambda_{s}, \quad\left\{\begin{aligned}
\mathbf{Q}\left(\lambda_{s} \Phi\right) & =0, \\
\mathbf{Q}\left(\lambda_{0}\right) & =0 .
\end{aligned}\right.
$$


Here $\lambda_{s}$ corresponds to a particular solution, and the infinite multiplicity is contained in the homogeneous solutions $\lambda_{0}$. The latter form the 'charge kernel'

$$
\operatorname{ker} Q(\mathcal{S})=\left\{\lambda_{0}=s_{1}^{n_{1}} \cdots s_{N}^{n_{N}} \mid s_{i} \in \mathcal{S}, n_{i} \in \mathbb{Z}, N \in \mathbb{N}, \mathbf{Q}\left(\lambda_{0}\right)=0\right\}
$$

which is spanned by a set $\mathcal{B}$ of basis monomials. Here by convention $s^{-1}$ denotes a singlet with negative charge vectors $\mathbf{Q}, \mathcal{K}$, as compared to the singlet $s$. Note that the $\operatorname{ker} Q$ is a module, not a vector space, due to the requirement of integer exponents $n_{i} \in \mathbb{Z}$. In the end, a projection onto physical monomials $\lambda=\lambda_{0} \lambda_{s}$ with integer exponents will be necessary,

$$
\lambda_{\text {phys }}=\mathcal{P}_{\mathbb{N}} \lambda \equiv \begin{cases}\lambda, & \text { if all } n_{i} \geqslant 0 \\ 0, & \text { otherwise }\end{cases}
$$

Keeping this in mind, we can also allow negative exponents for the particular solution $\lambda_{s}$ in (321). In fact, the solution of systems of linear integer equations with positive integer solutions are the subject of a branch of applied mathematics, called linear programming. The details of this are beyond the scope of this work. In the following, all results will be formulated with projectors $\mathcal{P}_{\mathbb{N}}$ and elements of the above basis $\mathcal{B}$.

Once the particular solution $\lambda_{s}$ is fixed, covariance of the superpotential under the discrete symmetries (320) selects a subset of the elements in the charge kernel,

$$
\mathcal{K}\left(\lambda_{0}\right)=\mathcal{K}_{\text {vac }}-\mathcal{K}\left(\lambda_{s} \Phi\right)
$$

This condition is easily evaluated. Since $\operatorname{ker} Q(\mathcal{S})$ only depends on the vacuum $\mathcal{S}$, and not on the interaction $\Phi$, it has to be calculated only once. Given its basis $\mathcal{B}$, one can then consider solutions of the condition (324) term by term, for each coupling $\Phi$.

In the following, we will turn the argument around and study unwanted couplings, such as the $\mu$-term. It is then possible to use (324) as a tool for the definition of maximal vacua, in which the disfavored interaction is forbidden to infinite order in the singlets. For this one starts with a small enough set of singlets, which either does not contain a particular solution, or whose charge kernel is too small to fulfill (324). These sets can then be subsequently enlarged by more singlets, until it is impossible to circumvent the fulfillment of the constraints. This will now be illustrated by two phenomenologically interesting examples.

\subsubsection{Gauge-Higgs unification}

Recall from (279) that after the first step of decoupling, the local GUT theory at the fixed points $n_{2}=0$ contained two pairs of candidate multiplets for the Higgs fields. We now consider the case of gauge-Higgs unification,

$$
H_{u}=\mathbf{5}, \quad H_{d}=\overline{\mathbf{5}}, \quad \text { exotics: } \mathbf{5}_{1}, \overline{\mathbf{5}}_{1},
$$

corresponding to a vacuum $\mathcal{S}_{1}$ that extends the minimal vacuum from $(284), \mathcal{S}_{1} \supset \mathcal{S}_{0}$. The vacuum $\mathcal{S}_{1}$ is now constructed as a maximal vacuum with respect to the constraints 


\begin{tabular}{c|c|rrr|c|c} 
Name & Monomial & $R_{1}$ & $R_{2}$ & $R_{3}$ & $k$ & $k n_{3}$ \\
\hline$\Omega_{1}$ & $\bar{X}_{0}^{c} S_{2} S_{5}$ & 0 & 0 & -1 & 6 & 0 \\
$\Omega_{2}$ & $X_{1} Y_{2} S_{2} S_{5}$ & 0 & -1 & -1 & 6 & 6 \\
$\Omega_{3}$ & $X_{0} \bar{X}_{1} S_{5} S_{7}$ & 0 & -1 & -1 & 6 & 3 \\
$\Omega_{4}$ & $X_{0} \bar{X}_{1} Y_{2} U_{2} U_{4}$ & -3 & -2 & 0 & 6 & 6
\end{tabular}

Table 16: Basis monomials of $\operatorname{ker} Q\left(\mathcal{S}_{1}\right)$ and the corresponding discrete charges. All monomials have $k n_{2}=k n_{2}^{\prime}=0$.

\begin{tabular}{c|c|c||c|c} 
Add & Mass term for $\mathbf{5} \overline{\mathbf{5}}$ & Order & Mass term for $\mathbf{5}_{1} \overline{\mathbf{5}}_{1}$ & Order \\
\hline $\bar{Y}_{2}$ & $\left(X_{0} \bar{X}_{0}^{c} \bar{X}_{1} \bar{Y}_{2}\left(S_{5}\right)^{2}\right)^{2} \Omega_{1} \Omega_{4}$ & 20 & $\left(X_{0}\right)^{2} X_{1} \bar{X}_{1}\left(Y_{2}\right)^{2}\left(\bar{Y}_{2}\right)^{2}\left(S_{5}\right)^{4} \Omega_{2} \Omega_{4}$ & 21 \\
$\bar{Y}_{2}^{c}$ & $\left(\bar{Y}_{2}^{c} S_{2} S_{7}\right)^{2} \Omega_{1} \Omega_{4}$ & 14 & $X_{0} Y_{2}\left(\bar{Y}_{2}^{c}\right)^{2}\left(S_{2}\right)^{3}\left(S_{5}\right)^{2}\left(S_{7}\right)^{3} \Omega_{2} \Omega_{4}$ & 21 \\
$U_{1}^{c}$ & $\left(X_{0} \bar{X}_{1} Y_{2} U_{1}^{c}\right) \Omega_{2}$ & 8 & $X_{0}\left(Y_{2}\right)^{2} U_{1}^{c} S_{2} S_{5}$ & 6 \\
$U_{3}$ & $\left(\bar{X}_{0}^{c} U_{3}\left(S_{5}\right)^{2}\right)^{2} \Omega_{2} \Omega_{4}$ & 17 & $X_{0} \bar{X}_{0}^{c}\left(Y_{2}\right)^{2} U_{2}\left(U_{3}\right)^{2} U_{4}\left(S_{5}\right)^{4} \Omega_{1}$ & 15 \\
$S_{6}$ & $\left(X_{1} Y_{2} S_{2} S_{6}\right) \Omega_{4}$ & 9 & $X_{0}\left(Y_{2}\right)^{2} U_{2} U_{4} S_{2} S_{6}$ & 7
\end{tabular}

Table 17: Addition of any further field to $\mathcal{S}_{1}$ generates monomials which induce mass terms for $\mathbf{5} \overline{\mathbf{5}}$ and $\mathbf{5}_{1} \overline{\mathbf{5}}_{1}$. Shown are lowest order examples. The monomials $\Omega_{i}$ are defined in Table 16. Singlets which complete pairs of the form $A^{c} A$ are not listed, since they always allow for mass terms proportional to $\Omega_{1} A^{c} A$. We do only consider singlets which conserve matter parity.

- Matter parity $P_{X}$ is unbroken,

- The $\mu$-term vanishes to all orders,

since we want to keep the feature that the dimension-four proton decay operators remain forbidden. The $\mu$-term can be written as

$$
W=\mu \Phi, \quad \Phi=H_{u} H_{d}, \quad \mathbf{Q}(\Phi)=0, \quad \mathcal{K}(\Phi)=0,
$$

leading to $\lambda_{s}=1$, and

$$
\mu=\lambda_{0} \in \operatorname{ker} Q, \quad \mathcal{K}\left(\lambda_{0}\right)=\mathcal{K}_{\text {vac }}
$$

From (277) one infers that the combination $\Omega_{1}=\bar{X}_{0}^{c} S_{2} S_{5}$ is a basis monomial of the charge kernel of the minimal vacuum $\mathcal{S}_{0}$. In fact, the addition of $X_{0}, U_{2}, U_{4}$ does not give rise to new uncharged combinations, and the dimension of $\operatorname{ker} Q\left(\mathcal{S}_{0}\right)$ is one. Since

$$
\mathcal{K}\left(\Omega_{1}\right)=(0,0,-1,0,0,0,0) \neq \mathcal{K}_{\text {vac }}
$$


the condition (327) is violated and the $\mu$-term is absent in the vacuum $\mathcal{S}_{0}$, in agreement with (308). As a second example, consider the dimension-five proton decay operator

$$
\Phi=\mathbf{1 0}_{(1)} \mathbf{1 0}_{(1)} \mathbf{1 0}_{(1)} \overline{\mathbf{5}}_{(1)}, \quad \Rightarrow \quad \lambda_{s}=\left(X_{0}\right)^{-1} \bar{X}_{0}^{c} .
$$

Here $\lambda_{s}$ involves a negative exponent which cannot be canceled by the singlets in $\Omega_{1}$. Thus the projection (323) yields zero for any possible term, and the coupling vanishes. This cannot be explained by the unbroken symmetries, since invariance of $X_{0}$ automatically implies invariance of $X_{0}^{-1}$.

We now aim to extend the vacuum $\mathcal{S}_{0}$, without generating a $\mu$-term or breaking matter parity. The latter requires to consider only such singlets for the enlargement which are uncharged under $\mathrm{U}(1)_{X}$, or have even charges. Table 32 from Appendix F shows that these are the singlets

$$
Q_{X}=0: \quad U_{1}^{c}, U_{3}, S_{6}, S_{7}, X_{0}^{c}, \bar{X}_{0}, X_{1}, X_{1}^{c}, \bar{X}_{1}, \bar{X}_{1}^{c}, Y_{2}, Y_{2}^{c}, \bar{Y}_{2}, \bar{Y}_{2}^{c} .
$$

We find that a maximal vacuum is given by

$$
\mathcal{S}_{1}=\mathcal{S}_{0} \cup\left\{X_{1}, \bar{X}_{1}, Y_{2}, S_{7}\right\},
$$

with a charge kernel of dimension four. The corresponding basis monomials are listed in table 16. Note that from the basis monomials it is clear that the condition $R^{1}=-1 \bmod 6$ is violated for all monomials in $\operatorname{ker} Q\left(\mathcal{S}_{1}\right)$. Table 17 shows that further addition of singlets indeed generates mass terms for the Higgs fields. On the other hand, we checked to order 500 in the singlets that the $\mu$-term is absent in the vacuum $\mathcal{S}_{1}$.

For the case of gauge-Higgs unification, any monomial which appears as a contribution to $\mu$ also contributes to $\langle W\rangle[49]$, whence

$$
\langle W\rangle=\mu=0 \quad \text { to all orders. }
$$

This follows from the observation (326), which states that $H_{u} H_{d}$ is invariant under all symmetry transformations.

We can furthermore study the possibilities to generate a mass term for the pair of exotics $\mathbf{5}_{1}, \overline{\mathbf{5}}_{1}$,

$$
W=m \Phi, \quad \Phi=\mathbf{5}_{1} \overline{\boldsymbol{5}}_{1}, \quad m=\lambda_{s} \lambda_{0} .
$$

With the choice $\lambda_{s}=\left(X_{1} \bar{X}_{1}\right)^{-1}$ one obtains

$$
\mathbf{Q}\left(\lambda_{s} \mathbf{5}_{1} \overline{\mathbf{5}}_{1}\right)=0, \quad \mathcal{K}\left(\lambda_{s} \mathbf{5}_{1} \overline{\mathbf{5}}_{1}\right)=0, \quad \mathcal{K}\left(\lambda_{0}\right)=\mathcal{K}_{\text {vac }} .
$$

The latter condition is by definition not fulfillable by singlets from the vacuum $\mathcal{S}_{1}$. Hence the two Higgs pairs property of the vacuum $\mathcal{S}_{0}$ is also present here. We conclude that gaugeHiggs unification is impossible in the present model. The absence or presence of mass terms for $\mathbf{5}, \overline{\mathbf{5}}$ is closely linked, even when going beyond the vacuum $\mathcal{S}_{1}$, as demonstrated in Table 17. This table also shows that the generation of $\mu$-terms at high order seems possible, when starting from a maximal vacuum ${ }^{26}$.

\footnotetext{
${ }^{26}$ Any symmetry breakdown corresponds to an approximate symmetry, valid up to some finite order [50]. Here the latter may be large enough for an interesting phenomenology.
} 


\begin{tabular}{c|c|c} 
Symmetry & $\alpha^{\prime}$ & $\mathbf{r}^{\prime}$ \\
\hline \hline$\left[\mathbb{Z}_{6}\right]_{R}$ & $\left(\frac{5}{6}, \frac{5}{2}, 0, \frac{5}{6},-\frac{5}{6}, \frac{1}{6}\right)$ & $\left(\frac{5}{3}, 0,0,0,0,0,0\right)$ \\
{$\left[\tilde{\mathbb{Z}}_{2}\right]_{R}$} & $\left(\frac{1}{2},-\frac{1}{2}, 0,-\frac{1}{4}, \frac{1}{4}, 0\right)$ & $(0,0,1,0,0,0,0)$ \\
\hline $\mathbb{Z}_{60}$ & $\left(\frac{1}{2}, \frac{1}{2},-\frac{1}{6}, \frac{1}{4}, \frac{1}{12}, \frac{1}{30}\right)$ & $(0,0,0,0,0,0,0)$ \\
$\mathbb{Z}_{2}$ & $\left(\frac{75}{2},-\frac{15}{2}, 5, \frac{15}{2},-\frac{5}{2}, \frac{7}{2}\right)$ & $(0,0,0,0,0,0,0)$
\end{tabular}

Table 18: The generators of the unbroken symmetry in the vacuum $\mathcal{S}_{1}$.

\section{Unbroken symmetries and interactions}

The vacuum $\mathcal{S}_{0}$ suggested that $\mathbf{5}$ is preferred over $\boldsymbol{5}_{1}$ as the up-Higgs from the viewpoint of the Yukawa couplings, which vanish for the latter in that vacuum, cf. (315). Here we repeat the same analysis for the vacuum $\mathcal{S}_{1}$, and find ${ }^{27}$

$$
G_{\text {vac }}\left(\mathcal{S}_{1}\right)=\left[\mathbb{Z}_{6} \times \tilde{\mathbb{Z}}_{2}\right]_{R} \times \mathbb{Z}_{60} \times \mathbb{Z}_{2}
$$

with generators as in Table 18 . Note that the symmetry $\mathbb{Z}_{60}$ is generated by $\frac{1}{2} t_{X}$, where $t_{X}$ is the generator of $\mathrm{U}(1)_{X}$ from (268). The symmetry thus contains the matter parity (300), as expected.

The dimension-four proton decay operators from (309) are therefore absent. However, dimension-five operators which lead to rapid proton decay are generated, for example

$$
C_{1111}^{(B)}=\left(S_{5}\right)^{3}\left(S_{7}\right)^{3}\left(X_{0}\right)^{2} \bar{X}_{0}^{c}\left(\bar{X}_{1}\right)^{3} .
$$

This follows from the basis monomial $\Omega_{3}$ from Table 16, which was not present in the vacuum $\mathcal{S}_{0}$ and allows for the cancelation of the negative exponent in (329).

A further difference of the vacuum $\mathcal{S}_{1}$ compared to $\mathcal{S}_{0}$ arises for the Yukawa couplings. In the vacuum $\mathcal{S}_{0}$ all couplings for the choice $H_{u}=\mathbf{5}_{1}$ and $H_{d}=\overline{\mathbf{5}}$ were forbidden by symmetry, while the ones for $H_{u}=\mathbf{5}$ and some for $H_{d}=\overline{\mathbf{5}}_{1}$ were allowed and of low order, as shown in (315), (316). In the present vacuum, this statement is weakened. In terms of the four SU(5) families from (278) the couplings for the choice $H_{u}=\boldsymbol{5}_{1}$ and $H_{d}=\overline{\mathbf{5}}$ are

$$
C_{H_{u}=\mathbf{5}_{1}}^{(u)}=\left(\begin{array}{llll}
s^{9} & s^{9} & s^{10} & s^{10} \\
s^{9} & s^{9} & s^{10} & s^{10} \\
s^{10} & s^{10} & s^{11} & s^{11} \\
s^{10} & s^{10} & s^{11} & s^{11}
\end{array}\right), \quad C_{H_{d}=\overline{\mathbf{5}}}^{(d)}=\left(\begin{array}{llll}
0 & 0 & 0 & 0 \\
0 & 0 & 0 & 0 \\
0 & 0 & 0 & 0 \\
s^{5} & s^{5} & s^{6} & s^{6}
\end{array}\right)
$$

where $s^{n}$ denotes an monomial of singlets of order $n$. The explicit expressions can be found in Table 19. The vacuum $\mathcal{S}_{1}$ thus indeed is a two Higgs pairs vacuum, and for both pairs a similar pattern occurs in the Yukawa couplings. However, assuming that each of the

\footnotetext{
${ }^{27}$ The differences to [17] are due to the inclusion of hidden sector multiplets in the analysis. We recover the generators given there, but here they are associated with larger symmetries. Furthermore, additional symmetry factors arise.
} 


\begin{tabular}{c|c|c||c|c|c}
$i$ & $j$ & $Y_{i j}^{(u)}$ & $i$ & $j$ & $Y_{i j}^{(u)}$ \\
\hline 1 & 1 & $\left(S_{5}\right)^{2}\left(S_{7}\right)^{2}\left(X_{0}\right)^{2}\left(\bar{X}_{1}\right)^{2} Y_{2}$ & 2 & 3 & $S_{2}^{\prime}\left(S_{5}^{\prime}\right)^{2}\left(S_{7}^{\prime}\right)^{2}\left(X_{0}\right)^{2}\left(\bar{X}_{1}\right)^{2} Y_{2}$ \\
1 & 2 & $S_{5} S_{5}^{\prime}\left(S_{7}\right)^{2}\left(X_{0}\right)^{2}\left(\bar{X}_{1}\right)^{2} Y_{2}$ & 2 & 4 & $\left(S_{5}^{\prime}\right)^{3}\left(S_{7}^{\prime}\right)^{2}\left(X_{0}\right)^{2}\left(\bar{X}_{1}\right)^{2} Y_{2}$ \\
1 & 3 & $S_{2}\left(S_{5}\right)^{2}\left(S_{7}\right)^{2}\left(X_{0}\right)^{2}\left(\bar{X}_{1}\right)^{2} Y_{2}$ & 3 & 3 & $\left(S_{2}\right)^{2}\left(S_{5}\right)^{2}\left(S_{7}\right)^{2}\left(X_{0}\right)^{2}\left(\bar{X}_{1}\right)^{2} Y_{2}$ \\
1 & 4 & $\left(S_{5}\right)^{3}\left(S_{7}\right)^{2}\left(X_{0}\right)^{2}\left(\bar{X}_{1}\right)^{2} Y_{2}$ & 3 & 4 & $S_{2}\left(S_{5}\right)^{3}\left(S_{7}\right)^{2}\left(X_{0}\right)^{2}\left(\bar{X}_{1}\right)^{2} Y_{2}$ \\
2 & 2 & $\left(S_{5}^{\prime}\right)^{2}\left(S_{7}^{\prime}\right)^{2}\left(X_{0}\right)^{2}\left(\bar{X}_{1}\right)^{2} Y_{2}$ & 4 & 4 & $\left(S_{5}\right)^{4}\left(S_{7}\right)^{2}\left(X_{0}\right)^{2}\left(\bar{X}_{1}\right)^{2} Y_{2}$ \\
\hline \hline$i$ & $j$ & $Y_{i j}^{(d)}$ & $i$ & $j$ & $Y_{i j}^{(d)}$ \\
\hline 4 & 1 & $S_{2}\left(S_{5}\right)^{2} \bar{X}_{0}^{c} \bar{X}_{1}$ & 4 & 3 & $\left(S_{2}\right)^{2}\left(S_{5}\right)^{2} \bar{X}_{0}^{c} \bar{X}_{1}$ \\
4 & 2 & $S_{2}^{\prime}\left(S_{5}^{\prime}\right)^{2} \bar{X}_{0}^{c} \bar{X}_{1}$ & 4 & 4 & $S_{2}\left(S_{5}\right)^{3} \bar{X}_{0}^{c} \bar{X}_{1}$
\end{tabular}

Table 19: Examples of leading order monomials for the Yukawa couplings $Y_{i j}^{(u)}=Y_{j i}^{(u)}$ and $Y_{i j}^{(d)}$ for the choice $H_{u}=\mathbf{5}_{1}$ and $H_{d}=\overline{\mathbf{5}}$ in the vacuum $\mathcal{S}_{1}$.

singlets contributes a suppression factor in the appropriate units, one observes a significant hierarchy in the coupling to matter for the two Higgs pair choices.

We do not follow the phenomenology of this vacuum any further, since we do not expect that gauge coupling unification is compatible with the two Higgs scenario.

\subsubsection{Partial gauge-Higgs unification}

The main reason for considering gauge-Higgs unification in the context of this model is the large top-quark mass which follows from identifying the up-type Yukawa coupling with the gauge interaction in the bulk. However, this result does only rely on the choice $H_{u}=\mathbf{5}$ and is independent of the down-Higgs. We are thus free to study the assignment

$$
H_{u}=\mathbf{5}, \quad H_{d}=\overline{\mathbf{5}}_{1}, \quad \text { exotics: } \mathbf{5}_{1}, \overline{\mathbf{5}},
$$

as suggested by the Yukawa matrices (315) and (316) for the vacuum $\mathcal{S}_{0}$. We call this scenario 'partial gauge-Higgs unification', since only the $\mathbf{5}$ arises from bulk gauge interactions, while $\overline{\mathbf{5}}_{1}$ is a field from the second twisted sector.

The $\mu$-term can then be written as

$$
W=\mu \Phi, \quad \Phi=\mathbf{5} \overline{5}_{1}, \quad \mu=\lambda_{0} \lambda_{s},
$$

and the choice $\lambda_{s}=\left(\bar{X}_{1}\right)^{-1}$ leads to

$$
\mathbf{Q}\left(\lambda_{s} 5 \overline{\mathbf{5}}_{1}\right)=0, \quad \mathcal{K}\left(\lambda_{s} 5 \overline{\mathbf{5}}_{1}\right)=(0,0,-1,0,0,0,0) .
$$

Up to the projection (323), the conditions for the monomials $\lambda_{0}$ then read

$$
\begin{aligned}
& \mathbf{Q}\left(\lambda_{0}\right)=0, \\
& \mathcal{K}\left(\lambda_{0}\right)=(-1 \bmod 6,-1 \bmod 3,0 \bmod 2,0 \bmod 6,0 \bmod 3,0 \bmod 2,0 \bmod 2) .
\end{aligned}
$$




\begin{tabular}{c|c|rrr|c|c} 
Name & Monomial & $R_{1}$ & $R_{2}$ & $R_{3}$ & $k$ & $k n_{3}$ \\
\hline$\Omega_{1}^{\prime}$ & $\bar{X}_{0}^{c} S_{2} S_{5}$ & 0 & 0 & -1 & 6 & 0 \\
$\Omega_{2}^{\prime}$ & $\bar{X}_{0}^{c} X_{1}^{c} Y_{2}^{c}$ & -2 & -1 & 0 & 12 & 12 \\
$\Omega_{3}^{\prime}$ & $\bar{X}_{0}^{c}\left(S_{5}\right)^{2} U_{3}$ & -2 & 1 & -1 & 6 & 0 \\
$\Omega_{4}^{\prime}$ & $X_{0} \bar{X}_{1} S_{5} S_{7}$ & 0 & -1 & -1 & 6 & 3 \\
$\Omega_{5}^{\prime}$ & $X_{0} \bar{X}_{0}^{c} X_{1}^{c} \bar{X}_{1} U_{1}^{c}$ & -2 & -3 & 0 & 12 & 6 \\
$\Omega_{6}^{\prime}$ & $X_{0} \bar{X}_{0}^{c} \bar{X}_{1} \bar{Y}_{2}\left(S_{5}\right)^{2}$ & -2 & -1 & -1 & 12 & 6 \\
$\Omega_{7}^{\prime}$ & $X_{0} \bar{X}_{0}^{c} \bar{X}_{1} \bar{Y}_{2}\left(S_{6}\right)^{2}$ & 2 & -3 & -1 & 12 & 6 \\
$\Omega_{8}^{\prime}$ & $X_{0} \bar{X}_{0}^{c} X_{1}^{c} \bar{X}_{1} U_{2} U_{4}$ & -4 & -2 & 0 & 12 & 6
\end{tabular}

Table 20: Basis monomials of $\operatorname{ker} Q\left(\mathcal{S}_{2}\right)$ and their discrete charges. All monomials have $k n_{2}=k n_{2}^{\prime}=0$.

By subsequently adding singlets from the list (330), we find the maximal vacuum

$$
\mathcal{S}_{2}=\mathcal{S}_{0} \cup\left\{X_{1}^{c}, \bar{X}_{1}, Y_{2}^{c}, \bar{Y}_{2}, U_{1}^{c}, U_{3}, S_{6}, S_{7}\right\} .
$$

This vacuum contains 14 singlets. Its charge kernel has dimension eight, with basis monomials as given in Table 20. Again, the condition $R^{1}=-1 \bmod 6$ is obviously violated by all monomials in $\operatorname{ker} Q\left(\mathcal{S}_{2}\right)$. The vacuum is maximal, since the only possibility to enlarge it without breaking matter parity is to add singlets $A\left(A^{c}\right)$ whose $\mathcal{N}=2$ superpartners $A^{c}$ (A) already belong to $\mathcal{S}_{2}$. One then obtains the $\mu$-term

$$
\mu=\lambda_{0} \lambda_{s}, \quad \lambda_{0}=A A^{c}\left(\Omega_{5}^{\prime}\right)^{3},
$$

which is of order 16 in the singlets. Similar to the case of $\mathcal{S}_{1}$, going beyond the maximal vacuum leads to a generation of the $\mu$-term with large suppression. Order 16 may even be enough for the fulfillment of the phenomenological requirements.

The vacuum expectation value of the superpotential follows from monomials $\lambda_{0}$ with $\mathcal{K}\left(\lambda_{0}\right)=\mathcal{K}_{\text {vac }}$. Again, this condition cannot be fulfilled by the monomials from Table 20, since $\sum R^{1}$ is always even. Also for the case of partial gauge-Higgs unification one therefore has the property

$$
\langle W\rangle=\mu=0 \quad \text { to all orders, }
$$

as it is the case for full gauge-Higgs unification.

We now consider the mass term of the exotic pair of Higgs field candidates,

$$
W=m \Phi, \quad \Phi=\mathbf{5}_{1} \overline{\mathbf{5}}, \quad m=\lambda_{s} \lambda_{0},
$$

and choose $\lambda_{s}=\left(X_{1}\right)^{-1}$. One then obtains

$$
\mathcal{K}\left(\lambda_{s} \mathbf{5} \overline{\mathbf{5}}_{1}\right)=(0,0,-1,0,0,0,0) \quad \Rightarrow \quad m=0
$$




\begin{tabular}{c|c|c} 
Symmetry & $\alpha^{\prime}$ & $\mathbf{r}^{\prime}$ \\
\hline \hline$\left[\tilde{\mathbb{Z}}_{4}\right]_{R}$ & $\left(\frac{1}{2}, 0,-\frac{1}{12}, \frac{5}{8}, \frac{1}{24},-\frac{1}{30}\right)$ & $\left(\frac{1}{2}, 0,0,0,0,0,0\right)$ \\
{$\left[\mathbb{Z}_{2}\right]_{R}$} & $\left(-\frac{5}{2}, \frac{5}{2}, 0,-5,0, \frac{1}{2}\right)$ & $\left(0,0,0, \frac{5}{6}, 0,0,0\right)$ \\
\hline $\mathbb{Z}_{60}$ & $\left(\frac{1}{2}, \frac{1}{2},-\frac{1}{6}, \frac{1}{4}, \frac{1}{12}, \frac{1}{30}\right)$ & $(0,0,0,0,0,0,0)$
\end{tabular}

Table 21: The generators of the unbroken symmetry in the vacuum $\mathcal{S}_{2}$.

in the vacuum $\mathcal{S}_{2}$. This looks as if the situation is the same as for $\mathcal{S}_{1}$, where we found a two Higgs pair vacuum. However, there the full $2 \times 2$ mass matrix was zero, whereas here one finds

$$
W=\left\langle X_{1}^{c}\right\rangle \mathbf{5}_{1}\left(\overline{\mathbf{5}}+\epsilon \overline{\mathbf{5}}_{1}\right), \quad \epsilon=\left\langle X_{0} \bar{X}_{0}^{c} X_{1}^{c} Y_{2}^{c} S_{6} S_{7}\right\rangle .
$$

This shows that $\mathbf{5}_{1}$ decouples together with a linear combination of $\overline{\mathbf{5}}$ and $\overline{\mathbf{5}}_{1}$. The orthogonal linear combination is the down-type Higgs,

$$
H_{d}=\overline{\mathbf{5}}_{1}-\epsilon \overline{\mathbf{5}} \text {. }
$$

The vacuum $\mathcal{S}_{2}$ thus leads to a down-type Higgs with a dominant component from the second twisted sector, as it was already suggested by the Yukawa matrices (316) of the vacuum $\mathcal{S}_{0}$. In contrast, the up-type Higgs remains a pure gauge field in six dimensions, $H_{u}=\mathbf{5}$, giving an explanation of the large top-quark mass within our orbifold GUT model.

\section{Unbroken symmetries and interactions}

The unbroken symmetry of the vacuum $\mathcal{S}_{2}$ is

$$
G_{\text {vac }}\left(\mathcal{S}_{2}\right)=\left[\tilde{\mathbb{Z}}_{4} \times \mathbb{Z}_{2}\right]_{R} \times \mathbb{Z}_{60}
$$

with generators as shown in Table 21. Again, one obtains the factor $\mathbb{Z}_{60}$ which was also present in the vacuum $\mathcal{S}_{1}$. It has the generator $\frac{1}{2} t_{X}$, where $t_{X}$ is the generates $\mathrm{U}(1)_{X}$ in the false vacuum. This implies the presence of the matter parity from (300) in both studied vacua which extend $\mathcal{S}_{0}$. Dimension-four proton decay operators are therefore absent in both cases, however, dimension-five proton decay occurs also in the vacuum $\mathcal{S}_{2}$,

$$
C_{1111}^{(B)}=S_{6} S_{7} X_{1}^{c}\left(\bar{X}_{0}^{c}\right)^{2} \bar{X}_{1} Y_{2}^{c} .
$$

So far, there is no known model with vanishing $\mu$-term, interesting Yukawa couplings, preserved supersymmetry, no exotic particles, and a stable proton. In our setup, full proton stability seems to be linked to the existence of two Higgs pairs, as in the vacuum $\mathcal{S}_{0}$. We interpret the fact that we are not able to solve this problem as further need to understand the structure of the model, and maybe relate the question of decoupling to underlying smooth compactifications. We will comment more on this in the following chapter. 


\begin{tabular}{|c|c|c|c|c|c|}
\hline$i$ & $j$ & $Y_{i j}^{(d)}$ & $i$ & $j$ & $Y_{i j}^{(d)}$ \\
\hline 3 & 1 & $X_{0}\left(\bar{X}_{0}^{c}\right)^{2}\left(X_{1}^{c}\right)^{2} \bar{X}_{1} \bar{Y}_{2} U_{2} U_{4} S_{5}$ & 4 & 1 & $S_{5}$ \\
\hline 3 & 2 & $X_{0}\left(\bar{X}_{0}^{c}\right)^{2}\left(X_{1}^{c}\right)^{2} \bar{X}_{1} \bar{Y}_{2} U_{2} U_{4} S_{5}^{\prime}$ & 4 & 2 & $S_{5}^{\prime}$ \\
\hline 3 & 3 & $X_{0} X_{1}^{c} \bar{X}_{1} \bar{Y}_{2} S_{6} S_{7}$ & 4 & 3 & $S_{2} S_{5}$ \\
\hline 3 & 4 & $\bar{X}_{0}^{c}\left(X_{1}^{c}\right)^{2} Y_{2}^{c} S_{6} S_{7}$ & 4 & 4 & $\left(S_{5}\right)^{2}$ \\
\hline
\end{tabular}

Table 22: Examples of leading order monomials for the Yukawa couplings $Y_{i j}^{(d)}$ in the vacuum $\mathcal{S}_{2}$.

The $\mu$-term vanishes in the vacuum $\mathcal{S}_{2}$ by construction, we checked this explicitly by order-by-order scans up to order 500. The up-type Yukawa couplings are at lowest order given by the result $(315)$ for the vacuum $\mathcal{S}_{0}$. Hence we again benefit from partial gaugeHiggs unification by the generation of a large top-quark mass through the gauge interaction in the bulk. The down-type Yukawa couplings are given by

$$
C_{H_{d}=\overline{\mathbf{5}}_{1}}^{(d)}=\left(\begin{array}{llll}
0 & 0 & 0 & 0 \\
0 & 0 & 0 & 0 \\
s^{10} & s^{10} & s^{6} & s^{6} \\
s^{1} & s^{1} & s^{2} & s^{2}
\end{array}\right)
$$

with explicit lowest order monomials as in Table 22. Note that all zeros are protected by symmetries and thus present at arbitrary order in the singlets. This can be approved by close inspection of Table 31 in Appendix F. From the above matrix and the up-type analogue (315) one deduces the Yukawa couplings of the four-dimensional effective theory, as described in (313),

$$
W_{\text {Yuk }}=Y_{i j}^{(u)} u_{i}^{c} q_{j} H_{u}+Y_{i j}^{(d)} d_{i}^{c} q_{j} H_{d}+Y_{i j}^{(l)} l_{i} e_{j}^{c} H_{d},
$$

with

$$
Y^{(u)}=\left(\begin{array}{ccc}
s^{4} & s^{4} & s^{5} \\
s^{4} & s^{4} & s^{5} \\
s^{5} & s^{5} & g
\end{array}\right), \quad Y^{(d)}=\left(\begin{array}{ccc}
0 & 0 & 0 \\
0 & 0 & 0 \\
s^{1} & s^{1} & s^{2}
\end{array}\right), \quad Y^{(l)}=\left(\begin{array}{ccc}
0 & 0 & 0 \\
0 & 0 & 0 \\
s^{10} & s^{10} & s^{6}
\end{array}\right)
$$

This explicitly shows that the top-quark mass is proportional to the gauge coupling $g$ in six dimensions. The lepton and down-quark mass matrices do not look convincing. In fact, they predict $m_{e}=m_{\mu}=m_{d}=m_{s}=0$, which excludes the studied model. One can now either understand this as a problem of the specific choice of gauge embedding that we chose and study other possibilities, as claimed in [14]. An alternative approach may be to further explore correlations between the decoupling of exotics and the identification of matter and Higgs fields, and the generated interactions. Possibly the details of the physical vacuum are closely linked to the dynamics of the underlying geometry, once the restriction to the orbifold point is relaxed. 


\subsection{The all-order superpotential}

So far symmetry arguments were given for the vanishing of couplings to all orders in the singlets. This was then used as a tool for the construction of partly-realistic vacua. The non-vanishing couplings were calculated by order-by-order scans over all monomials within a given vacuum $\mathcal{S}$. Here we argue that the methods introduced in Section 4.6 can be extended to an algorithm which allows for the calculation of the superpotential to all orders, without relying on the naive order-by-order approach.

In (321) we separated the continuous and the discrete transformation behavior of a superpotential term $\lambda \Phi$, where the monomial $\lambda=s_{1} \cdots s_{N}, s_{i} \in \mathcal{S}$, determines the coupling of the interaction $\Phi=\phi_{1} \cdots \phi_{M}, \phi_{i} \notin \mathcal{S}$,

$$
W \supset \lambda_{0} \lambda_{s}^{\Phi} \Phi, \quad \mathbf{Q}\left(\lambda_{s}^{\Phi} \Phi\right)=\mathbf{Q}\left(\lambda_{0}\right)=0, \quad \mathcal{K}\left(\lambda_{0}\right)=\mathcal{K}_{\mathrm{vac}}-\mathcal{K}\left(\lambda_{s} \Phi\right) .
$$

In fact, the total superpotential is a sum of such terms, which we did not write out explicitly. It will have an overall factor which is completely invariant under all symmetries. The corresponding terms, written as $\omega_{0} \in \operatorname{ker} Q(\mathcal{S})$, are then universal contributions to $\lambda_{0}$,

$$
\lambda_{0}=\omega_{0} \lambda_{0}^{\Phi}, \quad \mathcal{K}\left(\lambda_{0}^{\Phi}\right)=\mathcal{K}_{\mathrm{vac}}-\mathcal{K}\left(\lambda_{s}^{\Phi} \Phi\right), \quad \mathcal{K}\left(\omega_{0}\right)=\mathcal{K}_{0},
$$

where

$$
\mathcal{K}_{0}=(0 \bmod 6,0 \bmod 3,0 \bmod 2,0 \bmod 6,0 \bmod 3,0 \bmod 2,0 \bmod 2) .
$$

We denote the defining properties of $\omega_{0}$ as $\omega_{0} \in \operatorname{ker} Q \cap \operatorname{ker} \mathcal{K}$. The latter set of monomials can now be calculated for a given vacuum configuration $\mathcal{S}$. Since the $\omega_{0}$ are universal, this has to be done only once for the full superpotential.

However, what remains to be found for each interaction $\Phi$ are the monomials $\lambda_{s}^{\Phi} \in \mathcal{S}$ and $\lambda_{0}^{\Phi} \in \operatorname{ker} Q(\mathcal{S})$. If one can find a basis of $\operatorname{ker} Q \cap \operatorname{ker} \mathcal{K}$, it is sufficient to find only one solution. In the end, the projection (323) to physical monomials with positive exponents has to be evaluated. The total superpotential to all orders can then be written as

$$
W=\mathcal{P}_{\mathbb{N}}\left(\sum_{\omega_{0}} \omega_{0}\right)\left(\sum_{\Phi} \lambda_{0}^{\Phi} \lambda_{s}^{\Phi} \Phi\right),
$$

where the omnipresent and unknown coefficients are not shown.

Recall from Section 3.1.2 that the discrete symmetries discussed so far are not sufficient for the description of all interactions of the effective theory. There we found that is was necessary to discuss the terms which contain only fields from equivalent twisted sectors separately. They can be collected into a part $W_{0}$ of the full superpotential,

$$
W_{\text {tot }}=W+W_{0},
$$

which is then subject to additional $\mathbb{Z}_{6}^{n_{6} k}$ symmetry constraints. If such terms appear in the potential (358) the corresponding condition

$$
\sum n_{6} k=0 \bmod 6
$$

has to be checked a posteriori. 


$$
\begin{array}{l|l}
\tilde{\Omega}_{1} & \left(S_{2}\right)^{-1} X_{0}\left(X_{1}\right)^{-1} \bar{X}_{1}\left(Y_{2}\right)^{-1} S_{7} \\
\tilde{\Omega}_{2} & \left(S_{5}\right)^{2}\left(S_{2}\right)^{2}\left(\bar{X}_{0}^{c}\right)^{2} \\
\tilde{\Omega}_{3} & \left(U_{4}\right)^{-2}\left(U_{2}\right)^{-2}\left(S_{5}\right)^{2}\left(S_{2}\right)^{-2}\left(X_{0}\right)^{2}\left(\bar{X}_{0}^{c}\right)^{-2}\left(\bar{X}_{1}\right)^{2}\left(Y_{2}\right)^{-2}\left(S_{7}\right)^{4} \\
\tilde{\Omega}_{4} & \left(S_{2}\right)^{-6}\left(X_{0}\right)^{6}\left(\bar{X}_{0}^{c}\right)^{-3}\left(X_{1}\right)^{-3}\left(\bar{X}_{1}\right)^{6}\left(Y_{2}\right)^{-3}\left(S_{7}\right)^{6}
\end{array}
$$

Table 23: Basis monomials of $\operatorname{ker} Q \cap \operatorname{ker} \mathcal{K}$ for the vacuum $\mathcal{S}_{1}$.

\section{Example: The vacuum $\mathcal{S}_{1}$}

As an example for the algorithm suggested above by (358), we calculate the superpotential for the vacuum $\mathcal{S}_{1}$ from (284). The corresponding basis monomials of the charge kernel were given in Table 16 . The basis of $\operatorname{ker} Q \cap \operatorname{ker} \mathcal{K}$ can then be found by calculating

$$
\operatorname{ker}\left(\begin{array}{rrrrrrrrr}
0 & 0 & 0 & -3 & 6 & 0 & 0 & 0 & 0 \\
0 & -1 & -1 & -2 & 0 & 3 & 0 & 0 & 0 \\
-1 & -1 & -1 & 0 & 0 & 0 & 2 & 0 & 0 \\
6 & 6 & 6 & 6 & 0 & 0 & 0 & 6 & 0 \\
0 & 6 & 3 & 6 & 0 & 0 & 0 & 0 & 3
\end{array}\right)
$$

where the first four columns contain the quantum numbers $R^{1}, R^{2}, R^{3}, k, n_{3} k$ of the four basis monomials $\Omega_{i}$ of $\operatorname{ker} Q\left(\mathcal{S}_{1}\right)$, and the last five columns are added in order to find solutions modulo the corresponding order, as given in (357). This yields a basis $\mathcal{B}_{0}$ for $\operatorname{ker} Q \cap \operatorname{ker} \mathcal{K}$,

$$
\mathcal{B}_{0}=\{\underbrace{\left(\Omega_{2}\right)^{-1} \Omega_{3}}_{\tilde{\Omega}_{1}}, \underbrace{\left(\Omega_{1}\right)^{2}}_{\tilde{\Omega}_{2}}, \underbrace{\left(\Omega_{1}\right)^{-2}\left(\Omega_{3}\right)^{4}\left(\Omega_{4}\right)^{-2}}_{\tilde{\Omega}_{3}}, \underbrace{\left(\Omega_{1}\right)^{-3}\left(\Omega_{2}\right)^{-3}\left(\Omega_{3}\right)^{6}}_{\tilde{\Omega}_{4}}\} .
$$

The monomials $\tilde{\Omega}_{i}$ are then completely invariant under the continuous and the discrete symmetries. Their explicit form is given in Table 23. Note the appearance of inverse exponents, which will eventually be subject to the projection $\mathcal{P}_{\mathbb{N}}$. Consider for example the down-type Yukawa coupling

$$
\Phi=\overline{\mathbf{5}}_{(4)} \mathbf{1 0} \mathbf{0}_{(4)} \overline{\mathbf{5}}_{1}
$$

for which one finds

$$
\lambda_{s}^{\Phi}=S_{5}\left(S_{2}\right)^{-1}\left(\bar{X}_{0}^{c}\right)^{-1}, \quad \lambda_{0}^{\Phi}=\left(\Omega_{1}\right)^{-1}=\left(S_{2}\right)^{-1}\left(S_{5}\right)^{-1}\left(\bar{X}_{0}^{c}\right)^{-1} .
$$

Table 23 shows that the smallest term with only positive exponents arises for $\omega_{0}=\tilde{\Omega}_{2}$ and the corresponding coupling is

$$
C_{44}^{(d)}=\tilde{\Omega}_{2} \lambda_{0}^{\Phi} \lambda_{s}^{\Phi}=\left(S_{5}\right)^{2},
$$

in agreement with (316). The next terms then follow from

$$
\omega_{0}=\left(\tilde{\Omega}_{2}\right)^{2},\left(\tilde{\Omega}_{1}\right)^{-3}\left(\tilde{\Omega}_{2}\right)^{2} \tilde{\Omega}_{4},\left(\tilde{\Omega}_{1}\right)^{-4}\left(\tilde{\Omega}_{2}\right)^{2} \tilde{\Omega}_{4},\left(\tilde{\Omega}_{1}\right)^{-5}\left(\tilde{\Omega}_{2}\right)^{2} \tilde{\Omega}_{4},\left(\tilde{\Omega}_{1}\right)^{-6}\left(\tilde{\Omega}_{2}\right)^{2} \tilde{\Omega}_{4},
$$


where the last four choices all produce couplings of order 13.

The evaluation of the projection to physical monomials corresponds to solving Diophantine equations. We find that for the ten-field vacuum $\mathcal{S}_{1}$, the algorithm (358) can be solved to very high orders without any restrictions due to limits of computing power (order 50 in less than a minute). We expect that the efficiency changes if one studies much larger vacua. Here, the method correctly reproduces the Yukawa matrices (315), (316) and higher order terms, which we do not explicitly list.

\subsubsection{The calculation of $\boldsymbol{F}$-terms}

The method (358) can also be used for the calculation of $F$-terms $F_{u}=\partial W / \partial u$, by choosing $\Phi=u \notin \mathcal{S}$. The expectation value of $F_{u}$ only gets non-zero contributions from terms in which $u$ is the only field in the monomial which is not contained in $\mathcal{S}$,

$$
W=\lambda u, \quad \lambda=s_{1} \cdots s_{N}, \quad s_{i} \in \mathcal{S} .
$$

Now consider $u$ from the second or the fourth twisted sector and the case that the vacuum $\mathcal{S}$ contains the charge conjugate field $u^{c}$. For the maximal vacuum $\mathcal{S}_{2}$, this is the case for all $u$ with non-trivial $F$-terms. Then one can choose

$$
\lambda_{s}^{u}=u^{c}, \quad \lambda_{0}^{u}=\Omega_{0} \in \operatorname{ker} Q, \quad \mathcal{K}\left(\Omega_{0} u^{c} u\right)=\mathcal{K}_{\mathrm{vac}},
$$

with a universal monomial $\Omega_{0}$. For the vacua $\mathcal{S}_{1}, \mathcal{S}_{2}$ this is the monomial which was used for the universal decoupling, $\Omega_{0}=S_{2} S_{5} \bar{X}_{0}^{c}$. With (358) the $F$-terms take the form

$$
F_{u}=\mathcal{P}_{\mathbb{N}}\left(\sum_{\omega_{0}} \omega_{0}\right) \Omega_{0} u^{c},
$$

up to coefficients. Due to $u^{c} \in \mathcal{S}$ the conditions $F_{u}=0$, required for unbroken supersymmetry, are not universal. The projection to physical monomials depends on $u^{c}$ and has to be evaluated for each case separately. We do not perform this calculation here, and simply assume the existence of solutions in the studied vacua, which are needed for consistency of the model.

\section{Connection with smooth geometries}

In the previous chapters, we have described in detail an effective local orbifold GUT model in six dimensions, obtained from an anisotropic compactification of the heterotic string. It was found by first compactifying four internal dimensions on an orbifold $T^{4} / \mathbb{Z}_{3}$, whose volume was assumed to be small, the other two on a torus of GUT scale size, and then dividing out another $\mathbb{Z}_{2}$ symmetry. This short chapter is meant to be an outlook on work in progress, concerned with the possibility that the four small internal dimensions are not 
compactified on an orbifold, but on the smooth manifold $K 3$. The orbifold $T^{4} / \mathbb{Z}_{3}$ is a singular limit of the latter, and thus a comparison of the two compactifications,

$$
\frac{T^{4} / \mathbb{Z}_{3} \times T^{2}}{\mathbb{Z}_{2}} \text { versus } \frac{K 3 \times T^{2}}{\mathbb{Z}_{2}},
$$

may yield insights on the role of the fields that were obtained on the orbifold side. Note that this idea is somehow diametrical to the standard blow-up approach (cf. [40,51-56]). We hope that the above comparison may help to improve the understanding of the decoupling procedure and the structure of physical vacua. Here we present first results in this direction $^{28}$.

\subsection{Compactification on the $K 3$ surface}

The $K 3$ surface is a standard example for string compactifications on a manifold (see for example $[76,83])$. It is the only non-trivial Calabi-Yau in two complex dimensions. Its topology has Euler characteristic $\chi=24$, corresponding to the result

$$
\frac{1}{16 \pi^{2}} \int_{K 3} \operatorname{tr} R^{2}=24
$$

This has to be understood together with the 'tadpole cancelation condition',

$$
\int_{K 3}\left(\operatorname{tr} R^{2}-\operatorname{tr} F^{2}\right)=0
$$

which is required for the absence of anomalies, cf. Section 3.3. The above formulas imply that consistent compactifications on $K 3$ are tied to the existence of a non-vanishing gauge background. This necessarily breaks the $\mathrm{E}_{8} \times \mathrm{E}_{8}$ gauge group, and the possible effective theories that can be obtained in six dimensions correspond to the different choices for the embedding of the broken generators into the full gauge group [53,76,84]. The non-vanishing gauge background of the compactified theory is then often referred to as the 'gauge bundle' in the literature.

The topological invariant on the gauge theory side is called the 'instanton number', and as a consequence of the tadpole cancelation condition it also has to sum to 24 . The breaking of the gauge group can then be characterized by the distribution of the instantons among subgroups of $\mathrm{E}_{8} \times \mathrm{E}_{8}$. If they all sit in a subgroup $H$, its commutant $G$ gives the unbroken gauge symmetry, defined such that $G \times H$ is a maximal subgroup of the original symmetry.

Both the geometry and the gauge symmetry background are associated with moduli fields, which comprise the degrees of freedom that describe fluctuations around that background. Including fluctuations of the antisymmetric tensor field and the volume, the geometrical part has a moduli space of dimension 80 and is essentially given by [83]

$$
\frac{O(4,20)}{O(4) \times O(20)} \Rightarrow 80 \text { degrees of freedom } \sim 20 \text { hypermultiplets. }
$$

\footnotetext{
${ }^{28}$ We are grateful to Taizan Watari for related discussions.
} 
For the gauge bundles, the moduli space depends on the model. We refer the reader to table 3 of [84] for a classification of various possibilities to break $E_{8} \times E_{8}$ to a subgroup, and focus only on one particular case of interest for the comparison with the orbifold model discussed in this work. In Chapter 3 the gauge group and the matter content of the six-dimensional bulk theory were found to be

$$
\begin{aligned}
T^{4} / \mathbb{Z}_{3}: \quad & \mathrm{SU}(6) \times \mathrm{U}(1)^{3} \times\left[\mathrm{SU}(3) \times \mathrm{SO}(8) \times \mathrm{U}(1)^{2}\right] \\
& (\mathbf{2 0}, \mathbb{1}, \mathbb{1})+9 \cdot[(\mathbf{6}, \mathbb{1}, \mathbb{1})+(\overline{\mathbf{6}}, \mathbb{1}, \mathbb{1})]+9 \cdot[(\mathbb{1}, \mathbf{3}, \mathbb{1})+(\mathbb{1}, \overline{\mathbf{3}}, \mathbb{1})] \\
& +4 \cdot\left[(\mathbb{1}, \mathbb{1}, \mathbf{8})+\left(\mathbb{1}, \mathbb{1}, \boldsymbol{8}_{s}\right)+\left(\mathbb{1}, \mathbb{1}, \boldsymbol{8}_{c}\right)\right]+42 \cdot(\mathbb{1}, \mathbb{1}, \mathbb{1}) .
\end{aligned}
$$

In the following we shall compare this model to a compactification on $K 3$ which yields the following gauge symmetry:

$$
\text { K3: } \quad \mathrm{SU}(6) \times[\mathrm{SO}(8)] .
$$

We thus assume that all U(1) factors as well as the hidden sector SU(3) gauge group are remnants of the singular orbifold limit in the geometrical moduli space.

The corresponding instanton distribution is $[84,85]$

$$
\begin{array}{ll}
\text { Visible sector: } & \mathrm{E}_{8} \supset \mathrm{SU}(6) \times \underbrace{\langle\mathrm{SU}(2)\rangle}_{6 \text { instantons }} \times \underbrace{\langle\mathrm{SU}(3)\rangle}_{6 \text { instantons }}, \\
\text { Hidden sector: } & \mathrm{E}_{8} \supset \mathrm{SO}(8) \times \underbrace{\langle\mathrm{SO}(8)\rangle}_{12 \text { instantons }} .
\end{array}
$$

From these numbers one can also infer the number of massless matter multiplets in the effective theory in six dimensions. The result is

$$
K 3: \quad(\mathbf{2 0}, \mathbb{1})+9 \cdot[(\mathbf{6}, \mathbb{1})+(\overline{\mathbf{6}}, \mathbb{1})]+4 \cdot\left[(\mathbb{1}, \mathbf{8})+\left(\mathbb{1}, \mathbf{8}_{s}\right)+\left(\mathbb{1}, \mathbf{8}_{c}\right)\right],
$$

and perfectly matches the spectrum of the orbifold result (375), as far as the common gauge group $\mathrm{SU}(6) \times[\mathrm{SO}(8)]$ is concerned. The discrepancies will be discussed shortly.

The instanton numbers also specify the gauge bundle moduli space. The corresponding degrees of freedom can be understood in terms of hypermultiplets of the six-dimensional effective theory:

$$
\begin{array}{ll}
\text { SU(2) gauge bundle: } & 9 \text { hypermultiplets, } \\
\text { SU(3) gauge bundle: } & 10 \text { hypermultiplets, } \\
\text { SO(8) gauge bundle: } & 44 \text { hypermultiplets. }
\end{array}
$$

In summary, the $K 3$ compactification with the above gauge bundles, to which we want to compare the orbifold results, has a smaller unbroken gauge group and matter spectrum, but predicts 83 moduli hypermultiplets,

$$
\text { K3 : } \quad \underbrace{19}_{\text {vis. } \mathrm{E}_{8}}+\underbrace{44}_{\text {hid. } \mathrm{E}_{8}}+\underbrace{20}_{\text {geom. }}=83 \text { moduli. }
$$


On the other hand, the orbifold provides a larger gauge group and additional matter transforming under the latter, as well as 40 singlets and two geometrical moduli hypermultiplets, denoted as $C_{1}, C_{2}$ in (155). However, additionally there are non-zero Fayet-Iliopoulos terms (272), and parts of the gauge symmetry will be broken. As will be described in the following, this breaking is the key for a successful matching of the two approaches.

\section{$5.2 \quad$ Identifying moduli}

The 320 hypermultiplets (375) of the $T^{4} / \mathbb{Z}_{3}$ compactification arise from different sectors of the orbifold,

$$
\begin{aligned}
& \left.\begin{array}{l}
3\left[(\mathbb{1}, \mathbb{1}, \mathbf{8})+\left(\mathbb{1}, \mathbb{1}, \mathbf{8}_{s}\right)+\left(\mathbb{1}, \mathbb{1}, \mathbf{8}_{c}\right)\right] \\
3 \cdot 3 \cdot[(\mathbf{6}, \mathbb{1}, \mathbb{1})+(\overline{\mathbf{6}}, \mathbb{1}, \mathbb{1})]
\end{array}\right\} \quad k=2,4, \\
& 3 \cdot 3 \cdot[(\mathbb{1}, \mathbf{3}, \mathbb{1})+(\mathbb{1}, \overline{\mathbf{3}}, \mathbb{1})]\} \quad \text { no oscillators, } \\
& 3 \cdot 3 \cdot[(\mathbb{1}, \mathbb{1}, \mathbb{1})+(\mathbb{1}, \mathbb{1}, \mathbb{1})]\} \\
& \underbrace{3}_{\gamma=0, \frac{1}{2}, 1} \cdot \underbrace{3}_{n_{3}=0,1,2} \cdot[(\mathbb{1}, \mathbb{1}, \mathbb{1})+(\mathbb{1}, \mathbb{1}, \mathbb{1})]: \quad \begin{array}{l}
k=2,4, \\
\text { non-zero oscillator numbers, }
\end{array} \\
& \left.\begin{array}{r}
(\mathbb{1}, \mathbb{1}, \mathbf{8})+\left(\mathbb{1}, \mathbb{1}, \mathbf{8}_{s}\right)+\left(\mathbb{1}, \mathbb{1}, \mathbf{8}_{c}\right) \\
(\mathbf{2 0}, \mathbb{1}, \mathbb{1})+4 \cdot(\mathbb{1}, \mathbb{1}, \mathbb{1})
\end{array}\right\} \quad \text { untwisted sector, charged, } \\
& 2 \cdot(\mathbb{1}, \mathbb{1}, \mathbb{1}): \quad \quad \text { untwisted sector, uncharged. }
\end{aligned}
$$

The singlets in (382b) carry non-vanishing oscillator numbers $\mathbf{N}, \mathbf{N}^{*}, \tilde{\mathbf{N}}, \tilde{\mathbf{N}}^{*}$, defined in (52). The singlets in (382c) and (382d) were called $U_{1}, \ldots, U_{4}$ and $C_{1}, C_{2}$ in the previous chapters, respectively. The two latter hypermultiplets play the role of geometrical moduli of the orbifold geometry.

We can only make contact with the assumed $K 3$ configuration, if we also break the additional gauge group factors $\mathrm{U}(1)^{3} \times\left[\mathrm{SU}(3) \times \mathrm{U}(1)^{2}\right]$ on the orbifold side by a Higgs mechanism. This means that some of the degrees of freedom in the above list are traded for masses of the corresponding gauge bosons and gauginos,

$$
\begin{aligned}
\mathrm{U}(1)^{3}: & 3 \text { hypermultiplets, } \\
\mathrm{SU}(3) \times \mathrm{U}(1)^{2}: & 8+2=10 \text { hypermultiplets. }
\end{aligned}
$$

Let us now count the degrees of freedom that remain on the orbifold side. We are left with $\mathrm{SU}(6) \times[\mathrm{SO}(8)]$ gauge symmetry after the Higgs mechanism. From the representations of the hidden sector $\mathrm{SU}(3)$ one obtains $9 \cdot(3+3)=54$ hypermultiplets. For the visible sector, one can show that for each localization quantum number $n_{3}=0,1,2$, the multiplets from (382a) arise from different embeddings of the group $\mathrm{SO}(14)$ into $\mathrm{E}_{8} \times \mathrm{E}_{8}$. This fact was observed in [13] and not stressed much in the previous chapters. For each value of $n_{3}$ and $\gamma$ one has the decomposition $\mathbf{1 4}=\mathbf{6}+\overline{\mathbf{6}}+2 \cdot \mathbb{1}$, and we can associate $3 \cdot 3 \cdot 2=18$ singlets with vanishing oscillator numbers with the visible sector. Amending the latter by the four 
charged untwisted singlets, one obtains after the Higgs mechanism

$$
\begin{aligned}
& \text { Visible sector : } \quad 18+4-3=19 \quad \text { hypermultiplets, } \\
& \text { Hidden sector : } \quad 54-10=44 \quad \text { hypermultiplets . }
\end{aligned}
$$

The degrees of freedom from the twisted sectors of the orbifold match the gauge bundle moduli in (381) of the $K 3$ compactification, after breaking the additional gauge symmetry.

Furthermore, 18 twisted singlets from oscillator excitations in the internal directions are present on the orbifold, as well as two from the untwisted sector with the same property. Together, they may correspond to the geometrical moduli of $K 3$ :

$$
\text { Oscillator excitations: } \quad 18+2=20 \quad \text { hypermultiplets . }
$$

We have thus found a correspondence between the gauge bundle moduli and the geometrical moduli of a smooth $K 3$ compactification and the hypermultiplets of the orbifold, after spontaneous breakdown of the additional symmetries.

It is a remarkable fact that the hypermultiplets which play the role of geometrical moduli are charged under the U(1)'s of the orbifold. This may be related to the fact that we chose a gauge background on $K 3$ without Abelian factors and therefore they are specific to the orbifold point. This issue and the interpretation of the above is still under debate.

\subsection{Outlook: A local GUT based on $K 3$}

The next step would be to complete the comparison (370) by defining the action of $\mathbb{Z}_{2}$ on the manifold $K 3$ and work out the local spectra at the fixed points of $T^{2} / \mathbb{Z}_{2}$. The involutions on $K 3$ were classified in [86] and their application to our model is work in progress. We hope that the comparison with the results obtained for the local orbifold GUT may lead to significant progress in understanding the validity of the orbifold description and its interpretation in terms of their smooth counterparts.

\section{Conclusions}

Field theoretical orbifold GUTs can be derived from heterotic string theory by the consideration of anisotropic compactifications. We explicitly constructed an effective $T^{2} / \mathbb{Z}_{2}$ model in six dimensions with some interesting properties. For vanishing vacuum expectation values, it has $\mathrm{SU}(6)$ gauge symmetry in the bulk, which is broken to the GUT group $\mathrm{SU}(5)$ at two fixed points of the geometry. There, two complete standard model families are localized and it was possible to uniquely determine a $\mathrm{U}(1)_{X}$ gauge symmetry, which combines with the hypercharge to a unique $\mathrm{U}(1)_{B-L}$ in the effective four-dimensional theory. This was then used as a tool to interpret the bulk fields of the model, with the result that two additional complete quark-lepton generations in the SU(5) language could be identified. They are split multiplets, yielding the missing third standard model family in four dimensions as the sum of their zero modes. This as such is an interesting setup for a higher 
dimensional GUT model, since it resembles the well-known and successful orbifold solution of the doublet-triplet splitting problem of the GUT Higgs multiplets in the matter sector. Phenomenologically, this mechanism avoids the problematic mass relations obtained in standard SU(5) GUTs in four dimensions. However, also the successful predictions are lost, and the effective Yukawa matrices are to a high degree vacuum dependent.

In fact, this statement generalizes to all superpotential interactions. A main point that we learn from the explicit calculation of the spectrum is therefore that a better understanding of the vacuum structure of orbifold models is required in order to reveal the mechanisms of string theory behind phenomenological observations, if existent.

Closely linked to that issue is the problem of the presence of exotic fields in the model, which are unavoidable in the false vacuum. Since they are vector-like, it is possible to generate mass terms for them which imply their decoupling from the effective low-energy theory. However, this is inevitably linked to the simultaneous generation of interactions, since the total superpotential originates from the sum of all consistent higher-dimensional operators. The latter are restricted by continuous gauge symmetries and a set of discrete symmetries. We explicitly calculated these symmetries for example vacua and found that they explain the absence or presence of interactions in the effective superpotential. This provides a tool for reversing the order of the construction of phenomenologically interesting vacua: Instead of throwing dice and calculating the results, it is possible to construct vacua such that they allow or forbid specific terms of phenomenological interest. Furthermore, we found an algorithm which allows the calculation of the superpotential to arbitrary order in the singlets with better efficiency than the naive order-by-order approach.

All exotics at the SU(5) GUT fixed points are bulk fields in the studied model, almost all arise from the second or the fourth twisted sectors of the $\mathbb{Z}_{6-\text { II }}$ orbifold. Due to the existence of inequivalent superpositions of states from equivalent Hilbert spaces, the latter hypermultiplets have an overall multiplicity of three in the bulk. The intertwining of gauge and supersymmetry breaking at the orbifold fixed points then allows for the universal decoupling of many exotics, since uncharged pairs of fields with different superposition quantum numbers can be formed. This mechanism is independent of the specific gauge embedding of the model.

This universal decoupling required only four singlet fields with a non-zero vacuum expectation value. We extended this set to two different maximal vacua, which cannot be enlarged further without either violating matter parity or generating a $\mu$-term. This showed that in the studied model, the unification of both the up- and the down-type Higgs with gauge fields of the six-dimensional bulk is impossible. Instead, we found that either a model with two Higgs pairs can be realized, which is incompatible with gauge coupling unification, or partial gauge Higgs unification. The latter corresponds to the situation that only the up-type Higgs is contained in the gauge vector, with the desirable prediction of a large top-quark mass. In fact, the case of full gauge-Higgs unification is phenomenologically not preferred over the partial gauge-Higgs unification scenario. One may even argue that the symmetry between the fundamental and the anti-fundamental representation of $\mathrm{SU}(5)$ is already broken by the identification of matter with the latter. However, both vacua predict massless electrons and down-quarks and are thus excluded as physical vacua. 
This observation is not surprising. The whole analysis was based on one particular model, contained in a whole landscape of possibilities. The motivation for the work was not to find the standard model vacuum by pure luck, but to consider one model in detail and gain a better understanding of the problems that form the typical obstacles on the way towards fully realistic vacua.

The main part of this paper was concerned with the issue of vacuum selection and the question of relating the latter to phenomenology. Another source of problems may arise from the misinterpretation of remnants of the orbifold construction. It is apparent from the calculation of anomalies that the effective orbifold GUT model has localized Fayet-Iliopoulos terms of GUT scale at the fixed points. Thus even though we could show explicitly that all anomalies of the effective orbifold field theory vanish or can be canceled, the trivial vacuum is inconsistent, since it is not supersymmetric. This implies that singlet fields acquire large vacuum expectation values during the transition to the supersymmetric solution, thereby blowing up singularities. The orbifold description can thus only be effective, and its validity as an approximate description is not clear a priori. For the bulk theory of the effective orbifold GUT in six dimensions it was possible to identify the fields of the orbifold with the moduli of a compactification of four dimensions of heterotic string theory on the $K 3$ manifold. This assigns new interpretations to the singlets of the orbifold model. One may hope that the analogue orbifold GUT can be constructed in terms of an anisotropic compactification, where the small dimensions are compactified on $K 3$, modulo an involution. If the resulting model can also be matched with the $\mathbb{Z}_{6-\text { II }}$ orbifold case, new insights on the validity and interpretation of orbifolds may be gained, including new guiding principles for the search for physical vacua.

\section{Acknowledgements}

I would like to thank Wilfried Buchmüller, Rolf Kappl, Christoph Lüdeling, Jan Möller, Saúl Ramos-Sánchez, Kai Schmidt-Hoberg and Hagen Triendl for many valuable discussions and comments.

\section{A Orbifold details}

\section{A.1 Solving the fixed point equation}

For any element $g=\left(\theta^{k}, m_{a} \mathbf{e}_{a}\right) \in S$ one can write down the fixed point condition

$$
(\mathbb{1}-g) \mathbf{Z}_{g}=0
$$

Thus the corresponding fixed point or fixed plane coordinates form the kernel of $(\mathbb{1}-g)$. They can be expressed as

$$
\left(1-\vartheta_{(i)}^{k}\right) Z_{g}^{i}=\pi m_{a} e_{a}^{i} .
$$


This equation has no solution if $g$ describes a pure translation in the plane $i$, which means $\vartheta_{(i)}^{k}=1, m_{a} e_{a}^{i} \neq 0$. Otherwise the solutions are given by

$$
Z_{g}^{i}= \begin{cases}\left(1-\vartheta_{(i)}^{k}\right)^{-1} \pi m_{a} e_{a}^{i}, & \text { if } \vartheta_{(i)}^{k} \neq 1, \\ \text { unconstrained, } & \text { if } \vartheta_{(i)}^{k}=1, m_{a} e_{a}^{i}=0 .\end{cases}
$$

Here the first line describes a localization in the plane $i$, while for non-trivial $g$ the latter corresponds to a fixed plane solution.

In summary, the space group $S$ splits into two regimes. One generates winding modes in at least one of the planes $\left(Z^{i} \rightarrow Z^{i}+m_{a} e_{a}^{i} \neq Z^{i}\right)$. All other elements fulfill a fixed pointed equation. These regimes can now be decomposed into disjoint conjugacy classes, yielding a non-redundant description of the space group.

\section{A.2 Space group selection rules for $\mathbb{Z}_{6-I I}$}

A coupling

$$
W=\alpha \phi_{1} \cdots \phi_{M}
$$

of $M$ multiplets at fixed points with space group elements $g_{(l)}=\left(\theta^{k_{(l)}}, m_{a}^{(l)}\right), l=1, \ldots, M$, can be present if the space group selection rule (100) is fulfilled,

$$
\sum_{l} m_{a}^{(l)} \mathbf{e}_{a}=0 \bmod \sum_{l} \Lambda_{k_{(l)}}
$$

The sub-lattices $\Lambda_{k}$ are given in Table 3 . We count the number of multiplets $\phi_{l}$ with quantum numbers $k, n_{i}$ by $N_{k, n_{i}}$, where $n_{i} \equiv n_{6}, n_{3}$ and $n_{i} \equiv n_{2}, n_{2}^{\prime}$ in the $G_{2}, \mathrm{SU}(3)$ - and the SO(4)-plane, respectively. With Table 2 the left hand side of (391) then gives for the three planes

$$
\begin{aligned}
G_{2}: \quad \sum_{l} m_{a}^{(l)} \mathbf{e}_{a}= & \left(N_{3,1}+N_{2,2}\right) \mathbf{e}_{1}+\left(N_{3,1}+N_{2,2}-N_{4,2}\right) \mathbf{e}_{2} \\
\mathrm{SU}(3): \quad \sum_{l} m_{a}^{(l)} \mathbf{e}_{a}= & \left(N_{1,2}+N_{2,1}+N_{2,2}+N_{4,2}+N_{5,1}+N_{5,2}\right) \mathbf{e}_{3} \\
& +\left(N_{1,1}+N_{1,2}+N_{2,1}+N_{4,1}+N_{4,2}+N_{5,1}\right) \mathbf{e}_{4}, \\
\mathrm{SO}(4): \quad \sum_{l} m_{a}^{(l)} \mathbf{e}_{a}= & \left(N_{1,1,0}+N_{1,1,1}+N_{3,1,0}+N_{3,1,1}+N_{5,1,0}+N_{5,1,1}\right) \mathbf{e}_{5} \\
& +\left(N_{1,0,1}+N_{1,1,1}+N_{3,0,1}+N_{3,1,1}+N_{5,0,1}+N_{5,1,1}\right) \mathbf{e}_{6} .
\end{aligned}
$$

The $\mathrm{G}_{2}$-plane was discussed in Section 3.1.2 of the main text. Recall from table 3 that the sub-lattices relevant for the $\mathrm{SU}(3)$ - and the $\mathrm{SO}(4)$-plane are

$$
\begin{array}{ll}
\mathrm{SU}(3): & \left\{a \mathbf{e}_{1}+3 b \mathbf{e}_{2} \mid a, b \in \mathbb{Z}\right\} \subset \Lambda_{2}, \\
\mathrm{SO}(4): & \left\{2 a \mathbf{e}_{5}+2 \mathbf{e}_{6} \mid a, b \in \mathbb{Z}\right\} \subset \Lambda_{3} .
\end{array}
$$


The condition (391) can thus be evaluated as

$$
\begin{aligned}
& \mathrm{SU}(3): \quad 2\left(N_{1,2}+N_{2,1}+N_{4,2}+N_{5,1}\right)+N_{1,1}+N_{2,2}+N_{4,1}+N_{5,2}=0 \bmod 3, \\
& \mathrm{SO}(4): \quad N_{1,1,0}+N_{1,1,1}+N_{3,1,0}+N_{3,1,1}+N_{5,1,0}+N_{5,1,1}=0 \bmod 2 \text {, } \\
& N_{1,0,1}+N_{1,1,1}+N_{3,0,1}+N_{3,1,1}+N_{5,0,1}+N_{5,1,1}=0 \bmod 2 \text {. }
\end{aligned}
$$

These conditions are equivalent to the rules

$$
\begin{aligned}
& \mathrm{SU}(3): \quad \quad \sum_{l} k^{(l)} n_{3}^{(l)}=0 \bmod 3, \\
& \text { SO(4): } \quad \sum_{l} k^{(l)} n_{2}^{(l)}=0 \bmod 2 \text {, } \\
& \sum_{l} k^{(l)} n_{2}^{\prime(l)}=0 \bmod 2,
\end{aligned}
$$

as stated in (128).

\section{A.3 The gamma phases}

\section{A.3.1 The general case}

Consider a sector $\mathcal{H}_{[g]}$, with representative space group element $g=\left(\theta^{k}, m_{a} \mathbf{e}_{a}\right)$. As explained in Section 2.3, physical states are superpositions of states which are localized at the various fixed points $\mathbf{z}_{l} \in \mathbb{C}^{3}$, corresponding to the elements $l \in[g]$,

$$
|\chi\rangle_{[g]}=\sum_{l \in[g]} \chi_{l}\left|\mathbf{z}_{l}\right\rangle
$$

with coefficients $\chi_{l}$. The gamma phases are then defined by the eigenvalues of the action of other elements $h \in S$ on $\mathcal{H}_{[g]}$.

Any element of the conjugacy class $l \in[g]$ arises from the representative $g$ by conjugation with a twist $\left(\theta^{n}, 0\right)$ and a translation $\left(\mathbb{1}, m_{a}^{\prime} \mathbf{e}_{a}\right)$,

$$
l=\left(\mathbb{1}, m_{a}^{\prime} \mathbf{e}_{a}\right)\left(\theta^{n}, 0\right) g\left(\theta^{-n}, 0\right)\left(\mathbb{1},-m_{a}^{\prime} \mathbf{e}_{a}\right) .
$$

For the associated fixed point $\mathbf{z}_{l}$ this means

$$
\left|\mathbf{z}_{l}\right\rangle=\left(\mathbb{1}, m_{a}^{\prime} \mathbf{e}_{a}\right)\left(\theta^{n}, 0\right)\left|\mathbf{z}_{g}\right\rangle,
$$

and a natural ansatz for the superposition (396) is

$$
|\chi\rangle_{[g]}=\underbrace{\left(\sum_{m_{a}^{\prime} \mathbf{e}_{a} \in \Lambda} \chi_{m_{a}^{\prime} \mathbf{e}_{a}}^{\prime}\left(\mathbb{1}, m_{a}^{\prime} \mathbf{e}_{a}\right)\right)}_{X} \underbrace{\left(\sum_{n=0}^{N-1} \chi_{n}\left(\theta^{n}, 0\right)\right)}_{Y}\left|\mathbf{z}_{g}\right\rangle .
$$


The first bracket $X$ sums over infinitely many terms which correspond to infinitely many lattice translations within $\Lambda$. The second bracket $Y$ represents $N$ rotations of the translational part of $g \in[g]$, where $N$ is the order of the orbifold.

The coefficients $\chi_{m_{a}^{\prime} \mathbf{e}_{a}}^{\prime}$ and $\chi_{n}$ in (399) are not fully arbitrary. First, we require compatibility with the group multiplication rule,

$$
\chi_{\left(m_{a}+m_{a}^{\prime}\right) \mathbf{e}_{a}}^{\prime}=\chi_{m_{a} \mathbf{e}_{a}}^{\prime} \chi_{m_{a}^{\prime} \mathbf{e}_{a}}^{\prime}, \quad \chi_{n+n^{\prime}}=\chi_{n} \chi_{n^{\prime}} \quad \chi_{\theta^{k} m_{a} \mathbf{e}_{a}}^{\prime}=\chi_{m_{a} \mathbf{e}_{a}}^{\prime},
$$

where the latter relation follows from the explicit form of the inverse element (20) and should hold for any $k$. The rotation can be expressed on the coefficients $m_{a}^{\prime}$ by use of the Coxeter element $C^{T}$ of the geometry, which is the rotational matrix defined as

$$
\theta \mathbf{e}_{a} \equiv C_{a b}^{T} \mathbf{e}_{b}
$$

The requirements (400) are then fulfilled by the exponential ansatz

$$
\chi_{m_{a}^{\prime} \mathbf{e}_{a}}^{\prime}=e^{2 \pi i x_{a} m_{a}^{\prime}}, \quad \chi_{n}=e^{2 \pi i y n},
$$

where $x_{1}, \ldots, x_{6}$ and $y$ are some coefficients, constrained by

$$
x_{a}\left(\delta_{a b}-C_{a b}^{-1}\right)=0 \bmod 1, \quad N y=0 \bmod 1 .
$$

Second, elements $h^{\prime}=\left(\theta^{n}, m_{a}^{\prime} \mathbf{e}_{a}\right)$ which do commute with the representative $g=$ $\left(\theta^{k}, m_{a} \mathbf{e}_{a}\right)$ do not affect the state $\left|\mathbf{z}_{g}\right\rangle$, and hence should not appear with different coefficients. The property $\left[g, h^{\prime}\right]=0$ can be expressed as

$$
\left(\mathbb{1}-\theta^{n}\right) m_{a} \mathbf{e}_{a}=\left(\mathbb{1}-\theta^{k}\right) m_{a}^{\prime} \mathbf{e}_{a} .
$$

For the coefficients $m_{a}^{\prime}$ this reads

$$
\left(\delta_{a b}-C_{a b}^{n}\right) m_{b}=\left(\delta_{a b}-C_{a b}^{k}\right) m_{b}^{\prime} .
$$

We now aim to associate the right-hand side of this equation with $x_{a} m_{a}^{\prime}$ in (402), and the left-hand side with yn, with a relative minus sign. However, this requires a linear expression in $n$, up to integers. With a new set of constrained constants $\eta_{a}$ the linearization can be realized:

$$
\eta_{a}\left(\delta_{a b}-C_{a b}^{n}\right)=n \eta_{a}\left(\delta_{a b}-C_{a b}\right) \bmod 1, \quad \text { for all } n \text {. }
$$

This leads to the improved, but still preliminary ansatz

$$
\chi_{m_{a}^{\prime} \mathbf{e}_{a}}^{\prime}=e^{2 \pi i k \gamma_{a}^{\prime} m_{a}^{\prime}}, \quad \chi_{n}=e^{-2 \pi i n \gamma_{a}^{\prime} m_{a}}, \quad \gamma_{a}^{\prime}=\eta_{b}\left(\delta_{b a}-C_{b a}\right),
$$

for which the constraints (403) become

$$
k \eta_{a}\left(\delta_{a b}-C_{a b}\right)\left(\delta_{b c}-C_{b c}^{-1}\right)=0 \bmod 1, \quad \quad N \gamma_{a}^{\prime} m_{a}=0 \bmod 1 .
$$


Note that the two brackets combine to a diagonal matrix, whose entries are given by $2\left(1-\operatorname{Re} \vartheta_{(i)}\right)$, where $\vartheta_{(i)}$ is the twist in the corresponding plane.

So far we have realized the group transformation laws on the coefficients, and we have ensured that elements which commute with the representative element have unit coefficients. They are thus equivalent to the trivial element $(\mathbb{1}, 0)$ within the product of sums $X Y$. However, one can add an additional phase to the terms in $Y$ which is also trivial for commuting elements, without cancelation from the phase in $X$. Let $N_{(g)}$ be the smallest positive integer for which

$$
\left(\mathbb{1}-\theta^{N_{(g)}}\right) m_{a} \mathbf{e}_{a} \in \Lambda_{k}, \quad 1 \leqslant N_{(g)} \leqslant k .
$$

Note that $N_{(g)}$ is a divisor of $N$. By comparison with (404) one finds that for commuting elements $\left(\theta^{n}, m_{a}^{\prime} \mathbf{e}_{a}\right), n$ must be a multiple of $N_{(g)}$. This means that the phase

$$
e^{-2 \pi i \gamma n}, \quad N_{(g)} \gamma=0 \bmod 1,
$$

is one for commuting elements, and we have introduced a new constant $\gamma$. Our final ansatz is then

$$
\chi_{m_{a}^{\prime} \mathbf{e}_{a}}^{\prime}=e^{2 \pi i k \gamma_{a}^{\prime} m_{a}^{\prime}}, \quad \chi_{n}=e^{-2 \pi i n \gamma_{a}^{\prime} m_{a}} e^{-2 \pi i \gamma n},
$$

and one can now study the transformation behavior of the superposition (399), with the suggested coefficients.

As we will show, they fulfill an eigenvalue equation with respect to arbitrary operators $h=\left(\theta^{\tilde{k}}, \tilde{m}_{a} \mathbf{e}_{a}\right) \in S$, of the form

$$
\begin{aligned}
h|\chi\rangle_{[g]}=\left(\theta^{\tilde{k}}, \tilde{m}_{a} \mathbf{e}_{a}\right) X Y\left|\mathbf{z}_{g}\right\rangle & =\left(\mathbb{1}, \tilde{m}_{a} \mathbf{e}_{a}\right)\left(\theta^{\tilde{k}}, 0\right) X Y\left|\mathbf{z}_{g}\right\rangle \\
& =\left(\left(\mathbb{1}, \tilde{m}_{a} \mathbf{e}_{a}\right) X\right)\left(\left(\theta^{\tilde{k}}, 0\right) Y\right)\left|\mathbf{z}_{g}\right\rangle \\
& =\tilde{\lambda}_{\tilde{m}} \lambda_{\tilde{k}}|\chi\rangle_{[g]},
\end{aligned}
$$

where $\tilde{\lambda}_{\tilde{m}}$ and $\lambda_{\tilde{k}}$ are eigenvalues of $X$ and $Y$ under translations and rotations, respectively. This follows the spirit of the separation of space group elements into a translational and a rotational part, which is the crucial feature of the decomposition (399).

On $X$, rotations $(\theta, 0)$ act as

$$
\begin{aligned}
(\theta, 0) X & =\sum_{m_{a}^{\prime} \mathbf{e}_{a} \in \Lambda} e^{2 \pi i k \gamma_{a}^{\prime} m_{a}^{\prime}}(\mathbb{1}, \underbrace{\theta m_{a}^{\prime} \mathbf{e}_{a}}_{m_{a}^{\prime \prime} \mathbf{e}_{a}})(\theta, 0) \\
& =\sum_{m_{a}^{\prime \prime} \mathbf{e}_{a} \in \Lambda} e^{2 \pi i k \gamma_{a}^{\prime} C_{a b}^{-1} m_{b}^{\prime \prime}}\left(\mathbb{1}, m_{a}^{\prime \prime} \mathbf{e}_{a}\right)(\theta, 0)=X(\theta, 0),
\end{aligned}
$$

where the rotational invariance of $k \gamma_{a}^{\prime} m_{a}^{\prime}$ from (403) was used. Thus $X$ commutes with rotations, as a direct consequence of (400). 
Furthermore, $X$ fulfills an eigenvalue equation with respect to translations:

$$
\begin{aligned}
\left(\mathbb{1}, \tilde{m}_{a} \mathbf{e}_{a}\right) X & =\sum_{m_{a}^{\prime} \mathbf{e}_{a} \in \Lambda} e^{2 \pi i k \gamma_{a}^{\prime} m_{a}^{\prime}}(\mathbb{1}, \underbrace{m_{a}^{\prime} \mathbf{e}_{a}+\tilde{m}_{a} \mathbf{e}_{a}}_{m_{a}^{\prime \prime} \mathbf{e}_{a}}) \\
& =e^{-2 \pi i k \gamma_{a}^{\prime} \tilde{m}_{a}} \sum_{m_{a}^{\prime \prime} \mathbf{e}_{a} \in \Lambda} e^{2 \pi i k \gamma_{a}^{\prime} m_{a}^{\prime \prime}}\left(\mathbb{1}, m_{a}^{\prime \prime} \mathbf{e}_{a}\right)=e^{-2 \pi i k \gamma_{a}^{\prime} \tilde{m}_{a}} X .
\end{aligned}
$$

Finally we consider the action of rotations $\theta$ on $Y$ :

$$
\begin{aligned}
(\theta, 0) Y & =\sum_{n=0}^{N-1} e^{-2 \pi i n \gamma_{a}^{\prime} m_{a}} e^{-2 \pi i \gamma n}(\underbrace{\theta^{n+1}}_{\theta^{n^{\prime}}}, 0) \\
& =e^{2 \pi i \gamma_{a}^{\prime} m_{a}} e^{2 \pi i \gamma} \sum_{n^{\prime}=0}^{N} e^{-2 \pi i n^{\prime} \gamma_{a}^{\prime} m_{a}} e^{-2 \pi i \gamma n^{\prime}}\left(\theta^{n^{\prime}}, 0\right)=e^{2 \pi i \gamma_{a}^{\prime} m_{a}} e^{2 \pi i \gamma} Y .
\end{aligned}
$$

In summary, the eigenstates of $h=\left(\theta^{\tilde{k}}, \tilde{m}_{a} \mathbf{e}_{a}\right)$ in the sector $\mathcal{H}_{[g]}, g=\left(\theta^{k}, m_{a} \mathbf{e}_{a}\right)$, are

$$
\begin{aligned}
& \left|\gamma^{\prime}, \gamma\right\rangle_{[g]}=\left(\sum_{m_{a}^{\prime} \mathbf{e}_{a} \in \Lambda} e^{2 \pi i k \gamma_{a}^{\prime} m_{a}^{\prime}}\left(\mathbb{1}, m_{a}^{\prime} \mathbf{e}_{a}\right)\right)\left(\sum_{n=0}^{N-1} e^{-2 \pi i n \gamma_{a}^{\prime} m_{a}} e^{-2 \pi i \gamma n}\left(\theta^{n}, 0\right)\right)\left|\mathbf{z}_{g}\right\rangle, \\
& \left|\gamma^{\prime}, \gamma\right\rangle_{[g]} \stackrel{h}{\mapsto} e^{2 \pi i \gamma_{a}^{\prime}\left(\tilde{k} m_{a}-k \tilde{m}_{a}\right)} e^{2 \pi i \gamma \tilde{k}}\left|\gamma^{\prime}, \gamma\right\rangle_{[g]},
\end{aligned}
$$

where the constants in $\gamma^{\prime}=\left(\gamma_{1}^{\prime}, \ldots, \gamma_{6}^{\prime}\right)$ and $\gamma$ fulfill the constraints

$$
\begin{aligned}
\gamma_{a}^{\prime} & =\eta_{b}\left(\delta_{b a}-C_{b a}\right), & & \\
N_{(g)} \gamma & =0 \bmod 1, & & \\
\eta_{a}\left(\delta_{a u}-C_{a u}^{n}\right) & =n \eta_{a}\left(\delta_{a u}-C_{a u}\right) \bmod 1, & & \text { for all } n, u, \\
2 k\left(1-\operatorname{Re} \vartheta_{\left(i_{u}\right)}\right) \eta_{u} & =0 \bmod 1, & & \text { for all } u,
\end{aligned}
$$

where $\left(i_{1}, \ldots, i_{6}\right)=(1,1,2,2,3,3)$ are the labels of the planes associated with the lattice vectors $\mathbf{e}_{u}$, and $N_{(g)}$ was defined in (409). Note that the condition

$$
N \gamma_{a}^{\prime} m_{a}=0 \bmod 1
$$

is automatically satisfied for solutions of the above constraints. This immediately follows from (418b) with the choice $n=N$.

\section{A.3.2 The $\mathbb{Z}_{6-\text { II }}$ example}

For the $\mathbb{Z}_{6-\text { II }}$ geometry, the transpose $C$ of the Coxeter element from (401) reads

$$
C=\left(\begin{array}{cccccc}
-1 & 1 & & & & \\
-3 & 2 & & & & \\
& & -1 & 1 & & \\
& & -1 & 0 & & \\
& & & & -1 & 0 \\
& & & & 0 & -1
\end{array}\right)
$$


where all entries which are not shown are zero. The conditions (418) for the space group eigenstates (416) for the $\mathbb{Z}_{6-\text { II }}$ geometry are then solved by

$$
\gamma^{\prime}=\left(0,0, \frac{l_{3}}{3}, \frac{l_{3}}{3}, \frac{l_{5}}{2}, \frac{l_{6}}{2}\right),
$$

in terms of integers $l_{3}, l_{5}, l_{6}$. Thus the order of the $a$-th entry of $\gamma^{\prime}$ corresponds to the order of the lattice vector $\mathbf{e}_{a}$, and the two entries for the $\mathrm{SU}(3)$-plane are identical since $\mathbf{e}_{3}$ and $\mathbf{e}_{4}$ are connected by a twist.

\section{B Lie Group Basics}

This section is a very brief review of selected well known facts about Lie groups. Some of them are needed in the main text, especially for the identification of the representations of states, which arise in the effective theories derived from the heterotic string.

\section{Weights}

A Lie group $G$ has an associated Lie algebra $\mathcal{G}$. Its commuting generators form the Cartan sub-algebra $\mathcal{H}=\left\{H_{i}\right\}$ with dimension $r$, dubbed the rank of $G$.

For any representation of dimension $D$, the action of the elements of the Cartan subalgebra $H_{i} \in \mathcal{H}$ can be simultaneously diagonalized. The states then obey a relation

$$
H_{i}|\mu, D\rangle=\mu_{i}|\mu, D\rangle
$$

where the weight vector $\mu=\left(\mu_{1}, \ldots, \mu_{r}\right)$ collects the weights $\mu_{i}$ of the representation $D$. In the main text, the weight vectors are given by the shifted left-moving momenta $\mathbf{p}_{\mathrm{sh}}$ of the generated states.

\section{Roots}

Roots $\alpha_{i}$ are the weights of the adjoint representation. They obey

$$
\left[H_{i}, E_{\alpha}\right]=\alpha_{i} E_{\alpha}, \quad\left[E_{\alpha}, E_{-\alpha}\right]=\alpha \cdot H
$$

and the corresponding states $E_{\alpha}$ act as raising and lowering operators:

$$
\left[E_{\alpha}, E_{\beta}\right] \sim E_{\alpha+\beta}, \quad H_{i} E_{ \pm \alpha}|\mu, D\rangle=\left(\mu_{i} \pm \alpha_{i}\right) E_{ \pm \alpha}|\mu, D\rangle .
$$

For each root eigenstate $E_{\alpha}$, there is a natural $S U(2)$ subgroup generated by

$$
E^{ \pm} \equiv|\alpha|^{-1} E_{ \pm \alpha}, \quad E_{3} \equiv|\alpha|^{-2} \alpha \cdot H
$$

such that the $E^{ \pm}$raise and lower the eigenvalue of $E_{3}$ of a state, respectively. In general,

$$
E_{3}|\mu, D\rangle=\frac{\alpha \cdot \mu}{\alpha^{2}}|\mu, D\rangle
$$


and $\alpha \cdot \mu / \alpha^{2}$ in any representation is an integer or half integer. As always for representations of $S U(2)$ there are a non-negative integers $p, q$ such that

$$
\begin{array}{rlrl}
\left(E^{+}\right)^{p}|\mu, D\rangle \neq 0, & & \left(E^{+}\right)^{p+1}|\mu, D\rangle=0 \\
\left(E^{-}\right)^{q}|\mu, D\rangle \neq 0, & \left(E^{-}\right)^{q+1}|\mu, D\rangle=0
\end{array}
$$

In that case, the highest and lowest weights are $(\mu+p \alpha)$ and $(\mu-q \alpha)$, respectively, and

$$
\begin{aligned}
& E_{3}|\mu+p \alpha, D\rangle=\left(\frac{\alpha \cdot \mu}{\alpha^{2}}+p\right)|\mu+p \alpha, D\rangle \equiv j|\mu+p \alpha, D\rangle, \\
& E_{3}|\mu-q \alpha, D\rangle=\left(\frac{\alpha \cdot \mu}{\alpha^{2}}-q\right)|\mu-q \alpha, D\rangle \equiv-j|\mu-q \alpha, D\rangle,
\end{aligned}
$$

where $j$ is the spin of the representation. One then finds the formula

$$
\frac{\alpha \cdot \mu}{\alpha^{2}}=-\frac{1}{2}(p-q) \text {. }
$$

The right hand side gives a label to the state with weight $\mu$, compare e.g. the $j=3 / 2$ representation, where $(p-q) / 2 \in\{-3 / 2,-1 / 2,1 / 2,3 / 2\}$. The left hand side, when applied to the adjoint representation, gives the cosine of the angle between roots. The integer nature of $p, q$ then restricts the possible angles to $90,120,135$ or 150 degrees. These are the angles appearing in Dynkin- and root diagrams.

\section{Simple Roots}

A root is said to be positive, if the first non-zero entry in some fixed basis is positive. It is negative, if the first non-zero entry is negative. With this definition in the adjoint representation the positive roots correspond to raising, the negative to lowering operators. For roots $\mu, \nu$ we define

$$
\mu>\nu \quad \text { if }(\mu-\nu) \text { is positive. }
$$

Simple roots are positive roots that cannot be written as a sum of other positive roots. The number of simple roots equals the rank $r$ of the group. All other roots and thus the whole algebra can be deduced from linear combinations of simple roots.

\section{Cartan Matrix}

The Cartan matrix is defined as

$$
A_{i j} \equiv \frac{2}{\alpha_{i}^{2}}\left(\alpha_{i} \cdot \alpha_{j}\right)
$$

For fixed $j$, its entries give the $(p-q)$ values of the $S U(2)_{i}$ 's associated with the simple roots $\alpha_{i}$, when acting on a the state $E_{\alpha_{j}}$. The Cartan matrix is characteristic for a group, it contains the same information as the associated Dynkin diagram. Non-zero off-diagonal entries, or equivalently non-zero angles between roots, describe a non-trivial overlap of the contained $\mathrm{SU}(2)_{i}$ subgroups, generated by the simple roots $E_{\alpha_{i}}$. 


\section{Dynkin Labels and Fundamental Weights}

In the spirit of (429) one can define the 'Cartan-Weyl labels' $l_{i}$ for any state $|\mu, D\rangle$ of a irreducible representation $D$ (here no summation over $i$ )

$$
l_{i} \equiv \frac{2}{\alpha_{i}^{2}}\left(\alpha_{i} \cdot \mu\right)=-\frac{1}{2}\left(p_{i}-q_{i}\right), \quad i=1, \ldots, r .
$$

The integers $p_{i}, q_{i}$ specify how often the simple root $E_{\alpha_{i}}$ can be applied before the state reaches zero, cf. (427). Now consider the application of $E_{ \pm \alpha_{j}}$ to the state $|\mu, D\rangle$. It results in $\mu \mapsto \mu \pm \alpha_{j}$, and

$$
l_{i} \stackrel{E_{ \pm \alpha_{j}}}{\longmapsto} l_{i} \pm \frac{2}{\alpha_{i}^{2}}\left(\alpha_{i} \cdot \alpha_{j}\right)=l_{i} \pm A_{i j}
$$

The difference of the vectors $l=\left(l_{1}, \ldots, l_{r}\right)$ for two different states of a representation is thus always given by one of the columns of the Cartan matrix. This is the property which is used in the main text in order to identify the weights $\mu=\mathbf{p}_{\mathrm{sh}}$ that correspond to the same representation $D$.

For any irreducible representation $D$ there is a maximal weight $\mu$ such that

$$
E_{\alpha_{i}}|\mu, D\rangle=0 \quad \forall i
$$

Thus for this weight one has $p_{i}=0$ for all $i$, and the set of integers

$$
\left(l_{1}, \ldots, l_{r}\right)
$$

fully determines the representation. These labels $l_{i}$ are called Dynkin labels of the representation $D$.

Furthermore, one defines fundamental weights $\mu^{i}$,

$$
\frac{2}{\alpha_{i}^{2}}\left(\alpha_{i} \ldots \mu^{j}\right)=\delta_{i}^{j},
$$

which imply the decomposition of general weights as

$$
\mu=\sum_{i=1}^{r} l_{i} \mu^{i} .
$$

This gives another interpretation for the quantities $l_{i}$ from (431). In (436) they play the role of coefficients of the weight vector with respect to a basis in weight space, spanned by the fundamental weights $\mu^{j}$. 


\section{Anomalies with Abelian factors}

\section{C.1 Bulk anomalies}

The contributions to the bulk anomaly polynomial $I_{8}$ which involve Abelian field strength two-forms $\hat{F}_{u}, u=1, \ldots, 5$, canonically normalized as in (219), take the form (218),

$$
\begin{aligned}
I_{(1 / 2)}^{\text {Abelian }}\left(F^{4}, R^{0}\right)= & \frac{8}{3} \sum_{A} \sum_{u=1}^{5} a_{A}^{u}\left(\operatorname{tr} F_{A}^{3}\right) \hat{F}_{u}+4 \sum_{A} \sum_{u, v=1}^{5} b_{A}^{u v}\left(\operatorname{tr} F_{A}^{2}\right) \hat{F}_{u} \hat{F}_{v} \\
& +\frac{2}{3} \sum_{u, v, w, x=1}^{5} c^{u v w z} \hat{F}_{u} \hat{F}_{v} \hat{F}_{w} \hat{F}_{x} \\
I_{(1 / 2)}^{\text {Abelian }}\left(F^{2}, R^{2}\right)= & -\frac{1}{6}\left(\operatorname{tr} R^{2}\right) \sum_{u, v=1}^{5} d^{u v} \hat{F}_{u} \hat{F}_{v},
\end{aligned}
$$

where $A=\mathrm{SU}(6), \mathrm{SU}(3), \mathrm{SO}(8)$ labels the non-Abelian gauge groups in the bulk. The different relative multiplicities arise from the various choices of associating the non-Abelian groups with some of the field strength two-forms, and the Abelian group factors with the others.

The coefficients $a_{A}^{u}, b_{A}^{u v}, c^{u v w x}, d^{u v}$ follow from summations over all hyperinos $\tilde{h}$ in the bulk. Each of them transforms in a representation of the gauge group and has charges under the U(1)'s,

$$
\tilde{h}:\left\{\begin{array}{l}
\text { representation } \mathcal{R}_{A}(\tilde{h}) \text { w.r.t. group } A \\
\text { charge } Q_{u}(\tilde{h}) \text { w.r.t. } U(1)_{u} .
\end{array}\right.
$$

For the conversion of this information into quantities of relevance for (437) we use the identities from Table 13 and the definition (219),

$$
\operatorname{tr}_{\mathcal{R}} F_{A}^{2} \equiv v_{A}^{\mathcal{R}} \operatorname{tr} F_{A}^{2}, \quad \operatorname{tr}_{\mathcal{R}} F_{A}^{3} \equiv w_{A}^{\mathcal{R}} \operatorname{tr} F_{A}^{3}, \quad Q_{u}=\sqrt{2}\left|t_{u}\right| \hat{Q}_{u} .
$$

The hyperino charge sums then read

$$
\begin{aligned}
a_{A}^{u} & =\sum_{\tilde{h}} w_{A}^{\mathcal{R}(\tilde{h})} \hat{Q}_{u}(\tilde{h}), \\
b_{A}^{u v} & =\sum_{\tilde{h}} v_{A}^{\mathcal{R}(\tilde{h})} \hat{Q}_{u}(\tilde{h}) \hat{Q}_{v}(\tilde{h}), \\
c^{u v w x} & =\sum_{\tilde{h}} \hat{Q}_{u}(\tilde{h}) \hat{Q}_{v}(\tilde{h}) \hat{Q}_{w}(\tilde{h}) \hat{Q}_{x}(\tilde{h}), \\
d^{u v} & =\sum_{\tilde{h}} \hat{Q}_{u}(\tilde{h}) \hat{Q}_{v}(\tilde{h}) .
\end{aligned}
$$




\begin{tabular}{|c|c|c|c|c|c|c|c|c|}
\hline \multicolumn{2}{|c|}{$\mathrm{SU}(3)$} & \multicolumn{2}{|c|}{$\mathrm{SU}(4)$} & \multicolumn{2}{|c|}{$\mathrm{SU}(5)$} & \multicolumn{2}{|c|}{$\mathrm{SU}(6)$} & $\mathrm{SO}(8)$ \\
\hline$v^{3}=1$ & $w^{3}=1$ & $v^{4}=1$ & $w^{4}=1$ & $v^{\mathbf{5}}=1$ & $w^{5}=1$ & $v^{\mathbf{6}}=1$ & $w^{6}=1$ & $v^{8}=1$ \\
\hline$v^{\overline{3}}=1$ & $w^{\overline{3}}=-1$ & $v^{\overline{4}}=1$ & $w^{\overline{4}}=-1$ & $v^{\overline{5}}=1$ & $w^{\overline{5}}=-1$ & $v^{\overline{\mathbf{6}}}=1$ & $w^{\overline{\mathbf{6}}}=-1$ & $v^{\boldsymbol{8}_{s}}=1$ \\
\hline & & $v^{6}=2$ & $w^{6}=0$ & $v^{10}=3$ & $w^{\mathbf{1 0}}=1$ & $v^{20}=6$ & $w^{20}=0$ & $v^{\boldsymbol{8}_{c}}=1$ \\
\hline & & & & $v^{\overline{10}}=3$ & $w^{\overline{10}}=-1$ & & & \\
\hline
\end{tabular}

Table 24: The constants $v_{A}^{\mathcal{R}}$ and $w_{A}^{\mathcal{R}}$ for a choice of gauge groups $A$ and representations $\mathcal{R}$.

Note that $\mathrm{SO}(8)$ has no third order invariant.

We now evaluate these sums for the bulk hypermultiplets listed in the Tables 6 and 7 . With $w_{A}^{\mathcal{R}}$ from Table 24 and the given charges follows immediately that

$$
a_{\mathrm{SU}(6)}^{u}=a_{\mathrm{SU}(3)}^{u}=a_{\mathrm{SO}(8)}^{u}=0, \quad \text { for all } u=1, \ldots, 5 .
$$

Furthermore, we find

$$
b_{\mathrm{SU}(6)}^{u v}=b_{\mathrm{SU}(3)}^{u v}=2 b_{\mathrm{SO}(8)}^{u v}=\frac{1}{2} \beta^{u v}, \quad \quad d^{u v}=6\left(\beta^{u v}+\delta^{u v}\right),
$$

with a symmetric matrix $\beta$,

$$
\left(\beta^{u v}\right)=\left(\begin{array}{ccccc}
3 & -1 & 0 & -1 & 0 \\
-1 & 3 & 0 & -1 & 0 \\
0 & 0 & 2 & 0 & \sqrt{2} \\
-1 & -1 & 0 & 4 & 0 \\
0 & 0 & \sqrt{2} & 0 & 4
\end{array}\right)
$$

The coefficients $c^{u v w x}$ are listed in Table 25. Close inspection shows that they can be written as

$$
c^{u v w x}=\frac{3}{2|\sigma(u, v, w, x)|}\left(\delta^{u v} \beta^{w x}+\text { permutations }\right) .
$$

Here $|\sigma(u, v, w, x)|$ is a symmetry factor, which is only different from one if two or more of its variables are equal. In that case it counts the possibilities to associate these equal labels with equally many of the four $F$-factors. For example,

$$
|\sigma(3,4,4,5)|=\left(\begin{array}{l}
4 \\
2
\end{array}\right)=6 \quad \Rightarrow \quad c^{3445}=\frac{3}{2 \cdot 6}\left(\delta^{44} \beta^{35}+0\right)=\frac{1}{2 \sqrt{2}} .
$$

\section{C.2 Fixed point anomalies}

The anomaly polynomial at the fixed points characterized by $n_{2}=0,1$ is a six-form of the form

$$
I_{6}=\operatorname{tr} R^{2} \sum_{v} \tilde{a}^{v} \hat{F}_{v}-8 \sum_{B} \tilde{b}_{B} \operatorname{tr} F_{B}^{3}-24 \sum_{B, v} \tilde{c}_{B}^{v} \operatorname{tr} F_{B}^{2} \hat{F}_{v}-8 \sum_{v, w, x} \tilde{d}_{B}^{v w x} \hat{F}_{v} \hat{F}_{w} \hat{F}_{x},
$$




\begin{tabular}{c|c|c|c|c||c|c|c|c|c||c|c|c|c|c||c|c|c|c|c}
$u$ & $v$ & $w$ & $x$ & $h$ & $u$ & $v$ & $w$ & $x$ & $h$ & $u$ & $v$ & $w$ & $x$ & $h$ & $u$ & $v$ & $w$ & $x$ & $h$ \\
\hline \hline 1 & 1 & 1 & 1 & $\frac{9}{2}$ & 1 & 2 & 2 & 5 & 0 & 2 & 2 & 2 & 3 & 0 & 2 & 5 & 5 & 5 & 0 \\
1 & 1 & 1 & 2 & $-\frac{3}{4}$ & 1 & 2 & 3 & 3 & $-\frac{1}{4}$ & 2 & 2 & 2 & 4 & $-\frac{3}{4}$ & 3 & 3 & 3 & 3 & 3 \\
1 & 1 & 1 & 3 & 0 & 1 & 2 & 3 & 4 & 0 & 2 & 2 & 2 & 5 & 0 & 3 & 3 & 3 & 4 & 0 \\
1 & 1 & 1 & 4 & $-\frac{3}{4}$ & 1 & 2 & 3 & 5 & 0 & 2 & 2 & 3 & 3 & $\frac{5}{4}$ & 3 & 3 & 3 & 5 & $\frac{3}{2 \sqrt{2}}$ \\
1 & 1 & 1 & 5 & 0 & 1 & 2 & 4 & 4 & $-\frac{1}{4}$ & 2 & 2 & 3 & 4 & 0 & 3 & 3 & 4 & 4 & $\frac{3}{2}$ \\
1 & 1 & 2 & 2 & $\frac{3}{2}$ & 1 & 2 & 4 & 5 & 0 & 2 & 2 & 3 & 5 & $\frac{1}{2 \sqrt{2}}$ & 3 & 3 & 4 & 5 & 0 \\
1 & 1 & 2 & 3 & 0 & 1 & 2 & 5 & 5 & $-\frac{1}{4}$ & 2 & 2 & 4 & 4 & $\frac{7}{4}$ & 3 & 3 & 5 & 5 & $\frac{3}{2}$ \\
1 & 1 & 2 & 4 & $-\frac{1}{4}$ & 1 & 3 & 3 & 3 & 0 & 2 & 2 & 4 & 5 & 0 & 3 & 4 & 4 & 4 & 0 \\
1 & 1 & 2 & 5 & 0 & 1 & 3 & 3 & 4 & $-\frac{1}{4}$ & 2 & 2 & 5 & 5 & $\frac{7}{4}$ & 3 & 4 & 4 & 5 & $\frac{1}{2 \sqrt{2}}$ \\
1 & 1 & 3 & 3 & $\frac{5}{4}$ & 1 & 3 & 3 & 5 & 0 & 2 & 3 & 3 & 3 & 0 & 3 & 4 & 5 & 5 & 0 \\
1 & 1 & 3 & 4 & 0 & 1 & 3 & 4 & 4 & 0 & 2 & 3 & 3 & 4 & $-\frac{1}{4}$ & 3 & 5 & 5 & 5 & 3 \\
1 & 1 & 3 & 5 & $\frac{1}{2 \sqrt{2}}$ & 1 & 3 & 4 & 5 & 0 & 2 & 3 & 3 & 5 & 0 & 4 & 4 & 4 & 4 & 6 \\
1 & 1 & 4 & 4 & $\frac{7}{4}$ & 1 & 3 & 5 & 5 & 0 & 2 & 3 & 4 & 4 & 0 & 4 & 4 & 4 & 5 & 0 \\
1 & 1 & 4 & 5 & 0 & 1 & 4 & 4 & 4 & $-\frac{3}{4}$ & 2 & 3 & 4 & 5 & 0 & 4 & 4 & 5 & 5 & 2 \\
1 & 1 & 5 & 5 & $\frac{7}{4}$ & 1 & 4 & 4 & 5 & 0 & 2 & 3 & 5 & 5 & 0 & 4 & 5 & 5 & 5 & 0 \\
1 & 2 & 2 & 2 & $-\frac{3}{4}$ & 1 & 4 & 5 & 5 & $-\frac{1}{4}$ & 2 & 4 & 4 & 4 & $-\frac{3}{4}$ & 5 & 5 & 5 & 5 & 6 \\
1 & 2 & 2 & 3 & 0 & 1 & 5 & 5 & 5 & 0 & 2 & 4 & 4 & 5 & 0 & & & & & \\
1 & 2 & 2 & 4 & $-\frac{1}{4}$ & 2 & 2 & 2 & 2 & $\frac{9}{2}$ & 2 & 4 & 5 & 5 & $-\frac{1}{4}$ & & & & &
\end{tabular}

Table 25: The totally symmetric coefficients $c^{u v w x}$.

where $\tilde{a}^{v}, \tilde{b}_{B}, \tilde{c}_{B}^{v}, d^{v w x}$ are coefficients, and $B$ and $v, w, x$ label the local non-Abelian and Abelian gauge group factors, respectively, cf. (225). The canonically normalized U(1) factors $\hat{F}_{v}$ were defined in (219).

The coefficients are calculated by summing over the chiral fermions which are present at the fixed point. They can either arise from twisted sectors, in which case we denote them as $\psi_{l}$, or they are contained in a bulk hypermultiplet, denoted as $\psi_{b}$. As detailed calculations show, see for example [79], the contributions of the latter are weighted with a relative factor of $\frac{1}{4}$ compared to the localized fermions. This is interpreted as a democratic distribution of their effect among the four fixed points of the geometry. 
The coefficients in (446) are given by the sums

$$
\begin{aligned}
\tilde{a}^{v} & =\sum_{\psi_{l}} \hat{Q}_{v}\left(\psi_{l}\right)+\frac{1}{4} \sum_{\psi_{b}} \hat{Q}_{v}\left(\psi_{b}\right) \\
\tilde{b}_{B} & =\sum_{\psi_{l}} w_{B}^{\mathcal{R}\left(\psi_{l}\right)}+\frac{1}{4} \sum_{\psi_{b}} w_{B}^{\mathcal{R}\left(\psi_{b}\right)} \\
\tilde{c}_{B}^{v} & =\sum_{\psi_{l}} v_{B}^{\mathcal{R}\left(\psi_{l}\right)} \hat{Q}_{v}\left(\psi_{l}\right)+\frac{1}{4} \sum_{\psi_{b}} v_{B}^{\mathcal{R}\left(\psi_{b}\right)} \hat{Q}_{v}\left(\psi_{b}\right), \\
\tilde{d}^{v w x} & =\sum_{\psi_{l}} \hat{Q}_{v}\left(\psi_{l}\right) \hat{Q}_{w}\left(\psi_{l}\right) \hat{Q}_{x}\left(\psi_{l}\right)+\frac{1}{4} \sum_{\psi_{b}} \hat{Q}_{v}\left(\psi_{b}\right) \hat{Q}_{w}\left(\psi_{b}\right) \hat{Q}_{x}\left(\psi_{b}\right),
\end{aligned}
$$

where $v_{B}^{\mathcal{R}}$ and $w_{B}^{\mathcal{R}}$ were defined in $(439)$.

With Tables 8, 10, 12, 11 and 26, 27, 28, 29, 24 we evaluate these sums and find

$$
\begin{aligned}
& n_{2}=0: \quad \tilde{a}=(0,-4 \sqrt{2}, 0,5 \sqrt{2},-\sqrt{6}, 2 \sqrt{15}), \quad \tilde{b}=(0,0,0), \\
& \tilde{c}_{\mathrm{SU}(5)}=\tilde{c}_{\mathrm{SU}(3)}=2 \tilde{c}_{\mathrm{SO}(8)}=\left(0,-\frac{\sqrt{2}}{3}, 0, \frac{5}{6 \sqrt{2}},-\frac{1}{2 \sqrt{6}}, \frac{1}{2} \sqrt{\frac{5}{3}}\right), \\
& n_{2}=1: \quad \tilde{a}=\left(\frac{19}{8 \sqrt{2}},-\frac{19}{24 \sqrt{2}}, \frac{19}{8 \sqrt{3}},-\frac{19}{6 \sqrt{2}}, \frac{19}{2 \sqrt{6}}, 0,0,0\right), \quad \tilde{b}=(0,0,0,0), \\
& \tilde{c}_{\mathrm{SU}(2)}=\tilde{c}_{\mathrm{SU}(4)}=\tilde{c}_{\mathrm{SU}(2)^{\prime}}=\tilde{c}_{\mathrm{SU}(4)^{\prime}} \\
& =\left(\frac{1}{4 \sqrt{2}},-\frac{1}{12 \sqrt{2}}, \frac{1}{4 \sqrt{3}},-\frac{1}{3 \sqrt{2}}, \frac{1}{\sqrt{6}}, 0,0,0\right) \text {. }
\end{aligned}
$$

We can bring this into a much simpler form by changing the basis of the Abelian generators. With

$$
\begin{array}{ll}
t_{\mathrm{an}}^{0} \equiv-4 t_{2}+5 t_{4}-t_{5}+t_{6}^{0}, & \hat{t}_{\mathrm{an}}^{0}=\frac{t_{\mathrm{an}}^{0}}{\sqrt{2}\left|t_{\mathrm{an}}^{0}\right|}, \\
t_{\mathrm{an}}^{1} \equiv 3 t_{1}-t_{2}+t_{3}-4 t_{4}+4 t_{5}, & \hat{t}_{\mathrm{an}}^{1}=\frac{t_{\mathrm{an}}^{1}}{\sqrt{2}\left|t_{\mathrm{an}}^{1}\right|},
\end{array}
$$

and orthogonal sets of generators which extend $\hat{t}_{\text {an }}^{0}$ and $\hat{t}_{\text {an }}^{1}$ to a basis at $n_{2}=0$ or $n_{2}=1$, 
respectively, the above results become

$$
\begin{aligned}
n_{2}=0: \quad \sum_{v} \tilde{a}^{v} \hat{F}_{v} & =2 \sqrt{37} \hat{F}_{\text {an }}^{0}, \quad \tilde{b}=(0,0,0), \\
\sum_{v} \tilde{c}_{\mathrm{SU}(5)}^{v} \hat{F}_{v} & =\sum_{v} \tilde{c}_{\mathrm{SU}(3)}^{v} \hat{F}_{v}=2 \sum_{v} \tilde{c}_{\mathrm{SO}(8)}^{v} \hat{F}_{v}=\frac{1}{6} \sqrt{37} \hat{F}_{\mathrm{an}}^{0}, \\
\sum_{2}=1: \quad \tilde{a}^{v} \hat{F}_{v} & =2 \sqrt{10} \hat{F}_{\mathrm{an}}^{1}, \quad \tilde{b}=(0,0,0,0), \\
\sum_{v} \tilde{c}_{\mathrm{SU}(2)}^{v} \hat{F}_{v} & =\sum_{v} \tilde{c}_{\mathrm{SU}(4)}^{v} \hat{F}_{v}=\sum_{v} \tilde{c}_{\mathrm{SU}(2)^{\prime}}^{v} \hat{F}_{v}=\sum_{v} \tilde{c}_{\mathrm{SU}(4)^{\prime}} \hat{F}_{v} \\
& =\frac{1}{6} \sqrt{10} \hat{F}_{\mathrm{an}}^{1} .
\end{aligned}
$$

Note that the appearing square roots are a consequence of

$$
\sqrt{2}\left|t_{\mathrm{an}}^{0}\right|=2 \sqrt{37}, \quad \sqrt{2}\left|t_{\mathrm{an}}^{1}\right|=4 \sqrt{10} .
$$

Finally, we calculate the sums $d^{v w x}$ :

$$
\begin{array}{ll}
n_{2}=0: & \sum_{v w x} d^{v w x} \hat{F}_{v} \hat{F}_{w} \hat{F}_{x}=\frac{1}{8}\left(\sum_{u v} \hat{F}_{u} \frac{\hat{t}_{u} \cdot \hat{t}_{v}}{\left|\hat{t}_{u}\right|\left|\hat{t}_{v}\right|} \hat{F}_{v}\right) 2 \sqrt{37} \hat{F}_{\text {an }}^{0}, \\
n_{2}=1: & \sum_{v w x} d^{v w x} \hat{F}_{v} \hat{F}_{w} \hat{F}_{x}=\frac{1}{8}\left(\sum_{u v} \hat{F}_{u} \frac{\hat{t}_{u} \cdot \hat{t}_{v}}{\left|\hat{t}_{u}\right|\left|\hat{t}_{v}\right|} \hat{F}_{v}\right) 2 \sqrt{10} \hat{F}_{\text {an }}^{1} .
\end{array}
$$

For an orthogonal basis, the brackets sum to $\sum_{u} \hat{F}_{u} \hat{F}_{u}$.

\section{The hidden brane}

The effective orbifold GUT in six dimensions which is described in Chapter 3 has two pairs of inequivalent fixed points. They differ in the localization label $n_{2}$ and their role in the compactification:

$$
\begin{array}{ll}
n_{2}=0: & \text { Physical brane, with local SU(5) GUT, } \\
n_{2}=1: & \text { Hidden brane with exotics, breaks GUT group. }
\end{array}
$$

The hidden brane fixed point is responsible for the breaking of the GUT gauge group to the standard model gauge group in the effective theory in four dimensions.

\section{Localized states at $n_{2}=1$}

We now calculate the matter spectrum at $n_{2}=0$. This requires to solve the mass equations (79), (80), and simultaneously the projection conditions (134a). Consider a sector $\mathcal{H}_{\left[g_{f}\right]}$ 
which gives rise to localized states at $\left(n_{2}, n_{2}^{\prime}\right)=(1,0)$. The case $\left(n_{2}, n_{2}^{\prime}\right)=(1,1)$ will be completely analogous. The elements $g_{f}$ are then further characterized by

$$
\begin{array}{r}
g_{f}: \quad \quad \quad \quad \mathbf{n}: \begin{cases}n_{6}=0, \quad n_{3}=0,1,2, & \text { if } k=1,5 \\
n_{6}=0,1, \quad n_{3}=0, & \text { if } k=3 .\end{cases} \\
\gamma_{a}^{\prime} m_{a}^{(g)}=\frac{l_{3}}{3} n_{3}, \quad \gamma=\frac{j}{3},
\end{array}
$$

where

$$
l_{3}=\left\{\begin{array}{ll}
1,2,3, & \text { if } k=1,5, \\
0, & \text { if } k=3,
\end{array}, \quad j= \begin{cases}0, & \text { if } k=1,5 \\
0,1,2,3, & \text { if } k=3\end{cases}\right.
$$

By the arguments of Section 3.2.3 we find projection conditions

$$
\begin{aligned}
\mathbf{p}_{\mathrm{sh}} \cdot \mathbf{V}_{g_{f}}-\mathbf{R} \cdot \mathbf{v}_{g_{f}} & =0 \bmod 1 \\
\mathbf{p}_{\mathrm{sh}} \cdot\left(\mathbf{V}_{6}+n_{3} \mathbf{W}_{(3)}+\mathbf{W}_{(2)}\right)-\mathbf{R} \cdot \mathbf{v}_{6}+\frac{j}{3} & =0 \bmod 1 \\
\mathbf{p}_{\mathrm{sh}} \cdot \mathbf{W}_{(3)}-\frac{l_{3} k}{3} & =0 \bmod 1 .
\end{aligned}
$$

These equations have 96 solutions which can be casted into multiplets of the local gauge symmetry $\mathrm{SU}(2) \times \mathrm{SU}(4) \times\left[\mathrm{SU}(2)^{\prime} \times \mathrm{SU}(4)^{\prime}\right]$. The result is given in Table 26 . All states are exotics and have to be decoupled from the low-energy spectrum. An example for this is given in [13].

The chiral projection of bulk hypermultiplets to the fixed points $n_{2}=1$ is summarized in Tables 27, 29.

\section{Projection of bulk states}

The local projection condition for bulk fields to fixed points $z_{g_{f}}^{3}$ with $n_{2}=1$ is

\begin{tabular}{|c|c|c|c|c|c|c|c|c|c|c|c|c|c|c|}
\hline Bulk & \multicolumn{2}{|l|}{$n_{2}=1$} & $n_{3}$ & $\gamma_{3}^{\prime}$ & $H_{L}$ & $H_{R}$ & $t_{1}$ & $t_{2}$ & $t_{3}$ & $t_{4}$ & $t_{5}$ & $t_{6}^{1}$ & $t_{7}$ & $t_{8}$ \\
\hline \multirow[t]{2}{*}{$(6, \mathbb{1}, \mathbb{1})$} & $(2, \mathbb{1}, \mathbb{1}, \mathbb{1})$ & & 0 & 1 &,,+-+ &,,-+- & 0 & $-\frac{1}{3}$ & -1 & $\frac{2}{3}$ & 0 & 10 & 0 & 0 \\
\hline & $(\mathbb{1}, \mathbf{4}, \mathbb{1}, \mathbb{1})$ & & 0 & $\frac{1}{3}$ &,,-+- &,,+-+ & 0 & $-\frac{1}{3}$ & 1 & $\frac{2}{3}$ & 0 & 5 & 0 & 0 \\
\hline \multirow[t]{2}{*}{$(\overline{\mathbf{6}}, \mathbb{1}, \mathbb{1})$} & $(2, \mathbb{1}, \mathbb{1}, \mathbb{1})$ & & 0 & $\frac{1}{3}$ &,,+-+ &,,-+- & 0 & $-\frac{1}{3}$ & 1 & $\frac{2}{3}$ & 0 & -10 & 0 & 0 \\
\hline & $(\mathbb{1}, \overline{4}, \mathbb{1}, \mathbb{1})$ & & 0 & 1 &,,-+- &,,+-+ & 0 & $-\frac{1}{3}$ & -1 & $\frac{2}{3}$ & 0 & -5 & 0 & 0 \\
\hline$(\mathbb{1}, \mathbb{1}, \mathbb{1})$ & $(\mathbb{1}, \mathbb{1}, \mathbb{1}, \mathbb{1})$ & $Y_{0}$ & 0 & $\frac{2}{3}$ &,,+-+ & $-1,+\sigma^{2}-$ & 1 & $-\frac{1}{3}$ & 0 & $\frac{2}{3}$ & 0 & 0 & 0 & 0 \\
\hline$(\mathbb{1}, \mathbb{1}, \mathbb{1})$ & $(\mathbb{1}, \mathbb{1}, \mathbb{1}, \mathbb{1})$ & $\bar{Y}_{0}$ & 0 & $\frac{2}{3}$ &,,+-+ & $\begin{array}{l}110 \\
-,+,-\end{array}$ & -1 & $-\frac{1}{3}$ & 0 & $\frac{2}{3}$ & 0 & 0 & 0 & 0 \\
\hline
\end{tabular}

$$
\begin{aligned}
P_{f}: \quad \phi\left(z_{g_{f}}^{3}+z^{3}\right) & =\eta_{f}(\phi) \phi\left(z_{g_{f}}^{3}-z^{3}\right), \\
\eta_{f}(\phi) & =e^{2 \pi i\left(\mathbf{p}_{\mathrm{sh}} \cdot\left(\mathbf{V}_{6}+\mathbf{W}_{(2)}\right)-\mathbf{R} \cdot \mathbf{v}_{6}+\frac{j}{2}\right)}
\end{aligned}
$$

where $j=0$ is understood for fields from the untwisted sector. The results are listed in Tables 27, 28 and 29. 
Table 29 - continued from previous page

\begin{tabular}{|c|c|c|c|c|c|c|c|c|c|c|c|c|c|c|}
\hline Bulk & \multicolumn{2}{|l|}{$n_{2}=1$} & $n_{3}$ & $\gamma_{3}^{\prime}$ & $H_{L}$ & $H_{R}$ & $t_{1}$ & $t_{2}$ & $t_{3}$ & $t_{4}$ & $t_{5}$ & $t_{6}^{1}$ & $t_{7}$ & $t_{8}$ \\
\hline \multirow[t]{2}{*}{$(\mathbb{1}, \mathbf{3}, \mathbb{1})$} & $(\mathbb{1}, \mathbb{1}, \mathbf{2}, \mathbb{1})$ & & 0 & $\frac{1}{3}$ &,,+-+ &,,-+- & 0 & $\frac{2}{3}$ & 0 & $-\frac{1}{3}$ & 1 & 0 & 1 & 0 \\
\hline & $(\mathbb{1}, \mathbb{1}, \mathbb{1}, \mathbb{1})$ & & 0 & $\frac{1}{3}$ &,,-+- &,,+-+ & 0 & $\frac{2}{3}$ & 0 & $-\frac{1}{3}$ & 1 & 0 & -2 & 0 \\
\hline \multirow[t]{2}{*}{$(\mathbb{1}, \overline{\mathbf{3}}, \mathbb{1})$} & $(\mathbb{1}, \mathbb{1}, \mathbf{2}, \mathbb{1})$ & & 0 & 1 &,,-+- &,,+-+ & 0 & $\frac{2}{3}$ & 0 & $-\frac{1}{3}$ & -1 & 0 & -1 & 0 \\
\hline & $(\mathbb{1}, \mathbb{1}, \mathbb{1}, \mathbb{1})$ & & 0 & 1 &,,+-+ &,,-+- & 0 & $\frac{2}{3}$ & 0 & $-\frac{1}{3}$ & -1 & 0 & 2 & 0 \\
\hline \multirow[t]{2}{*}{$(\mathbb{1}, \mathbb{1}, \mathbf{8})$} & $(\mathbb{1}, \mathbb{1}, \mathbb{1}, \mathbf{4})$ & & 0 & $\frac{2}{3}$ &,,+-+ &,,-+- & 0 & $\frac{2}{3}$ & 0 & $-\frac{1}{3}$ & 0 & 0 & 0 & -1 \\
\hline & $(\mathbb{1}, \mathbb{1}, \mathbb{1}, \overline{\mathbf{4}})$ & & 0 & $\frac{2}{3}$ &,,-+- &,,+-+ & 0 & $\frac{2}{3}$ & 0 & $-\frac{1}{3}$ & 0 & 0 & 0 & 1 \\
\hline \multirow[t]{2}{*}{$(6, \mathbb{1}, \mathbb{1})$} & $(\mathbf{2}, \mathbb{1}, \mathbb{1}, \mathbb{1})$ & & 1 & $\frac{1}{3}$ &,,+-+ &,,-+- & 0 & $-\frac{1}{3}$ & -1 & $-\frac{1}{3}$ & -1 & -10 & 0 & 0 \\
\hline & $(\mathbb{1}, \mathbf{4}, \mathbb{1}, \mathbb{1})$ & & 1 & $\frac{1}{3}$ &,,-+- &,,+-+ & 0 & $-\frac{1}{3}$ & -1 & $-\frac{1}{3}$ & -1 & 5 & 0 & 0 \\
\hline \multirow[t]{2}{*}{$(\overline{\mathbf{6}}, \mathbb{1}, \mathbb{1})$} & $(2, \mathbb{1}, \mathbb{1}, \mathbb{1})$ & & 1 & 1 &,,+-+ &,,-+- & $\frac{1}{2}$ & $\frac{1}{6}$ & 0 & $-\frac{1}{3}$ & -1 & 10 & 0 & 0 \\
\hline & $(\mathbb{1}, \overline{4}, \mathbb{1}, \mathbb{1})$ & & 1 & 1 &,,-+- &,,+-+ & $\frac{1}{2}$ & $\frac{1}{6}$ & 0 & $-\frac{1}{3}$ & -1 & -5 & 0 & 0 \\
\hline$(\mathbb{1}, \mathbb{1}, \mathbb{1})$ & $(\mathbb{1}, \mathbb{1}, \mathbb{1}, \mathbb{1})$ & $Y_{1}$ & 1 & $\frac{2}{3}$ &,,+-+ &,,-+- & 0 & $\frac{2}{3}$ & -2 & $-\frac{1}{3}$ & -1 & 0 & 0 & 0 \\
\hline$(\mathbb{1}, \mathbb{1}, \mathbb{1})$ & $(\mathbb{1}, \mathbb{1}, \mathbb{1}, \mathbb{1})$ & $\bar{Y}_{1}$ & 1 & $\frac{2}{3}$ &,,+-+ &,,-+- & $\frac{1}{2}$ & $-\frac{5}{6}$ & 1 & $-\frac{1}{3}$ & -1 & 0 & 0 & 0 \\
\hline \multirow[t]{2}{*}{$(\mathbb{1}, \mathbf{3}, \mathbb{1})$} & $(\mathbb{1}, \mathbb{1}, \mathbf{2}, \mathbb{1})$ & & 1 & $\frac{1}{3}$ &,,-+- &,,+-+ & $-\frac{1}{2}$ & $\frac{1}{6}$ & 1 & $\frac{2}{3}$ & 0 & 0 & 1 & 0 \\
\hline & $(\mathbb{1}, \mathbb{1}, \mathbb{1}, \mathbb{1})$ & & 1 & $\frac{1}{3}$ &,,+-+ &,,-+- & $-\frac{1}{2}$ & $\frac{1}{6}$ & 1 & $\frac{2}{3}$ & 0 & 0 & -2 & 0 \\
\hline \multirow[t]{2}{*}{$(\mathbb{1}, \overline{\mathbf{3}}, \mathbb{1})$} & $(\mathbb{1}, \mathbb{1}, \mathbf{2}, \mathbb{1})$ & & 1 & 1 &,,+-+ &,,-+- & $-\frac{1}{2}$ & $\frac{1}{6}$ & 1 & $-\frac{1}{3}$ & 1 & 0 & -1 & 0 \\
\hline & $(\mathbb{1}, \mathbb{1}, \mathbb{1}, \mathbb{1})$ & & 1 & 1 &,,-+- &,,+-+ & $-\frac{1}{2}$ & $\frac{1}{6}$ & 1 & $-\frac{1}{3}$ & 1 & 0 & 2 & 0 \\
\hline \multirow[t]{2}{*}{$\left(\mathbb{1}, \mathbb{1}, \mathbf{8}_{s}\right)$} & $(\mathbb{1}, \mathbb{1}, \mathbb{1}, \mathbf{4})$ & & 1 & $\frac{2}{3}$ &,,+-+ &,,-+- & $-\frac{1}{2}$ & $\frac{1}{6}$ & 1 & $\frac{1}{6}$ & $\frac{1}{2}$ & 0 & 0 & 1 \\
\hline & $(\mathbb{1}, \mathbb{1}, \mathbb{1}, \overline{\mathbf{4}})$ & & 1 & $\frac{2}{3}$ &,,-+- &,,+-+ & $-\frac{1}{2}$ & $\frac{1}{6}$ & 1 & $\frac{1}{6}$ & $\frac{1}{2}$ & 0 & 0 & -1 \\
\hline \multirow[t]{2}{*}{$(6, \mathbb{1}, \mathbb{1})$} & $(2, \mathbb{1}, \mathbb{1}, \mathbb{1})$ & & 2 & $\frac{1}{3}$ &,,-+- &,,+-+ & $\frac{1}{2}$ & $\frac{1}{6}$ & 0 & $-\frac{1}{3}$ & 1 & -10 & 0 & 0 \\
\hline & $(\mathbb{1}, \mathbf{4}, \mathbb{1}, \mathbb{1})$ & & 2 & $\frac{1}{3}$ &,,+-+ &,,-+- & $\frac{1}{2}$ & $\frac{1}{6}$ & 0 & $-\frac{1}{3}$ & 1 & 5 & 0 & 0 \\
\hline \multirow[t]{2}{*}{$(\overline{\mathbf{6}}, \mathbb{1}, \mathbb{1})$} & $(2, \mathbb{1}, \mathbb{1}, \mathbb{1})$ & & 2 & 1 &,,-+- &,,+-+ & 0 & $-\frac{1}{3}$ & 1 & $-\frac{1}{3}$ & 1 & 10 & 0 & 0 \\
\hline & $(\mathbb{1}, \overline{4}, \mathbb{1}, \mathbb{1})$ & & 2 & 1 &,,+-+ &,,-+- & 0 & $-\frac{1}{3}$ & 1 & $-\frac{1}{3}$ & 1 & -5 & 0 & 0 \\
\hline$(\mathbb{1}, \mathbb{1}, \mathbb{1})$ & $(\mathbb{1}, \mathbb{1}, \mathbb{1}, \mathbb{1})$ & $Y_{2}$ & 2 & $\frac{2}{3}$ &,,-+- &,,+-+ & 0 & $\frac{2}{3}$ & 2 & $-\frac{1}{3}$ & 1 & 0 & 0 & 0 \\
\hline$(\mathbb{1}, \mathbb{1}, \mathbb{1})$ & $(\mathbb{1}, \mathbb{1}, \mathbb{1}, \mathbb{1})$ & $\bar{Y}_{2}$ & 2 & $\frac{2}{3}$ &,,-+- &,,+-+ & $\frac{1}{2}$ & $-\frac{5}{6}$ & -1 & $-\frac{1}{3}$ & 1 & 0 & 0 & 0 \\
\hline \multirow[t]{2}{*}{$(\mathbb{1}, \mathbf{3}, \mathbb{1})$} & $(\mathbb{1}, \mathbb{1}, \mathbf{2}, \mathbb{1})$ & & 2 & $\frac{1}{3}$ &,,-+- &,,+-+ & $-\frac{1}{2}$ & $\frac{1}{6}$ & -1 & $-\frac{1}{3}$ & -1 & 0 & 1 & 0 \\
\hline & $(\mathbb{1}, \mathbb{1}, \mathbb{1}, \mathbb{1})$ & & 2 & $\frac{1}{3}$ &,,+-+ &,,-+- & $-\frac{1}{2}$ & $\frac{1}{6}$ & -1 & $-\frac{1}{3}$ & -1 & 0 & -2 & 0 \\
\hline \multirow[t]{2}{*}{$(\mathbb{1}, \overline{\mathbf{3}}, \mathbb{1})$} & $(\mathbb{1}, \mathbb{1}, \mathbf{2}, \mathbb{1})$ & & 2 & 1 &,,-+- &,,+-+ & $-\frac{1}{2}$ & $\frac{1}{6}$ & -1 & $\frac{2}{3}$ & 0 & 0 & -1 & 0 \\
\hline & $(\mathbb{1}, \mathbb{1}, \mathbb{1}, \mathbb{1})$ & & 2 & 1 &,,+-+ &,,-+- & $-\frac{1}{2}$ & $\frac{1}{6}$ & -1 & $\frac{2}{3}$ & 0 & 0 & 2 & 0 \\
\hline \multirow[t]{3}{*}{$\left(\mathbb{1}, \mathbb{1}, \boldsymbol{8}_{c}\right)$} & $(\mathbb{1}, \mathbb{1}, \mathbb{1}, \mathbf{6})$ & & 2 & $\frac{2}{3}$ &,,-+- &,,+-+ & $-\frac{1}{2}$ & $\frac{1}{6}$ & -1 & $\frac{1}{6}$ & $-\frac{1}{2}$ & 0 & 0 & 0 \\
\hline & $(\mathbb{1}, \mathbb{1}, \mathbb{1}, \mathbb{1})$ & & 2 & $\frac{2}{3}$ &,,+-+ &,,-+- & $-\frac{1}{2}$ & $\frac{1}{6}$ & -1 & $\frac{1}{6}$ & $-\frac{1}{2}$ & 0 & 0 & 2 \\
\hline & $(\mathbb{1}, \mathbb{1}, \mathbb{1}, \mathbb{1})$ & & 2 & $\frac{2}{3}$ &,,+-+ &,,-+- & $-\frac{1}{2}$ & $\frac{1}{6}$ & -1 & $\frac{1}{6}$ & $-\frac{1}{2}$ & 0 & 0 & -2 \\
\hline
\end{tabular}


Table 29 - continued from previous page

\begin{tabular}{c||c||c|c|c|c|c||c|c|c|c|c|c|c|c}
\hline Bulk & $n_{2}=1$ & $n_{3}$ & $\gamma_{3}^{\prime}$ & $H_{L}$ & $H_{R}$ & $t_{1}$ & $t_{2}$ & $t_{3}$ & $t_{4}$ & $t_{5}$ & $t_{6}^{1}$ & $t_{7}$ & $t_{8}$ \\
\hline
\end{tabular}
oscillator numbers to the fixed points $n_{2}=1$. For excited states see Table 28. The representations are given with respect to $\mathrm{SU}(6) \times[\mathrm{SU}(3) \times \mathrm{SO}(8)]$ and $\mathrm{SU}(2) \times \mathrm{SU}(4) \times\left[\mathrm{SU}(2)^{\prime} \times \mathrm{SU}(4)^{\prime}\right]$ in the bulk and at $n_{2}=1$, respectively. The three parities for the chiral multiplet components $H_{L}, H_{R}$ correspond to $\gamma=0, \frac{1}{2}, 1$. The localization in the $\mathrm{G}_{2}$-plane is given by $n_{6}=0$ for $\gamma=0$, and $n_{6}=2$ otherwise.

\section{E Zero modes in four dimensions}

The spectrum of the low-energy effective field theory in four dimensions follows from solutions of the mass equations (79), (80) and the projection conditions (144), (147). Up to the geometrical $\gamma^{\prime}$-phases, the result was already published in [13]. Here we present the list of chiral gauge multiplets of $\mathrm{SU}(3) \times \mathrm{SU}(2)$ with charges under nine linearly independent $\mathrm{U}(1)$ factors, with generators

$$
t_{1} \ldots, t_{5}, t_{6}^{0}, t_{7}, t_{8}, t_{Y}
$$

as defined in Table 4 . We additionally list the $B-L$ charges and the multiplet of the six-dimensional model which contains the zero mode. In the case of bulk multiplets, we chose to represent the latter by multiplets with respect to the local gauge group at $n_{2}=0$, rather than the bulk gauge group, since we are mainly interested in the effective $\mathrm{SU}(5)$ GUT at this fixed point in the main text. All left-handed chiral multiplets which arise from sectors $k=0,2,4$ are listed in Table 30, together with all left-handed chiral multiplets from $k=1,3,5$ at $\left(n_{2}, n_{2}^{\prime}\right)=(0,0),(1,0)$. Note that the spectrum of the sectors $k=1,3,5$ at $\left(n_{2}, n_{2}^{\prime}\right)=(0,1),(1,1)$ is an exact copy of the latter, and not shown explicitly. The $\mathrm{U}(1)$ charges with respect to the generators $t_{1}, \ldots, t_{5}$ can be inferred from the Tables $8,10,12$ and 26 .

\begin{tabular}{c||c|c|c|c|c|c||c|c|c|c|c||c|c} 
Multiplet & $k$ & $n_{2}$ & $n_{3}$ & $\gamma$ & $\gamma_{3}^{\prime}$ & $\gamma_{5}^{\prime}$ & $t_{6}^{0}$ & $t_{7}$ & $t_{8}$ & $t_{Y}$ & $t_{B-L}$ & \multicolumn{2}{|c}{ Origin } \\
\hline \hline$(\mathbf{3}, \mathbf{2}, \mathbb{1}, \mathbb{1})$ & 0 & 0 & 0 & 0 & 0 & 0 & 3 & 0 & 0 & $\frac{1}{6}$ & $\frac{1}{3}$ & $(\mathbf{1 0}, \mathbb{1}, \mathbb{1})$ & $\mathbf{1 0}_{(4)}$ \\
$(\overline{\mathbf{3}}, \mathbb{1}, \mathbb{1}, \mathbb{1})$ & 0 & 0 & 0 & 0 & 0 & 0 & 3 & 0 & 0 & $-\frac{2}{3}$ & $-\frac{1}{3}$ & $(\mathbf{1 0}, \mathbb{1}, \mathbb{1})$ & $\mathbf{1 0}_{(3)}$ \\
$(\mathbb{1}, \mathbb{1}, \mathbb{1}, \mathbb{1})$ & 0 & 0 & 0 & 0 & 0 & 0 & 3 & 0 & 0 & 1 & 1 & $(\mathbf{1 0}, \mathbb{1}, \mathbb{1})$ & $\mathbf{1 0}_{(3)}$ \\
\hline$(\mathbb{1}, \mathbf{2}, \mathbb{1}, \mathbb{1})$ & 0 & 0 & 0 & 0 & 0 & 0 & 6 & 0 & 0 & $-\frac{1}{2}$ & 0 & $(\overline{\mathbf{5}}, \mathbb{1}, \mathbb{1})$ & $\overline{\mathbf{5}}$ \\
$(\mathbb{1}, \mathbf{2}, \mathbb{1}, \mathbb{1})$ & 0 & 0 & 0 & 0 & 0 & 0 & -6 & 0 & 0 & $\frac{1}{2}$ & 0 & $(\mathbf{5}, \mathbb{1}, \mathbb{1})$ & $\mathbf{5}$ \\
$(\mathbb{1}, \mathbb{1}, \mathbb{1}, \overline{\mathbf{4}})$ & 0 & 0 & 0 & 0 & 0 & 0 & 0 & 0 & 1 & 0 & $\frac{1}{2}$ & $(\mathbb{1}, \mathbb{1}, \boldsymbol{8})$ & \\
$(\mathbb{1}, \mathbb{1}, \mathbb{1}, \overline{\mathbf{4}})$ & 0 & 0 & 0 & 0 & 0 & 0 & 0 & 0 & -1 & 0 & $\frac{1}{2}$ & $\left(\mathbb{1}, \mathbb{1}, \boldsymbol{8}_{s}\right)$ & \\
$(\mathbb{1}, \mathbb{1}, \mathbb{1}, \mathbb{1})$ & 0 & 0 & 0 & 0 & 0 & 0 & 0 & 0 & -2 & 0 & 0 & $\left(\mathbb{1}, \mathbb{1}, \boldsymbol{8}_{c}\right)$ &
\end{tabular}


Table 30 - continued from previous page

\begin{tabular}{|c|c|c|c|c|c|c|c|c|c|c|c|c|c|}
\hline Multiplet & $k$ & $n_{2}$ & $n_{3}$ & $\gamma$ & $\gamma_{3}^{\prime}$ & $\gamma_{5}^{\prime}$ & $t_{6}^{0}$ & $t_{7}$ & $t_{8}$ & $t_{Y}$ & $t_{B-L}$ & \multicolumn{2}{|c|}{ Origin } \\
\hline$(\mathbb{1}, \mathbb{1}, \mathbb{1}, \mathbb{1})$ & 0 & 0 & 0 & 0 & 0 & 0 & 0 & 0 & 2 & 0 & 0 & $\left(\mathbb{1}, \mathbb{1}, \boldsymbol{8}_{c}\right)$ & \\
\hline$(\mathbb{1}, \mathbb{1}, \mathbb{1}, \mathbb{1})$ & 0 & 0 & 0 & 0 & 0 & 0 & 0 & 0 & 0 & 0 & 0 & $(\mathbb{1}, \mathbb{1}, \mathbb{1})$ & $U_{1}^{c}$ \\
\hline$(3,2, \mathbb{1}, \mathbb{1})$ & 1 & 0 & 0 & 0 & 1 & 1 & $\frac{1}{2}$ & 0 & 0 & $\frac{1}{6}$ & $\frac{1}{3}$ & $(\mathbf{1 0}, \mathbb{1}, \mathbb{1})$ & $10_{(1)}$ \\
\hline$(\overline{\mathbf{3}}, \mathbb{1}, \mathbb{1}, \mathbb{1})$ & 1 & 0 & 0 & 0 & 1 & $\frac{1}{2}$ & $\frac{1}{2}$ & 0 & 0 & $-\frac{2}{3}$ & $-\frac{1}{3}$ & $(\mathbf{1 0}, \mathbb{1}, \mathbb{1})$ & $10_{(1)}$ \\
\hline$(\mathbb{1}, \mathbb{1}, \mathbb{1}, \mathbb{1})$ & 1 & 0 & 0 & 0 & 1 & $\frac{1}{2}$ & $\frac{1}{2}$ & 0 & 0 & 1 & 1 & $(\mathbf{1 0}, \mathbb{1}, \mathbb{1})$ & $10_{(1)}$ \\
\hline$(\overline{\mathbf{3}}, \mathbb{1}, \mathbb{1}, \mathbb{1})$ & 1 & 0 & 0 & 0 & $\frac{2}{3}$ & $\frac{1}{2}$ & $-\frac{3}{2}$ & 0 & 0 & $\frac{1}{3}$ & $-\frac{1}{3}$ & $(\overline{\mathbf{5}}, \mathbb{1}, \mathbb{1})$ & $\overline{\mathbf{5}}_{(1)}$ \\
\hline$(\mathbb{1}, 2, \mathbb{1}, \mathbb{1})$ & 1 & 0 & 0 & 0 & $\frac{2}{3}$ & 1 & $-\frac{3}{2}$ & 0 & 0 & $-\frac{1}{2}$ & -1 & $(\overline{\mathbf{5}}, \mathbb{1}, \mathbb{1})$ & $\overline{\mathbf{5}}_{(1)}$ \\
\hline$(\mathbb{1}, \mathbb{1}, \mathbb{1}, \mathbb{1})$ & 1 & 0 & 0 & 0 & $\frac{1}{3}$ & 1 & $\frac{5}{2}$ & 0 & 0 & 0 & -1 & $(\mathbb{1}, \mathbb{1}, \mathbb{1})$ & $N_{(1)}^{c}$ \\
\hline$(\mathbb{1}, \mathbb{1}, \mathbb{1}, \mathbb{1})$ & 1 & 0 & 0 & 0 & $\frac{1}{3}$ & $\frac{1}{2}$ & $\frac{5}{2}$ & 0 & 0 & 0 & 1 & $(\mathbb{1}, \mathbb{1}, \mathbb{1})$ & $S_{1}$ \\
\hline$(\mathbb{1}, \mathbb{1}, \mathbb{1}, \mathbb{1})$ & 1 & 0 & 0 & 0 & 1 & $\frac{1}{2}$ & $-\frac{5}{2}$ & 0 & 0 & 0 & 0 & $(\mathbb{1}, \mathbb{1}, \mathbb{1})$ & $S_{2}$ \\
\hline$(\mathbb{1}, \mathbb{1}, \mathbb{1}, \mathbb{1})$ & 1 & 0 & 0 & 0 & $\frac{1}{3}$ & $\frac{1}{2}$ & $\frac{5}{2}$ & 0 & 0 & 0 & 1 & $(\mathbb{1}, \mathbb{1}, \mathbb{1})$ & $S_{3}$ \\
\hline$(\mathbb{1}, \mathbb{1}, \mathbb{1}, \mathbb{1})$ & 1 & 0 & 0 & 0 & $\frac{1}{3}$ & $\frac{1}{2}$ & $\frac{5}{2}$ & 0 & 0 & 0 & 1 & $(\mathbb{1}, \mathbb{1}, \mathbb{1})$ & $S_{4}$ \\
\hline$(\mathbb{1}, \mathbb{1}, \mathbb{1}, \mathbb{1})$ & 1 & 0 & 0 & 0 & 1 & 1 & $-\frac{5}{2}$ & 0 & 0 & 0 & 0 & $(\mathbb{1}, \mathbb{1}, \mathbb{1})$ & $S_{5}$ \\
\hline$(\mathbb{1}, \mathbb{1}, \mathbb{1}, \mathbb{1})$ & 1 & 0 & 0 & 0 & 1 & 1 & $-\frac{5}{2}$ & 0 & 0 & 0 & 0 & $(\mathbb{1}, \mathbb{1}, \mathbb{1})$ & $S_{6}$ \\
\hline$(\mathbb{1}, \mathbb{1}, \mathbf{2}, \mathbb{1})$ & 1 & 0 & 1 & 0 & 1 & $\frac{1}{2}$ & $\frac{5}{2}$ & -1 & 0 & 0 & $\frac{1}{3}$ & $(\mathbb{1}, \overline{\mathbf{3}}, \mathbb{1})$ & \\
\hline$(\mathbb{1}, \mathbb{1}, \mathbb{1}, \mathbf{6})$ & 1 & 0 & 1 & 0 & $\frac{1}{3}$ & 1 & $\frac{5}{2}$ & 0 & 0 & 0 & 0 & $\left(\mathbb{1}, \mathbb{1}, \mathbf{8}_{c}\right)$ & \\
\hline$(\mathbb{1}, \mathbb{1}, \mathbb{1}, \mathbb{1})$ & 1 & 0 & 1 & 0 & 1 & 1 & $\frac{5}{2}$ & 2 & 0 & 0 & $\frac{1}{3}$ & $(\mathbb{1}, \overline{\mathbf{3}}, \mathbb{1})$ & \\
\hline$(\mathbb{1}, \mathbb{1}, \mathbb{1}, \mathbb{1})$ & 1 & 0 & 1 & 0 & $\frac{1}{3}$ & $\frac{1}{2}$ & $\frac{5}{2}$ & 0 & 0 & 0 & 0 & $(\mathbb{1}, \mathbb{1}, \mathbb{1})$ & $S_{7}$ \\
\hline$(\mathbb{1}, \mathbb{1}, \mathbb{1}, \mathbb{1})$ & 1 & 0 & 1 & 0 & $\frac{1}{3}$ & $\frac{1}{2}$ & $\frac{5}{2}$ & 0 & -2 & 0 & 0 & $\left(\mathbb{1}, \mathbb{1}, \boldsymbol{8}_{c}\right)$ & \\
\hline$(\mathbb{1}, \mathbb{1}, \mathbb{1}, \mathbb{1})$ & 1 & 0 & 1 & 0 & $\frac{1}{3}$ & $\frac{1}{2}$ & $\frac{5}{2}$ & 0 & 2 & 0 & 0 & $\left(\mathbb{1}, \mathbb{1}, \boldsymbol{8}_{c}\right)$ & \\
\hline$(\mathbb{1}, \mathbb{1}, \mathbf{2}, \mathbb{1})$ & 1 & 0 & 2 & 0 & $\frac{2}{3}$ & 1 & $\frac{5}{2}$ & 1 & 0 & 0 & $-\frac{1}{3}$ & $(\mathbb{1}, \mathbf{3}, \mathbb{1})$ & \\
\hline$(\mathbb{1}, \mathbb{1}, \mathbb{1}, \mathbb{1})$ & 1 & 0 & 2 & 0 & $\frac{2}{3}$ & $\frac{1}{2}$ & $\frac{5}{2}$ & -2 & 0 & 0 & $-\frac{1}{3}$ & $(\mathbb{1}, \mathbf{3}, \mathbb{1})$ & \\
\hline$(\mathbb{1}, \mathbb{1}, \mathbb{1}, \mathbb{1})$ & 1 & 0 & 2 & 0 & $\frac{1}{3}$ & $\frac{1}{2}$ & $\frac{5}{2}$ & 0 & 0 & 0 & -1 & $(\mathbb{1}, \mathbb{1}, \mathbb{1})$ & $S_{8}$ \\
\hline$(\mathbb{1}, 2, \mathbb{1}, \mathbb{1})$ & 1 & 1 & 0 & 0 & 1 & 1 & 0 & 1 & -1 & 0 & $-\frac{5}{6}$ & $(2, \mathbb{1}, \mathbb{1}, \mathbb{1})$ & \\
\hline$(\mathbb{1}, \mathbb{1}, \mathbb{1}, \mathbb{1})$ & 1 & 1 & 0 & 0 & $\frac{2}{3}$ & $\frac{1}{2}$ & 1 & 1 & -1 & $-\frac{1}{2}$ & $\frac{1}{6}$ & $(\mathbb{1}, \mathbb{1}, \mathbb{1}, \mathbb{1})$ & \\
\hline$(\mathbb{1}, \mathbb{1}, \mathbb{1}, \mathbb{1})$ & 1 & 1 & 0 & 0 & $\frac{1}{3}$ & $\frac{1}{2}$ & -1 & 1 & -1 & $\frac{1}{2}$ & $\frac{1}{6}$ & $(\mathbb{1}, \mathbb{1}, \mathbb{1}, \mathbb{1})$ & \\
\hline$(\mathbb{1}, 2, \mathbb{1}, \mathbb{1})$ & 1 & 1 & 1 & 0 & $\frac{1}{3}$ & $\frac{1}{2}$ & 0 & -1 & 1 & 0 & $\frac{5}{6}$ & $(2, \mathbb{1}, \mathbb{1}, \mathbb{1})$ & \\
\hline$(\mathbb{1}, \mathbb{1}, \mathbb{1}, \mathbb{1})$ & 1 & 1 & 1 & 0 & 1 & $\frac{1}{2}$ & 1 & -1 & 1 & $-\frac{1}{2}$ & $-\frac{1}{6}$ & $(\mathbb{1}, \mathbb{1}, \mathbb{1}, \mathbb{1})$ & \\
\hline$(\mathbb{1}, \mathbb{1}, \mathbb{1}, \mathbb{1})$ & 1 & 1 & 1 & 0 & $\frac{2}{3}$ & $\frac{1}{2}$ & -1 & -1 & 1 & $\frac{1}{2}$ & $-\frac{1}{6}$ & $(\mathbb{1}, \mathbb{1}, \mathbb{1}, \mathbb{1})$ & \\
\hline$(\mathbb{1}, 2, \mathbb{1}, \mathbb{1})$ & 1 & 1 & 2 & 0 & 1 & 1 & 0 & 1 & 1 & 0 & $\frac{1}{6}$ & $(2, \mathbb{1}, \mathbb{1}, \mathbb{1})$ & \\
\hline$(\mathbb{1}, 2, \mathbb{1}, \mathbb{1})$ & 1 & 1 & 2 & 0 & $\frac{1}{3}$ & $\frac{1}{2}$ & 0 & -1 & -1 & 0 & $-\frac{1}{6}$ & $(2, \mathbb{1}, \mathbb{1}, \mathbb{1})$ & \\
\hline$(\mathbb{1}, \mathbb{1}, \mathbb{1}, \mathbb{1})$ & 1 & 1 & 2 & 0 & 1 & $\frac{1}{2}$ & 1 & -1 & -1 & $-\frac{1}{2}$ & $-\frac{7}{6}$ & $(\mathbb{1}, \mathbb{1}, \mathbb{1}, \mathbb{1})$ & \\
\hline$(\mathbb{1}, \mathbb{1}, \mathbb{1}, \mathbb{1})$ & 1 & 1 & 2 & 0 & $\frac{2}{3}$ & 1 & 1 & 1 & 1 & $-\frac{1}{2}$ & $-\frac{5}{6}$ & $(\mathbb{1}, \mathbb{1}, \mathbb{1}, \mathbb{1})$ & \\
\hline$(\mathbb{1}, \mathbb{1}, \mathbb{1}, \mathbb{1})$ & 1 & 1 & 2 & 0 & $\frac{2}{3}$ & 1 & -1 & -1 & -1 & $\frac{1}{2}$ & $\frac{5}{6}$ & $(\mathbb{1}, \mathbb{1}, \mathbb{1}, \mathbb{1})$ & \\
\hline
\end{tabular}

Table 30 - continued on next page 
Table 30 - continued from previous page

\begin{tabular}{|c|c|c|c|c|c|c|c|c|c|c|c|c|c|}
\hline Multiplet & $k$ & $n_{2}$ & $n_{3}$ & $\gamma$ & $\gamma_{3}^{\prime}$ & $\gamma_{5}^{\prime}$ & $t_{6}^{0}$ & $t_{7}$ & $t_{8}$ & $t_{Y}$ & $t_{B-L}$ & \multicolumn{2}{|c|}{ Origin } \\
\hline$(\mathbb{1}, \mathbb{1}, \mathbb{1}, \mathbb{1})$ & 1 & 1 & 2 & 0 & $\frac{1}{3}$ & $\frac{1}{2}$ & -1 & 1 & 1 & $\frac{1}{2}$ & $\frac{7}{6}$ & $(\mathbb{1}, \mathbb{1}, \mathbb{1}, \mathbb{1})$ & \\
\hline$(3, \mathbb{1}, \mathbb{1}, \mathbb{1})$ & 2 & 0 & 0 & $\frac{1}{2}$ & $\frac{1}{3}$ & 0 & -1 & 0 & 0 & $-\frac{1}{3}$ & $-\frac{2}{3}$ & $(5, \mathbb{1}, \mathbb{1})$ & $\boldsymbol{5}_{0, \frac{1}{2}}$ \\
\hline$(\overline{\mathbf{3}}, \mathbb{1}, \mathbb{1}, \mathbb{1})$ & 2 & 0 & 0 & $\frac{1}{2}$ & 1 & 0 & 1 & 0 & 0 & $\frac{1}{3}$ & $\frac{2}{3}$ & $(\overline{\mathbf{5}}, \mathbb{1}, \mathbb{1})$ & $\overline{\mathbf{5}}_{0, \frac{1}{2}}$ \\
\hline$(\mathbb{1}, \mathbb{1}, \mathbb{1}, \mathbb{1})$ & 2 & 0 & 0 & $\frac{1}{2}$ & $\frac{1}{3}$ & 0 & 0 & -2 & 0 & 0 & $\frac{2}{3}$ & $(\mathbb{1}, \mathbf{3}, \mathbb{1})$ & \\
\hline$(\mathbb{1}, \mathbb{1}, \mathbf{2}, \mathbb{1})$ & 2 & 0 & 0 & $\frac{1}{2}$ & 1 & 0 & 0 & -1 & 0 & 0 & $\frac{1}{3}$ & $(\mathbb{1}, \overline{\mathbf{3}}, \mathbb{1})$ & \\
\hline$(\mathbb{1}, \mathbb{1}, \mathbb{1}, \overline{4})$ & 2 & 0 & 0 & $\frac{1}{2}$ & $\frac{2}{3}$ & 0 & 0 & 0 & 1 & 0 & $\frac{1}{2}$ & $(\mathbb{1}, \mathbb{1}, \mathbf{8})$ & \\
\hline$(\mathbb{1}, \mathbb{1}, \mathbb{1}, \mathbb{1})$ & 2 & 0 & 0 & 0 & $\frac{2}{3}$ & 0 & 0 & 0 & 0 & 0 & 1 & $(\mathbb{1}, \mathbb{1}, \mathbb{1})$ & $Y_{0,0}$ \\
\hline$(\mathbb{1}, \mathbb{1}, \mathbb{1}, \mathbb{1})$ & 2 & 0 & 0 & 0 & $\frac{2}{3}$ & 0 & 0 & 0 & 0 & 0 & -1 & $(\mathbb{1}, \mathbb{1}, \mathbb{1})$ & $\bar{Y}_{0,0}$ \\
\hline$(\mathbb{1}, \mathbb{1}, \mathbb{1}, \mathbb{1})$ & 2 & 0 & 0 & 1 & $\frac{2}{3}$ & 0 & 0 & 0 & 0 & 0 & 1 & $(\mathbb{1}, \mathbb{1}, \mathbb{1})$ & $Y_{0,1}$ \\
\hline$(\mathbb{1}, \mathbb{1}, \mathbb{1}, \mathbb{1})$ & 2 & 0 & 0 & 1 & $\frac{2}{3}$ & 0 & 0 & 0 & 0 & 0 & -1 & $(\mathbb{1}, \mathbb{1}, \mathbb{1})$ & $\bar{Y}_{0,1}$ \\
\hline$(\mathbb{1}, \mathbf{2}, \mathbb{1}, \mathbb{1})$ & 2 & 0 & 1 & 0 & $\frac{1}{3}$ & 0 & -1 & 0 & 0 & $\frac{1}{2}$ & 0 & $(5, \mathbb{1}, \mathbb{1})$ & $\mathbf{5}_{1,0}$ \\
\hline$(\mathbb{1}, \mathbf{2}, \mathbb{1}, \mathbb{1})$ & 2 & 0 & 1 & 0 & 1 & 0 & 1 & 0 & 0 & $-\frac{1}{2}$ & 0 & $(\overline{\mathbf{5}}, \mathbb{1}, \mathbb{1})$ & $\overline{\mathbf{5}}_{1,0}$ \\
\hline$(\mathbb{1}, 2, \mathbb{1}, \mathbb{1})$ & 2 & 0 & 1 & 1 & $\frac{1}{3}$ & 0 & -1 & 0 & 0 & $\frac{1}{2}$ & 0 & $(5, \mathbb{1}, \mathbb{1})$ & $\mathbf{5}_{1,1}$ \\
\hline$(\mathbb{1}, 2, \mathbb{1}, \mathbb{1})$ & 2 & 0 & 1 & 1 & 1 & 0 & 1 & 0 & 0 & $-\frac{1}{2}$ & 0 & $(\overline{\mathbf{5}}, \mathbb{1}, \mathbb{1})$ & $\overline{\mathbf{5}}_{1,1}$ \\
\hline$(\mathbb{1}, \mathbb{1}, \mathbf{2}, \mathbb{1})$ & 2 & 0 & 1 & $\frac{1}{2}$ & $\frac{1}{3}$ & 0 & 0 & 1 & 0 & 0 & $-\frac{1}{3}$ & $(\mathbb{1}, \mathbf{3}, \mathbb{1})$ & \\
\hline$(\mathbb{1}, \mathbb{1}, \mathbb{1}, \mathbb{1})$ & 2 & 0 & 1 & $\frac{1}{2}$ & 1 & 0 & 0 & 2 & 0 & 0 & $-\frac{2}{3}$ & $(\mathbb{1}, \overline{\mathbf{3}}, \mathbb{1})$ & \\
\hline$(\mathbb{1}, \mathbb{1}, \mathbb{1}, \mathbf{4})$ & 2 & 0 & 1 & 0 & $\frac{2}{3}$ & 0 & 0 & 0 & 1 & 0 & $-\frac{1}{2}$ & $\left(\mathbb{1}, \mathbb{1}, \mathbf{8}_{s}\right)$ & \\
\hline$(\mathbb{1}, \mathbb{1}, \mathbb{1}, \mathbf{4})$ & 2 & 0 & 1 & 1 & $\frac{2}{3}$ & 0 & 0 & 0 & 1 & 0 & $-\frac{1}{2}$ & $\left(\mathbb{1}, \mathbb{1}, \mathbf{8}_{s}\right)$ & \\
\hline$(\mathbb{1}, \mathbb{1}, \mathbb{1}, \mathbb{1})$ & 2 & 0 & 1 & $\frac{1}{2}$ & $\frac{1}{3}$ & 0 & 5 & 0 & 0 & 0 & 0 & $(\mathbb{1}, \mathbb{1}, \mathbb{1})$ & $X_{1, \frac{1}{2}}$ \\
\hline$(\mathbb{1}, \mathbb{1}, \mathbb{1}, \mathbb{1})$ & 2 & 0 & 1 & $\frac{1}{2}$ & 1 & 0 & -5 & 0 & 0 & 0 & 0 & $(\mathbb{1}, \mathbb{1}, \mathbb{1})$ & $\bar{X}_{1, \frac{1}{2}}$ \\
\hline$(\mathbb{1}, \mathbb{1}, \mathbb{1}, \mathbb{1})$ & 2 & 0 & 1 & 0 & $\frac{2}{3}$ & 0 & 0 & 0 & 0 & 0 & 1 & $(\mathbb{1}, \mathbb{1}, \mathbb{1})$ & $Y_{1,0}$ \\
\hline$(\mathbb{1}, \mathbb{1}, \mathbb{1}, \mathbb{1})$ & 2 & 0 & 1 & 0 & $\frac{2}{3}$ & 0 & 0 & 0 & 0 & 0 & -1 & $(\mathbb{1}, \mathbb{1}, \mathbb{1})$ & $\bar{Y}_{1,0}$ \\
\hline$(\mathbb{1}, \mathbb{1}, \mathbb{1}, \mathbb{1})$ & 2 & 0 & 1 & 1 & $\frac{2}{3}$ & 0 & 0 & 0 & 0 & 0 & 1 & $(\mathbb{1}, \mathbb{1}, \mathbb{1})$ & $Y_{1,1}$ \\
\hline$(\mathbb{1}, \mathbb{1}, \mathbb{1}, \mathbb{1})$ & 2 & 0 & 1 & 1 & $\frac{2}{3}$ & 0 & 0 & 0 & 0 & 0 & -1 & $(\mathbb{1}, \mathbb{1}, \mathbb{1})$ & $\bar{Y}_{1,1}$ \\
\hline$(\overline{\mathbf{3}}, \mathbb{1}, \mathbb{1}, \mathbb{1})$ & 2 & 0 & 2 & 0 & 1 & 0 & 1 & 0 & 0 & $\frac{1}{3}$ & $-\frac{1}{3}$ & $(\overline{\mathbf{5}}, \mathbb{1}, \mathbb{1})$ & $\overline{\mathbf{5}}_{2,0}$ \\
\hline$(\overline{\mathbf{3}}, \mathbb{1}, \mathbb{1}, \mathbb{1})$ & 2 & 0 & 2 & 1 & 1 & 0 & 1 & 0 & 0 & $\frac{1}{3}$ & $-\frac{1}{3}$ & $(\overline{\mathbf{5}}, \mathbb{1}, \mathbb{1})$ & $\overline{\mathbf{5}}_{2,1}$ \\
\hline$(\mathbb{1}, \mathbf{2}, \mathbb{1}, \mathbb{1})$ & 2 & 0 & 2 & $\frac{1}{2}$ & $\frac{1}{3}$ & 0 & -1 & 0 & 0 & $\frac{1}{2}$ & 1 & $(5, \mathbb{1}, \mathbb{1})$ & $\mathbf{5}_{2, \frac{1}{2}}$ \\
\hline$(\mathbb{1}, \mathbb{1}, \mathbb{1}, 6)$ & 2 & 0 & 2 & $\frac{1}{2}$ & $\frac{2}{3}$ & 0 & 0 & 0 & 0 & 0 & 0 & $\left(\mathbb{1}, \mathbb{1}, \boldsymbol{8}_{c}\right)$ & \\
\hline$(\mathbb{1}, \mathbb{1}, \mathbb{1}, \mathbb{1})$ & 2 & 0 & 2 & 0 & 1 & 0 & 0 & 2 & 0 & 0 & $\frac{1}{3}$ & $(\mathbb{1}, \overline{\mathbf{3}}, \mathbb{1})$ & \\
\hline$(\mathbb{1}, \mathbb{1}, \mathbb{1}, \mathbb{1})$ & 2 & 0 & 2 & 0 & $\frac{1}{3}$ & 0 & 0 & -2 & 0 & 0 & $-\frac{1}{3}$ & $(\mathbb{1}, \mathbf{3}, \mathbb{1})$ & \\
\hline$(\mathbb{1}, \mathbb{1}, \mathbb{1}, \mathbb{1})$ & 2 & 0 & 2 & 1 & 1 & 0 & 0 & 2 & 0 & 0 & $\frac{1}{3}$ & $(\mathbb{1}, \overline{\mathbf{3}}, \mathbb{1})$ & \\
\hline$(\mathbb{1}, \mathbb{1}, \mathbb{1}, \mathbb{1})$ & 2 & 0 & 2 & 1 & $\frac{1}{3}$ & 0 & 0 & -2 & 0 & 0 & $-\frac{1}{3}$ & $(\mathbb{1}, \mathbf{3}, \mathbb{1})$ & \\
\hline$(\mathbb{1}, \mathbb{1}, \mathbb{1}, \mathbb{1})$ & 2 & 0 & 2 & 0 & $\frac{1}{3}$ & 0 & 5 & 0 & 0 & 0 & 1 & $(\mathbb{1}, \mathbb{1}, \mathbb{1})$ & $X_{2,0}$ \\
\hline$(\mathbb{1}, \mathbb{1}, \mathbb{1}, \mathbb{1})$ & 2 & 0 & 2 & 1 & $\frac{1}{3}$ & 0 & 5 & 0 & 0 & 0 & 1 & $(\mathbb{1}, \mathbb{1}, \mathbb{1})$ & $X_{2,1}$ \\
\hline
\end{tabular}


Table 30 - continued from previous page

\begin{tabular}{|c|c|c|c|c|c|c|c|c|c|c|c|c|c|}
\hline Multiplet & $k$ & $n_{2}$ & $n_{3}$ & $\gamma$ & $\gamma_{3}^{\prime}$ & $\gamma_{5}^{\prime}$ & $t_{6}^{0}$ & $t_{7}$ & $t_{8}$ & $t_{Y}$ & $t_{B-L}$ & \multicolumn{2}{|c|}{ Origin } \\
\hline$(\mathbb{1}, \mathbb{1}, \mathbb{1}, \mathbb{1})$ & 2 & 0 & 2 & $\frac{1}{2}$ & $\frac{2}{3}$ & 0 & 0 & 0 & 0 & 0 & 0 & $(\mathbb{1}, \mathbb{1}, \mathbb{1})$ & $\bar{Y}_{2, \frac{1}{2}}$ \\
\hline$(\mathbb{1}, \mathbb{1}, \mathbf{2}, \mathbb{1})$ & 3 & 0 & 0 & $\frac{1}{3}$ & 0 & $\frac{1}{2}$ & $\frac{5}{2}$ & 1 & 0 & 0 & $-\frac{1}{3}$ & $(\mathbb{1}, \mathbf{3}, \mathbb{1})$ & \\
\hline$(\mathbb{1}, \mathbb{1}, \mathbf{2}, \mathbb{1})$ & 3 & 0 & 0 & $\frac{1}{3}$ & 0 & $\frac{1}{2}$ & $-\frac{5}{2}$ & -1 & 0 & 0 & $\frac{1}{3}$ & $(\mathbb{1}, \overline{\mathbf{3}}, \mathbb{1})$ & \\
\hline$(\mathbb{1}, \mathbb{1}, \mathbb{1}, \mathbb{1})$ & 3 & 0 & 0 & $\frac{1}{3}$ & 0 & 1 & $\frac{5}{2}$ & -2 & 0 & 0 & $-\frac{1}{3}$ & $(\mathbb{1}, \mathbf{3}, \mathbb{1})$ & \\
\hline$(\mathbb{1}, \mathbb{1}, \mathbb{1}, \mathbb{1})$ & 3 & 0 & 0 & $\frac{1}{3}$ & 0 & 1 & $-\frac{5}{2}$ & 2 & 0 & 0 & $\frac{1}{3}$ & $(\mathbb{1}, \overline{\mathbf{3}}, \mathbb{1})$ & \\
\hline$(\mathbb{1}, \mathbb{1}, \mathbb{1}, \mathbb{1})$ & 3 & 1 & 0 & 0 & 0 & $\frac{1}{2}$ & 1 & 1 & -1 & $-\frac{1}{2}$ & $-\frac{5}{6}$ & $(\mathbb{1}, \mathbb{1}, \mathbb{1}, \mathbb{1})$ & \\
\hline$(\mathbb{1}, \mathbb{1}, \mathbb{1}, \mathbb{1})$ & 3 & 1 & 0 & 0 & 0 & $\frac{1}{2}$ & -1 & -1 & 1 & $\frac{1}{2}$ & $\frac{5}{6}$ & $(\mathbb{1}, \mathbb{1}, \mathbb{1}, \mathbb{1})$ & \\
\hline$(\mathbb{1}, \mathbb{1}, \mathbb{1}, \mathbb{1})$ & 3 & 1 & 0 & $\frac{1}{3}$ & 0 & 1 & 1 & 1 & -1 & $-\frac{1}{2}$ & $\frac{7}{6}$ & $(\mathbb{1}, \mathbb{1}, \mathbb{1}, \mathbb{1})$ & \\
\hline$(\mathbb{1}, \mathbb{1}, \mathbb{1}, \mathbb{1})$ & 3 & 1 & 0 & $\frac{1}{3}$ & 0 & 1 & -1 & -1 & 1 & $\frac{1}{2}$ & $-\frac{7}{6}$ & $(\mathbb{1}, \mathbb{1}, \mathbb{1}, \mathbb{1})$ & \\
\hline$(\mathbb{1}, \mathbb{1}, \mathbb{1}, \mathbb{1})$ & 3 & 1 & 0 & $\frac{2}{3}$ & 0 & $\frac{1}{2}$ & 1 & 1 & -1 & $-\frac{1}{2}$ & $-\frac{5}{6}$ & $(\mathbb{1}, \mathbb{1}, \mathbb{1}, \mathbb{1})$ & \\
\hline$(\mathbb{1}, \mathbb{1}, \mathbb{1}, \mathbb{1})$ & 3 & 1 & 0 & $\frac{2}{3}$ & 0 & $\frac{1}{2}$ & -1 & -1 & 1 & $\frac{1}{2}$ & $\frac{5}{6}$ & $(\mathbb{1}, \mathbb{1}, \mathbb{1}, \mathbb{1})$ & \\
\hline$(\mathbb{1}, \mathbb{1}, \mathbb{1}, \mathbb{1})$ & 3 & 1 & 0 & 1 & 0 & $\frac{1}{2}$ & 1 & 1 & -1 & $-\frac{1}{2}$ & $-\frac{5}{6}$ & $(\mathbb{1}, \mathbb{1}, \mathbb{1}, \mathbb{1})$ & \\
\hline$(\mathbb{1}, \mathbb{1}, \mathbb{1}, \mathbb{1})$ & 3 & 1 & 0 & 1 & 0 & $\frac{1}{2}$ & -1 & -1 & 1 & $\frac{1}{2}$ & $\frac{5}{6}$ & $(\mathbb{1}, \mathbb{1}, \mathbb{1}, \mathbb{1})$ & \\
\hline$(3, \mathbb{1}, \mathbb{1}, \mathbb{1})$ & 4 & 0 & 0 & 0 & 1 & 0 & -1 & 0 & 0 & $-\frac{1}{3}$ & $-\frac{2}{3}$ & $(5, \mathbb{1}, \mathbb{1})$ & $\overline{\mathbf{5}}_{0,0}^{c}$ \\
\hline$(3, \mathbb{1}, \mathbb{1}, \mathbb{1})$ & 4 & 0 & 0 & 1 & 1 & 0 & -1 & 0 & 0 & $-\frac{1}{3}$ & $-\frac{2}{3}$ & $(5, \mathbb{1}, \mathbb{1})$ & $\overline{\mathbf{5}}_{0,1}^{c}$ \\
\hline$(\overline{\mathbf{3}}, \mathbb{1}, \mathbb{1}, \mathbb{1})$ & 4 & 0 & 0 & 0 & $\frac{1}{3}$ & 0 & 1 & 0 & 0 & $\frac{1}{3}$ & $\frac{2}{3}$ & $(\overline{\mathbf{5}}, \mathbb{1}, \mathbb{1})$ & $\mathbf{5}_{0,0}^{c}$ \\
\hline$(\overline{3}, \mathbb{1}, \mathbb{1}, \mathbb{1})$ & 4 & 0 & 0 & 1 & $\frac{1}{3}$ & 0 & 1 & 0 & 0 & $\frac{1}{3}$ & $\frac{2}{3}$ & $(\overline{\mathbf{5}}, \mathbb{1}, \mathbb{1})$ & $\mathbf{5}_{0,1}^{c}$ \\
\hline$(\mathbb{1}, \mathbb{1}, \mathbf{2}, \mathbb{1})$ & 4 & 0 & 0 & 0 & 1 & 0 & 0 & 1 & 0 & 0 & $-\frac{1}{3}$ & $(\mathbb{1}, \mathbf{3}, \mathbb{1})$ & \\
\hline$(\mathbb{1}, \mathbb{1}, \mathbf{2}, \mathbb{1})$ & 4 & 0 & 0 & 1 & 1 & 0 & 0 & 1 & 0 & 0 & $-\frac{1}{3}$ & $(\mathbb{1}, \mathbf{3}, \mathbb{1})$ & \\
\hline$(\mathbb{1}, \mathbb{1}, \mathbb{1}, 4)$ & 4 & 0 & 0 & 0 & $\frac{2}{3}$ & 0 & 0 & 0 & -1 & 0 & $-\frac{1}{2}$ & $(\mathbb{1}, \mathbb{1}, \mathbf{8})$ & \\
\hline$(\mathbb{1}, \mathbb{1}, \mathbb{1}, \mathbf{4})$ & 4 & 0 & 0 & 1 & $\frac{2}{3}$ & 0 & 0 & 0 & -1 & 0 & $-\frac{1}{2}$ & $(\mathbb{1}, \mathbb{1}, \mathbf{8})$ & \\
\hline$(\mathbb{1}, \mathbb{1}, \mathbb{1}, \mathbb{1})$ & 4 & 0 & 0 & 0 & $\frac{1}{3}$ & 0 & 0 & 2 & 0 & 0 & $-\frac{2}{3}$ & $(\mathbb{1}, \overline{\mathbf{3}}, \mathbb{1})$ & \\
\hline$(\mathbb{1}, \mathbb{1}, \mathbb{1}, \mathbb{1})$ & 4 & 0 & 0 & 1 & $\frac{1}{3}$ & 0 & 0 & 2 & 0 & 0 & $-\frac{2}{3}$ & $(\mathbb{1}, \overline{\mathbf{3}}, \mathbb{1})$ & \\
\hline$(\mathbb{1}, \mathbb{1}, \mathbb{1}, \mathbb{1})$ & 4 & 0 & 0 & $\frac{1}{2}$ & $\frac{2}{3}$ & 0 & 0 & 0 & 0 & 0 & -1 & $(\mathbb{1}, \mathbb{1}, \mathbb{1})$ & $Y_{0, \frac{1}{2}}^{c}$ \\
\hline$(\mathbb{1}, \mathbb{1}, \mathbb{1}, \mathbb{1})$ & 4 & 0 & 0 & $\frac{1}{2}$ & $\frac{2}{3}$ & 0 & 0 & 0 & 0 & 0 & 1 & $(\mathbb{1}, \mathbb{1}, \mathbb{1})$ & $\bar{Y}_{0, \frac{1}{2}}^{c}$ \\
\hline$(\mathbb{1}, 2, \mathbb{1}, \mathbb{1})$ & 4 & 0 & 1 & $\frac{1}{2}$ & 1 & 0 & -1 & 0 & 0 & $\frac{1}{2}$ & 0 & $(5, \mathbb{1}, \mathbb{1})$ & $\overline{\mathbf{5}}_{1, \frac{1}{2}}^{c}$ \\
\hline$(\mathbb{1}, 2, \mathbb{1}, \mathbb{1})$ & 4 & 0 & 1 & $\frac{1}{2}$ & $\frac{1}{3}$ & 0 & 1 & 0 & 0 & $-\frac{1}{2}$ & 0 & $(\overline{5}, \mathbb{1}, \mathbb{1})$ & $\mathbf{5}_{1, \frac{1}{2}}^{c}$ \\
\hline$(\mathbb{1}, \mathbb{1}, \mathbf{2}, \mathbb{1})$ & 4 & 0 & 1 & 0 & $\frac{1}{3}$ & 0 & 0 & -1 & 0 & 0 & $\frac{1}{3}$ & $(\mathbb{1}, \overline{\mathbf{3}}, \mathbb{1})$ & \\
\hline$(\mathbb{1}, \mathbb{1}, \mathbf{2}, \mathbb{1})$ & 4 & 0 & 1 & 1 & $\frac{1}{3}$ & 0 & 0 & -1 & 0 & 0 & $\frac{1}{3}$ & $(\mathbb{1}, \overline{\mathbf{3}}, \mathbb{1})$ & \\
\hline$(\mathbb{1}, \mathbb{1}, \mathbb{1}, \overline{\mathbf{4}})$ & 4 & 0 & 1 & $\frac{1}{2}$ & $\frac{2}{3}$ & 0 & 0 & 0 & -1 & 0 & $\frac{1}{2}$ & $\left(\mathbb{1}, \mathbb{1}, \boldsymbol{8}_{s}\right)$ & \\
\hline$(\mathbb{1}, \mathbb{1}, \mathbb{1}, \mathbb{1})$ & 4 & 0 & 1 & 0 & 1 & 0 & 0 & -2 & 0 & 0 & $\frac{2}{3}$ & $(\mathbb{1}, \mathbf{3}, \mathbb{1})$ & \\
\hline$(\mathbb{1}, \mathbb{1}, \mathbb{1}, \mathbb{1})$ & 4 & 0 & 1 & 1 & 1 & 0 & 0 & -2 & 0 & 0 & $\frac{2}{3}$ & $(\mathbb{1}, \mathbf{3}, \mathbb{1})$ & \\
\hline$(\mathbb{1}, \mathbb{1}, \mathbb{1}, \mathbb{1})$ & 4 & 0 & 1 & 0 & 1 & 0 & 5 & 0 & 0 & 0 & 0 & $(\mathbb{1}, \mathbb{1}, \mathbb{1})$ & $\bar{X}_{1,0}^{c}$ \\
\hline$(\mathbb{1}, \mathbb{1}, \mathbb{1}, \mathbb{1})$ & 4 & 0 & 1 & 0 & $\frac{1}{3}$ & 0 & -5 & 0 & 0 & 0 & 0 & $(\mathbb{1}, \mathbb{1}, \mathbb{1})$ & $X_{1,0}^{c}$ \\
\hline
\end{tabular}


Table 30 - continued from previous page

\begin{tabular}{c||c|c|c|c|c|c||c|c|c|c|c||c|c} 
Multiplet & $k$ & $n_{2}$ & $n_{3}$ & $\gamma$ & $\gamma_{3}^{\prime}$ & $\gamma_{5}^{\prime}$ & $t_{6}^{0}$ & $t_{7}$ & $t_{8}$ & $t_{Y}$ & $t_{B-L}$ & \multicolumn{2}{|c}{ Origin } \\
\hline \hline$(\mathbb{1}, \mathbb{1}, \mathbb{1}, \mathbb{1})$ & 4 & 0 & 1 & 1 & 1 & 0 & 5 & 0 & 0 & 0 & 0 & $(\mathbb{1}, \mathbb{1}, \mathbb{1})$ & $\bar{X}_{1,1}^{c}$ \\
$(\mathbb{1}, \mathbb{1}, \mathbb{1}, \mathbb{1})$ & 4 & 0 & 1 & 1 & $\frac{1}{3}$ & 0 & -5 & 0 & 0 & 0 & 0 & $(\mathbb{1}, \mathbb{1}, \mathbb{1})$ & $X_{1,1}^{c}$ \\
$(\mathbb{1}, \mathbb{1}, \mathbb{1}, \mathbb{1})$ & 4 & 0 & 1 & $\frac{1}{2}$ & $\frac{2}{3}$ & 0 & 0 & 0 & 0 & 0 & -1 & $(\mathbb{1}, \mathbb{1}, \mathbb{1})$ & $Y_{1, \frac{1}{2}}^{c}$ \\
$(\mathbb{1}, \mathbb{1}, \mathbb{1}, \mathbb{1})$ & 4 & 0 & 1 & $\frac{1}{2}$ & $\frac{2}{3}$ & 0 & 0 & 0 & 0 & 0 & 1 & $(\mathbb{1}, \mathbb{1}, \mathbb{1})$ & $\bar{Y}_{1, \frac{1}{2}}^{c}$ \\
\hline$(\mathbf{3}, \mathbb{1}, \mathbb{1}, \mathbb{1})$ & 4 & 0 & 2 & $\frac{1}{2}$ & 1 & 0 & -1 & 0 & 0 & $-\frac{1}{3}$ & $\frac{1}{3}$ & $(\mathbf{5}, \mathbb{1}, \mathbb{1})$ & $\overline{\mathbf{5}}_{2, \frac{1}{2}}^{c}$ \\
$(\mathbb{1}, \mathbf{2}, \mathbb{1}, \mathbb{1})$ & 4 & 0 & 2 & 0 & $\frac{1}{3}$ & 0 & 1 & 0 & 0 & $-\frac{1}{2}$ & -1 & $(\overline{\mathbf{5}}, \mathbb{1}, \mathbb{1})$ & $\mathbf{5}_{2,0}^{c}$ \\
$(\mathbb{1}, \mathbf{2}, \mathbb{1}, \mathbb{1})$ & 4 & 0 & 2 & 1 & $\frac{1}{3}$ & 0 & 1 & 0 & 0 & $-\frac{1}{2}$ & -1 & $(\overline{\mathbf{5}}, \mathbb{1}, \mathbb{1})$ & $\mathbf{5}_{2,1}^{c}$ \\
$(\mathbb{1}, \mathbb{1}, \mathbb{1}, \mathbf{6})$ & 4 & 0 & 2 & 0 & $\frac{2}{3}$ & 0 & 0 & 0 & 0 & 0 & 0 & $\left(\mathbb{1}, \mathbb{1}, \mathbf{8}_{c}\right)$ & \\
$(\mathbb{1}, \mathbb{1}, \mathbb{1}, \mathbf{6})$ & 4 & 0 & 2 & 1 & $\frac{2}{3}$ & 0 & 0 & 0 & 0 & 0 & 0 & $\left(\mathbb{1}, \mathbb{1}, \mathbf{8}_{c}\right)$ & \\
$(\mathbb{1}, \mathbb{1}, \mathbb{1}, \mathbb{1})$ & 4 & 0 & 2 & $\frac{1}{2}$ & 1 & 0 & 0 & -2 & 0 & 0 & $-\frac{1}{3}$ & $(\mathbb{1}, \mathbf{3}, \mathbb{1})$ & \\
$(\mathbb{1}, \mathbb{1}, \mathbb{1}, \mathbb{1})$ & 4 & 0 & 2 & $\frac{1}{2}$ & $\frac{1}{3}$ & 0 & 0 & 2 & 0 & 0 & $\frac{1}{3}$ & $(\mathbb{1}, \overline{\mathbf{3}}, \mathbb{1})$ & \\
$(\mathbb{1}, \mathbb{1}, \mathbb{1}, \mathbb{1})$ & 4 & 0 & 2 & $\frac{1}{2}$ & $\frac{1}{3}$ & 0 & -5 & 0 & 0 & 0 & -1 & $(\mathbb{1}, \mathbb{1}, \mathbb{1})$ & $X_{2, \frac{1}{2}}^{c}$ \\
$(\mathbb{1}, \mathbb{1}, \mathbb{1}, \mathbb{1})$ & 4 & 0 & 2 & 0 & $\frac{2}{3}$ & 0 & 0 & 0 & 0 & 0 & 0 & $(\mathbb{1}, \mathbb{1}, \mathbb{1})$ & $\bar{X}_{2,0}^{c}$ \\
$(\mathbb{1}, \mathbb{1}, \mathbb{1}, \mathbb{1})$ & 4 & 0 & 2 & 1 & $\frac{2}{3}$ & 0 & 0 & 0 & 0 & 0 & 0 & $(\mathbb{1}, \mathbb{1}, \mathbb{1})$ & $\bar{X}_{2,1}^{c}$ \\
& \multicolumn{10}{|c|}{ Table $30:$ Zero modes in four dimensions and their origin. }
\end{tabular}

\section{F Discrete symmetry transformations}

In Chapter 4 three vacua were studied in detail,

$$
\begin{aligned}
& \mathcal{S}_{0}=\left\{X_{0}, \bar{X}_{0}^{c}, U_{2}, U_{4}, S_{2}, S_{5}\right\}, \\
& \mathcal{S}_{1}=\mathcal{S}_{0} \cup\left\{X_{1}, \bar{X}_{1}, Y_{2}, S_{7}\right\}, \\
& \mathcal{S}_{2}=\mathcal{S}_{0} \cup\left\{X_{1}^{c}, \bar{X}_{1}, Y_{2}^{c}, \bar{Y}_{2}, U_{1}^{c}, U_{3}, S_{6}, S_{7}\right\},
\end{aligned}
$$

with unbroken symmetries

$$
\begin{aligned}
G_{\text {vac }}\left(\mathcal{S}_{0}\right) & =\left[\tilde{\mathbb{Z}}_{24} \times \mathbb{Z}_{6}\right]_{R} \times \mathrm{U}(1)_{5} \times \mathbb{Z}_{120}, \\
G_{\text {vac }}\left(\mathcal{S}_{1}\right) & =\left[\mathbb{Z}_{6} \times \tilde{\mathbb{Z}}_{2}\right]_{R} \times \mathbb{Z}_{60} \times \mathbb{Z}_{2}, \\
G_{\text {vac }}\left(\mathcal{S}_{2}\right) & =\left[\tilde{\mathbb{Z}}_{4} \times \mathbb{Z}_{2}\right]_{R} \times \mathbb{Z}_{60},
\end{aligned}
$$

under which a field $\phi$ transforms as

$$
\phi \mapsto e^{2 \pi i \eta_{\mathcal{S}}(\phi)} \phi, \quad \eta_{\mathcal{S}}(\phi)=\alpha_{\mathcal{S}}^{\prime} \cdot \mathbf{Q}(\phi)+\mathbf{r}_{\mathcal{S}}^{\prime} \cdot \mathcal{K}(\phi),
$$




\begin{tabular}{c||c|c|c|c||c|c|c|c|c|c|c|c||c} 
Multiplet & $k$ & $n_{3}$ & $\gamma$ & $\gamma_{3}^{\prime}$ & $t_{1}$ & $t_{2}$ & $t_{3}$ & $t_{4}$ & $t_{5}$ & $t_{6}$ & $t_{7}$ & $t_{8}$ & $t_{Y}$ \\
\hline \hline$(\mathbf{2}, \mathbb{1}, \mathbb{1}, \mathbb{1})$ & 1 & 0 & 0 & 1 & $-\frac{1}{2}$ & $-\frac{1}{6}$ & 0 & $-\frac{5}{12}$ & $\frac{1}{4}$ & 0 & 1 & -1 & 0 \\
$(\mathbb{1}, \mathbb{1}, \mathbb{1}, \mathbb{1})$ & 1 & 0 & 0 & $\frac{2}{3}$ & $\frac{1}{2}$ & $-\frac{1}{6}$ & -1 & $-\frac{5}{12}$ & $\frac{1}{4}$ & 10 & 1 & -1 & $-\frac{1}{2}$ \\
$(\mathbb{1}, \mathbb{1}, \mathbb{1}, \mathbb{1})$ & 1 & 0 & 0 & $\frac{1}{3}$ & $\frac{1}{2}$ & $-\frac{1}{6}$ & 1 & $-\frac{5}{12}$ & $\frac{1}{4}$ & -10 & 1 & -1 & $\frac{1}{2}$ \\
$(\mathbf{2}, \mathbb{1}, \mathbb{1}, \mathbb{1})$ & 1 & 1 & 0 & $\frac{1}{3}$ & 0 & $\frac{1}{3}$ & -1 & $\frac{1}{12}$ & $\frac{3}{4}$ & 0 & -1 & 1 & 0 \\
$(\mathbb{1}, \mathbb{1}, \mathbb{1}, \mathbb{1})$ & 1 & 1 & 0 & $\frac{2}{3}$ & 0 & $-\frac{2}{3}$ & 0 & $\frac{1}{12}$ & $\frac{3}{4}$ & -10 & -1 & 1 & $\frac{1}{2}$ \\
$(\mathbb{1}, \mathbb{1}, \mathbb{1}, \mathbb{1})$ & 1 & 1 & 0 & 1 & $\frac{1}{2}$ & $-\frac{1}{6}$ & 1 & $\frac{1}{12}$ & $\frac{3}{4}$ & 10 & -1 & 1 & $-\frac{1}{2}$ \\
$(\mathbf{2}, \mathbb{1}, \mathbb{1}, \mathbb{1})$ & 1 & 2 & 0 & $\frac{1}{3}$ & 0 & $\frac{1}{3}$ & 1 & $-\frac{5}{12}$ & $\frac{1}{4}$ & 0 & -1 & -1 & 0 \\
$(\mathbf{2}, \mathbb{1}, \mathbb{1}, \mathbb{1})$ & 1 & 2 & 0 & 1 & 0 & $\frac{1}{3}$ & 1 & $\frac{1}{12}$ & $\frac{3}{4}$ & 0 & 1 & 1 & 0 \\
$(\mathbb{1}, \mathbb{1}, \mathbb{1}, \mathbb{1})$ & 1 & 2 & 0 & $\frac{2}{3}$ & $\frac{1}{2}$ & $-\frac{1}{6}$ & -1 & $-\frac{5}{12}$ & $\frac{1}{4}$ & -10 & -1 & -1 & $\frac{1}{2}$ \\
$(\mathbb{1}, \mathbb{1}, \mathbb{1}, \mathbb{1})$ & 1 & 2 & 0 & $\frac{2}{3}$ & 0 & $-\frac{2}{3}$ & 0 & $\frac{1}{12}$ & $\frac{3}{4}$ & 10 & 1 & 1 & $-\frac{1}{2}$ \\
$(\mathbb{1}, \mathbb{1}, \mathbb{1}, \mathbb{1})$ & 1 & 2 & 0 & $\frac{1}{3}$ & $\frac{1}{2}$ & $-\frac{1}{6}$ & -1 & $\frac{1}{12}$ & $\frac{3}{4}$ & -10 & 1 & 1 & $\frac{1}{2}$ \\
$(\mathbb{1}, \mathbb{1}, \mathbb{1}, \mathbb{1})$ & 1 & 2 & 0 & 1 & 0 & $-\frac{2}{3}$ & 0 & $-\frac{5}{12}$ & $\frac{1}{4}$ & 10 & -1 & -1 & $-\frac{1}{2}$ \\
\hline$(\mathbb{1}, \mathbb{1}, \mathbb{1}, \mathbb{1})$ & 3 & 0 & 0 & 0 & $\frac{1}{2}$ & $\frac{1}{2}$ & 1 & $-\frac{1}{4}$ & $-\frac{1}{4}$ & -10 & -1 & 1 & $\frac{1}{2}$ \\
$(\mathbb{1}, \mathbb{1}, \mathbb{1}, \mathbb{1})$ & 3 & 0 & 0 & 0 & 0 & 0 & 2 & $\frac{1}{4}$ & $\frac{1}{4}$ & 10 & 1 & -1 & $-\frac{1}{2}$ \\
$(\mathbb{1}, \mathbb{1}, \mathbb{1}, \mathbb{1})$ & 3 & 0 & $\frac{1}{3}$ & 0 & $\frac{1}{2}$ & $\frac{1}{2}$ & -1 & $\frac{1}{4}$ & $\frac{1}{4}$ & 10 & 1 & -1 & $-\frac{1}{2}$ \\
$(\mathbb{1}, \mathbb{1}, \mathbb{1}, \mathbb{1})$ & 3 & 0 & $\frac{1}{3}$ & 0 & $-\frac{1}{2}$ & $-\frac{1}{2}$ & 1 & $-\frac{1}{4}$ & $-\frac{1}{4}$ & -10 & -1 & 1 & $\frac{1}{2}$ \\
$(\mathbb{1}, \mathbb{1}, \mathbb{1}, \mathbb{1})$ & 3 & 0 & $\frac{2}{3}$ & 0 & 0 & 0 & -2 & $-\frac{1}{4}$ & $-\frac{1}{4}$ & -10 & -1 & 1 & $\frac{1}{2}$ \\
$(\mathbb{1}, \mathbb{1}, \mathbb{1}, \mathbb{1})$ & 3 & 0 & $\frac{2}{3}$ & 0 & $-\frac{1}{2}$ & $-\frac{1}{2}$ & -1 & $\frac{1}{4}$ & $\frac{1}{4}$ & 10 & 1 & -1 & $-\frac{1}{2}$ \\
$(\mathbb{1}, \mathbb{1}, \mathbb{1}, \mathbb{1})$ & 3 & 0 & 1 & 0 & $\frac{1}{2}$ & $\frac{1}{2}$ & 1 & $-\frac{1}{4}$ & $-\frac{1}{4}$ & -10 & -1 & 1 & $\frac{1}{2}$ \\
$(\mathbb{1}, \mathbb{1}, \mathbb{1}, \mathbb{1})$ & 3 & 0 & 1 & 0 & 0 & 0 & 2 & $\frac{1}{4}$ & $\frac{1}{4}$ & 10 & 1 & -1 & $-\frac{1}{2}$
\end{tabular}

Table 26: Multiplets of $\mathrm{SU}(2)_{L} \times \mathrm{SU}(4) \times\left[\mathrm{SU}(2)^{\prime} \times \mathrm{SU}(4)^{\prime}\right]$ at $\left(n_{2}, n_{2}^{\prime}\right)=(1,0)$ from the twisted sectors. The spectrum at $\left(n_{2}, n_{2}^{\prime}\right)=(1,1)$ is an exact copy. All states are exotics, they are either weak doublets or are charged under hypercharge with generator $t_{Y}$.

where $\alpha_{\mathcal{S}}^{\prime}$ and $\mathbf{r}_{\mathcal{S}}^{\prime}$ can be found in Tables 15,18 and 18 for the vacua $\mathcal{S}=\mathcal{S}_{0}, \mathcal{S}_{1}, \mathcal{S}_{2}$, respectively. The phases $\eta_{\mathcal{S}}(\phi)$ for all fields $\phi$ at the GUT fixed point $\left(n_{2}, n_{2}^{\prime}\right)=(0,0)$ are listed in Tables 31 and 32. These tables confirm that the given symmetries are unbroken in the above vacua.

Furthermore, from the tables one can infer if a coupling of interest can be present in the superpotential or not. This follows from comparison of the total phase with the vacuum phase with respect to the unbroken symmetries,

$$
\eta_{\mathcal{S}_{0}}\left(\mathcal{K}_{\text {vac }}\right)= \begin{cases}\frac{5}{6}, & \text { for }\left[\tilde{\mathbb{Z}}_{24}\right]_{R}, \\ \frac{1}{3}, & \text { for }\left[\mathbb{Z}_{6}\right]_{R}, \\ 0, & \text { else },\end{cases}
$$




\begin{tabular}{c|c||c||c|c||c|c|c|c|c|c|c|c}
\multicolumn{1}{c|}{ Bulk } & $n_{2}=1$ & $H_{L}$ & $H_{R}$ & $t_{1}$ & $t_{2}$ & $t_{3}$ & $t_{4}$ & $t_{5}$ & $t_{6}^{1}$ & $t_{7}$ & $t_{8}$ \\
\hline \hline$(\mathbf{2 0}, \mathbb{1}, \mathbb{1})$ & & $(\mathbf{2}, \mathbf{6}, \mathbb{1}, \mathbb{1})$ & - & + & $-\frac{1}{2}$ & $\frac{1}{2}$ & 0 & 0 & 0 & 0 & 0 & 0 \\
& & $(\mathbb{1}, \mathbf{4}, \mathbb{1}, \mathbb{1})$ & + & - & $-\frac{1}{2}$ & $\frac{1}{2}$ & 0 & 0 & 0 & -15 & 0 & 0 \\
& & $(\mathbb{1}, \overline{\mathbf{4}}, \mathbb{1}, \mathbb{1})$ & + & - & $-\frac{1}{2}$ & $\frac{1}{2}$ & 0 & 0 & 0 & 15 & 0 & 0 \\
\hline$(\mathbb{1}, \mathbb{1}, \mathbf{8})$ & & $(\mathbb{1}, \mathbb{1}, \mathbb{1}, \overline{\mathbf{4}})$ & + & - & 0 & 0 & 0 & -1 & 0 & 0 & 0 & 1 \\
& & $(\mathbb{1}, \mathbb{1}, \mathbb{1}, \mathbf{4})$ & - & + & 0 & 0 & 0 & -1 & 0 & 0 & 0 & -1 \\
\hline$\left(\mathbb{1}, \mathbb{1}, \boldsymbol{8}_{s}\right)$ & & $(\mathbb{1}, \mathbb{1}, \mathbb{1}, \mathbf{4})$ & - & + & 0 & 0 & 0 & $\frac{1}{2}$ & $\frac{3}{2}$ & 0 & 0 & 1 \\
& & $(\mathbb{1}, \mathbb{1}, \mathbb{1}, \overline{\mathbf{4}})$ & + & - & 0 & 0 & 0 & $\frac{1}{2}$ & $\frac{3}{2}$ & 0 & 0 & -1 \\
\hline$\left(\mathbb{1}, \mathbb{1}, \boldsymbol{8}_{c}\right)$ & & $(\mathbb{1}, \mathbb{1}, \mathbb{1}, \mathbf{6})$ & - & + & 0 & 0 & 0 & $\frac{1}{2}$ & $-\frac{3}{2}$ & 0 & 0 & 0 \\
& & $(\mathbb{1}, \mathbb{1}, \mathbb{1}, \mathbb{1})$ & + & - & 0 & 0 & 0 & $\frac{1}{2}$ & $-\frac{3}{2}$ & 0 & 0 & 2 \\
& & $(\mathbb{1}, \mathbb{1}, \mathbb{1}, \mathbb{1})$ & + & - & 0 & 0 & 0 & $\frac{1}{2}$ & $-\frac{3}{2}$ & 0 & 0 & -2 \\
\hline$(\mathbb{1}, \mathbb{1}, \mathbb{1})$ & $U_{1}$ & $(\mathbb{1}, \mathbb{1}, \mathbb{1}, \mathbb{1})$ & - & + & $\frac{1}{2}$ & $\frac{1}{2}$ & 3 & 0 & 0 & 0 & 0 & 0 \\
$(\mathbb{1}, \mathbb{1}, \mathbb{1})$ & $U_{2}$ & $(\mathbb{1}, \mathbb{1}, \mathbb{1}, \mathbb{1})$ & - & + & $\frac{1}{2}$ & $\frac{1}{2}$ & -3 & 0 & 0 & 0 & 0 & 0 \\
$(\mathbb{1}, \mathbb{1}, \mathbb{1})$ & $U_{3}$ & $(\mathbb{1}, \mathbb{1}, \mathbb{1}, \mathbb{1})$ & - & + & 1 & -1 & 0 & 0 & 0 & 0 & 0 & 0 \\
$(\mathbb{1}, \mathbb{1}, \mathbb{1})$ & $U_{4}$ & $(\mathbb{1}, \mathbb{1}, \mathbb{1}, \mathbb{1})$ & - & + & -1 & -1 & 0 & 0 & 0 & 0 & 0 & 0
\end{tabular}

Table 27: Chiral projection of bulk hypermultiplets multiplets from the untwisted sector to the fixed points $n_{2}=1$. The representations are given with respect to $\mathrm{SU}(6) \times[\mathrm{SU}(3) \times \mathrm{SO}(8)]$ and $\mathrm{SU}(2) \times \mathrm{SU}(4) \times\left[\mathrm{SU}(2)^{\prime} \times \mathrm{SU}(4)^{\prime}\right]$ in the bulk and at $n_{2}=1$, respectively.

$$
\eta_{\mathcal{S}_{1}}\left(\mathcal{K}_{\mathrm{vac}}\right)=\left\{\begin{array}{ll}
\frac{1}{3}, & \text { for }\left[\mathbb{Z}_{6}\right]_{R}, \\
0, & \text { else },
\end{array} \quad \eta_{\mathcal{S}_{2}}\left(\mathcal{K}_{\mathrm{vac}}\right)= \begin{cases}\frac{1}{2}, & \text { for }\left[\tilde{\mathbb{Z}}_{4}\right]_{R}, \\
0, & \text { else. }\end{cases}\right.
$$

Couplings may be allowed if the sums of the phases add to the above vacuum values, up to integers, and for all unbroken symmetries. Otherwise, they are forbidden to arbitrary order in the singlets.

Note that the breaking of symmetries is insensitive to overall minus signs. This implies that singlets $s$ and $s^{-1}$, in the sense of Section 4.6, yield the same unbroken symmetry. In consequence, it is possible that a coupling is allowed from the viewpoint of the unbroken symmetries, but always involves singlets with negative powers, and is therefore forbidden for the description of physical interactions. As an example, consider the dimension-five proton decay operator from Equation (329) in the vacuum $\mathcal{S}_{0}$,

$$
\eta_{\mathcal{S}_{0}}\left(10_{(1)} 10_{(1)} 10_{(1)} \overline{\mathbf{5}}_{(1)}\right)=\eta_{\mathcal{S}_{0}}\left(\mathcal{K}_{\text {vac }}\right) .
$$

This term is consistent with the unbroken symmetries of the vacuum. However, an example for a corresponding coupling reads

$$
X_{0}^{-1}\left(\bar{X}_{0}^{c}\right)^{2} S_{2} S_{5} \mathbf{1 0}_{(1)} \mathbf{1 0}_{(1)} \mathbf{1 0}_{(1)} \overline{\mathbf{5}}_{(1)},
$$

and in fact it is impossible to find monomials with only positive exponents. Since the symmetries do not know about the projection rule (323) for physical couplings, they can only state necessary conditions for their existence. 


\begin{tabular}{c||c|c||c|c||c|c||c|c|c|c|c|c|c|c} 
Bulk & \multicolumn{2}{|c|}{$n_{2}=1$} & $n_{3}$ & $\gamma_{3}^{\prime}$ & $H_{L}$ & $H_{R}$ & $t_{1}$ & $t_{2}$ & $t_{3}$ & $t_{4}$ & $t_{5}$ & $t_{6}^{1}$ & $t_{7}$ & $t_{8}$ \\
\hline \hline$(\mathbb{1}, \mathbb{1}, \mathbb{1})$ & $(\mathbb{1}, \mathbb{1}, \mathbb{1}, \mathbb{1})$ & $Y_{0}^{*}$ & 0 & $\frac{2}{3}$ &,+-+ &,,-+- & 0 & $\frac{2}{3}$ & 0 & $\frac{2}{3}$ & 0 & 0 & 0 & 0 \\
\hline$(\mathbb{1}, \mathbb{1}, \mathbb{1})$ & $(\mathbb{1}, \mathbb{1}, \mathbb{1}, \mathbb{1})$ & $\bar{Y}_{0}^{*}$ & 0 & $\frac{2}{3}$ &,,-+- &,,+-+ & 0 & $\frac{2}{3}$ & 0 & $\frac{2}{3}$ & 0 & 0 & 0 & 0 \\
\hline \hline$(\mathbb{1}, \mathbb{1}, \mathbb{1})$ & $(\mathbb{1}, \mathbb{1}, \mathbb{1}, \mathbb{1})$ & $Y_{1}^{*}$ & 1 & $\frac{2}{3}$ &,+-+ &,,-+- & $-\frac{1}{2}$ & $\frac{1}{6}$ & 1 & $-\frac{1}{3}$ & -1 & 0 & 0 & 0 \\
\hline$(\mathbb{1}, \mathbb{1}, \mathbb{1})$ & $(\mathbb{1}, \mathbb{1}, \mathbb{1}, \mathbb{1})$ & $\bar{Y}_{1}^{*}$ & 1 & $\frac{2}{3}$ &,,-+- &,,+-+ & $-\frac{1}{2}$ & $\frac{1}{6}$ & 1 & $-\frac{1}{3}$ & -1 & 0 & 0 & 0 \\
\hline \hline$(\mathbb{1}, \mathbb{1}, \mathbb{1})$ & $(\mathbb{1}, \mathbb{1}, \mathbb{1}, \mathbb{1})$ & $Y_{2}^{*}$ & 2 & $\frac{2}{3}$ &,,-+- &,,+-+ & $-\frac{1}{2}$ & $\frac{1}{6}$ & -1 & $-\frac{1}{3}$ & 1 & 0 & 0 & 0 \\
\hline$(\mathbb{1}, \mathbb{1}, \mathbb{1})$ & $(\mathbb{1}, \mathbb{1}, \mathbb{1}, \mathbb{1})$ & $\bar{Y}_{2}^{*}$ & 2 & $\frac{2}{3}$ &,+-+ &,,-+- & $-\frac{1}{2}$ & $\frac{1}{6}$ & -1 & $-\frac{1}{3}$ & 1 & 0 & 0 & 0
\end{tabular}

Table 28: Projection of bulk hypermultiplets with oscillator numbers from twisted sectors $T_{2}$ and $T_{4}$ to the fixed points $n_{2}=1$. The singlets $Y_{n_{3}}^{*}$ have $\tilde{\mathbf{N}}=(0,1,0,0), \tilde{\mathbf{N}}^{*}=(0,0,0,0)$, the singlets $\bar{Y}_{n_{3}}^{*}$ have $\tilde{\mathbf{N}}=(0,0,0,0), \tilde{\mathbf{N}}^{*}=(1,0,0,0)$. The three parities for the chiral multiplet components $H_{L}, H_{R}$ correspond to $\gamma=0, \frac{1}{2}, 1$. The localization in the $\mathrm{G}_{2}$-plane is given by $n_{6}=0$ for $\gamma=0$, and $n_{6}=2$ otherwise.

\begin{tabular}{|c|c|c|c|c|c|c|c|c|c|c|c|c|c|}
\hline \multirow[b]{2}{*}{ Multiplet } & \multirow[b]{2}{*}{$k$} & \multirow[b]{2}{*}{$n_{3}$} & \multicolumn{4}{|c|}{ False vacuum } & \multicolumn{2}{|c|}{ Vacuum $\mathcal{S}_{0}$} & \multicolumn{3}{|c|}{ Vacuum $\mathcal{S}_{1}$} & \multicolumn{2}{|c|}{ Vacuum $\mathcal{S}_{2}$} \\
\hline & & & $R^{1}$ & $\overline{R^{2}}$ & $R^{3}$ & $t_{X}$ & {$\left[\tilde{\mathbb{Z}}_{24}\right]_{R}$} & {$\left[\mathbb{Z}_{6}\right]_{R}$} & {$\left[\mathbb{Z}_{6}\right]_{R}$} & {$\left[\tilde{\mathbb{Z}}_{2}\right]_{R}$} & $\mathbb{Z}_{2}$ & {$\left[\tilde{\mathbb{Z}}_{4}\right]_{R}$} & {$\left[\mathbb{Z}_{2}\right]_{R}$} \\
\hline$(10, \mathbb{1}, \mathbb{1})$ & 0 & 0 & 0 & -1 & 0 & $\frac{1}{5}$ & $\frac{1}{60}$ & $\frac{2}{3}$ & $\frac{2}{3}$ & $\frac{1}{2}$ & 0 & $\frac{3}{20}$ & 0 \\
\hline$(10, \mathbb{1}, \mathbb{1})$ & 0 & 0 & -1 & 0 & 0 & $\frac{1}{5}$ & $\frac{1}{60}$ & $\frac{2}{3}$ & $\frac{2}{3}$ & $\frac{1}{2}$ & 0 & $\frac{3}{20}$ & 0 \\
\hline$(5, \mathbb{1}, \mathbb{1})$ & 0 & 0 & 0 & 0 & -1 & $-\frac{2}{5}$ & $\frac{4}{5}$ & 0 & 0 & 0 & 0 & $\frac{1}{5}$ & 0 \\
\hline$(\overline{\mathbf{5}}, \mathbb{1}, \mathbb{1})$ & 0 & 0 & 0 & 0 & -1 & $\frac{2}{5}$ & $\frac{1}{5}$ & 0 & 0 & 0 & 0 & $\frac{4}{5}$ & 0 \\
\hline$(\mathbb{1}, \mathbb{1}, \mathbf{8})$ & 0 & 0 & 0 & -1 & 0 & $\frac{1}{2}$ & $\frac{23}{24}$ & $\frac{1}{3}$ & $\frac{5}{6}$ & $\frac{3}{4}$ & $\frac{1}{2}$ & $\frac{5}{8}$ & 0 \\
\hline$\left(\mathbb{1}, \mathbb{1}, \boldsymbol{8}_{c}\right)$ & 0 & 0 & -1 & 0 & 0 & 0 & $\frac{13}{16}$ & 0 & 0 & $\frac{1}{2}$ & $\frac{1}{2}$ & $\frac{3}{4}$ & $\frac{1}{2}$ \\
\hline$\left(\mathbb{1}, \mathbb{1}, \mathbf{8}_{s}\right)$ & 0 & 0 & -1 & 0 & 0 & $\frac{1}{2}$ & $\frac{13}{16}$ & 0 & $\frac{1}{2}$ & $\frac{1}{4}$ & 0 & $\frac{7}{8}$ & $\frac{1}{2}$ \\
\hline$(10, \mathbb{1}, \mathbb{1})$ & 1 & 0 & $-\frac{1}{6}$ & $-\frac{1}{3}$ & $-\frac{1}{2}$ & $\frac{1}{5}$ & $\frac{1}{60}$ & $\frac{2}{3}$ & $\frac{2}{3}$ & $\frac{1}{2}$ & 0 & $\frac{3}{20}$ & 0 \\
\hline$(\overline{\mathbf{5}}, \mathbb{1}, \mathbb{1})$ & 1 & 0 & $-\frac{1}{6}$ & $-\frac{1}{3}$ & $-\frac{1}{2}$ & $-\frac{3}{5}$ & $\frac{47}{60}$ & $\frac{1}{3}$ & $\frac{1}{3}$ & $\frac{1}{2}$ & 0 & $\frac{1}{20}$ & 0 \\
\hline$(\mathbb{1}, \overline{\mathbf{3}}, \mathbb{1})$ & 1 & 1 & $\frac{5}{6}$ & $-\frac{1}{3}$ & $-\frac{1}{2}$ & $\frac{1}{3}$ & $\frac{1}{4}$ & 0 & $\frac{2}{3}$ & $\frac{1}{2}$ & 0 & $\frac{7}{12}$ & 0 \\
\hline$\left(\mathbb{1}, \mathbb{1}, \boldsymbol{8}_{c}\right)$ & 1 & 1 & $-\frac{1}{6}$ & $-\frac{1}{3}$ & $-\frac{1}{2}$ & 0 & $\frac{5}{48}$ & 0 & 0 & $\frac{1}{2}$ & $\frac{1}{2}$ & $\frac{3}{4}$ & $\frac{1}{2}$ \\
\hline$(\mathbb{1}, \mathbf{3}, \mathbb{1})$ & 1 & 2 & $-\frac{1}{6}$ & $-\frac{1}{3}$ & $-\frac{1}{2}$ & $-\frac{1}{3}$ & $\frac{11}{12}$ & 0 & 0 & $\frac{1}{2}$ & 0 & $\frac{11}{12}$ & 0 \\
\hline$(5, \mathbb{1}, \mathbb{1})$ & 2 & 0 & $-\frac{1}{3}$ & $-\frac{2}{3}$ & 0 & $-\frac{2}{5}$ & $\frac{4}{5}$ & 0 & 0 & 0 & 0 & $\frac{1}{5}$ & 0 \\
\hline$(\overline{\mathbf{5}}, \mathbb{1}, \mathbb{1})$ & 2 & 0 & $-\frac{1}{3}$ & $-\frac{2}{3}$ & 0 & $\frac{2}{5}$ & $\frac{1}{30}$ & $\frac{1}{3}$ & $\frac{1}{3}$ & 0 & 0 & $\frac{3}{10}$ & 0 \\
\hline$(\mathbb{1}, \mathbf{3}, \mathbb{1})$ & 2 & 0 & $-\frac{1}{3}$ & $-\frac{2}{3}$ & 0 & $\frac{2}{3}$ & $\frac{23}{24}$ & $\frac{1}{3}$ & 0 & 0 & 0 & $\frac{2}{3}$ & 0 \\
\hline$(\mathbb{1}, \overline{\mathbf{3}}, \mathbb{1})$ & 2 & 0 & $-\frac{1}{3}$ & $-\frac{2}{3}$ & 0 & $\frac{1}{3}$ & $\frac{23}{24}$ & $\frac{1}{3}$ & $\frac{2}{3}$ & $\frac{1}{2}$ & 0 & $\frac{7}{12}$ & 0 \\
\hline$(\mathbb{1}, \mathbb{1}, \mathbf{8})$ & 2 & 0 & $-\frac{1}{3}$ & $-\frac{2}{3}$ & 0 & $\frac{1}{2}$ & $\frac{23}{24}$ & $\frac{1}{3}$ & $\frac{5}{6}$ & $\frac{3}{4}$ & $\frac{1}{2}$ & $\frac{5}{8}$ & 0 \\
\hline$(5, \mathbb{1}, \mathbb{1})$ & 2 & 1 & $-\frac{1}{3}$ & $-\frac{2}{3}$ & 0 & $-\frac{2}{5}$ & $\frac{1}{120}$ & 0 & 0 & 0 & 0 & $\frac{7}{10}$ & 0 \\
\hline$(\overline{\mathbf{5}}, \mathbb{1}, \mathbb{1})$ & 2 & 1 & $-\frac{1}{3}$ & $-\frac{2}{3}$ & 0 & $\frac{2}{5}$ & $\frac{109}{120}$ & $\frac{1}{3}$ & 0 & 0 & 0 & $\frac{4}{5}$ & 0 \\
\hline$(\mathbb{1}, \mathbf{3}, \mathbb{1})$ & 2 & 1 & $-\frac{1}{3}$ & $-\frac{2}{3}$ & 0 & $-\frac{1}{3}$ & $\frac{11}{12}$ & $\frac{1}{3}$ & 0 & $\frac{1}{2}$ & 0 & $\frac{11}{12}$ & 0 \\
\hline
\end{tabular}


Table 31 - continued from previous page

\begin{tabular}{|c|c|c|c|c|c|c|c|c|c|c|c|c|c|}
\hline \multirow[b]{2}{*}{ Multiplet } & \multirow[b]{2}{*}{$k$} & \multirow[b]{2}{*}{$n_{3}$} & \multicolumn{4}{|c|}{ False vacuum } & \multicolumn{2}{|c|}{ Vacuum $\mathcal{S}_{0}$} & \multicolumn{3}{|c|}{ Vacuum $\mathcal{S}_{1}$} & \multicolumn{2}{|c|}{ Vacuum $\mathcal{S}_{2}$} \\
\hline & & & $R^{1}$ & $R^{2}$ & $R^{3}$ & $t_{X}$ & {$\left[\tilde{\mathbb{Z}}_{24}\right]_{R}$} & {$\left[\mathbb{Z}_{6}\right]_{R}$} & {$\left[\mathbb{Z}_{6}\right]_{R}$} & {$\left[\tilde{\mathbb{Z}}_{2}\right]_{R}$} & $\mathbb{Z}_{2}$ & {$\left[\tilde{\mathbb{Z}}_{4}\right]_{R}$} & {$\left[\mathbb{Z}_{2}\right]_{R}$} \\
\hline$(\mathbb{1}, \overline{\mathbf{3}}, \mathbb{1})$ & 2 & 1 & $-\frac{1}{3}$ & $-\frac{2}{3}$ & 0 & $-\frac{2}{3}$ & $\frac{23}{24}$ & $\frac{1}{3}$ & $\frac{1}{3}$ & 0 & 0 & $\frac{1}{3}$ & 0 \\
\hline$\left(\mathbb{1}, \mathbb{1}, \mathbf{8}_{s}\right)$ & 2 & 1 & $-\frac{1}{3}$ & $-\frac{2}{3}$ & 0 & $-\frac{1}{2}$ & $\frac{15}{16}$ & $\frac{1}{3}$ & $\frac{1}{6}$ & $\frac{3}{4}$ & 0 & $\frac{5}{8}$ & $\frac{1}{2}$ \\
\hline$(5, \mathbb{1}, \mathbb{1})$ & 2 & 2 & $-\frac{1}{3}$ & $-\frac{2}{3}$ & 0 & $\frac{3}{5}$ & $\frac{101}{120}$ & 0 & 0 & $\frac{1}{2}$ & 0 & $\frac{19}{20}$ & 0 \\
\hline$(\overline{\mathbf{5}}, \mathbb{1}, \mathbb{1})$ & 2 & 2 & $-\frac{1}{3}$ & $-\frac{2}{3}$ & 0 & $-\frac{3}{5}$ & $\frac{109}{120}$ & $\frac{1}{3}$ & $\frac{2}{3}$ & $\frac{1}{2}$ & 0 & $\frac{11}{20}$ & 0 \\
\hline$(\mathbb{1}, \mathbf{3}, \mathbb{1})$ & 2 & 2 & $-\frac{1}{3}$ & $-\frac{2}{3}$ & 0 & $-\frac{1}{3}$ & $\frac{1}{8}$ & $\frac{1}{3}$ & 0 & $\frac{1}{2}$ & 0 & $\frac{5}{12}$ & 0 \\
\hline$(\mathbb{1}, \overline{\mathbf{3}}, \mathbb{1})$ & 2 & 2 & $-\frac{1}{3}$ & $-\frac{2}{3}$ & 0 & $\frac{1}{3}$ & $\frac{1}{12}$ & $\frac{1}{3}$ & 0 & $\frac{1}{2}$ & 0 & $\frac{1}{12}$ & 0 \\
\hline$\left(\mathbb{1}, \mathbb{1}, \mathbf{8}_{c}\right)$ & 2 & 2 & $-\frac{1}{3}$ & $-\frac{2}{3}$ & 0 & 0 & $\frac{5}{48}$ & $\frac{1}{3}$ & 0 & $\frac{1}{2}$ & $\frac{1}{2}$ & $\frac{3}{4}$ & $\frac{1}{2}$ \\
\hline$(\mathbb{1}, \mathbf{3}, \mathbb{1})$ & 3 & 0 & $-\frac{1}{2}$ & 0 & $-\frac{1}{2}$ & $-\frac{1}{3}$ & $\frac{1}{24}$ & 0 & $\frac{1}{3}$ & $\frac{1}{2}$ & 0 & $\frac{5}{12}$ & 0 \\
\hline$(\mathbb{1}, \overline{\mathbf{3}}, \mathbb{1})$ & 3 & 0 & $-\frac{1}{2}$ & 0 & $-\frac{1}{2}$ & $\frac{1}{3}$ & $\frac{19}{24}$ & 0 & 0 & $\frac{1}{2}$ & 0 & $\frac{1}{12}$ & 0 \\
\hline$(5, \mathbb{1}, \mathbb{1})$ & 4 & 0 & $-\frac{2}{3}$ & $-\frac{1}{3}$ & 0 & $-\frac{2}{5}$ & $\frac{4}{5}$ & 0 & 0 & 0 & 0 & $\frac{1}{5}$ & 0 \\
\hline$(\overline{\mathbf{5}}, \mathbb{1}, \mathbb{1})$ & 4 & 0 & $-\frac{2}{3}$ & $-\frac{1}{3}$ & 0 & $\frac{2}{5}$ & $\frac{1}{30}$ & $\frac{1}{3}$ & $\frac{1}{3}$ & 0 & 0 & $\frac{3}{10}$ & 0 \\
\hline$(\mathbb{1}, \mathbf{3}, \mathbb{1})$ & 4 & 0 & $-\frac{2}{3}$ & $-\frac{1}{3}$ & 0 & $-\frac{1}{3}$ & $\frac{7}{8}$ & 0 & $\frac{2}{3}$ & $\frac{1}{2}$ & 0 & $\frac{11}{12}$ & 0 \\
\hline$(\mathbb{1}, \overline{\mathbf{3}}, \mathbb{1})$ & 4 & 0 & $-\frac{2}{3}$ & $-\frac{1}{3}$ & 0 & $-\frac{2}{3}$ & $\frac{7}{8}$ & 0 & $\frac{1}{3}$ & 0 & 0 & $\frac{5}{6}$ & 0 \\
\hline$(\mathbb{1}, \mathbb{1}, \mathbf{8})$ & 4 & 0 & $-\frac{2}{3}$ & $-\frac{1}{3}$ & 0 & $-\frac{1}{2}$ & $\frac{7}{8}$ & 0 & $\frac{1}{2}$ & $\frac{1}{4}$ & $\frac{1}{2}$ & $\frac{7}{8}$ & 0 \\
\hline$(5, \mathbb{1}, \mathbb{1})$ & 4 & 1 & $-\frac{2}{3}$ & $-\frac{1}{3}$ & 0 & $-\frac{2}{5}$ & $\frac{37}{40}$ & 0 & $\frac{1}{3}$ & 0 & 0 & $\frac{7}{10}$ & 0 \\
\hline$(\overline{\mathbf{5}}, \mathbb{1}, \mathbb{1})$ & 4 & 1 & $-\frac{2}{3}$ & $-\frac{1}{3}$ & 0 & $\frac{2}{5}$ & $\frac{33}{40}$ & $\frac{1}{3}$ & $\frac{1}{3}$ & 0 & 0 & $\frac{4}{5}$ & 0 \\
\hline$(\mathbb{1}, \mathbf{3}, \mathbb{1})$ & 4 & 1 & $-\frac{2}{3}$ & $-\frac{1}{3}$ & 0 & $\frac{2}{3}$ & $\frac{7}{8}$ & 0 & 0 & 0 & 0 & $\frac{1}{6}$ & 0 \\
\hline$(\mathbb{1}, \overline{\mathbf{3}}, \mathbb{1})$ & 4 & 1 & $-\frac{2}{3}$ & $-\frac{1}{3}$ & 0 & $\frac{1}{3}$ & $\frac{11}{12}$ & 0 & $\frac{1}{3}$ & $\frac{1}{2}$ & 0 & $\frac{7}{12}$ & 0 \\
\hline$\left(\mathbb{1}, \mathbb{1}, \boldsymbol{8}_{s}\right)$ & 4 & 1 & $-\frac{2}{3}$ & $-\frac{1}{3}$ & 0 & $\frac{1}{2}$ & $\frac{43}{48}$ & 0 & $\frac{1}{6}$ & $\frac{1}{4}$ & 0 & $\frac{7}{8}$ & $\frac{1}{2}$ \\
\hline$(5, \mathbb{1}, \mathbb{1})$ & 4 & 2 & $-\frac{2}{3}$ & $-\frac{1}{3}$ & 0 & $\frac{3}{5}$ & $\frac{37}{40}$ & 0 & $\frac{2}{3}$ & $\frac{1}{2}$ & 0 & $\frac{19}{20}$ & 0 \\
\hline$(\overline{\mathbf{5}}, \mathbb{1}, \mathbb{1})$ & 4 & 2 & $-\frac{2}{3}$ & $-\frac{1}{3}$ & 0 & $-\frac{3}{5}$ & $\frac{119}{120}$ & $\frac{1}{3}$ & $\frac{1}{3}$ & $\frac{1}{2}$ & 0 & $\frac{11}{20}$ & 0 \\
\hline$(\mathbb{1}, \mathbf{3}, \mathbb{1})$ & 4 & 2 & $-\frac{2}{3}$ & $-\frac{1}{3}$ & 0 & $-\frac{1}{3}$ & $\frac{3}{4}$ & 0 & $\frac{1}{3}$ & $\frac{1}{2}$ & 0 & $\frac{5}{12}$ & 0 \\
\hline$(\mathbb{1}, \overline{\mathbf{3}}, \mathbb{1})$ & 4 & 2 & $-\frac{2}{3}$ & $-\frac{1}{3}$ & 0 & $\frac{1}{3}$ & $\frac{17}{24}$ & 0 & $\frac{1}{3}$ & $\frac{1}{2}$ & 0 & $\frac{1}{12}$ & 0 \\
\hline$\left(\mathbb{1}, \mathbb{1}, \boldsymbol{8}_{c}\right)$ & 4 & 2 & $-\frac{2}{3}$ & $-\frac{1}{3}$ & 0 & 0 & $\frac{35}{48}$ & 0 & $\frac{1}{3}$ & $\frac{1}{2}$ & $\frac{1}{2}$ & $\frac{3}{4}$ & $\frac{1}{2}$ \\
\hline
\end{tabular}

Table 31: Discrete transformation phases $\eta_{\mathcal{S}}(\phi)$ for non-singlets $\phi$ at $\left(n_{2}, n_{2}^{\prime}\right)=(0,0)$. The tilde in $\tilde{\mathbb{Z}}_{N}$ denotes that the $N$-th application of the generator associated with the symmetry yields not necessarily an integer, but a phase which can be expressed by other generators. Note that the vacua $\mathcal{S}_{1}, \mathcal{S}_{2}$ additionally have an unbroken symmetry whose generator is proportional to $\frac{1}{2} t_{X}$, for $\mathcal{S}_{0}$ this holds up to $t_{5}$. $\mathcal{S}_{0}$ has $\mathrm{U}(1)_{5}$ as an unbroken continuous symmetry. 


\begin{tabular}{|c|c|c|c|c|c|c|c|c|c|c|c|c|c|}
\hline \multirow[b]{2}{*}{ Singlet } & \multirow[b]{2}{*}{$k$} & \multirow[b]{2}{*}{$n_{3}$} & \multicolumn{4}{|c|}{ False vacuum } & \multicolumn{2}{|c|}{ Vacuum $\mathcal{S}_{0}$} & \multicolumn{3}{|c|}{ Vacuum $\mathcal{S}_{1}$} & \multicolumn{2}{|c|}{ Vacuum $\mathcal{S}_{2}$} \\
\hline & & & $R^{1}$ & $R^{2}$ & $R^{3}$ & $t_{X}$ & {$\left[\tilde{\mathbb{Z}}_{24}\right]_{R}$} & {$\left[\mathbb{Z}_{6}\right]_{R}$} & {$\left[\mathbb{Z}_{6}\right]_{R}$} & {$\left[\tilde{\mathbb{Z}}_{2}\right]_{R}$} & $\mathbb{Z}_{2}$ & {$\left[\tilde{\mathbb{Z}}_{4}\right]_{R}$} & {$\left[\mathbb{Z}_{2}\right]_{R}$} \\
\hline$U_{1}^{c}$ & 0 & 0 & 0 & -1 & 0 & 0 & $\frac{1}{3}$ & $\frac{1}{3}$ & $\frac{1}{3}$ & 0 & 0 & 0 & 0 \\
\hline$U_{2}$ & 0 & 0 & -1 & 0 & 0 & 2 & 0 & 0 & 0 & 0 & 0 & 0 & 0 \\
\hline$U_{3}$ & 0 & 0 & -1 & 0 & 0 & 0 & $\frac{2}{3}$ & $\frac{2}{3}$ & $\frac{2}{3}$ & 0 & 0 & 0 & 0 \\
\hline$U_{4}$ & 0 & 0 & -1 & 0 & 0 & -2 & 0 & 0 & 0 & 0 & 0 & 0 & 0 \\
\hline$N_{(1)}^{c}$ & 1 & 0 & $-\frac{1}{6}$ & $-\frac{1}{3}$ & $-\frac{1}{2}$ & 1 & $\frac{1}{4}$ & 0 & 0 & $\frac{1}{2}$ & 0 & $\frac{1}{4}$ & 0 \\
\hline$S_{1}$ & 1 & 0 & $\frac{5}{6}$ & $-\frac{1}{3}$ & $-\frac{1}{2}$ & -1 & $\frac{1}{4}$ & 0 & 0 & $\frac{1}{2}$ & 0 & $\frac{1}{4}$ & 0 \\
\hline$S_{2}$ & 1 & 0 & $\frac{5}{6}$ & $-\frac{1}{3}$ & $-\frac{1}{2}$ & 0 & 0 & 0 & 0 & 0 & 0 & 0 & 0 \\
\hline$S_{3}$ & 1 & 0 & $-\frac{1}{6}$ & $\frac{2}{3}$ & $-\frac{1}{2}$ & 1 & $\frac{11}{12}$ & $\frac{2}{3}$ & $\frac{2}{3}$ & $\frac{1}{2}$ & 0 & $\frac{1}{4}$ & 0 \\
\hline$S_{4}$ & 1 & 0 & $\frac{11}{6}$ & $-\frac{1}{3}$ & $-\frac{1}{2}$ & 1 & $\frac{1}{4}$ & 0 & 0 & $\frac{1}{2}$ & 0 & $\frac{1}{4}$ & 0 \\
\hline$S_{5}$ & 1 & 0 & $-\frac{1}{6}$ & $\frac{2}{3}$ & $-\frac{1}{2}$ & 0 & 0 & 0 & 0 & 0 & 0 & 0 & 0 \\
\hline$S_{6}$ & 1 & 0 & $\frac{11}{6}$ & $-\frac{1}{3}$ & $-\frac{1}{2}$ & 0 & $\frac{1}{3}$ & $\frac{1}{3}$ & $\frac{1}{3}$ & 0 & 0 & 0 & 0 \\
\hline$S_{7}$ & 1 & 1 & $\begin{array}{l}\frac{5}{6} \\
\end{array}$ & $-\frac{1}{3}$ & $-\frac{1}{2}$ & 0 & $\frac{7}{24}$ & 0 & 0 & 0 & 0 & 0 & 0 \\
\hline$S_{8}$ & 1 & 2 & $-\frac{1}{6}$ & $-\frac{1}{3}$ & $-\frac{1}{2}$ & -1 & $\frac{23}{24}$ & 0 & 0 & $\frac{1}{2}$ & 0 & $\frac{1}{4}$ & 0 \\
\hline$X_{0}$ & 2 & 0 & $-\frac{1}{3}$ & $-\frac{2}{3}$ & 0 & 0 & 0 & 0 & 0 & 0 & 0 & 0 & 0 \\
\hline $\bar{X}_{0}$ & 2 & 0 & $-\frac{1}{3}$ & $-\frac{2}{3}$ & 0 & 0 & $\frac{5}{6}$ & $\frac{1}{3}$ & $\frac{1}{3}$ & 0 & 0 & $\frac{1}{2}$ & 0 \\
\hline$Y_{0}$ & 2 & 0 & $-\frac{1}{3}$ & $-\frac{2}{3}$ & 0 & 1 & $\frac{3}{4}$ & 0 & 0 & $\frac{1}{2}$ & 0 & $\frac{3}{4}$ & 0 \\
\hline $\bar{Y}_{0}$ & 2 & 0 & $-\frac{1}{3}$ & $-\frac{2}{3}$ & 0 & -1 & $\frac{1}{12}$ & $\frac{1}{3}$ & $\frac{1}{3}$ & $\frac{1}{2}$ & 0 & $\frac{3}{4}$ & 0 \\
\hline$Y_{0}^{*}$ & 2 & 0 & $-\frac{1}{3}$ & $-\frac{5}{3}$ & 0 & 1 & $\frac{11}{12}$ & $\frac{2}{3}$ & $\frac{2}{3}$ & $\frac{1}{2}$ & 0 & $\frac{1}{4}$ & 0 \\
\hline $\bar{Y}_{0}^{*}$ & 2 & 0 & $\frac{2}{3}$ & $-\frac{2}{3}$ & 0 & 1 & $\frac{1}{12}$ & $\frac{1}{3}$ & $\frac{1}{3}$ & $\frac{1}{2}$ & 0 & $\frac{3}{4}$ & 0 \\
\hline$X_{1}$ & 2 & 1 & $-\frac{1}{3}$ & $-\frac{2}{3}$ & 0 & 0 & $\frac{5}{24}$ & 0 & 0 & 0 & 0 & $\frac{1}{2}$ & 0 \\
\hline $\bar{X}_{1}$ & 2 & 1 & $-\frac{1}{3}$ & $-\frac{2}{3}$ & 0 & 0 & $\frac{17}{24}$ & $\frac{1}{3}$ & 0 & 0 & 0 & 0 & 0 \\
\hline$Y_{1}$ & 2 & 1 & $-\frac{1}{3}$ & $-\frac{2}{3}$ & 0 & 1 & $\frac{1}{8}$ & $\frac{1}{3}$ & $\frac{2}{3}$ & $\frac{1}{2}$ & 0 & $\frac{3}{4}$ & 0 \\
\hline $\bar{Y}_{1}$ & 2 & 1 & $-\frac{1}{3}$ & $-\frac{2}{3}$ & 0 & -1 & $\frac{19}{24}$ & 0 & $\frac{1}{3}$ & $\frac{1}{2}$ & 0 & $\frac{3}{4}$ & 0 \\
\hline$Y_{1}^{*}$ & 2 & 1 & $-\frac{1}{3}$ & $-\frac{5}{3}$ & 0 & -1 & $\frac{23}{24}$ & $\frac{2}{3}$ & 0 & $\frac{1}{2}$ & 0 & $\frac{1}{4}$ & 0 \\
\hline $\bar{Y}_{1}^{*}$ & 2 & 1 & $\frac{2}{3}$ & $-\frac{2}{3}$ & 0 & -1 & $\frac{1}{8}$ & $\frac{1}{3}$ & $\frac{2}{3}$ & $\frac{1}{2}$ & 0 & $\frac{3}{4}$ & 0 \\
\hline$X_{2}$ & 2 & 2 & $-\frac{1}{3}$ & $-\frac{2}{3}$ & 0 & 1 & $\frac{1}{24}$ & 0 & 0 & $\frac{1}{2}$ & 0 & $\frac{3}{4}$ & 0 \\
\hline $\bar{X}_{2}$ & 2 & 2 & $-\frac{1}{3}$ & $-\frac{2}{3}$ & 0 & -1 & $\frac{17}{24}$ & $\frac{1}{3}$ & $\frac{2}{3}$ & $\frac{1}{2}$ & 0 & $\frac{3}{4}$ & 0 \\
\hline$Y_{2}$ & 2 & 2 & $-\frac{1}{3}$ & $-\frac{2}{3}$ & 0 & 0 & $\frac{19}{24}$ & $\frac{1}{3}$ & 0 & 0 & 0 & $\frac{1}{2}$ & 0 \\
\hline $\bar{Y}_{2}$ & 2 & 2 & $-\frac{1}{3}$ & $-\frac{2}{3}$ & 0 & 0 & $\frac{23}{24}$ & 0 & $\frac{2}{3}$ & 0 & 0 & 0 & 0 \\
\hline$X_{2}^{*}$ & 2 & 2 & $-\frac{1}{3}$ & $-\frac{5}{3}$ & 0 & 0 & $\frac{1}{8}$ & $\frac{2}{3}$ & $\frac{1}{3}$ & 0 & 0 & $\frac{1}{2}$ & 0 \\
\hline $\bar{X}_{2}^{*}$ & 2 & 2 & $\frac{2}{3}$ & $-\frac{2}{3}$ & 0 & 0 & $\frac{7}{24}$ & $\frac{1}{3}$ & 0 & 0 & 0 & 0 & 0 \\
\hline$X_{0}^{c}$ & 4 & 0 & $-\frac{2}{3}$ & $-\frac{1}{3}$ & 0 & 0 & $\frac{5}{6}$ & $\frac{1}{3}$ & $\frac{1}{3}$ & 0 & 0 & $\frac{1}{2}$ & 0 \\
\hline $\bar{X}_{0}^{c}$ & 4 & 0 & $-\frac{2}{3}$ & $-\frac{1}{3}$ & 0 & 0 & 0 & 0 & 0 & 0 & 0 & 0 & 0 \\
\hline
\end{tabular}

Table 32 - continued on next page 
Table 32 - continued from previous page

\begin{tabular}{|c|c|c|c|c|c|c|c|c|c|c|c|c|c|}
\hline \multirow[b]{2}{*}{ Singlet } & \multirow[b]{2}{*}{$k$} & \multirow[b]{2}{*}{$n_{3}$} & \multicolumn{4}{|c|}{ False vacuum } & \multicolumn{2}{|c|}{ Vacuum $\mathcal{S}_{0}$} & \multicolumn{3}{|c|}{ Vacuum $\mathcal{S}_{1}$} & \multicolumn{2}{|c|}{ Vacuum $\mathcal{S}_{2}$} \\
\hline & & & $R^{1}$ & $R^{2}$ & $R^{3}$ & $t_{X}$ & {$\left[\tilde{\mathbb{Z}}_{24}\right]_{R}$} & {$\left[\mathbb{Z}_{6}\right]_{R}$} & {$\left[\mathbb{Z}_{6}\right]_{R}$} & {$\left[\tilde{\mathbb{Z}}_{2}\right]_{R}$} & $\mathbb{Z}_{2}$ & {$\left[\tilde{\mathbb{Z}}_{4}\right]_{R}$} & {$\left[\mathbb{Z}_{2}\right]_{R}$} \\
\hline$Y_{0}^{c}$ & 4 & 0 & $-\frac{2}{3}$ & $-\frac{1}{3}$ & 0 & -1 & $\frac{1}{12}$ & $\frac{1}{3}$ & $\frac{1}{3}$ & $\frac{1}{2}$ & 0 & $\frac{3}{4}$ & 0 \\
\hline $\bar{Y}_{0}^{c}$ & 4 & 0 & $-\frac{2}{3}$ & $-\frac{1}{3}$ & 0 & 1 & $\frac{3}{4}$ & 0 & 0 & $\frac{1}{2}$ & 0 & $\frac{3}{4}$ & 0 \\
\hline$Y_{0}^{* c}$ & 4 & 0 & $-\frac{5}{3}$ & $-\frac{1}{3}$ & 0 & -1 & $\frac{3}{4}$ & 0 & 0 & $\frac{1}{2}$ & 0 & $\frac{3}{4}$ & 0 \\
\hline $\bar{Y}_{0}^{*} c$ & 4 & 0 & $-\frac{2}{3}$ & $\frac{2}{3}$ & 0 & -1 & $\frac{11}{12}$ & $\frac{2}{3}$ & $\frac{2}{3}$ & $\frac{1}{2}$ & 0 & $\frac{1}{4}$ & 0 \\
\hline$X_{1}^{c}$ & 4 & 1 & $-\frac{2}{3}$ & $-\frac{1}{3}$ & 0 & 0 & $\frac{5}{8}$ & $\frac{1}{3}$ & $\frac{1}{3}$ & 0 & 0 & 0 & 0 \\
\hline $\bar{X}_{1}^{c}$ & 4 & 1 & $-\frac{2}{3}$ & $-\frac{1}{3}$ & 0 & 0 & $\frac{1}{8}$ & 0 & $\frac{1}{3}$ & 0 & 0 & $\frac{1}{2}$ & 0 \\
\hline$Y_{1}^{c}$ & 4 & 1 & $-\frac{2}{3}$ & $-\frac{1}{3}$ & 0 & -1 & $\frac{17}{24}$ & 0 & $\frac{2}{3}$ & $\frac{1}{2}$ & 0 & $\frac{3}{4}$ & 0 \\
\hline $\bar{Y}_{1}^{c}$ & 4 & 1 & $-\frac{2}{3}$ & $-\frac{1}{3}$ & 0 & 1 & $\frac{1}{24}$ & $\frac{1}{3}$ & 0 & $\frac{1}{2}$ & 0 & $\frac{3}{4}$ & 0 \\
\hline$Y_{1}^{* c}$ & 4 & 1 & $-\frac{5}{3}$ & $-\frac{1}{3}$ & 0 & 1 & $\frac{17}{24}$ & 0 & $\frac{2}{3}$ & $\frac{1}{2}$ & 0 & $\frac{3}{4}$ & 0 \\
\hline $\bar{Y}_{1}^{* c}$ & 4 & 1 & $-\frac{2}{3}$ & $\frac{2}{3}$ & 0 & 1 & $\frac{7}{8}$ & $\frac{2}{3}$ & $\frac{1}{3}$ & $\frac{1}{2}$ & 0 & $\frac{1}{4}$ & 0 \\
\hline$X_{2}^{c}$ & 4 & 2 & $-\frac{2}{3}$ & $-\frac{1}{3}$ & 0 & -1 & $\frac{19}{24}$ & $\frac{1}{3}$ & $\frac{1}{3}$ & $\frac{1}{2}$ & 0 & $\frac{3}{4}$ & 0 \\
\hline $\bar{X}_{2}^{c}$ & 4 & 2 & $-\frac{2}{3}$ & $-\frac{1}{3}$ & 0 & 1 & $\frac{1}{8}$ & 0 & $\frac{2}{3}$ & $\frac{1}{2}$ & 0 & $\frac{3}{4}$ & 0 \\
\hline$Y_{2}^{c}$ & 4 & 2 & $-\frac{2}{3}$ & $-\frac{1}{3}$ & 0 & 0 & $\frac{1}{24}$ & 0 & $\frac{1}{3}$ & 0 & 0 & 0 & 0 \\
\hline $\bar{Y}_{2}^{c}$ & 4 & 2 & $-\frac{2}{3}$ & $-\frac{1}{3}$ & 0 & 0 & $\frac{7}{8}$ & $\frac{1}{3}$ & $\frac{2}{3}$ & 0 & 0 & $\frac{1}{2}$ & 0 \\
\hline$Y_{2}^{* c}$ & 4 & 2 & $-\frac{5}{3}$ & $-\frac{1}{3}$ & 0 & 0 & $\frac{13}{24}$ & 0 & $\frac{1}{3}$ & 0 & 0 & $\frac{1}{2}$ & 0 \\
\hline $\bar{Y}_{2}^{* c}$ & 4 & 2 & $-\frac{2}{3}$ & $\frac{2}{3}$ & 0 & 0 & $\frac{17}{24}$ & $\frac{2}{3}$ & 0 & 0 & 0 & 0 & 0 \\
\hline
\end{tabular}

Table 32: Discrete transformation phases $\eta_{\mathcal{S}}(s)$ for singlets $s$ at $\left(n_{2}, n_{2}^{\prime}\right)=(0,0)$. The tilde in $\tilde{\mathbb{Z}}_{N}$ denotes that the $N$-th application of the generator associated with the symmetry yields not necessarily an integer, but a phase which can be expressed by other generators. Note that the vacua $\mathcal{S}_{1}, \mathcal{S}_{2}$ additionally have an unbroken symmetry whose generator is proportional to $\frac{1}{2} t_{X}$, for $\mathcal{S}_{0}$ this holds up to $t_{5}$. $\mathcal{S}_{0}$ has $\mathrm{U}(1)_{5}$ as an unbroken continuous symmetry. 


\section{References}

[1] C. Amsler et al. [Particle Data Group], "Review of particle physics," Phys. Lett. B 667 (2008) 1.

[2] K. G. Wilson and J. B. Kogut, "The Renormalization group and the epsilon expansion," Phys. Rept. 12, 75 (1974).

[3] J. Kuti, L. Lin and Y. Shen, "Upper Bound On The Higgs Mass In The Standard Model," Phys. Rev. Lett. 61, 678 (1988);

M. Luscher and P. Weisz, "Is There A Strong Interaction Sector In The Standard Lattice Higgs Model?," Phys. Lett. B 212, 472 (1988).

[4] M. Niedermaier and M. Reuter, "The Asymptotic Safety Scenario in Quantum Gravity," Living Rev. Rel. 9, 5 (2006).

[5] H. Gies and M. M. Scherer, "Asymptotic safety of simple Yukawa systems", arXiv:0901.2459 [hep-th] (2009).

[6] G. Veneziano, "Construction of a crossing - symmetric, Regge behaved amplitude for linearly rising trajectories," Nuovo Cim. A 57, 190 (1968);

P. Ramond, "Dual Theory for Free Fermions," Phys. Rev. D 3 (1971) 2415;

A. Neveu and J. H. Schwarz, "Factorizable dual model of pions," Nucl. Phys. B 31, 86 (1971);

M. B. Green and J. H. Schwarz, "Supersymmetrical String Theories," Phys. Lett. B 109, 444 (1982).

[7] M. B. Green, J. H. Schwarz and E. Witten, Superstring Theory. Vol. 2: Loop Amplitudes, Anomalies And Phenomenology, Cambridge Monographs On Mathematical Physics (Cambridge University Press, Cambridge, 1546 UK, 1987), 596 p.

[8] J. Polchinski, String theory. Vol. 2: Superstring theory and beyond (Cambridge University Press, Cambridge, 1548 UK, 1998), 531 p.

[9] K. Becker, M. Becker and J. H. Schwarz, String theory and M-theory: A modern introduction, (Cambridge 1550 University Press, Cambridge, UK, 2007), 739 p.

[10] V. Braun, Y. H. He, B. A. Ovrut and T. Pantev, "A standard model from the E(8) x E(8) heterotic superstring," JHEP 0506, 039 (2005); [arXiv:hep-th/0502155];

V. Bouchard and R. Donagi, "An SU(5) heterotic standard model," Phys. Lett. B 633, 783 (2006); [arXiv:hep-th/0512149];

R. Blumenhagen, G. Honecker and T. Weigand, "Loop-corrected compactifications of the heterotic string with line bundles," JHEP 0506, 020 (2005); [arXiv:hepth/0504232]; 
R. Blumenhagen, S. Moster and T. Weigand, "Heterotic GUT and standard model vacua from simply connected Calabi-Yau manifolds," Nucl. Phys. B 751, 186 (2006); [arXiv:hep-th/0603015];

B. Andreas and G. Curio, "Deformations of Bundles and the Standard Model," Phys. Lett. B 655, 290 (2007); [arXiv:0706.1158 [hep-th]];

M. Kuriyama, H. Nakajima and T. Watari, A Theoretical Framework for R-parity Violation, arXiv:0802.2584 [hep-ph] (2008).

[11] T. Kobayashi, S. Raby and R. J. Zhang, "Constructing 5d orbifold grand unified theories from heterotic strings," Phys. Lett. B 593, 262 (2004); [arXiv:hep-ph/0403065];

T. Kobayashi, S. Raby and R. J. Zhang, "Searching for realistic 4d string models with a Pati-Salam symmetry: Orbifold grand unified theories from heterotic string compactification on a Z(6) orbifold," Nucl. Phys. B 704, 3 (2005). [arXiv:hep-ph/0409098].

[12] S. Forste, H. P. Nilles, P. K. S. Vaudrevange and A. Wingerter, "Heterotic brane world," Phys. Rev. D 70, 106008 (2004). [arXiv:hep-th/0406208].

[13] W. Buchmuller, K. Hamaguchi, O. Lebedev and M. Ratz, "Supersymmetric standard model from the heterotic string," Phys. Rev. Lett. 96, 121602 (2006); [arXiv:hep$\mathrm{ph} / 0511035]$;

W. Buchmuller, K. Hamaguchi, O. Lebedev and M. Ratz, "Supersymmetric standard model from the heterotic string. II," Nucl. Phys. B 785, 149 (2007). [arXiv:hepth/0606187].

[14] O. Lebedev, H. P. Nilles, S. Raby, S. Ramos-Sanchez, M. Ratz, P. K. S. Vaudrevange and A. Wingerter, "A mini-landscape of exact MSSM spectra in heterotic orbifolds," Phys. Lett. B 645, 88 (2007); [arXiv:hep-th/0611095];

O. Lebedev, H. P. Nilles, S. Ramos-Sanchez, M. Ratz and P. K. S. Vaudrevange, "Heterotic mini-landscape (II): completing the search for MSSM vacua in a $Z_{6}$ orbifold," Phys. Lett. B 668, 331 (2008). [arXiv:0807.4384 [hep-th]].

[15] J. E. Kim and B. Kyae, "String MSSM through flipped SU(5) from Z(12) orbifold", arXiv:hep-th/0608085 (2006);

J. E. Kim and B. Kyae, "Flipped SU(5) from Z(12-I) orbifold with Wilson line," Nucl. Phys. B 770, 47 (2007). [arXiv:hep-th/0608086].

[16] W. Buchmuller, C. Ludeling and J. Schmidt, "Local SU(5) Unification from the Heterotic String," JHEP 0709, 113 (2007). [arXiv:0707.1651 [hep-ph]].

[17] W. Buchmuller and J. Schmidt, "Higgs versus Matter in the Heterotic Landscape," Nucl. Phys. B 807, 265 (2009). [arXiv:0807.1046 [hep-th]]. 
[18] S. Ramos-Sanchez, Towards Low Energy Physics from the Heterotic String, arXiv:0812.3560 [hep-th] (2008);

P. K. S. Vaudrevange, Grand Unification in the Heterotic Brane World, arXiv:0812.3503 [hep-th] (2008).

[19] R. Blumenhagen, M. Cvetic, P. Langacker and G. Shiu, "Toward realistic intersecting D-brane models," Ann. Rev. Nucl. Part. Sci. 55, 71 (2005); [arXiv:hep-th/0502005];

F. Marchesano, "Progress in D-brane model building," Fortsch. Phys. 55, 491 (2007); [arXiv:hep-th/0702094];

R. Blumenhagen, B. Kors, D. Lust and S. Stieberger, "Four-dimensional String Compactifications with D-Branes, Orientifolds and Fluxes," Phys. Rept. 445, 1 (2007). [arXiv:hep-th/0610327].

[20] G. Honecker and T. Ott, "Getting just the supersymmetric standard model at intersecting branes on the Z(6)-orientifold," Phys. Rev. D 70, 126010 (2004) [Erratum-ibid. D 71, 069902 (2005)]; [arXiv:hep-th/0404055];

F. Gmeiner and G. Honecker, "Millions of Standard Models on Z6-prime?," JHEP 0807, 052 (2008). [arXiv:0806.3039 [hep-th]].

[21] R. Blumenhagen, V. Braun, T. W. Grimm and T. Weigand, "GUTs in Type IIB Orientifold Compactifications," Nucl. Phys. B 815, 1 (2009). [arXiv:0811.2936 [hepth]].

[22] K. R. Dienes and A. E. Faraggi, "Gauge coupling unification in realistic free fermionic string models," Nucl. Phys. B 457, 409 (1995). [arXiv:hep-th/9505046].

[23] R. Donagi and M. Wijnholt, "Model Building with F-Theory", arXiv:0802.2969 [hepth] (2008);

C. Beasley, J. J. Heckman and C. Vafa, "GUTs and Exceptional Branes in F-theory - I," JHEP 0901, 058 (2009); [arXiv:0802.3391 [hep-th]];

C. Beasley, J. J. Heckman and C. Vafa, "GUTs and Exceptional Branes in F-theory - II: Experimental Predictions," JHEP 0901, 059 (2009); [arXiv:0806.0102 [hep-th]];

R. Tatar and T. Watari, "GUT Relations from String Theory Compactifications," Nucl. Phys. B 810, 316 (2009); [arXiv:0806.0634 [hep-th]];

R. Blumenhagen, T. W. Grimm, B. Jurke and T. Weigand, "F-theory uplifts and GUTs", arXiv:0906.0013 [hep-th] (2009);

J. Marsano, N. Saulina and S. Schafer-Nameki, "Monodromies, Fluxes, and Compact Three-Generation F-theory GUTs," JHEP 0908, 046 (2009). [arXiv:0906.4672 [hepth]].

[24] H. Georgi and S. L. Glashow, "Unity Of All Elementary Particle Forces," Phys. Rev. Lett. 32, 438 (1974). 
[25] H. Georgi, in Particles and Fields 1974, edited by C. E. Carlson (AIP, New York, 1975) p. 575; H. Fritzsch and P. Minkowski, "Unified Interactions Of Leptons And Hadrons," Annals Phys. 93, 193 (1975).

[26] S. Raby, "SUSY GUT Model Building," Eur. Phys. J. C 59, 223 (2009). [arXiv:0807.4921 [hep-ph]].

[27] V. S. Kaplunovsky, "Mass Scales Of The String Unification," Phys. Rev. Lett. 55, 1036 (1985).

[28] E. Witten, "Symmetry Breaking Patterns In Superstring Models," Nucl. Phys. B 258, 75 (1985).

[29] D. J. Gross, J. A. Harvey, E. J. Martinec and R. Rohm, "The Heterotic String," Phys. Rev. Lett. 54, 502 (1985);

D. J. Gross, J. A. Harvey, E. J. Martinec and R. Rohm, "Heterotic String Theory. 1. The Free Heterotic String," Nucl. Phys. B 256, 253 (1985).

[30] L. J. Dixon, J. A. Harvey, C. Vafa and E. Witten, "Strings On Orbifolds," Nucl. Phys. B 261, 678 (1985);

L. J. Dixon, J. A. Harvey, C. Vafa and E. Witten, "Strings On Orbifolds. 2," Nucl. Phys. B 274, 285 (1986).

[31] Y. Kawamura, "Triplet-doublet splitting, proton stability and extra dimension," Prog. Theor. Phys. 105, 999 (2001); [arXiv:hep-ph/0012125];

G. Altarelli and F. Feruglio, "SU(5) grand unification in extra dimensions and proton decay," Phys. Lett. B 511, 257 (2001); [arXiv:hep-ph/0102301];

L. J. Hall and Y. Nomura, "Gauge unification in higher dimensions," Phys. Rev. D 64, 055003 (2001); [arXiv:hep-ph/0103125];

A. Hebecker and J. March-Russell, "A minimal S(1)/(Z(2) x Z'(2)) orbifold GUT," Nucl. Phys. B 613, 3 (2001); [arXiv:hep-ph/0106166];

R. Dermisek and A. Mafi, "SO(10) grand unification in five dimensions: Proton decay and the mu problem," Phys. Rev. D 65, 055002 (2002); [arXiv:hep-ph/0108139];

L. J. Hall and Y. Nomura, "A complete theory of grand unification in five dimensions," Phys. Rev. D 66, 075004 (2002); [arXiv:hep-ph/0205067];

H. D. Kim and S. Raby, "Unification in 5D SO(10)," JHEP 0301, 056 (2003). [arXiv:hep-ph/0212348].

[32] L. J. Hall, H. Murayama and Y. Nomura, "Wilson lines and symmetry breaking on orbifolds," Nucl. Phys. B 645, 85 (2002). [arXiv:hep-th/0107245].

[33] L. J. Hall, Y. Nomura, T. Okui and D. Tucker-Smith, "SO(10) unified theories in six dimensions," Phys. Rev. D 65, 035008 (2002). [arXiv:hep-ph/0108071]. 
[34] T. Asaka, W. Buchmuller and L. Covi, "Gauge unification in six dimensions," Phys. Lett. B 523, 199 (2001); [arXiv:hep-ph/0108021];

T. Asaka, W. Buchmuller and L. Covi, "Exceptional coset spaces and unification in six dimensions," Phys. Lett. B 540, 295 (2002); [arXiv:hep-ph/0204358];

T. Asaka, W. Buchmuller and L. Covi, "Quarks and leptons between branes and bulk," Phys. Lett. B 563, 209 (2003). [arXiv:hep-ph/0304142].

[35] T. Asaka, W. Buchmuller and L. Covi, "Bulk and brane anomalies in six dimensions," Nucl. Phys. B 648, 231 (2003). [arXiv:hep-ph/0209144].

[36] A. Hebecker, J. March-Russell and R. Ziegler, "Inducing the $\mu$ and the $B \mu$ Term by the Radion and the 5d Chern-Simons Term," JHEP 0908, 064 (2009); [arXiv:0801.4101 [hep-ph]];

C. Gross and A. Hebecker, "A Realistic Unified Gauge Coupling from the MicroLandscape of Orbifold GUTs," Nucl. Phys. B 821, 354 (2009); [arXiv:0812.4267 [hep$\mathrm{ph}]$;

F. Brummer, S. Fichet, A. Hebecker and S. Kraml, "Phenomenology of Supersymmetric Gauge-Higgs Unification", arXiv:0906.2957 [hep-ph] (2009).

[37] W. Buchmuller, J. Moller and J. Schmidt, "Light Moduli in Almost No-Scale Models," arXiv:0909.0482 [hep-ph].

[38] W. Buchmuller, K. Hamaguchi, O. Lebedev and M. Ratz, "Dual models of gauge unification in various dimensions," Nucl. Phys. B 712, 139 (2005); [arXiv:hep-ph/0412318];

W. Buchmuller, K. Hamaguchi, O. Lebedev and M. Ratz, "Local grand unification", arXiv:hep-ph/0512326 (2005).

[39] L. E. Ibanez, H. P. Nilles and F. Quevedo, "Orbifolds And Wilson Lines," Phys. Lett. B 187, 25 (1987);

L. E. Ibanez, J. E. Kim, H. P. Nilles and F. Quevedo, "Orbifold Compactifications With Three Families Of SU(3) X SU(2) X U(1)**N," Phys. Lett. B 191, 282 (1987);

J. A. Casas and C. Munoz, "Three Generation SU(3) x SU(2) x U(1)-Y x U(1) Orbifold Models Through Fayet-Iliopoulos Terms," Phys. Lett. B 209, 214 (1988);

J. A. Casas and C. Munoz, "Three Generation SU(3) x SU(2) x U(1)-Y Models from Orbifolds," Phys. Lett. B 214, 63 (1988);

A. Font, L. E. Ibanez, H. P. Nilles and F. Quevedo, "Yukawa Couplings In Degenerate Orbifolds: Towards A Realistic SU(3) X SU(2) X U(1) Superstring," Phys. Lett. 210B, 101 (1988) [Erratum-ibid. B 213, 564 (1988)];

J. A. Casas, E. K. Katehou and C. Munoz, "U(1) Charges in Orbifolds: Anomaly Cancellation and Phenomenological Consequences," Nucl. Phys. B 317, 171 (1989); 
J. A. Casas, M. Mondragon and C. Munoz, "Reducing the number of candidates to standard model in the Z(3) orbifold", Phys. Lett. B 230, 63 (1989);

A. Font, L. E. Ibanez, F. Quevedo and A. Sierra, "The Construction of 'Realistic' Four-Dimensional Strings Through Orbifolds," Nucl. Phys. B 331, 421 (1990).

[40] A. Font, L. E. Ibanez, H. P. Nilles and F. Quevedo, "Degenerate Orbifolds," Nucl. Phys. B 307, 109 (1988) [Erratum-ibid. B 310, 764 (1988)].

[41] L. E. Ibanez, J. Mas, H. P. Nilles and F. Quevedo, "Heterotic Strings In Symmetric And Asymmetric Orbifold Backgrounds," Nucl. Phys. B 301, 157 (1988).

[42] E. Witten, "Strong Coupling Expansion Of Calabi-Yau Compactification," Nucl. Phys. B 471, 135 (1996); [arXiv:hep-th/9602070];

T. Banks and M. Dine, "Couplings and Scales in Strongly Coupled Heterotic String Theory," Nucl. Phys. B 479, 173 (1996). [arXiv:hep-th/9605136].

[43] A. Hebecker and M. Trapletti, "Gauge unification in highly anisotropic string compactifications," Nucl. Phys. B 713, 173 (2005). [arXiv:hep-th/0411131].

[44] B. Dundee, S. Raby and A. Wingerter, "Reconciling Grand Unification with Strings by Anisotropic Compactifications," Phys. Rev. D 78, 066006 (2008); [arXiv:0805.4186 [hep-th]];

B. Dundee, S. Raby and A. Wingerter, "Addendum to Reconciling Grand Unification with Strings by Anisotropic Compactifications", arXiv:0811.4026 [hep-th] (2008);

B. Dundee and S. Raby, "On the string coupling in a class of stringy orbifold GUTs", arXiv:0808.0992 [hep-th] (2008).

[45] W. Buchmuller, R. Catena and K. Schmidt-Hoberg, "Small Extra Dimensions from the Interplay of Gauge and Supersymmetry Breaking," Nucl. Phys. B 804 (2008) 70; [arXiv:0803.4501 [hep-ph]];

W. Buchmuller, R. Catena and K. Schmidt-Hoberg, "Enhanced Symmetries of Orbifolds from Moduli Stabilization," Nucl. Phys. B 821, 1 (2009). [arXiv:0902.4512 [hepth]].

[46] J. J. Atick, L. J. Dixon and A. Sen, "String Calculation Of Fayet-Iliopoulos D Terms In Arbitrary Supersymmetric Compactifications," Nucl. Phys. B 292, 109 (1987).

[47] G. Cleaver, M. Cvetic, J. R. Espinosa, L. L. Everett and P. Langacker, "Classification of flat directions in perturbative heterotic superstring vacua with anomalous U(1)," Nucl. Phys. B 525, 3 (1998). [arXiv:hep-th/9711178].

[48] C. D. Froggatt and H. B. Nielsen, "Hierarchy Of Quark Masses, Cabibbo Angles And CP Violation," Nucl. Phys. B 147, 277 (1979). 
[49] O. Lebedev, H. P. Nilles, S. Raby, S. Ramos-Sanchez, M. Ratz, P. K. S. Vaudrevange and A. Wingerter, "The Heterotic Road to the MSSM with R parity," Phys. Rev. D 77, 046013 (2008). [arXiv:0708.2691 [hep-th]].

[50] R. Kappl, H. P. Nilles, S. Ramos-Sanchez, M. Ratz, K. Schmidt-Hoberg and P. K. S. Vaudrevange, "Large hierarchies from approximate R symmetries," Phys. Rev. Lett. 102, 121602 (2009). [arXiv:0812.2120 [hep-th]].

[51] P. Candelas, G. T. Horowitz, A. Strominger and E. Witten, "Vacuum Configurations For Superstrings," Nucl. Phys. B 258, 46 (1985).

[52] P. S. Aspinwall, "Resolution of orbifold singularities in string theory", arXiv:hepth/9403123 (1994).

[53] G. Honecker and M. Trapletti, "Merging heterotic orbifolds and K3 compactifications with line bundles," JHEP 0701, 051 (2007). [arXiv:hep-th/0612030].

[54] S. G. Nibbelink, M. Trapletti and M. Walter, "Resolutions of $C^{n} / Z_{n}$ Orbifolds, their U(1) Bundles, and Applications to String Model Building," JHEP 0703, 035 (2007). [arXiv:hep-th/0701227].

[55] D. Lust, S. Reffert, E. Scheidegger and S. Stieberger, "Resolved toroidal orbifolds and their orientifolds," Adv. Theor. Math. Phys. 12, 67 (2008). [arXiv:hep-th/0609014].

[56] S. G. Nibbelink, J. Held, F. Ruehle, M. Trapletti and P. K. S. Vaudrevange, "Heterotic Z6-II MSSM Orbifolds in Blowup," JHEP 0903, 005 (2009). [arXiv:0901.3059 [hepth]].

[57] D. Bailin and A. Love, "Orbifold compactifications of string theory," Phys. Rept. 315, 285 (1999);

J. T. Giedt, "Heterotic orbifolds", arXiv:hep-ph/0204315 (2002);

M. K. Gaillard and B. D. Nelson, "Kaehler stabilized, modular invariant heterotic string models," Int. J. Mod. Phys. A 22, 1451 (2007). [arXiv:hep-th/0703227].

[58] Y. Katsuki, Y. Kawamura, T. Kobayashi, N. Ohtsubo, Y. Ono and K. Tanioka, "Z(N) Orbifold Models," Nucl. Phys. B 341, 611 (1990).

[59] C. Vafa, "Modular Invariance And Discrete Torsion On Orbifolds," Nucl. Phys. B 273, 592 (1986).

[60] F. Gliozzi, J. Scherk and D. I. Olive, "Supergravity And The Spinor Dual Model," Phys. Lett. B 65, 282 (1976);

F. Gliozzi, J. Scherk and D. I. Olive, "Supersymmetry, Supergravity Theories And The Dual Spinor Model," Nucl. Phys. B 122, 253 (1977). 
[61] L. J. Dixon, D. Friedan, E. J. Martinec and S. H. Shenker, "The Conformal Field Theory Of Orbifolds," Nucl. Phys. B 282, 13 (1987);

S. Hamidi and C. Vafa, "Interactions on Orbifolds," Nucl. Phys. B 279, 465 (1987);

J. Erler, D. Jungnickel, M. Spalinski and S. Stieberger, "Higher twisted sector couplings of Z(N) orbifolds," Nucl. Phys. B 397, 379 (1993); [arXiv:hep-th/9207049];

J. A. Casas, F. Gomez and C. Munoz, "Complete structure of Z(n) Yukawa couplings," Int. J. Mod. Phys. A 8, 455 (1993); [arXiv:hep-th/9110060];

K. S. Choi and T. Kobayashi, "Higher Order Couplings from Heterotic Orbifold Theory," Nucl. Phys. B 797, 295 (2008). [arXiv:0711.4894 [hep-th]].

[62] S. Forste, H. P. Nilles and A. Wingerter, "The Higgs mechanism in heterotic orbifolds," Phys. Rev. D 73, 066011 (2006); [arXiv:hep-th/0512270];

O. Lebedev, H. P. Nilles, S. Raby, S. Ramos-Sanchez, M. Ratz, P. K. S. Vaudrevange and A. Wingerter, "Low Energy Supersymmetry from the Heterotic Landscape," Phys. Rev. Lett. 98, 181602 (2007); [arXiv:hep-th/0611203];

W. Buchmuller, K. Hamaguchi, O. Lebedev, S. Ramos-Sanchez and M. Ratz, "Seesaw neutrinos from the heterotic string," Phys. Rev. Lett. 99, 021601 (2007). [arXiv:hep$\mathrm{ph} / 0703078]$.

K. S. Choi, H. P. Nilles, S. Ramos-Sanchez and P. K. S. Vaudrevange, "Accions," Phys. Lett. B 675, 381 (2009); [arXiv:0902.3070 [hep-th]];

P. Hosteins, R. Kappl, M. Ratz and K. Schmidt-Hoberg, "Gauge-top unification," JHEP 0907, 029 (2009). [arXiv:0905.3323 [hep-ph]].

[63] H. Nishino and E. Sezgin, "The Complete $N=2, D=6$ Supergravity With Matter And Yang-Mills Couplings," Nucl. Phys. B 278, 353 (1986).

[64] N. Arkani-Hamed, T. Gregoire and J. G. Wacker, "Higher dimensional supersymmetry in 4D superspace," JHEP 0203, 055 (2002). [arXiv:hep-th/0101233].

[65] M. B. Green and J. H. Schwarz, "Anomaly Cancellation In Supersymmetric D=10 Gauge Theory And Superstring Theory," Phys. Lett. B 149, 117 (1984).

[66] C. A. Scrucca and M. Serone, "Anomalies in field theories with extra dimensions," Int. J. Mod. Phys. A 19, 2579 (2004). [arXiv:hep-th/0403163].

[67] S. D. Avramis, "Anomaly-free supergravities in six dimensions", arXiv:hepth/0611133 (2006).

[68] S. L. Adler, "Axial vector vertex in spinor electrodynamics," Phys. Rev. 177, 2426 (1969);

J. S. Bell and R. Jackiw, "A PCAC puzzle: pi0 $\rightarrow$ gamma gamma in the sigma model," Nuovo Cim. A 60, 47 (1969). 
[69] J. Wess and B. Zumino, "Consequences of anomalous Ward identities," Phys. Lett. B 37, 95 (1971).

[70] G. 't Hooft, "Symmetry breaking through Bell-Jackiw anomalies," Phys. Rev. Lett. 37, 8 (1976);

G. 't Hooft, "Computation of the quantum effects due to a four-dimensional pseudoparticle," Phys. Rev. D 14, 3432 (1976) [Erratum-ibid. D 18, 2199 (1978)].

[71] K. Fujikawa, "Path Integral Measure For Gauge Invariant Fermion Theories," Phys. Rev. Lett. 42, 1195 (1979);

K. Fujikawa, "Path Integral For Gauge Theories With Fermions," Phys. Rev. D 21, 2848 (1980) [Erratum-ibid. D 22, 1499 (1980)].

[72] L. Alvarez-Gaume and E. Witten, "Gravitational Anomalies," Nucl. Phys. B 234, 269 (1984).

[73] R. Stora, Algebraic Structure And Topological Origin Of Anomalies, in Progress in Gauge Field Theory, edited by G. 't Hooft et al. (Plenum Press, New York, 1984), pp 373-401;

B. Zumino, Chiral Anomalies And Differential Geometry, in Relativity, groups and topology II, edited by B. DeWitt and R. Stora (Elsevier, Amsterdam, 1984).

[74] L. Alvarez-Gaume and P. H. Ginsparg, "The Topological Meaning Of Nonabelian Anomalies," Nucl. Phys. B 243, 449 (1984).

[75] G. von Gersdorff and M. Quiros, "Localized anomalies in orbifold gauge theories," Phys. Rev. D 68, 105002 (2003); [arXiv:hep-th/0305024];

G. von Gersdorff, "Anomalies on six dimensional orbifolds," JHEP 0703, 083 (2007). [arXiv:hep-th/0612212].

[76] M. B. Green, J. H. Schwarz and P. C. West, "Anomaly Free Chiral Theories In SixDimensions," Nucl. Phys. B 254, 327 (1985).

[77] J. Erler, "Anomaly Cancellation In Six-Dimensions," J. Math. Phys. 35, 1819 (1994). [arXiv:hep-th/9304104].

[78] A. Hebecker and J. March-Russell, "The structure of GUT breaking by orbifolding," Nucl. Phys. B 625, 128 (2002). [arXiv:hep-ph/0107039].

[79] H. M. Lee, H. P. Nilles and M. Zucker, "Spontaneous localization of bulk fields: The six-dimensional case," Nucl. Phys. B 680, 177 (2004). [arXiv:hep-th/0309195].

[80] J. C. Pati and A. Salam, "Unified Lepton-Hadron Symmetry And A Gauge Theory Of The Basic Interactions," Phys. Rev. D 8, 1240 (1973). 
[81] S. Dimopoulos, S. Raby and F. Wilczek, "Proton Decay In Supersymmetric Models," Phys. Lett. B 112, 133 (1982).

[82] S. Groot Nibbelink, H. P. Nilles and M. Olechowski, "Spontaneous localization of bulk matter fields," Phys. Lett. B 536, 270 (2002). [arXiv:hep-th/0203055].

[83] P. S. Aspinwall, "K3 surfaces and string duality", arXiv:hep-th/9611137 (1996).

[84] M. Bershadsky, K. A. Intriligator, S. Kachru, D. R. Morrison, V. Sadov and C. Vafa, "Geometric singularities and enhanced gauge symmetries," Nucl. Phys. B 481, 215 (1996). [arXiv:hep-th/9605200].

[85] S. Raby, Private communication.

[86] C. Borcea, K3 surfaces with involution and mirror pairs of Calabi-Yau manifolds, in Mirror symmetry II, edited by B. Greene and S. T. Yau (American Mathematical Society/International Press, Providence, USA, 1997), pp 717-743. 


\section{Index}

All-order superpotential, 94

Anisotropic geometry, 35

Anomaly, 56, 97

chiral, 59

consistent, 59

irreducible, 61

polynomial

bulk, 60, 63, 66, 111

local, 63, 67, 112

reducible, 61, 66, 67

Antisymmetric tensor field, 41, 65

Boundary conditions

bosons, 12

equivalent, 17

fermions, 13

gauge fields, 15

Cartan generators, 24, 42, 108

Cartan matrix, 43, 46, 109

Cartan-Weyl labels, 46, 110

Center-of-mass momentum, 18

Charge kernel, 86, 88, 91

Chern character, 60

Chirality, see Weyl fermion

Compact space, 8, 10

Complex coordinates, 10, 11

Conjugacy class, 11, 26, 30

Consistency conditions, see Orbifold projection conditions

Continous symmetry, 85 charge vector $\mathbf{Q}, 79,85$

Couplings, 25, 32, 77, 81, 94

Coxeter element, 105, 107

$D$-terms, 73

Decoupling, 74, 76

Dirac genus, 60

Discrete symmetry, 34, 85

charge vector $\mathcal{K}, 79,85$ transformation phase, 122

Dual Coxeter number, 66

Dynkin labels, 46, 110

Effective orbifold, 35

field theory, 53

geometry, 37

projection conditions, 36

Equations of motion, 9 solutions, 8,15

Euler characteristic, 97

Exotics, 53, 73, 75, 76, 88, 91

$F$-terms, 74, 96

False vacuum, 68

Fayet-Iliopoulos term, 74

Field strength two-form, 111

Field-strength two-form, 59

Fixed point/plane/torus, 12 solutions, 103

Gamma-phases, 20, 21, 30, 36, 38, 39, 107

Gauge bundle, 97

Gauge embedding, 14, 32

Gauge symmetry

bulk, 43, 98

charges, 15

four dimensions, 45

K3, 98

local, 45

Gauge-Higgs unification, 55, 86

Geometrical moduli, 24, 41, 99

Geometrical phase, see Space group transformation phase

Gravitino, 24, 61

Green-Schwarz mechanism, 65, 66

GSO projection, 16

Helicity, 23, 24

Hidden brane, 52, 115 
Higgs candidates, 76, 81

Hilbert space, 16 operators, 17 states, see Sector states

Instanton number, 97

K3 surface, 97

Lattice

translations, 11

vectors, 11

order, 14, 20

Left-movers, 8

Lie group/algebra, 108

Little group, 10, 14, 40 charges, 14

Local parities, 55

Localization of states, 18

Mass equations, 22

Matter parity, 70, 89, 92

Maximal vacuum, 86, 88, 91

Minimal vacuum, 77

Modular invariance conditions, 15

Moduli space

gauge, 98

geometrical, 97

$\mu$-term, 81, 87, 90

Multiplet

hypermultiplet (six dimensions), 41

supergravity

four dimensions, 24

six dimensions, 41

ten dimensions, 23

tensor (six dimensions), 41

vector

four dimensions, 44

six dimensions, 42

ten dimensions, 24

Orbifold

geometry, 10, 11, 27

projection conditions, 18, 21, 36, 39, 54
Oscillator numbers, 17, 19

Partial gauge-Higgs unification, 55, 90

Phenomenological requirements, 84

Physical states, see Orbifold projection conditions

Physical vacuum, 68, 76, 79

Point group, 10, 23

Proton decay dimension-five, 82, 88, 89, 92, 124

dimension-four, 70, 82

$R$-symmetry, 19, 25, 82

Riemann curvature two-form, 59

Right-movers, 8, 13

Roots, 42, 108

Sector, 16, 29, 38

gravity, 22,40

states, $17-20$

twisted, 16, 35, 48

untwisted, 16, 22, 47

Shift vector, 14

local, 18, 32

Simple roots, 42, 45, 109

Space group, 11

eigenstate, 19, 20, 30, 107

invariance, see Orbifold

projection conditions

transformation

coordinates, 11, 19, 30

fermions, 13

gauge fields, 14

operators, 19

phase, 19-21, 31, 39, 107

states, 19

Spectrum

bulk, 48, 50, 98

exotic fixed points, 53, 54, 118, 123-125

GUT fixed points, 52, 54-57

K3, 98

zero modes, 122

Standard model families, 51, 69, 71, 73, 76

Stora-Zumino descent relations, 59 
String selection rules, 25

space group, 26, 34, 103

SU(5) GUT, 68

Sub-lattice, 11, 33

Sub-twists, 28, 37

Superposition, see Space group eigenstate

Tadpole cancelation on K3, 97

Trace identities, 61

Twist vector, 10

condition for $\mathcal{N}=1,24$

local, 18

Two Higgs pairs, 88

$\mathrm{U}(1)$

anomalous, 64, 114

$B-L, 70,73$

canonical normalization, 62

charges, 47

factors, 43, 118

generators, 44

$X, 70,73,82,89,92$

Unbroken symmetries, 79, 89, 92, 122

Universal mass term, 75

Weight lattice of $\mathrm{SO}(8), 14$

Weights, 108

Weyl fermion

four dimensions, 23

six dimensions, 41, 61

Wilson lines, 14, 32, 38

Winding modes/numbers, 18, 103

World-sheet

action, 8

coordinates, 8

fields, 8

Yukawa couplings

four dimensions, 83, 93

six dimensions, 82, 89, 93

$\mathbb{Z}_{6 \text {-II }}$ orbifold, 27

Fixed points/tori, 29

gauge embedding, 32

quantum numbers $\gamma^{\prime}, 31,108$ 